\title{
The Effects of Unsteady Loads on Human Postural Stability while Exposed to Shipboard Motions
}

by

\author{
Heather Morris
}

\author{
A Thesis submitted to \\ the Faculty of Graduate Studies and Postdoctoral Affairs \\ in partial fulfilment of \\ the requirements for the degree of \\ Master of Applied Science \\ in
}
Mechanical and Aerospace Engineering
Carleton University
Ottawa, Ontario, Canada
January 2013

Copyright (C)

2013 - Heather Morris 
Library and Archives

Canada

Published Heritage

Branch

395 Wellington Street

Ottawa ON K1A ON4

Canada
Bibliothèque et

Archives Canada

Direction du

Patrimoine de l'édition

395 , rue Wellington

Ottawa ON K1A ON4

Canada
Your file Votre référence

ISBN: 978-0-494-94639-8

Our file Notre référence

ISBN: $978-0-494-94639-8$
NOTICE:

The author has granted a nonexclusive license allowing Library and Archives Canada to reproduce, publish, archive, preserve, conserve, communicate to the public by telecommunication or on the Internet, loan, distrbute and sell theses worldwide, for commercial or noncommercial purposes, in microform, paper, electronic and/or any other formats.

The author retains copyright ownership and moral rights in this thesis. Neither the thesis nor substantial extracts from it may be printed or otherwise reproduced without the author's permission.
AVIS:

L'auteur a accordé une licence non exclusive permettant à la Bibliothèque et Archives Canada de reproduire, publier, archiver, sauvegarder, conserver, transmettre au public par télécommunication ou par l'Internet, prêter, distribuer et vendre des thèses partout dans le monde, à des fins commerciales ou autres, sur support microforme, papier, électronique et/ou autres formats.

L'auteur conserve la propriété du droit d'auteur et des droits moraux qui protege cette thèse. $\mathrm{Ni}$ la thèse ni des extraits substantiels de celle-ci ne doivent être imprimés ou autrement reproduits sans son autorisation.
In compliance with the Canadian Privacy Act some supporting forms may have been removed from this thesis.

While these forms may be included in the document page count, their removal does not represent any loss of content from the thesis.
Conformément à la loi canadienne sur la protection de la vie privée, quelques formulaires secondaires ont été enlevés de cette thèse.

Bien que ces formulaires aient inclus dans la pagination, il n'y aura aucun contenu manquant. 


\section{Abstract}

A collaborative project was undertaken between Defence Research and Development Canada and Carleton University to investigate the issue of postural stability in shipboard environments. The author's contribution to the project comprised of the generalization of the rigid body Graham model with time-varying unsteady loads, development of a two-degree of freedom unsteady in-plane cart load model, implementation of all models in the distributed Virtual Flight Deck-Real Time (VFD-RT) simulation environment, and validation of the developed models through physical experimentation. Also, the motion induced interruption (MII) model was generalized and validated through experiments on the Stewart platform and at sea on CFAV Quest. The results show that the unsteady loads have a negative effect on postural stability as the number of MII events increase. As well, a relationship was found between ship heading and optimum stance angle which can be used to determine stance to reduce MII events. 


\section{Acknowledgments}

I would like to thank the following people who helped me along with the PSM project and the MII experiments.

I would like to thank Nick Bourgois for his help in the instrumentation and data collection for the full-scale apparatus. His help and guidance with the VFD-RT software was instrumental to my success; and also, for the software controller for the six-degree-of-freedom motion base. Thanks to Jamie Laveille as well for the fantastic design and construction of the physical apparatus for the experimentation.

To Angelo Rajendram for the construction of the physical MII man as well as developing the Arduino-based data collection system for the physical experiments.

I would like to thank Eric Thornhill from DRDC Atlantic for allowing the PSM project to be on board Quest for the high seas trial. As well, a thanks to the support staff from DRDC for helping assemble the apparatus and making sure everything was taken care of on board. Also, thank you to the crew on board for taking care of us while we performed our experiments. As well, to Kevin McTaggart and Jim Colwell also from DRDC Atlantic for the funding for this project.

Finally, thank you to Rob Langlois for your patience and guidance. I could not have asked for anyone better to guide me through this process. 


\section{Table of Contents}

$\begin{array}{ll}\text { Abstract } & \text { ii }\end{array}$

$\begin{array}{ll}\text { Acknowledgments } & \text { iii }\end{array}$

Table of Contents $\quad$ iv

List of Tables $\quad$ vii

List of Figures $\quad$ ix

List of Symbols $\quad$ XV

1 Introduction 1

1.1 Motivation ....................... 1

1.2 Literature Review . . . . . . . . . . . . . . . . 3

1.2.1 Physiology of Human Postural Stability . . . . . . . . . . . 3

1.2.2 Postural Stability Modelling . . . . . . . . . . . 5 5

1.2.3 Motion Induced Interruptions . . . . . . . . . . . . 16

1.3 Overview. ........................... 24

2 Model Development $\quad 26$

2.1 Three-dimensional Graham Model . . . . . . . . . . . . . . . 26

2.1.1 Kinematics of the Graham Model . . . . . . . . . . . . . . 27 
2.1.2 Dynamics of the Graham Model . . . . . . . . . . . 29

2.2 Unsteady Cart Load Model _ . . . . . . . . . . . . . . . . . 32

2.2.1 Model Definition and Coordinate Systems . . . . . . . 32

2.2.2 Kinematics for Two-body Cart . . . . . . . . . . 34

2.2 .3 Dynamics for Two-body Cart . . . . . . . . . . . 39

2.2 .4 Interface Force $\ldots \ldots \ldots \ldots$

2.3 Stand-alone Computer Implementation . . . . . . . . . . . . . 52

2.4 VFD-RT Simulation Implementation $\ldots \ldots \ldots \ldots$

2.5 Computer Simulation Verification . . . . . . . . . . . . 59

3 Model Validation $\quad 63$

3.1 Overview. . . . . . . . . . . . . . . . . . 63

3.2 Validation of the Graham Stability Model with the Locked Inverted Pendulum . . . . . . . . . . . . . . . . . . 64

3.3 Computational Validation of the Cart Load Model . . . . . . . . 66

3.4 Validation of the Graham Model Through Experimentation . . . . . 76

3.4.1 Experimental Apparatus . . . . . . . . . . . . . 76

3.4.2 Experimental Validation of the Graham Stability Model ... 84

3.4.3 Validation of the Coupled Graham Model with the Pendulum and Cart Loads through Experimentation $\ldots \ldots \ldots$

4 MII Analysis $\quad 99$

4.1 MII Definition and Concept . . . . . . . . . . . . . . . . . 99

4.2 MII Validation Experiments . . . . . . . . . . . . . . . . 102

4.2 .1 Physical Apparatus Design . . . . . . . . . . . . . . 102

4.2.2 MII Detection with Stance Geometry Validation Experiments 104

4.2.3 Comparison of Results from the MII Stance Experiments . . . 107 


\subsubsection{Validation Against Footprint Model Experimentation on CFAV}

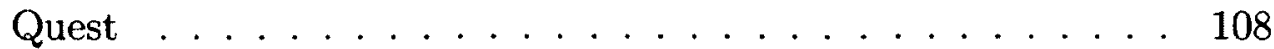

4.3 Effect of Stance Geometry . . . . . . . . . . . . . . . . 115

4.4 Effect of Unsteady Dynamic Loads . . . . . . . . . . . . . . 123

5 Conclusion $\quad 129$

5.1 Discussion of Results . . . . . . . . . . . . . . . . . . . 129

5.2 Conclusion . . . . . . . . . . . . . . . . 133

5.3 Future Work . . . . . . . . . . . . . . . . . . . . . . . . . . 134

$\begin{array}{ll}\text { List of References } & 136\end{array}$ 


\section{List of Tables}

2 NATO Sea State Numerical Table for the Open Ocean North Atlantic 53

3 Simulation parameters used for validating GRM3D against PSM3D . 65

4 Simulation parameters used for CRT3D validation . . . . . . . . . 70

5 Results from translational forced frequency excitation on the cart for the case where $\zeta=0.35 \ldots \ldots \ldots \ldots \ldots \ldots 71$

6 Results from translational forced frequency excitation on the cart for the case where $\zeta=0.5 \ldots \ldots \ldots \ldots \ldots \ldots$

7 Results from translational forced frequency excitation on the cart for the case where $\zeta=0.25 \ldots \ldots \ldots \ldots \ldots$

8 Results from rotational forced frequency excitation on the cart for the

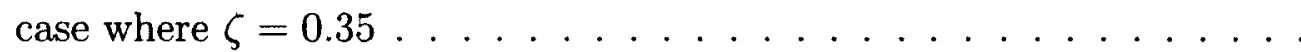

9 Results from rotational forced frequency excitation on the cart for the case where $\zeta=0.5 \ldots \ldots \ldots \ldots \ldots \ldots \ldots \ldots$

10 Results from rotational forced frequency excitation on the cart for the

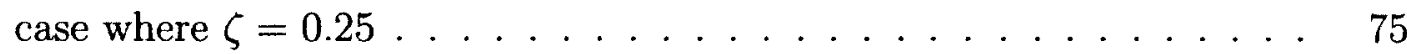

11 Simulation parameters used with GRM3D and PSM3D . . . . . 84

12 MII occurrence times for the straight stance at 0 degrees offset obtained from the experimental apparatus and simulation . . . . . . . 108

13 MII occurrence times for the staggered stance at 0 degrees offset obtained from the experimental apparatus and simulation . . . . . . 109 
14 MII occurrence times for the straight stance at 90 degrees in beam seas obtained from the experimental apparatus on Quest experiments and simulation . . . . . . . . . . . . . . . . . 110

15 MII occurrence times for the staggered stance at 0 degrees in beam seas obtained from the experimental apparatus on Quest experiments

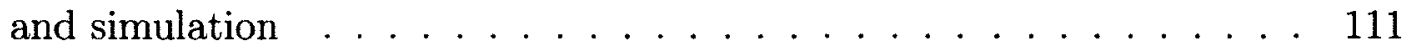

16 Simulation parameters used for CRT3D load investigation . . . . . 124

17 Simulation parameters used for PND3D load investigation . . . . . 125

18 Simulation results for number of MIIs occurring at sea state $4 \ldots 125$

19 Simulation results for number of MIIs occurring at sea state $5 \ldots 126$

20 Simulation results for number of MIIs occurring at sea state $6 \ldots 127$ 


\section{List of Figures}

1 Crew member handling a wave buoy suspended by a crane. . . . . . . 2

2 Feedback postural stability model. . . . . . . . . . . . . . 4

3 Golliday et al. two-link inverted pendulum model . . . . . . . . . 7

4 Hemami et al. three-link inverted pendulum model . . . . . . . . . . 8

5 Koozekanani et al. four-bar linkage model . . . . . . . . . . . . . 9

6 Hemami et al. two-link inverted pendulum model with interface forces 11

$7 \quad$ Barin multilink inverted pendulum model . . . . . . . . . . . . . . . . 12

8 Iqbal et al. frontal postural stability model . . . . . . . . . . . . . . . 14

9 Hemami et al. muscle model for three-link pendulum . . . . . . . . 15

10 Moments on the Graham MII Model . . . . . . . . . . . . . 18

11 Frontal and sagittal plane models for McKee and Langlois's articulated postural model ...................... . . 21

12 Coordinate systems used in the derivation of the three-dimensional Graham shipboard postural stability model . . . . . . . . . . . 27

13 Forces acting on the three-dimensional Graham model. . . . . . . . . 29

14 Coordinate systems used in the derivation of in-plane cart load model 33

15 Free body diagram of the two cart masses with the state variables . . 39

16 Schematic representation of postural stability models interacting with the pendulating load (top) and cart load (bottom) . . . . . . . . 51

17 Directed element model. . . . . . . . . . . . . . . . . 51 
18 Visualization of DEPSM simulation environment and solution flow .

19 Connection and data transfer comparison between the VFD-RT and HLA architectures ................. . . 57

20 Connection and data transfer between VFD-RT executables . . . . 58

21 Longitudinal force $\left(F_{x}\right)$ computed using GRM3D implemented in the VFD-RT and the DEPSM simulation environments . . . . . . . 60

22 Lateral force $\left(F_{y}\right)$ computed using GRM3D implemented in the VFDRT and the DEPSM simulation environments . . . . . . . . . . 60

23 Vertical force $\left(F_{z}\right)$ computed using GRM3D implemented in the VFDRT and the DEPSM simulation environments . . . . . . . . . 61

24 Longitudinal moment $\left(M_{x}\right)$ computed using GRM3D implemented in the VFD-RT and the DEPSM simulation environments . . . . . . . 61

25 Lateral moment $\left(M_{y}\right)$ computed using GRM3D implemented in the VFD-RT and the DEPSM simulation environments . . . . . . . . . 62

26 Vertical moment $\left(M_{z}\right)$ computed using GRM3D implemented in the VFD-RT and the DEPSM simulation environments . . . . . . . 62

27 Longitudinal force $\left(F_{x}\right)$ for typical ship motion computed using GRM3D and PSM3D . . . . . . . . . . . . . . 67

28 Lateral force $\left(F_{y}\right)$ for typical ship motion computed using GRM3D and

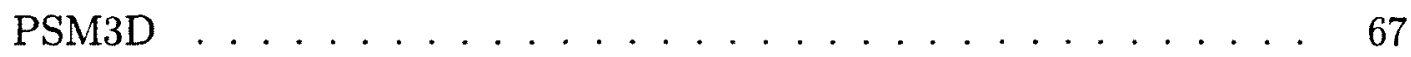

29 Vertical force $\left(F_{z}\right)$ for typical ship motion computed using GRM3D

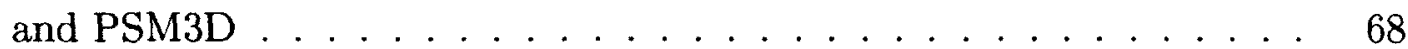

30 Longitudinal moment $\left(M_{x}\right)$ for typical ship motion computed using GRM3D and PSM3D ...................... 68

31 Lateral moment $\left(M_{y}\right)$ for typical ship motion computed using GRM3D and PSM3D ................................ 69 
32 Vertical moment $\left(M_{z}\right)$ for typical ship motion computed using GRM3D

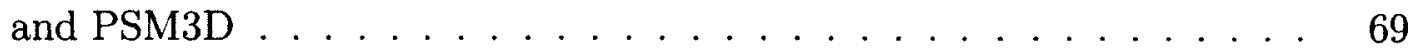

33 Frequency response for the cart model stabilized with a spring and damper in translation for the case where $\zeta=0.17 \ldots . . . .72$

34 Frequency response for the cart model stabilized with a spring and damper in rotation for the case where $\zeta=0.085 \ldots . . . . .72$

35 Spatial inverted pendulum physical model. . . . . . . . . . . . . 79

36 Inverted pendulum custom-designed universal joint. . . . . . . . . . 80

37 Cart load translating base design. . . . . . . . . . . . . . . . 81

38 Solid model of the assembled cart load arrangement. . . . . . . . . . 82

39 Implementation of the tension/compression link with the cart load model. 83

40 Coordinate systems for the experimental apparatus compared to the dynamic model . . . . . . . . . . . . . . . 86

41 Example of breaking moment into force and position vector components 87

42 Longitudinal force $\left(F_{x}\right)$ computed using GRM3D and the load cell results from the experimentation . . . . . . . . . . . . 89

43 Lateral force $\left(F_{y}\right)$ computed using GRM3D and the load cell results from the experimentation . . . . . . . . . . . . . .

44 Vertical force $\left(F_{z}\right)$ computed using GRM3D and the load cell results from the experimentation . . . . . . . . . . . . . . 90

45 Longitudinal moment $\left(M_{x}\right)$ computed using GRM3D and the load cell results from the experimentation ............... 90

46 Lateral moment $\left(M_{y}\right)$ computed using GRM3D and the load cell results from the experimentation . . . . . . . . . . . . . . . . 91

47 Vertical moment $\left(M_{z}\right)$ computed using GRM3D and the load cell results from the experimentation . . . . . . . . . . . . . 
48 Longitudinal force $\left(F_{x}\right)$ computed using GRM3D coupled to pendulum load and the load cell results from the experimentation . . . . . . 93

49 Lateral force $\left(F_{y}\right)$ computed using GRM3D coupled to pendulum load and the load cell results from the experimentation . . . . . . . . 93

50 Vertical force $\left(F_{z}\right)$ computed using GRM3D coupled to pendulum load and the load cell results from the experimentation . . . . . . . . 94

51 Longitudinal moment $\left(M_{x}\right)$ computed using GRM3D coupled to pendulum load and the load cell results from the experimentation . . . .

52 Lateral moment $\left(M_{y}\right)$ computed using GRM3D couple to pendulum load and the load cell results from the experimentation . . . . . .

53 Vertical moment $\left(M_{z}\right)$ computed using GRM3D coupled to pendulum load and the load cell results from the experimentation . . . . . . 95

54 Longitudinal force $\left(F_{x}\right)$ computed using coupled GRM3D coupled to cart load and the load cell results from the experimentation $\ldots . .96$

55 Lateral force $\left(F_{y}\right)$ computed using coupled GRM3D coupled to cart load and the load cell results from the experimentation $\ldots \ldots 96$

56 Vertical force $\left(F_{z}\right)$ computed using coupled GRM3D coupled to cart load and the load cell results from the experimentation . . . . . . . .

57 Longitudinal moment $\left(M_{x}\right)$ computed using coupled GRM3D coupled to cart load and the load cell results from the experimentation . . . .

58 Lateral moment $\left(M_{y}\right)$ computed using coupled GRM3D coupled to cart load and the load cell results from the experimentation . . . . . . 98

59 Vertical moment $\left(M_{z}\right)$ computed using coupled GRM3D coupled to cart load and the load cell results from the experimentation $\ldots . .99$

60 Normal force location to counteract the tipping moments on the block model with a generic footprint . . . . . . . . . . . . . . . . 102 
61 Determining the location of the normal force by the angle between the vertex points on the polygon footprint . . . . . . . . . . 103

62 Stability model presented by Graham for MII detection . . . . . . . . 104

63 Scaled drawing of the MII man assembled apparatus with staggered footprint attached . . . . . . . . . . . . . . 105

64 Scaled drawing of the straight and staggered footprint configurations 112

65 Physical apparatus with staggered footprint attached for the MII stance model validation . . . . . . . . . . . . . . . . . 113

66 Coordinates of the normal force plotted about the straight footprint from simulation ....................... 114

67 Coordinates of the normal force plotted about the staggered footprint from simulation . . . . . . . . . . . . . . . . . . 114

68 Coordinates of the normal force for $25 \mathrm{~cm}$ foot length footprint facing the $x$ direction . . . . . . . . . . . . . . . 116

69 Coordinates of the normal force for $30 \mathrm{~cm}$ foot length footprint facing the $x$ direction $\ldots \ldots \ldots \ldots 117 \ldots \ldots \ldots$

70 Coordinates of the normal force for $25 \mathrm{~cm}$ foot length footprint facing the $y$ direction . . . . . . . . . . . . . . . . . . . 118

71 Coordinates of the normal force for $30 \mathrm{~cm}$ foot length footprint facing the $y$ direction . . . . . . . . . . . . . . . . . . 119

72 Coordinates of the normal force with a $75 \mathrm{~cm}$ wide straight stance . 120

73 Coordinates of the normal force with a $100 \mathrm{~cm}$ wide straight stance . 120

74 Diagram of angle conventions for the stance angle and ship heading angle121

75 Optimum stance with a ship heading of 30 degrees . . . . . . . . . 122

76 Trend of the optimum stance angle against ship heading relative to the wave direction. . . . . . . . . . . . . . . . . . . . . . . . . . . 122

77 Coordinates of the normal force with no load attached . . . . . . 126 
78 Coordinates of the normal force with the pendulum load attached . 127

79 Coordinates of the normal force with the cart load attached $\ldots \ldots 128$ 


\section{List of Symbols}

\begin{tabular}{ll}
\hline Symbol & Definition \\
\hline Vectors & translational position \\
$v$ & translational velocity \\
$a$ & translational acceleration \\
$\theta$ & rotational displacement \\
$\omega$ & rotational velocity \\
$\alpha$ & rotational acceleration \\
$F$ & force vector \\
\hline Matrices & rotation matrix from coodinate system A to coordinate system \\
&
\end{tabular}




\section{Superscripts}

$\begin{array}{ll}\text { IN } & \text { described in the inertial coodinate frame } \\ \text { described in the ship coordinate frame } & \text { described in the model coordinate frame } \\ \text { TO } & \begin{array}{l}\text { described in the coordinate system at the attachment point } \\ \text { of the second mass }\end{array}\end{array}$

\section{Subscripts}

A External applied force (with F vector); Attachement point on ship deck (All other vectors)

$A x \quad$ External applied force in $x$ direction (with $\mathrm{F}$ vector); attachment point on ship deck in $x$ direction (All other vectors)

Ay External applied force in $y$ direction (with $\mathrm{F}$ vector); attachment point on ship deck in $y$ direction (All other vectors)

$A z \quad$ External applied force in $z$ direction (with $\mathrm{F}$ vector); attachment point on ship deck in $z$ direction (All other vectors)

$B \quad$ Centre of gravity of mass 1

$B x \quad$ Centre of gravity of mass 1 in $x$ direction

By Centre of gravity of mass 1 in $y$ direction

$B z \quad$ Centre of gravity of mass 1 in $z$ direction 


\begin{tabular}{|c|c|}
\hline$C G$ & Centre of gravity of entire mass \\
\hline$C G / A$ & Centre of gravity with respect to point $\mathrm{A}$ \\
\hline$x$ & $x$ component of reaction force or moment at point $\mathrm{A}$ \\
\hline$y$ & $y$ component of reaction force or moment at point $\mathrm{A}$ \\
\hline$z$ & $z$ component of reaction force or moment at point $\mathrm{A}$ \\
\hline$R$ & Point of attachment of mass 2 \\
\hline$R / A$ & $\begin{array}{l}\text { Position of point } R \text { with respect to the attachment point to } \\
\text { the deck }\end{array}$ \\
\hline$R / B$ & $\begin{array}{l}\text { Position of point } R \text { with respect to the centre of gravity of } \\
\text { mass } 1\end{array}$ \\
\hline$T / R$ & $\begin{array}{l}\text { Position of centre of gravity of mass } 2 \text { with respect to point } \\
\mathrm{R}\end{array}$ \\
\hline$T R$ & Reaction force from mass 2 onto mass 1 \\
\hline$T R x$ & Reaction force in the $x$ direction from mass 2 onto mass 1 \\
\hline$T R y$ & Reaction force in the $y$ direction from mass 2 onto mass 1 \\
\hline$T R z$ & Reaction force in the $z$ direction from mass 2 onto mass 1 \\
\hline$B R$ & Reaction for from mass 1 at the attachment point \\
\hline$B R x$ & $\begin{array}{l}\text { Reaction force in the } x \text { direction from mass } 1 \text { at the attach- } \\
\text { ment point }\end{array}$ \\
\hline
\end{tabular}


Reaction force in the $y$ direction from mass 1 at the attachment point

$B R z \quad$ Reaction force in the $z$ direction from mass 1 at the attachment point

1

Translating mass

2

Rotation mass 


\section{Chapter 1}

\section{Introduction}

\subsection{Motivation}

Shipboard postural stability affects crew safety, the time required to complete specific tasks, overall ship effectiveness, and ultimately ship design. Postural stability has been quantified using the rate of motion-induced interruptions (MIIs) where MIIs are defined as events where a person must focus attention on maintaining balance as opposed to the task at hand either my adjusting stance or mechanically bracing against deck motion. Existing models used for predicting MIIs are relatively simple. In particular, they assume unrealistically simple stance geometry and ignore the disturbing influences of unsteady external loads with which the simulated crew member may be interacting. Such loads could include penduluating loads such as a small boat suspended from a crane or davit, an example of which can be seen in Figure 1; also loads constrained to the plane of the deck such as carts, pallets, and trolleys.

Recognizing the importance of better understanding of postural stability while interacting with unsteady shipboard loads, a collaborative project was undertaken between Defence Research and Development Canada and Carleton University to in-

vestigate this problem. In the overall project, two conventional postural stability 


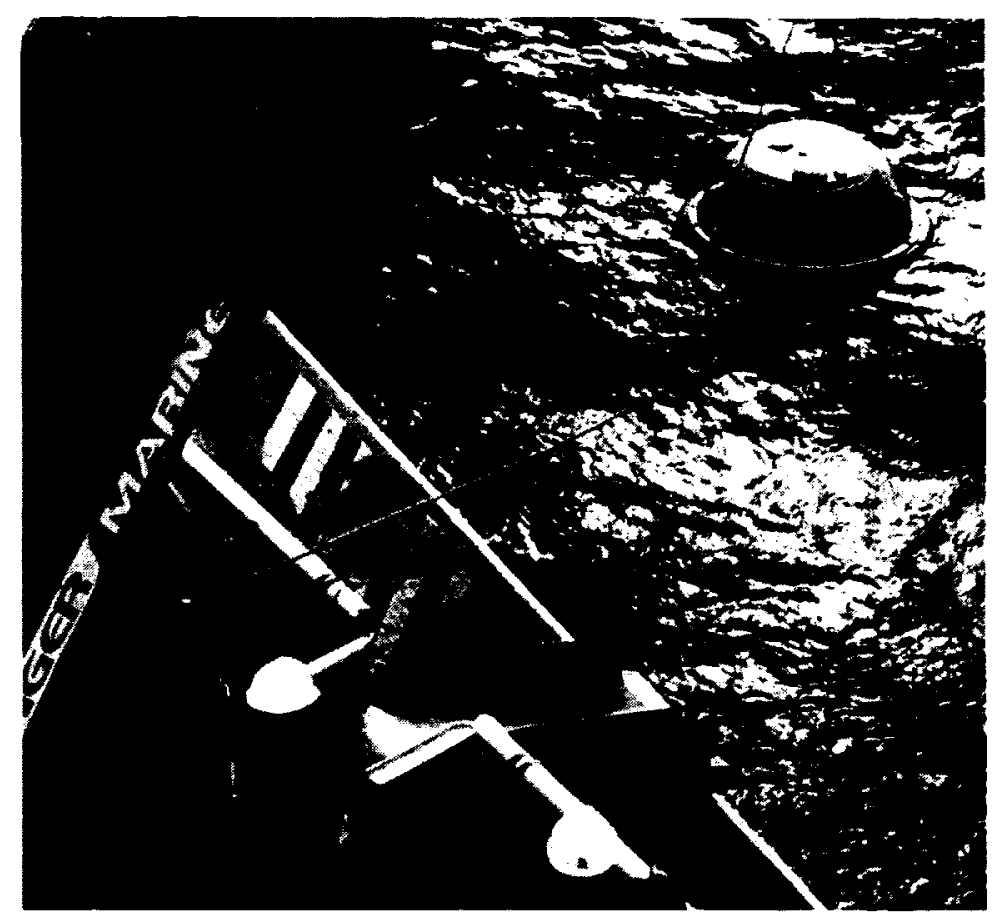

Figure 1: Crew member handling a wave buoy suspended by a crane.

models were generalized: a Graham rigid body model and a spatial inverted pendulum model. Two unsteady load models were also investigated: an in-plane cart load and a penduluating load; as well as development of a coupling model used to connect the postural model to the load model. A monolithic and a distributed simulation environments was created that implemented these models. A series of physical experiments was also designed, built, and conducted to validate the developed models.

This thesis focusses on those aspects of the overall project that were completed by the author. These comprise of the generalization of the rigid body Graham model, development of a two-degree of freedom unsteady in-plane cart load model, implementation of all models in the distributed Virtual Flight Deck-Real Time (VFD-RT) simulation environment [1], and validation of the developed models through the physical experimentation. The research presented in this thesis expands the existing body of knowledge by generalizing a classical model into three dimensions, incorporating 
the effects of an external load, and generalizing model stance geometry.

\subsection{Literature Review}

Humans are required to cope with a wide range of postural stability conditions ranging from deceptively simple tasks such as quiet standing to more complex dynamic actions such as walking and running. Over the years many models to describe human postural stability have been developed. These models vary widely in complexity, ranging from single segment inverted pendulums up to fully articulated multi-link skeletons [2]. Initially these models were developed with the goal of understanding the human sense of balance; however, more recently they have been identified as a potential tool in quantifying the effects of motion environments on human performance. This section presents a review of current and past research on human postural stability in a motion environment. It will begin by briefly explaining the biological systems which make up the human postural control system. Having explored human postural stability research from the theoretical perspective, the applications of this knowledge will then be investigated. This includes quantifications of the risks related to working in a motion environment and the application of biomechanical principles to assessing the postural stability challenges related to the shipboard motion environment.

\subsubsection{Physiology of Human Postural Stability}

The human body is inherently unstable due to the height of its centre of mass [3]. Consequently, constant minor postural adjustments and muscular torques are required to simply maintain an upright posture. Basic tasks such as quiet standing require an elaborate scheme of biological systems and algorithms for proper execution [3]. The human body contains three primary sensory mechanisms that are used during postural control: vision, proprioception, and the vestibular system. Each system provides a 
unique set of information related to the surrounding environment and the body's motion. This information is processed by the central nervous systems in order to form an overall postural state which is then used to determine an appropriate mechanical response [3]. Each of these systems is most sensitive to a different set of postural disturbance types $[3,4]$. As such, the relative importance of any individual system in the overall maintenance of postural stability varies relative to motion characteristics such as amplitude, frequency, and the available sensory information [4].

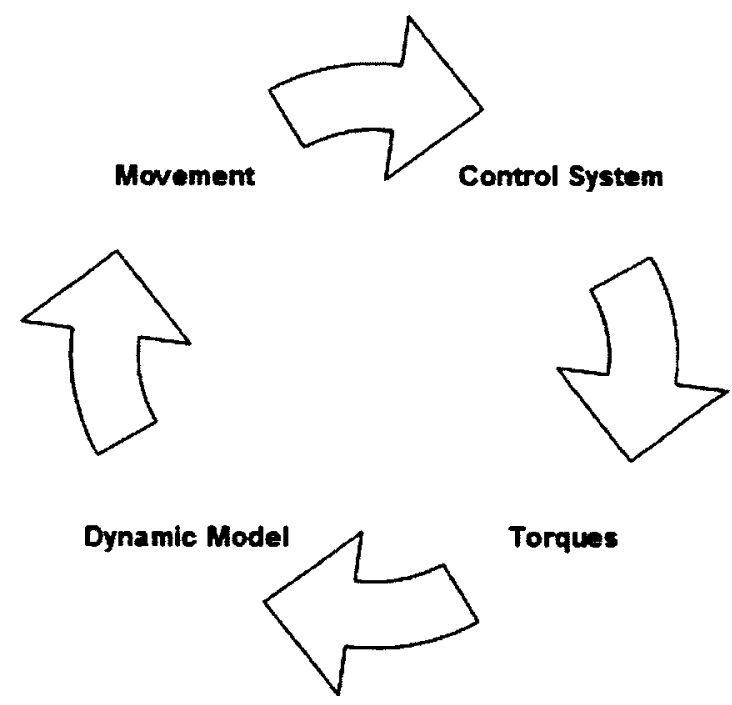

Figure 2: Feedback postural stability model.

The typical approach to studying the biological control system which interprets these sensory inputs is to represent it as a feedback model. Kinematic variables of the body, as would be sensed by the three previously mentioned systems, are input to a control block. This block then calculates the necessary torques and forces to be applied by the body's muscles to its various segments. The corresponding movements of the body can then be generated through the implementation of a dynamic model which then completes the control loop by passing these calculated motions back to the control block as shown in Figure 2 [2].

More recent investigations have demonstrated that the postural control system is 
likely composed of both feedback and feed-forward elements. It has been proposed by some researchers that the body's postural system incorporates a finely tuned set of predetermined reflexive postural responses. And although the central nervous system continuously receives postural state information, there is evidence that supports the hypothesis that it does not actually engage in postural control unless disturbances exceed a specific threshold $[5,6]$. This serves to simplify the overall postural maintenance task in relatively stable environments and to compensate for time delays related to data transmission and feedback control decision processes.

\subsubsection{Postural Stability Modelling}

Postural stability models are models that define the dynamics of the human body [7]. Several of these models have been used to define the stability of humans during a walking gait and quiet standing. Though these models are used for different applications, dynamic postural models can be used for either application.

In 1972, Chow et al. at Harvard University developed a stability model for a torso [8]. The model was to be used as the torso section of a more complex model for biped locomotion. This model consisted of a three-rotational-degrees-of-freedom single inverted pendulum. The equations of motion were derived using Lagrange's equation. The model allowed for the position of the base to follow a perscribed trajectory over time.

In relation to locomotion, Gubina et al. in 1974 developed a single link inverted pendulum model with two moving massless legs modelled with force generators [7]. This model was constrained to the sagittal ${ }^{1}$ plane and modelled the torso dynamics supported by the two massless legs. The knee was modelled through the changing

\footnotetext{
${ }^{1}$ Sagittal plane is a vertical plane which passes from ventral (front) to dorsal (rear) dividing the body into right and left halves.
} 
length of the legs and force generators to supply the torque. This model was also derived using Lagrange's equation. The equations were solved for the two translational degrees of freedom and one rotational degree of freedom of the torso.

In 1975, Murray et al. introduced a different modelling method for postural stability [9]. Unlike the pendulum models that were based more on physical geometry of a human, Murray et al. suggested that a model could use the location of the centre of pressure on the surface and the distance it is within the stance. The fluctuations of the location of the centre of pressure were plotted and the variations in the position determined a relative steadiness. The validity of this type of modelling was checked by performing experiments with subjects standing on a force plate and asking them to perform various standing tasks. It was found that these fluctuations of the centre of pressure location depend on the age of the individual.

Golliday et al. in 1976 developed a two-link inverted pendulum model designed for human locomotion [10]. Unlike the model derived by Gubina et al., the legs in this model included mass and inertia. The equations of motion were again derived using Lagrange's equation. Their model is illustrated schematically in Figure 3.

Postural stability models are dynamically unstable due to the fact that humans are dynamically unstable. This requires that there be a control system in order to maintain stability of the system. As a result, control system analysis is one of the applications for which postural models have been developed. For these applications the models do not need to be complex. In 1976 Hemami used a single-link inverted pendulum model to determine applicable control algorithms [11].

In 1978, Hemami et al. developed a three-link inverted pendulum postural model [12]. This model was constrained to the saggital plane and had three links attached with revolute joints, as shown in Figure 4. This model was introduced to determine motions for several different tasks including sitting down, bending, and standing up. The model was derived using Newton-Euler dynamics and linearized 


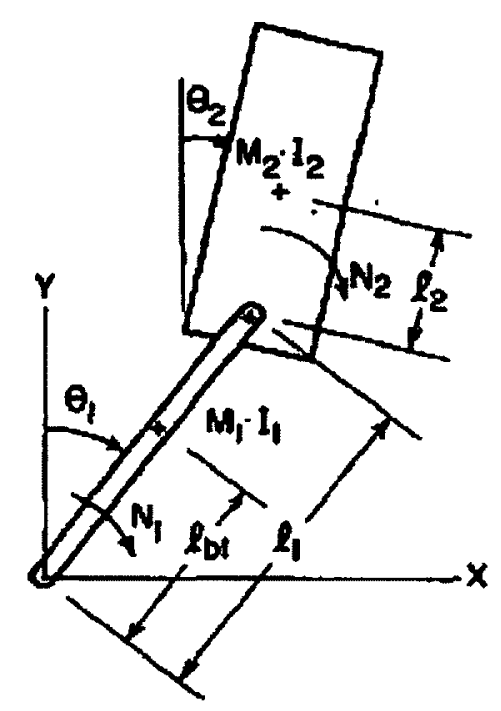

Figure 3: Golliday et al. two-link inverted pendulum model [10].

about the applicable operating point. This model could be used to recreate typical human motions by applying an iterative solution method.

Hemami et al. also conducted research with another team in 1978 to investigate a vestibular model. Humans control their posture using the vestibular system, which relies heavily on otoliths and the semicircular canal within the ear [13]. The vestibular model that was used in 1978 was derived by Nashner [14]. This model of the vestibular system is defined in the frequency domain as a set of transfer functions modelling the effects from the two different parts of the vestibular system: the otoliths and semicircular canals. The vestibular model was initially applied to a single link and then a double link inverted pendulum postural model for control. It was found that the vestibular model could be used to successfully control the pendulum models.

Experimentation can be quite costly and time consuming. Mathematical models have been created to minimize the extent of required experimentation. In 1980 , Koozekanani et al. developed a physical model in order to determine the centre of pressure of a four-link inverted pendulum with a foot link [15]. This model was not 


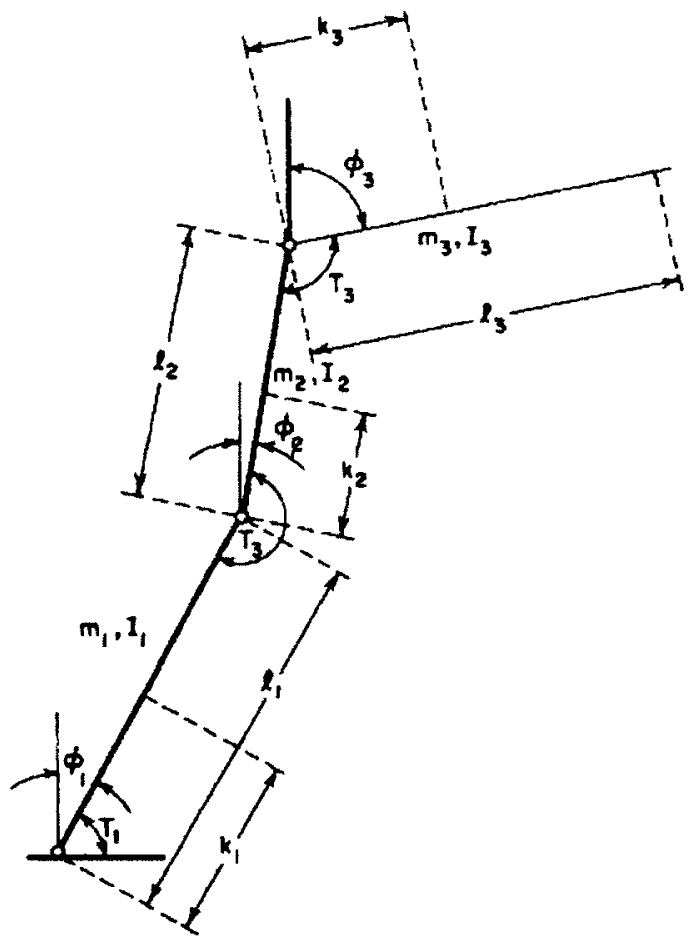

Figure 4: Hemami et al. three-link inverted pendulum model [12]. 
used for postural stability purposes on its own, but could be used with centre of pressure models for stability. This model did not have a control system; therefore, in order to calculate the centre of pressure all the link properties, including applied forces and torques, had to be known. Also, this model was limited to the sagittal plane. The model can be seen in Figure 5 .

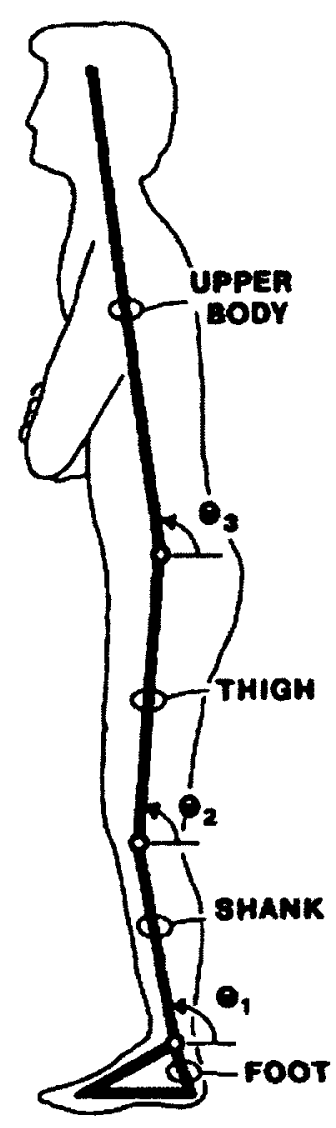

Figure 5: Koozekanani et al. four-bar linkage model [15].

In order to extend the Koozekanani et al. model to more of a postural stability model, in 1981 Stockwell et al. provided extensions to the model [16]. It was extended with another linkage to represent the head motions and allow for more control based on the location of the centre of pressure of the linkages. Experimentation was 
conducted with human subjects in order to observe the postural sway effect. Stockwell suggested that a four-link model should be sufficient to model the postural sway motions.

In 1982, Hemami et al. developed a spatial inverted pendulum model for use in locomotion studies [17]. The pendulum model was used to represent the torso motion for gait analysis. The model was derived using the Newton-Euler method. There are three constraining forces that act at the base attachment point of the linkage. An interesting by-product of the model is that this model not only describes the behaviour while attached at the base, but also indicates when the link may slide or leave the ground. One of the assumptions used for this analysis was that the rotation about the linkage axis is constrained by two possible types of constraints. There is either a hard constraint which the body cannot pass and a soft constraint which can be passed slightly. These constraints are modelled as a hard stop and as a spring/damper which restrict arm motion.

By 1984, Hemami et al. developed a model with a more biological basis. The models discussed thus far have all had rigid connections between the links in the models in order to lower the dimensionality of the system [10]. However, human linkages, for example the knee, are not rigidly attached [18]. For this reason Hemami et al. extended a two link model with an interface at each joint that could be used to represent tissue effects. The model can be seen in Figure 6. Model stability was obtained using Lyapunov stability theory but certain constraints would not provide valid answers for the interface forces [18].

Peeters et al. ,in 1985, solved the single-link inverted pendulum model in the frequency domain and observed the spectral response of the system [2]. This analysis was performed to find the relationship between the motion of the body and the torque at the ankle joint. The results presented spectra in the frequency domain based on the torque. 


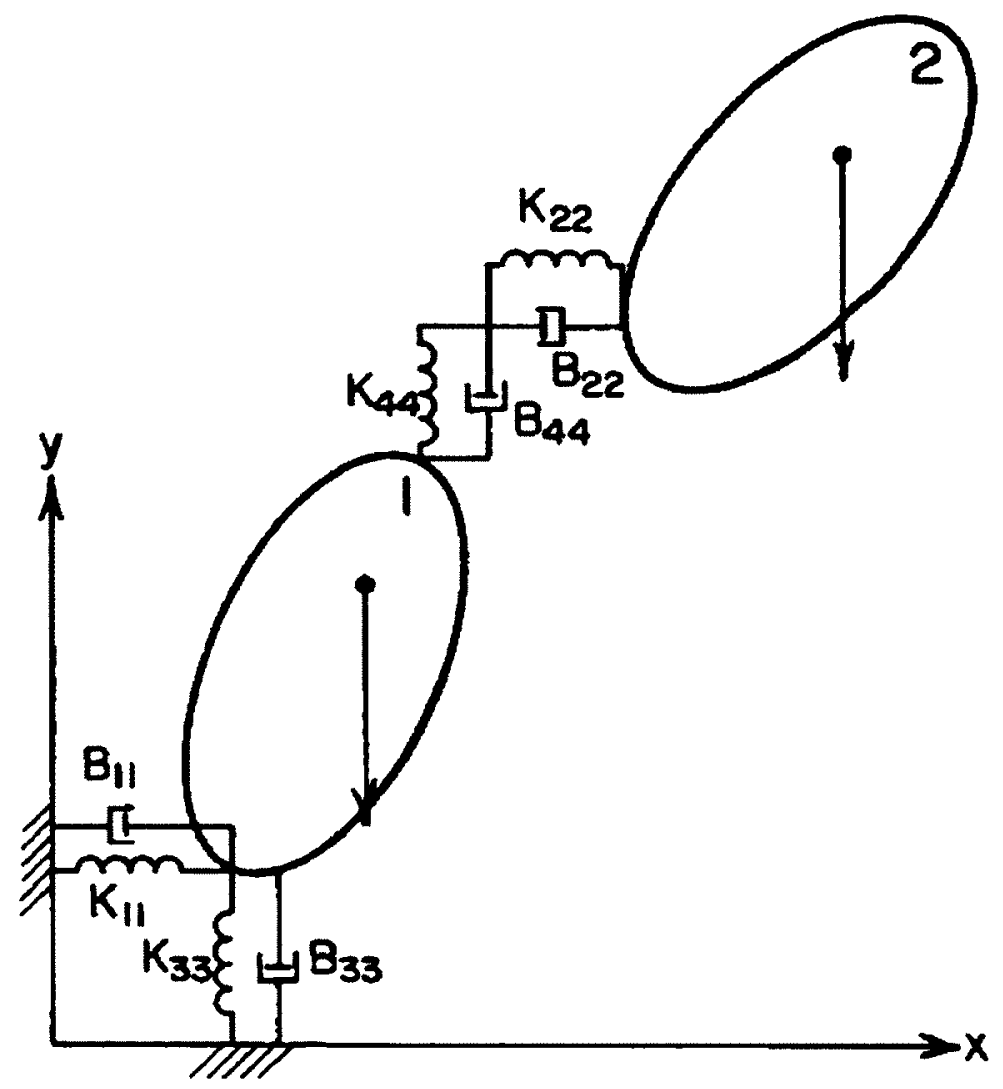

Figure 6: Hemami et al. two-link inverted pendulum model with interface forces [18]. 
A further development of the inverted pendulum model was achieved in 1989 by Barin. Barin created a linkage model that would allow for an arbitrary number of links in the system [19]. This model was also constrained to the sagittal plane. The model can be seen in Figure 7. This model was developed in order to have a multiuse model as long as all the link and joint properties are known for a particular application.

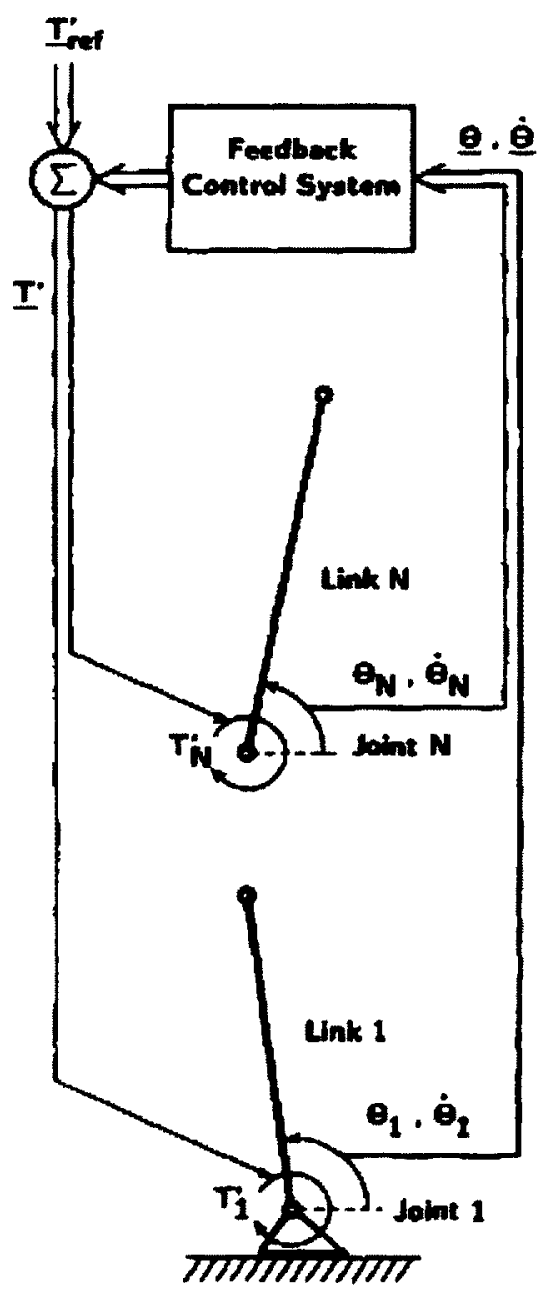

Figure 7: Barin multilink inverted pendulum model [19].

Riccio et al. researched the effects of dynamic motion and the stability of humans. This research was mostly qualitative and was observed through experimentation [20]. 
In previous pendulum models the orientation was set by a kinetic variable, the direction of the gravitational force [21]. It is stated that the definition of balance of a human could be more dynamically defined given that the vestibular system is affected by inertial effects. This would provide insight into postural stability models with control systems. In 1993, Riccio performed a series of experiments that submerged subjects in water in order to quantify the effect of these dynamic effects on human balance [22]. The dynamic effects were quantified as a relative tilt to the gravity vector after having undergone dynamic motions. It was found that the dominance of balance over gravity was related to the tilt angle.

The postural stability models presented thus far have all been applied in the sagittal plane. In 1993, Iqbal et al. presented a model in the frontal plane, as seen in Figure 8. This model is a four-link model having one link for each leg, one for the pelvis, and a fourth for the torso [23]. From the figure it can be seen that the right leg is not constrained to the ground. Each of the joints is a revolute joint such that this model can investigate the rotation at the hips as well. The model was solved using perturbation methods and Taylor series expansions. This model was used to investigate control strategies of voluntary tasks as well as the sway motion effect.

Patton et al. , during the period from 1997 through 1999, developed a series of experiments in order to determine the effects of the coordinates of the centre of mass within the base support geometry [24]. His experiments also investigated the effects of the velocity of the centre of mass and centre of pressure. The desired result from these experiments was a threshold value which would determine whether a person would fall or remain standing after a perturbation. The model used to investigate this was the single-link inverted pendulum model. The centre of mass and centre of pressure are known and using the values obtained from the experiments, thresholds for stability were found [25]. Applying these to the pendulum model determined if a person would remain standing after the perturbation. 


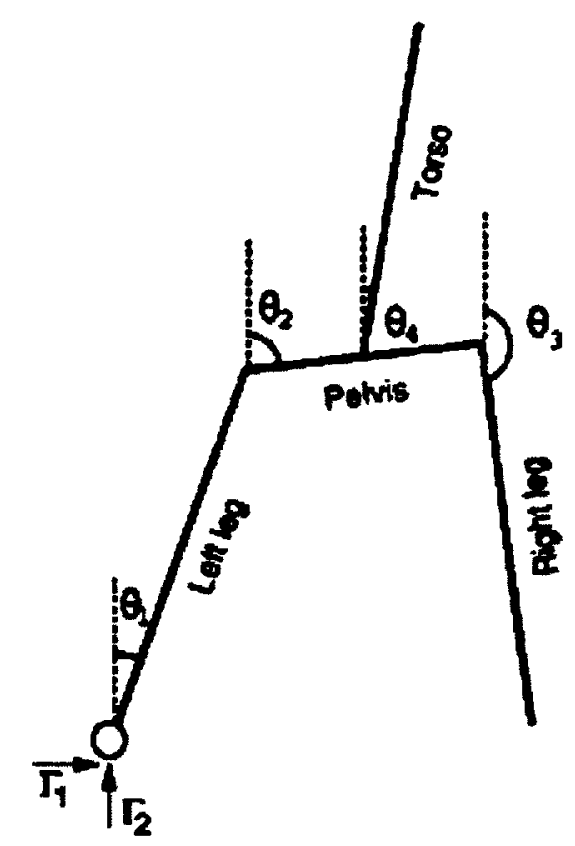

Figure 8: Iqbal et al. frontal postural stability model [23].

In 1998, Slobounov et al. performed similar experiments to Patton but took into account the effect of age on the stability thresholds. This study did not compare the results to a postural stability model and were only found to be threshold values for other models [26]. Subjects varying in age from 60 through 92 were tested in order to determine how age affected the threshold of centre of pressure values within the stance width. It was found that the higher the age, the lower the threshold is, which decreases the motion severity that will cause instability.

Wu et al. developed a single link inverted pendulum model with two rotational degrees of freedom in 1998 which allowed for a free moving base [27]. The base attachment point was modelled to be able to move in any direction. The only limitation was that if there was acceleration it must remain constant. This model was designed solely as a mathematical model that could be used for other postural stability applications, such as trunk stability for locomotion [27]. The model was derived using 
Lagrangian dynamics. In their paper the model was used to test control methods for the inverted pendulum. By $2000 \mathrm{Wu}$ et al. had introduced a new postural stability model. The model was a planar two-link inverted pendulum model [28]. The base of the pendulum was able to move freely. The same limitation on the base motion applies, where the acceleration must be constant.

Hemami et al. , in 2006, modelled a three-link inverted pendulum in the sagittal plane that was attached to a motion platform. The model also included muscle models for the torques applied at the ankle, knees, and hips as can be seen in Figure 9. Several experiments were conducted with subjects in order to validate the model. The results from the pendulum experiments were found to be similar to the calculated model results. The calculated forces and moments from the model had smaller magnitudes than those from the corresponding human experiments.

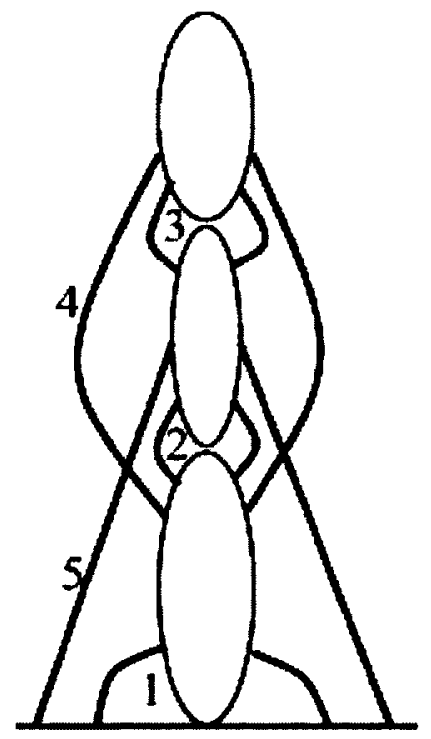

Figure 9: Hemami et al. muscle model for three-link pendulum [29].

In 2006 Nawayseh et al. performed experiments on the effect of oscillations on the postural stability of standing persons. A centre of pressure location model was used 
to determine the stability of a person. The experiments indicated that the higher the frequency of the oscillations the more likely the person was to fall.

Another method for determining the effect of the centre of pressure on stability was introduced by Schmid et al. in 2007. This method used a time to boundary method on four different parameters to analyze the effect on stability [30]. The time to boundary function estimated the predicted time when the centre of pressure trajectory would cross the boundary thresholds, using a parabolic function containing position, velocity, and acceleration of the centre of pressure data. It was found that this method allows for the postural stability to be maintained.

A recent study performed in 2010 by Humphrey et al. used a three-link planar inverted pendulum model [31]. The model used a muscle model for joint actuation as well as a vestibular model for feedback control. This model was used in the study of centre of pressure and centre of mass movements.

\subsubsection{Motion Induced Interruptions}

Biomechanical postural stability models were initially developed with the goal of understanding the human sense of balance; however, more recently they have been identified as a potential tool in quantifying the effects of motion environments on human performance. For example, crew members working in a shipboard motion environment are required to perform a variety of physically and mentally demanding tasks such as walking, weapons loading, and lifting [32]. If the ability of the crew to complete these tasks is in any way impaired, the overall efficiency of the crew member decreases resulting in potential increased costs and decreased effectiveness. Also, this may cause the crew member to become so impaired that their personal safety may be at risk. A concept to quantify the performance degradation caused by the crew members adjusting balance was introduced by Baitis and Applebee in 1984 [33]. The performance of a crew member is said to be reduced if the person has 
a motion induced interruption (MII) which is defined as an incident when they must take a step, grab a hold, or stop what they are doing in order to maintain balance [34]. The MII concept not only considers the motion of the environment but the effects of this motion on humans who are being analyzed. Thus biomechanical models supply a means by which the human response can be predicted for a given set of motions in order to provide operational and design information.

Biomechanical models are used in order to observe the effects of the motion environment on the postural response. These models however were not designed in such a way to observe the possibilities of MIIs. Several different postural models have been developed for the purpose of determining the MII events specifically.

The Graham rigid body model was first introduced in the early 1990's [35]. The model provides a mathematical approximation of the possible inertial causes of an MII. The model is based on a block having humanoid mass, inertia, and support base properties. An MII is said to occur if the block either has a sliding event or a tipping event. The occurrence of these events is identified by exceeding thresholds based on the properties of the block and gravity. A sliding event is said to happen if the lateral inertial forces and gravity acting on the block exceed the opposing frictional capacity. The frictional capacity is calculated as the force normal to the flat surface multiplied by the frictional coefficient applicable between the surface and the block. The lateral forces are found by a summation of the forces parallel to the flat surface. Initial tests conducted through observation of an unoccupied chair subject to ship motion indicated relatively good agreement between predicted slides for the chair and the actual slides observed. This is to be expected since the chair is simply a rigid body which has no dynamic characteristics to be accounted for [35]. A tipping event is said to occur if the moment about one of the model's feet falls to zero, and can be seen in Figure 10. This tipping model is used to predict when a person will require to take a step or grab a support to retain balance. These two thresholds are 
used to model when a person is most likely to take action in order to retain balance. These thresholds can be used in simple models such as the block on a deck, or include unsteady wind loading, or even be used in articulated models which define the person not as a simple block but an inverted pendulum or other more complex models.

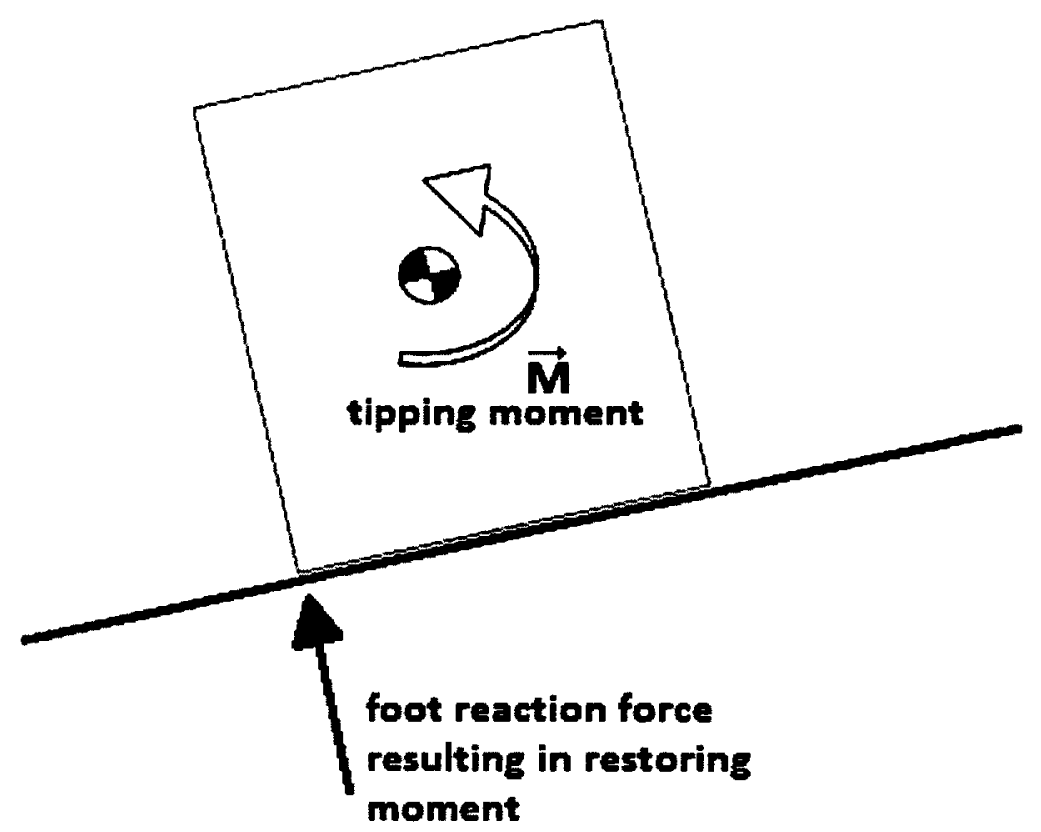

Figure 10: Moments on the Graham MII Model.

A research initiative established by the American, British, Canadian, and Dutch (ABCD) Working Group on Human Performance at Sea to explore human factors within the shipboard environment resulted in an extensive set of experiments to investigate the performance characteristics of Graham's model. During the early 1990's a large data set on human performance in the shipboard environment was produced at the Naval BioDynamics Laboratory (NBDL) in New Orleans. The experiments subjected 15 participants to two levels of ship motion severity using a large ship motion simulator platform. The subjects were required to complete a number of tasks during the motion profiles. Human postural response data from the tasks consisting 
of standing facing port and standing facing aft were used by Lewis and Griffin to check the validity of the rigid body model and to investigate the potential application of more complex models to MII prediction such as parametric methods [36]. From these experiments it was proposed that Graham's model could be tuned to more accurately predict MIIs by empirically choosing tipping and sliding thresholds to match experimental MII occurrences. A similar adjustment process was recommended for parametric stability models. Initially, for a parametric model, MIIs were defined as points at which the centre of pressure exceeded base of support limits. In practice the usable base of support region was found to be smaller than the theoretical maximum value. Based on this, it makes sense to adjust the parametric model's MII threshold accordingly.

A second series of experiments based on the initial NBDL investigations was conducted by the United Kingdom's Defence Research Agency using a large motion simulator [37-39]. The ability of the simulator used in this case to provide motion cues in five degrees of freedom provided the opportunity to generate a set of postural response data relating to frontal plane MIIs. During the experiment subjects were required to complete several different tasks such as walking, weapon loading, and standing while being subjected to the NBDL motion profiles. As suggested by the NBDL experiments, empirical MII thresholds were determined for the rigid body MII model. In addition to the standing tasks, empirical model thresholds were determined for all of the experiment tasks despite the fact the model does not physically represent them. The tuned model was found to provide good predictions of MII occurrences in all cases although it underpredicted at high MII rates. A statistical model of MII occurrences was also investigated using the experiment data.

Experiments performed in 1993 by McIlroy and Maki investigated the psychological effect of either allowing someone to step to maintain balance or not allowing them to step [40]. The experiments conducted applied a perturbation in a single direction 
and the subject was either told they could or could not step. The results of the experiments determined that if a subject was allowed to step, the occurrences of stepping incidences increase as compared to not being allowed to step. This shows that there is a psychological effect when considering MII occurrences. In 1997 Maki and McIlroy investigated the likelihood of a person maintaining postural stability by using fixed stance methods versus non-fixed stance methods [41]. The fixed stance methods included bending the knees, rotating the hips, applying a moment at the ankles, and similar methods. Non-fixed stance methods included grasping an external support or taking a step. The results of the experiment showed that the onset of non-fixed stance recovery methods occur well before the centre of mass has reached the stability limits. This implies that a person is likely to step well before the stability limits are reached.

A method of articulated postural modelling was investigated by McKee using a two-plane articulated model [42]. The model is defined by using a single degree of freedom inverted pendulum in the sagittal plane and a four-bar linkage in the frontal plane, as shown in Figure 11. This model also used controllers for the ankle joint moments which were tuned based on experimental data recorded at the Naval Biodynamics Laboratory [43]. It was observed that the biodynamic model was predicting a slightly lower number of MII events than observed where as the untuned Graham model overpredicted the number of MII events. This introduced the possibility of articulated models being a more accurate approach for determining MII events [42].

An expanded inverted pendulum model for modelling postural stability in a shipboard application was introduced by Langlois in 2010 [44]. The pendulum model was a three-dimensional spatial model as opposed to some classical models that were limited to two dimensions. This model was also designed with the intention of predicting MII events. The model was derived by separating the translational and rotational 

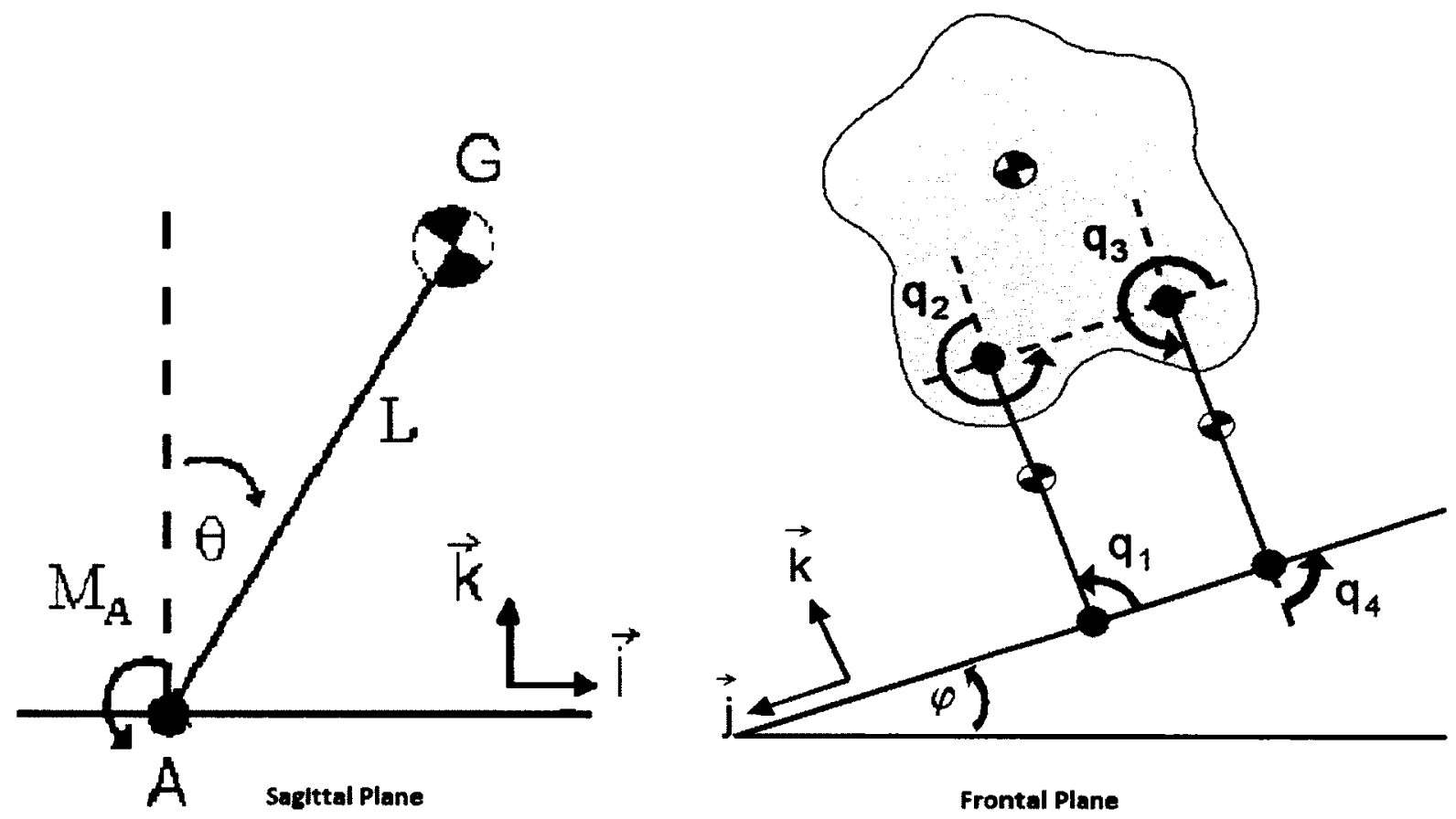

Figure 11: Frontal and sagittal plane models for McKee and Langlois's articulated postural model [42]. 
components. The translational components are solved in order to find the forces acting at the base of the pendulum. The rotational components are solved using Euler's equation to determine one unknown ankle reaction moment and the angular acceleration of the pendulum. A controller is necessary to command two ankle torques and stabilize the system. The controller is set with applicable damping in order to maintain a vertical stance with respect to the gravity vector while predicting MII rate and times of occurrence based on available experimental data. MII detection in this model is based on the two criteria defined in Graham's model. The sliding case is defined by a sliding threshold which is a function of the friction coefficient and the vertical and lateral forces. A tipping case is defined by a tipping threshold which is a function of the moment and the forces acting on the body. The model was verified using a controller tuned to the MII data from experience with motion platform and available sea trial MII data. The model was verified for a single articulated segment and two revolute degrees of freedom.

Duncan et al. at Memorial University of Newfoundland ran a series of experiments on MII occurrences at sea $[45,46]$. The experiments performed in 2009 used 11 participants from naval personnel in order to investigate the effect of thoracolumbar kinematics and foot centre of pressure on postural stability. The experiments required the subjects to perform two different tasks in different sea conditions. The tasks were a neutral standing task and a standing task with their feet shoulder width apart holding a stationary $10 \mathrm{~kg}$ load. The data collected for this experiment were the thoracolumbar kinematics, the velocity of the centre of pressure of the individual foot, and video subsequently used to identify MII occurrences. It was found from these experiments that during MIIs, the sudden postural adaptations resulted in significant increases in mean and peak thoracolumbar velocities. This showed that in order to maintain balance a combination of body and foot movements are needed. It also showed that the direction that the subject was standing also affected the number 
of MII occurrences.

Experiments performed by Hasoon et al. at the University of Massachusetts were designed to investigate the response of the subject as if they were an inverted pendulum [47]. The experiment was designed such that the subject was attached to a flat board in order to limit the motions in the sagittal plane. The only control of balance strategy that the subjects could use, based on the apparatus, was the ankle strategy. The subject was then perturbed from the aft by varying degrees and it was observed whether the subject had to step to maintain stability. The results from the experiment were compared to pendulum model developed by Hof et al. [48]. The analysis of the data provided approximation curves of the centre of mass acceleration versus the severity of the perturbation.

Bourgois et al. from Carleton University Applied Dynamics Lab performed postural stability experiments aboard Canadian Forces Auxiliary Vessel (CFAV) Quest. Quest is operated by DRDC Atlantic for experimentation at sea. The 76 metre long vessel was designed to be very quiet for acoustic testing as well very stable for heavy weather trials. The sea trial was from November 20th 2012 to November 28th 2012 and had seas with waves upwards of 5 metres. For the experimentation the human subjects were asked to stand at an angle to the bow and perform a simple logging task using either a clipboard or a tablet. The results from the study will be used to develop control schemes to model human posture in shipboard environments. The task research will be used to determine the effectiveness of hard copy logging versus electronic logging in shipboard environments.

In summary, considerable research has been conducted aimed at understanding and modelling human balance. This work formed the basis for subsequent attempts to model the rate of motion-induced interruptions in shipboard environments. While current MII modelling approaches continue to be refined, simple models are available and are used for operational planning and ship design. In all cases these models 
consider an individual that is only perturbed by ship motion. In practice, shipboard personnel are interacting with external unsteady loads that could potentially adversely affect their postural stability.

\subsection{Overview}

This thesis presents the contributions made to a postural stability modelling (PSM) project in order to determine the effects of external loads on human postural stability in shipboard environments. In the following chapters a postural stability model based on the Graham stability model which additionally allows for a timevarying external load is developed, as well as a two-body cart load model. These two models can be run separately or coupled using an axial spring/damper interface force model. The models are implemented in a Fortran simulation environment in order to computationally solve the models. The Fortran simulation is based on a simulation created by Langlois for the inverted pendulum model (PSM3D) which can also be run with the models developed in this report in addition to an unsteady pendulating load model developed by Langlois (PND3D). In order to create a more robust simulation that can be easily interfaced with many other existing shipboard simulation applications, the models were implemented in a distributed simulation framework named the VFD-RT. The monolithic Fortran simulation was compared with the VFD-RT simulation to verify the simulations. Monolithic in this case means that all models are contained in a single executable. The following chapter presents the results from a series of physical validation experiments. For the experimentation, physical models were fabricated in order to represent the conceptual models. The apparatus was developed as part of the broader PSM project and a brief explanation on their construction is provided. The experimentation included placing the experimental apparatus on a six-degree-of-freedom motion platform and exciting the physical models 
with the motion base. The parameters used in the physical experiments were then used to run matching simulation cases. The results from the physical experiments were then compared to the results from the simulation. The models were validated separately and coupled with the various load models using five motion profiles. The models were thus validated from the physical experimentation. The next chapter defines the concept of an MII as presented by Graham, and expands the definition similar to Langlois [44]. The definition of an MII is then further refined to incorporate an arbitrarily-shaped footprint. Further physical experimentation was conducted in order to validate the MII models presented. The physical experiments included the construction of a three-dimensional Graham model body with contact switches positioned around the base. The switches undepress when tipping MIIs occur such that the direction of tipping and the number of tips can be counted. Simulations were run using the same motion files as the physical experiments for validation purposes of the MII model. The MII model was also validated from the data collected on the Quest sea trial. The effects of stance geometry and unsteady loads on shipboard postural stability were then investigated by running simulations with the two load models for different headings and sea states. Concluding remarks are made in the final chapter. 


\section{Chapter 2}

\section{Model Development}

\subsection{Three-dimensional Graham Model}

The original planar Graham formulation used the conceptual model of a block positioned on the deck of a ship. The corresponding three-dimensional model developed here uses the same basic conceptual design. A schematic representation of the model is shown in Figure 12. The three-dimensional block, with height $h$, length $l$, and width $w$, is placed in a virtual motion environment. The equations governing motion of the model are derived and solved for the interface forces and moments at the attachment point with the deck.

Figure 12 also shows the coordinate systems used in its derivation. These coordinate systems are similar to those found in the Langlois inverted pendulum derivation [44]. The inertial frame (designated $I N$ ) is defined outside of the motion environment. The ship coordinate system (designated $S H$ ) is attached to the ship at the point of attachment of the Graham model and is aligned with the inertial frame in the absence of ship motion. In the ship frame the $x$ direction points to the bow, the $y$ direction points to port, and the $z$ direction points upwards normal to the deck. Finally, a model coordinate system (designated $M O$ ) is attached to the block and located at the interface between the block and the ship deck. 


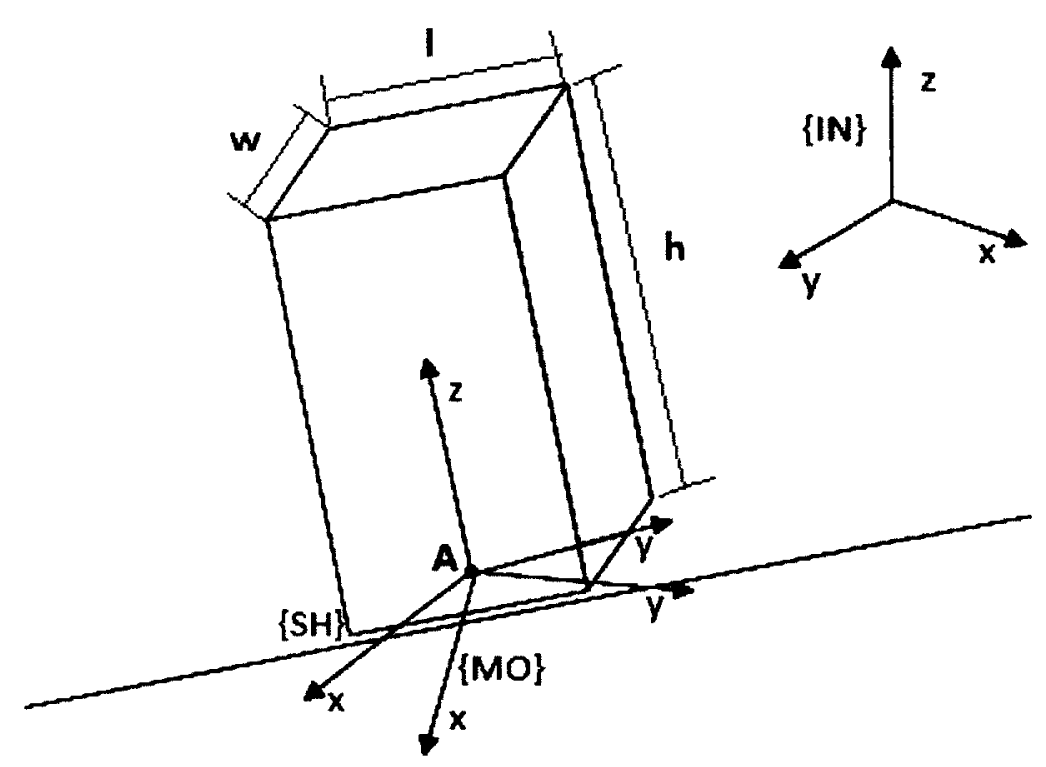

Figure 12: Coordinate systems used in the derivation of the three-dimensional Graham shipboard postural stability model.

\subsubsection{Kinematics of the Graham Model}

Defining the point of attachment of the model to the motion environment as point $A$, the position of the centre of gravity $(C G)$ of the block $\{r\}_{C G}$ can be written as

$$
\{r\}_{C G}^{I N}=\{r\}_{A}^{I N}+\{r\}_{C G / A}^{I N}
$$

where the superscript is the coordinate system in which the vector is defined and the subscript indicates the point(s) to which the vector applies. Defining two rotational transformation matrices from the model frame to the ship's coordinate system, $\left[T_{M O S H}\right]$, and then from the ship frame to the inertial frame, $\left[T_{S H I N}\right]$, the equation can be written as

$$
\{r\}_{C G}^{I N}=\{r\}_{A}^{I N}+\left[T_{S H I N}\right]\left[T_{M O S H}\right]\{r\}_{C G / A}^{M O}
$$


where for a rotation matrix $\mathrm{T}$ the first subscript denotes the coordinate system rotated from and the second subscript denotes the coordinate system rotated to. The rotation matrix from the ship to inertial frame is a local to global XYZ Euler angle rotation that depends on the ship orientation angles. The rotation matrix from the model to ship frame is defined as a single rotation about the $z$ axis in the ship frame,

$$
\boldsymbol{T}_{\text {MOSH }}=\left[\begin{array}{ccc}
\cos \beta & -\sin \beta & 0 \\
\sin \beta & \cos \beta & 0 \\
0 & 0 & 1
\end{array}\right]
$$

where $\beta$ is the constant angle between the ship and model frames.

Taking the derivative of the position vector, noting that the angle between the model and ship frames is constant, and that the height of the centre of gravity above point $A$ in the model frame is constant, the velocity of the centre of gravity in the inertial frame can be written as

$$
\{v\}_{C G}^{I N}=\{v\}_{A}^{I N}+\left[\dot{T}_{S H I N}\right]\left[T_{M O S H}\right]\{r\}_{C G / A}^{M O}
$$

Taking the time derivative of the velocity expression results in the acceleration expression

$$
\{a\}_{C G}^{I N}=\{a\}_{A}^{I N}+\left[\ddot{T}_{S H I N}\right]\left[T_{M O S H}\right]\{r\}_{C G / A}^{M O}
$$

Now that the acceleration of the body is known, the dynamic equations can be developed and solved in order to find the interface forces and moments at the attachment point $A$. 


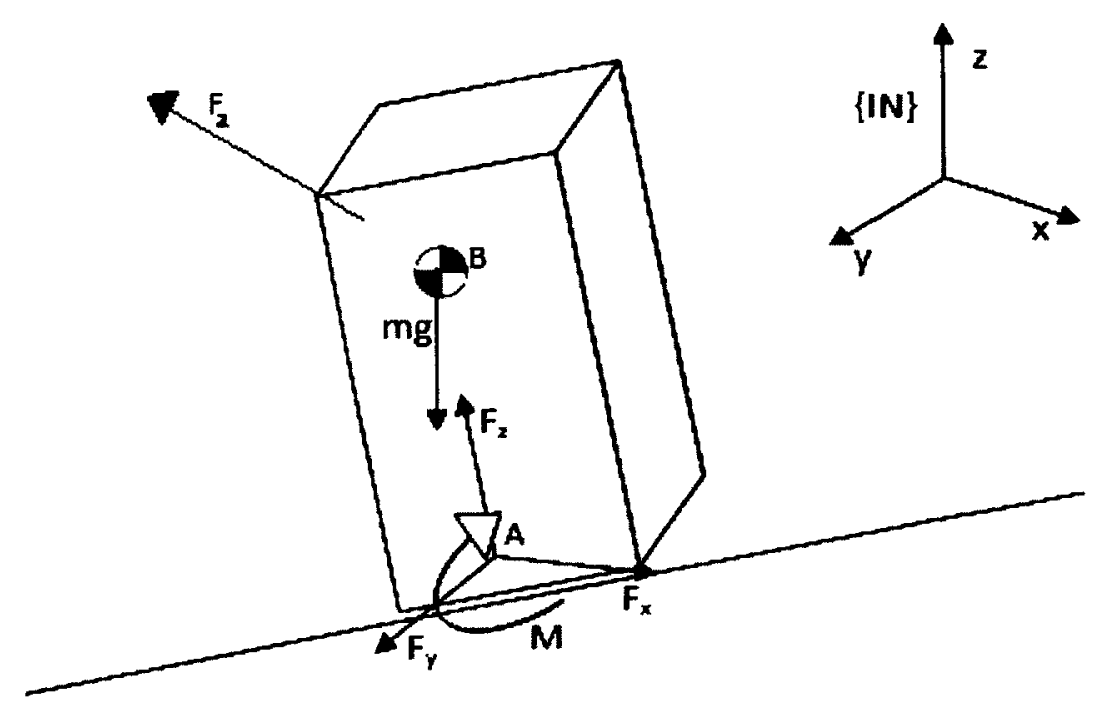

Figure 13: Forces acting on the three-dimensional Graham model.

\subsubsection{Dynamics of the Graham Model}

The forces acting on the body are the gravitational forces, inertial forces from the motion of the body, the reaction forces at the attachment point, and the externally applied unsteady load. If the attachment point is assumed to be a fixed point of attachment, the reaction forces and moments will be acting at that point. Figure 13 provides a diagram of the forces acting on the body.

Newton's law relates the forces acting on the block in the inertial frame to the acceleration of the centre of mass of the block such that

$$
\sum\{F\}^{I N}=m\{a\}_{C G}^{I N}
$$

where $m$ is the mass of the block. An expression for the acceleration of the centre of gravity was determined from the kinematic derivation and given in Equation 5. The applied forces, comprising the gravitational and reaction forces at the attachment 
point, are

$$
\sum\{F\}^{I N}=\left\{\begin{array}{c}
0 \\
0 \\
-m g
\end{array}\right\}^{I N}+\left\{\begin{array}{c}
F_{x} \\
F_{y} \\
F_{z}
\end{array}\right\}^{I N}+\left\{\begin{array}{c}
F_{A x} \\
F_{A y} \\
F_{A z}
\end{array}\right\}^{I N}
$$

where $g$ is the acceleration due to gravity; $F_{x}, F_{y}$, and $F_{z}$ are the components of the reaction force; $\{F\}_{A}$ is the unsteady externally-applied load. It is convenient for subsequent sliding and tipping index evaluation for the reaction forces to be expressed in the model frame; so the force summation then becomes,

$$
\sum\{F\}^{I N}=\left\{\begin{array}{c}
0 \\
0 \\
-m g
\end{array}\right\}^{I N}+\left[T_{S H I N}\right]\left[T_{M O S H}\right]\left\{\begin{array}{c}
F_{x} \\
F_{y} \\
F_{z}
\end{array}\right\}^{M O}+\left\{\begin{array}{c}
F_{A x} \\
F_{A y} \\
F_{A z}
\end{array}\right\}^{I N}
$$

Substituting Equation 5 and Equation 8 into Equation 6 and rearranging the resulting equation such that the unknowns are on the left hand side, the translational dynamic equation then becomes

$$
\left[T_{S H I N}\right]\left[T_{M O S H}\right]\left\{\begin{array}{c}
F_{x} \\
F_{y} \\
F_{z}
\end{array}\right\}^{M O}=\left\{\begin{array}{c}
0 \\
0 \\
m g
\end{array}\right\}^{I N}-\left\{\begin{array}{c}
F_{A x} \\
F_{A y} \\
F_{A z}
\end{array}\right\}^{I N}
$$

where all the variables on the right side are known from the ship motion and the position of the block on the ship deck. The externally applied force is calculated from 
an interface force model and will be known.

The generalized Euler equation for moments about the centre of gravity in the model frame is

$$
\sum\{M\}_{C G}^{M O}=\left[I_{C G}\right]\{\alpha\}+\{\omega\} \times\left[I_{C G}\right]\{\omega\}
$$

where $\{\alpha\}$ is the angular acceleration of the block, $\{\omega\}$ is the angular velocity of the block, and $\left[I_{C G}\right]$ is the mass moment of inertia of the block. Since the block is not moving relative to the ship deck, the angular velocity and acceleration are those of the ship which are considered to be perscribed functions of time. In practice, the ship motion may be obtained from sea trial or model test data, or alternatively simulated.

The moments acting on the block are the reaction moments at the attachment point, the moments caused by the deck reaction forces about the centre of gravity, and the moments caused by the externally-applied force about the centre of gravity. Moments about the centre of gravity are expressed as

$$
\sum\{M\}_{C G}^{M O}=\left\{\begin{array}{c}
M_{x} \\
M_{y} \\
M_{z}
\end{array}\right\}^{M O}+\{r\}_{A / C G}^{M O} \times\left\{\begin{array}{c}
F_{x} \\
F_{y} \\
F_{z}
\end{array}\right\}^{M O}+\{r\}_{B / C G}^{M O} \times\left\{\begin{array}{c}
F_{A x} \\
F_{A y} \\
F_{A z}
\end{array}\right\}^{M O}
$$

where $M_{x}, M_{y}$, and $M_{z}$ are the reaction moments at the attachment point. Equation 11 is substituted into Equation 10 and the resulting equation is rearranged to 
isolate the reaction moments

$$
\begin{aligned}
& \left\{\begin{array}{l}
M_{x} \\
M_{y} \\
M_{z}
\end{array}\right\}^{M O}=\left[I_{C G}\right]\{\alpha\}+\{\omega\} \times\left[I_{C G}\right]\{\omega\}-\{r\}_{A / C G}^{M O} \times\left\{\begin{array}{c}
F_{x} \\
F_{y} \\
F_{z}
\end{array}\right\}^{M O} \\
& -\{r\}_{B / C G}^{M O} \times\left[T_{S H M O}\right]\left[T_{I N S H}\right]\left\{\begin{array}{c}
F_{A x} \\
F_{A y} \\
F_{A z}
\end{array}\right\}^{I N}
\end{aligned}
$$

To obtain the reaction forces and moments, Equation 9 is first solved for the reaction forces; then Equation 12 is solved for the reaction moments.

\subsection{Unsteady Cart Load Model}

The load model used for the investigation of the effect of external shipboard loads is a cart model. The model was defined as a two mass system where one mass can translate freely along a prescribed axis, but not rotate; the second mass can rotate relative to the first mass but not translate relative to it. The following sections provide the mathematical derivation of the model using Newton-Euler equations.

\subsubsection{Model Definition and Coordinate Systems}

The two mass cart system is illustrated schematically in Figure 14. One mass, $m_{1}$, is attached to the deck of the ship, placed initially with the centre of mass aligned over point $A$, similar to the spatial Graham model. This mass has one degree of freedom allowing it to translate in the $x$ direction in the model coordinate frame. 
The second mass, $m_{2}$ is attached to $m_{1}$ at point $R$ in the model coordinate frame. The vector defining the location of this attachment point relative to the centre of mass of $m_{1}$, point $B$, is defined as $\left\{p_{R / B}\right\}$. The revolute joint between the lower and upper masses only allows one degree of freedom. The external force is applied to the cart model at an attachment point on $m_{2}$ where the location is defined by a vector from the centre of mass of $m_{2}$.

The coordinate systems used for the derivation can also be seen in Figure 14. The inertial frame and ship frame are similar to those defined for the spatial Graham model. The model frame $[\mathrm{MO}]$ is attached to the ship deck at point $A$ and the $x$ direction is in the same direction as the cart translation direction. Initially the cart is placed such that the centre of mass is above point $A$. The next coordinate system is called the top coordinate system, [TO]. This coordinate system is attached to $m_{2}$ at the point of rotation relative to $m_{1}$. Initially the coordinate systems are aligned such that the $x$ and $y$ directions of the coordinate systems coincide. The rotational transformation from the top coordinate system to the model frame is a rotation about the $z$ axis. This rotation angle is the angle between $m_{1}$ and $m_{2}$, and is not a constant value.

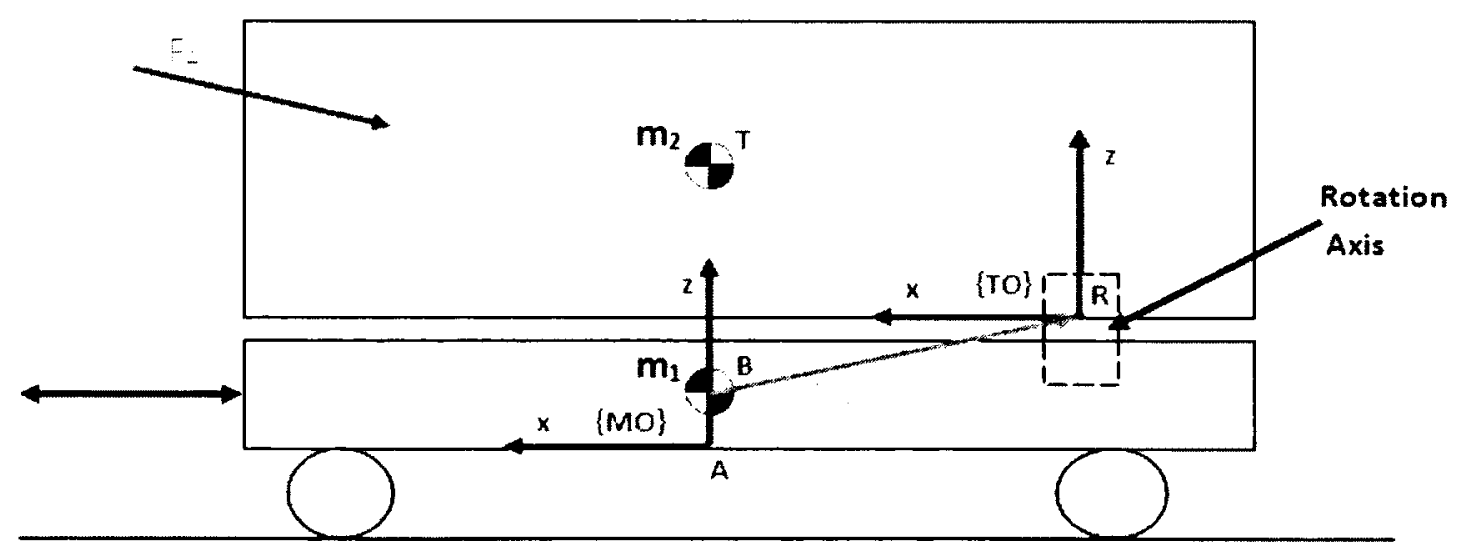

Figure 14: Coordinate systems used in the derivation of in-plane cart load model. 


\subsubsection{Kinematics for Two-body Cart}

\section{Translating Mass}

The position of the centre of mass of $m_{1}$, point $B$, in the inertial frame is defined relative to point $A$ as

$$
\{r\}_{B}^{I N}=\{r\}_{A}^{I N}+\{r\}_{B / A}^{I N}
$$

Since $m_{1}$ is only allowed to translate in the $x$ direction in the model frame, it is advantageous to define the position of the centre of mass with respect to point $A$ in the model frame. This can be done using two rotation matrices, one from the ship frame to the inertial frame, then from the model frame to the ship frame.

$$
\{r\}_{B}^{I N}=\{r\}_{A}^{I N}+\left[T_{S H I N}\right]\left[T_{M O S H}\right]\{r\}_{B / A}^{M O}
$$

To solve for the velocity at point $B$, the derivative of the position vector is found to be

$$
\{v\}_{B}^{I N}=\{v\}_{A}^{I N}+\left[\dot{T}_{S H I N}\right]\left[T_{M O S H}\right]\{r\}_{B / A}^{M O}+\left[T_{S H I N}\right]\left[T_{M O S H}\right]\{v\}_{B / A}^{M O}
$$

knowing that the rotation from the ship frame to the model frame is constant. The derivative of the rotation matrix from the inertial frame to the ship frame can be found using the known ship motions. Note that the velocity of point $B$ with respect to point $A$ is not constant, but the direction of the vector is known. Taking the derivative of the velocity vector results in the acceleration of point $B$.

$$
\begin{array}{r}
\{a\}_{B}^{I N}=\{a\}_{A}^{I N}+\left[\ddot{T}_{S H I N}\right]\left[T_{M O S H}\right]\{r\}_{B / A}^{M O}+2\left[\dot{T}_{S H I N}\right]\left[T_{M O S H}\right]\{v\}_{B / A}^{M O}+ \\
{\left[T_{S H I N}\right]\left[T_{M O S H}\right]\{a\}_{B / A}^{M O}}
\end{array}
$$


This is an expression of the acceleration of point $B$ in terms of known quantities. Next the kinematics for the rotating mass $m_{2}$ will be derived.

\section{Rotating Mass}

The point of attachment for $m_{2}$ is defined as point $R$. So, defining the centre of mass location as point $T$, the position vector can be written as

$$
\{r\}_{T}^{I N}=\{r\}_{R}^{I N}+\left[T_{S H I N}\right]\left[T_{M O S H}\right]\left[T_{T O M O}\right]\{r\}_{T / R}^{T O}
$$

noting that the relative position vector has been expressed in the top coordinate frame because it is a known vector in the top coordinate frame. The rotation from the model frame to the top frame is not constant and therefore the derivative of the rotation matrix must be found. The velocity of point $T$ can be found by taking the derivative of the position vector such that

$$
\begin{aligned}
\{v\}_{T}^{I N}=\{v\}_{R}^{I N}+\left[\dot{T}_{S H I N}\right]\left[T_{M O S H}\right]\left[T_{T O M O}\right]\{r\}_{T / R}^{T O}+ \\
{\left[T_{S H I N}\right]\left[T_{M O S H}\right]\left[\dot{T}_{T O M O}\right]\{r\}_{T / R}^{T O} }
\end{aligned}
$$

Taking the derivative of the velocity vector will result in the acceleration vector such that

$$
\begin{aligned}
& \{a\}_{T}^{I N}=\{a\}_{R}^{I N}+\left[\ddot{T}_{S H I N}\right]\left[T_{M O S H}\right]\left[T_{T O M O}\right]\{r\}_{T / R}^{T O}+ \\
& \quad 2\left[\dot{T}_{S H I N}\right]\left[T_{M O S H}\right]\left[\dot{T}_{T O M O}\right]\{r\}_{T / R}^{T O}+\left[T_{S H I N}\right]\left[T_{M O S H}\right]\left[\ddot{T}_{T O M O}\right]\{r\}_{T / R}^{T O}
\end{aligned}
$$

As can be seen from Equation 19, the acceleration of point $R$ must be known. This point is on the interface between $m_{1}$ and $m_{2}$ and thus the acceleration is the same at that point on each body. The acceleration of point $R$ can be found similarly to the 
acceleration of point $B$ on the translating mass. The acceleration of point $R$ in the inertial frame is given by

$$
\begin{aligned}
\{a\}_{R}^{I N}=\{a\}_{A}^{I N}+\left[\ddot{T}_{S H I N}\right]\left[T_{M O S H}\right]\{r\}_{R / A}^{M O}+ \\
2\left[\dot{T}_{S H I N}\right]\left[T_{M O S H}\right]\{v\}_{R / A}^{M O}+\left[T_{S H I N}\right]\left[T_{M O S H}\right]\{a\}_{R / A}^{M O}
\end{aligned}
$$

where again the vectors for point $R$ have been expressed in the model frame. This form of the acceleration is not desirable because it requires solving for the relative acceleration of point $R$ in the model frame as well. This can be simplified by considering the direction of the acceleration of $m_{1}$ in the model frame. Since the body is only allowed to translate in one direction and there is no rotation in the model frame, that means that at any point on the body the velocity and acceleration vectors are the same in the model frame. This means the equation can be written as

$$
\begin{aligned}
\{a\}_{R}^{I N}=\{a\}_{A}^{I N}+\left[\ddot{T}_{S H I N}\right]\left[T_{M O S H}\right]\{r\}_{R / A}^{M O}+ \\
2\left[\dot{T}_{S H I N}\right]\left[T_{M O S H}\right]\{v\}_{B / A}^{M O}+\left[T_{S H I N}\right]\left[T_{M O S H}\right]\{a\}_{B / A}^{M O}
\end{aligned}
$$

This still requires simplification because the position of point $R$ with respect to point $A$ is not known. This can, however, be defined as the sum of the position point $B$ with respect to point $A$ and the position of point $R$ with respect to point $B$ such that

$$
\begin{aligned}
\{a\}_{R}^{I N}=\{a\}_{A}^{I N}+\left[\ddot{T}_{S H I N}\right]\left[T_{M O S H}\right]\left(\{r\}_{B / A}^{M O}+\{r\}_{R / B}^{M O}\right)+ \\
2\left[\dot{T}_{S H I N}\right]\left[T_{M O S H}\right]\{v\}_{B / A}^{M O}+\left[T_{S H I N}\right]\left[T_{M O S H}\right]\{a\}_{B / A}^{M O}
\end{aligned}
$$


Expanding the equation results in

$$
\begin{array}{r}
\{a\}_{R}^{I N}=\{a\}_{A}^{I N}+\left[\ddot{T}_{S H I N}\right]\left[T_{M O S H}\right]\{r\}_{B / A}^{M O}+2\left[\dot{T}_{S H I N}\right]\left[T_{M O S H}\right]\{v\}_{B / A}^{M O}+ \\
{\left[T_{S H I N}\right]\left[T_{M O S H}\right]\{a\}_{B / A}^{M O}+\left[\ddot{T}_{S H I N}\right]\left[T_{M O S H}\right]\{r\}_{R / B}^{M O}}
\end{array}
$$

which is equal to

$$
\{a\}_{R}^{I N}=\{a\}_{B}^{I N}+\left[\ddot{T}_{S H I N}\right]\left[T_{M O S H}\right]\{r\}_{R / B}^{M O}
$$

This method can be verified by solving for the acceleration of point $R$ using another point of reference. The position of point $R$ could also be written as

$$
\{r\}_{R}^{I N}=\{r\}_{B}^{I N}+\left[T_{S H I N}\right]\left[T_{M O S H}\right]\{r\}_{R / B}^{M O}
$$

Knowing that the distance between points $B$ and $R$ is constant, because they are on the same body, taking the second derivative of the position vector will result in the acceleration vector,

$$
\{a\}_{R}^{I N}=\{a\}_{B}^{I N}+\left[\ddot{T}_{S H I N}\right]\left[T_{M O S H}\right]\{r\}_{R / B}^{M O}
$$

which is the same result as from the previous formulation.

The rotation matrix from the model frame to the top frame is defined by the rotation of $m_{2}$ with respect to $m_{1}$. The corresponding rotation angle is not known and must be solved from the equations, so it is required to rearrange the equation to include the angle. The rotation between the model and top frames is a single rotation 
about the $z$ axis which is written as

$$
\left[T_{\text {TOMO }}\right]=\left[\begin{array}{ccc}
\sin \theta & \cos \theta & 0 \\
\cos \theta & -\sin \theta & 0 \\
0 & 0 & 0
\end{array}\right]
$$

The first and second derivatives of the rotation matrix are required and can be written as

$$
\left[\dot{T}_{\text {TOMO }}\right]=\left[\begin{array}{ccc}
\dot{\theta} \cos \theta & -\dot{\theta} \sin \theta & 0 \\
-\dot{\theta} \sin \theta & -\dot{\theta} \cos \theta & 0 \\
0 & 0 & 0
\end{array}\right]
$$

and

$$
\left[\ddot{T}_{\text {TOMO }}\right]=\left[\begin{array}{ccc}
\ddot{\theta} \cos \theta-\dot{\theta}^{2} \sin \theta & -\ddot{\theta} \sin \theta-\dot{\theta}^{2} \cos \theta & 0 \\
-\ddot{\theta} \sin \theta-\dot{\theta}^{2} \cos \theta & -\ddot{\theta} \cos \theta+\dot{\theta}^{2} \sin \theta & 0 \\
0 & 0 & 0
\end{array}\right]
$$

The angular acceleration, which is one of the variables to be solved, is embedded in $\left[\ddot{T}_{\text {TOMO }}\right]$ and can be factored out of the matrix.

$$
\left[\ddot{T}_{\text {TOMO }}\right]=\ddot{\theta}\left[\begin{array}{ccc}
\cos \theta & -\sin \theta & 0 \\
-\sin \theta & -\cos \theta & 0 \\
0 & 0 & 0
\end{array}\right]+\left[\begin{array}{ccc}
-\dot{\theta}^{2} \sin \theta & -\dot{\theta}^{2} \cos \theta & 0 \\
-\dot{\theta}^{2} \cos \theta & \dot{\theta}^{2} \sin \theta & 0 \\
0 & 0 & 0
\end{array}\right]
$$




\subsubsection{Dynamics for Two-body Cart}

The dynamic formulation for the cart model uses Newton-Euler equations. Using these equations for the two bodies of the cart will result in a twelve equation second order system. The translating mass $m_{1}$ is free to move in the $x$ direction and therefore does not have a resisting force in that direction. This means that the acceleration in the $x$ direction in the model frame is an unknown to be solved. Similarly, the rotating mass $m_{2}$ is free to rotate about the $z$ axis in the model frame and therefore does not have a resistive moment in that direction. This however does introduce the angular acceleration as an unknown. The applied and reaction forces can be seen in the free body diagram in Figure 15.
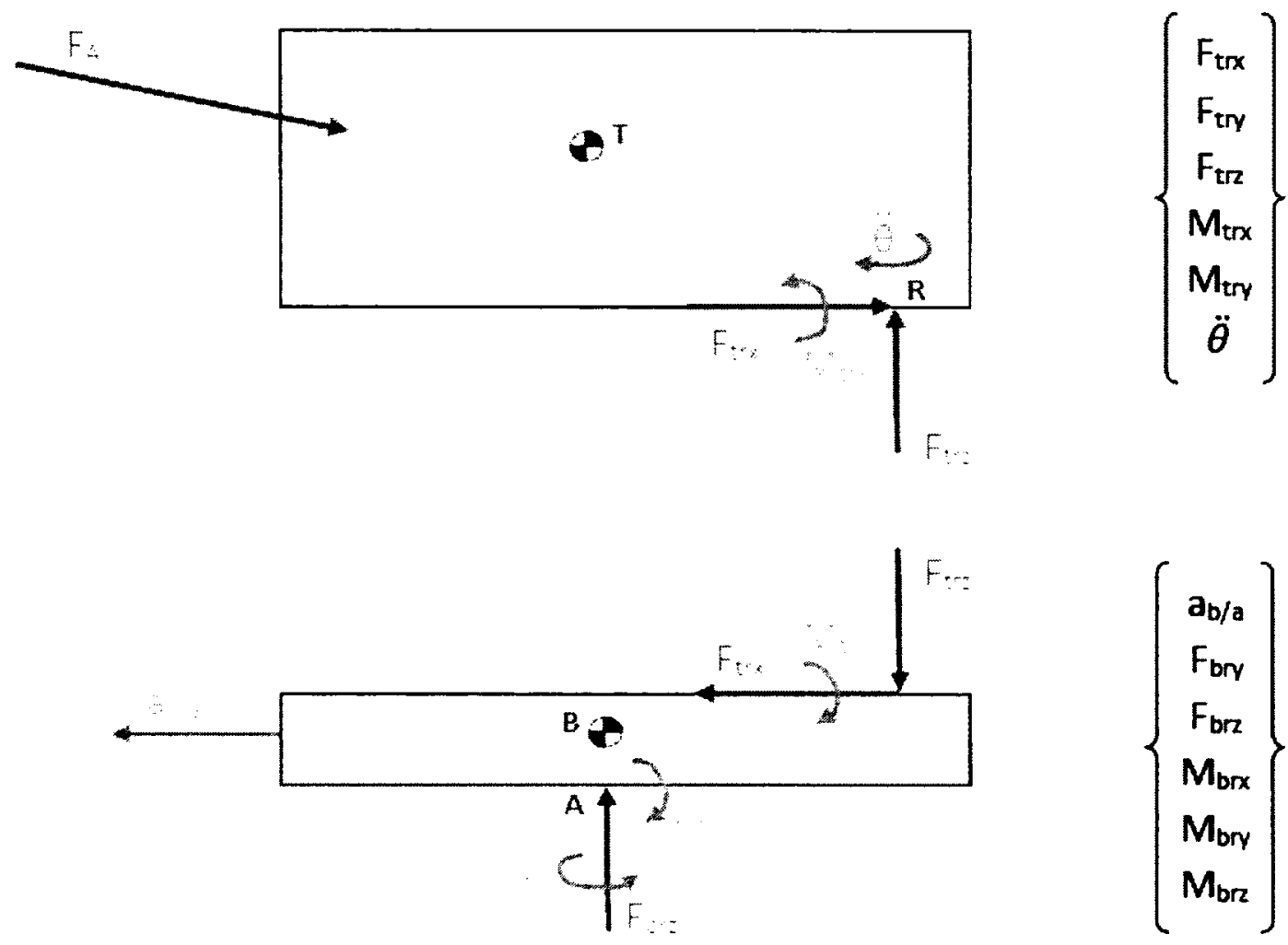

$\left\{\begin{array}{c}a_{b / a} \\ F_{b r y} \\ F_{b r z} \\ M_{b r x} \\ M_{b r y} \\ M_{b r z}\end{array}\right\}$

Figure 15: Free body diagram of the two cart masses with the state variables. 


\section{Translational Dynamics of the Translating Mass}

Starting with Newton's Law applied to $m_{1}$, the sum of the forces at $B$ is related to the acceleration at that point such that

$$
\sum\{F\}_{B}^{I N}=m_{1}\{a\}_{B}^{I N}
$$

From the free body diagram of $m_{1}$ it can be seen that the only external forces acting on it are the reaction forces from the ship deck and from $m_{2}$.

$$
\sum\{F\}_{B}^{I N}=\{F\}_{B R}^{I N}-\{F\}_{T R}^{I N}-m_{1}\{g\}^{I N}
$$

Substituting Equation 32 into Equation 31 results in

$$
\{F\}_{B R}^{I N}-\{F\}_{T R}^{I N}-m_{1}\{g\}^{I N}=m_{1}\{a\}_{B}^{I N}
$$

From the kinematic equations, the acceleration of point $B$ is solved in the inertial frame. Substituting the solution for the acceleration results in

$$
\begin{aligned}
\{F\}_{B R}^{I N}- & \{F\}_{T R}^{I N}=m_{1}\left[\{a\}_{A}^{I N}+\left[\ddot{T}_{S H I N}\right]\left[T_{M O S H}\right]\{r\}_{B / A}^{M O}+\right. \\
& \left.2\left[\dot{T}_{S H I N}\right]\left[T_{M O S H}\right]\{v\}_{B / A}^{M O}+\left[T_{S H I N}\right]\left[T_{M O S H}\right]\{a\}_{B / A}^{M O}\right]+m_{1}\{g\}^{I N}
\end{aligned}
$$

Placing the unknowns on the left hand side results in

$$
\begin{aligned}
\{F\}_{B R}^{I N}-\{F\}_{T R}^{I N}-m_{1}\left[T_{S H I N}\right]\left[T_{M O S H}\right]\{a\}_{B / A}^{M O}= & \\
m_{1}\left[\{a\}_{A}^{I N}+\left[\ddot{T}_{S H I N}\right]\left[T_{M O S H}\right]\{r\}_{B / A}^{M O}+2\left[\dot{T}_{S H I N}\right]\left[T_{M O S H}\right]\{v\}_{B / A}^{M O}\right]+ & m_{1}\{g\}^{I N}
\end{aligned}
$$


It is required to solve for the reaction forces in the model frame because $F_{B R x}^{M O}$ is known to be zero because of the free translation in the $\mathrm{x}$ direction in the model frame. This will then allow for the system to be solved using the twelve available equations.

$$
\begin{aligned}
{\left[T_{S H I N}\right]\left[T_{M O S H}\right]\{F\}_{B R}^{M O}-\left[T_{S H I N}\right]\left[T_{M O S H}\right]\{F\}_{T R}^{M O}-} \\
m_{1}\left[T_{S H I N}\right]\left[T_{M O S H}\right]\{a\}_{B / A}^{M O}=m_{1}\left[\{a\}_{A}^{I N}+\left[\ddot{T}_{S H I N}\right]\left[T_{M O S H}\right]\{r\}_{B / A}^{M O}+\right. \\
\left.2\left[\dot{T}_{S H I N}\right]\left[T_{M O S H}\right]\{v\}_{B / A}^{M O}\right]+m_{1}\{g\}^{I N}
\end{aligned}
$$

where

$$
\{a\}_{B / A}^{M O}=\left[\begin{array}{c}
a_{B / A}^{M O} \\
0 \\
0
\end{array}\right]
$$

and

$$
\{F\}_{B R}^{M O}=\left[\begin{array}{c}
0 \\
F_{B R y}^{M O} \\
F_{B R z}^{M O}
\end{array}\right]
$$


Writing Equation 36 in terms of the state variables results in

$$
\begin{aligned}
& {\left[\left[T_{S H I N}\right]\left[T_{M O S H}\right]-\left[T_{S H I N}\right]\left[T_{M O S H}\right]\right]\left\{\begin{array}{c}
-m_{1} a_{B / A}^{M O} \\
F_{B R y}^{M O} \\
F_{B R z}^{M O} \\
F_{T R x}^{M O} \\
F_{T R y}^{M O} \\
F_{T R z}^{M O}
\end{array}\right\}} \\
& =m_{1}\left[\{a\}_{A}^{I N}+\left[\ddot{T}_{S H I N}\right]\left[T_{M O S H}\right]\{r\}_{B / A}^{M O}+2\left[\dot{T}_{S H I N}\right]\left[T_{M O S H}\right]\{v\}_{B / A}^{M O}\right]+ \\
& m_{1}\{g\}^{I N}
\end{aligned}
$$

\section{Rotational Dynamics of the Translating Mass}

This derivation begins with Euler's moment equation in the model coordinate frame with the moments taken about the centre of mass of $m_{1}$

$$
\sum\{M\}_{B}^{M O}=\left[I_{B_{C G}}\right]\{\alpha\}_{B}^{M O}+\{\omega\}_{B}^{M O} \times\left[I_{B_{C G}}\right]\{\omega\}_{B}^{M O}
$$

where $\left\{\omega_{B}^{M O}\right\}$ and $\left\{\alpha_{B}^{M O}\right\}$ are the angular velocity and acceleration of the model coordinate frame. These are known from the ship motions. The external moments being applied on $m_{1}$ are

$$
\sum\{M\}_{B}^{M O}=\{M\}_{B R}^{M O}-\{M\}_{T R}^{M O}+\{r\}_{R / B}^{M O} \times\{F\}_{T R}^{M O}+\{r\}_{A / B}^{M O} \times\{F\}_{B R}^{M O}
$$


Substituting Equation 40 into Equation 41 and moving all the unknowns to the left hand side will result in

$$
\begin{aligned}
\{M\}_{B R}^{M O}-\{M\}_{T R}^{M O}+\{r\}_{R / B}^{M O} \times\{F\}_{T R}^{M O}+\{r\}_{A / B}^{M O} \times\{F\}_{B R}^{M O}= & \\
& {\left[I_{B}\right]\{\alpha\}_{A}^{M O}+\{\omega\}_{A}^{M O} \times\left[I_{B}\right]\{\omega\}_{A}^{M O} }
\end{aligned}
$$

Where $\alpha_{B}^{M O}=\alpha_{A}^{M O}$ and $\omega_{B}^{M O}=\omega_{A}^{M O}$ because the bottom mass is a rigid body and is not rotating about any of the axis in the model frame.

In order to represent Equation 42 in terms of the state variables, the cross products can be expanded to

$$
\begin{array}{r}
\{M\}_{B R}^{M O}-\{M\}_{T R}^{M O}+\left[\begin{array}{c}
r_{R / B y}^{M O} F_{T R z}^{M O}-r_{R / B z}^{M O} F_{T R y}^{M O} \\
r_{R / B z}^{M O} F_{T R x}^{M O}-r_{R / B x}^{M O} F_{T R z}^{M O} \\
r_{R / B x}^{M O} F_{T R y}^{M O}-r_{R / B y}^{M O} F_{T R x}^{M O}
\end{array}\right]+\left[\begin{array}{c}
r_{A / B y}^{M O} F_{B R z}^{M O}-r_{A / B z}^{M O} F_{B R y}^{M O} \\
r_{A / B z}^{M O} F_{B R x}^{M O}-r_{A / B x}^{M O} F_{B R z}^{M O} \\
r_{A / B x}^{M O} F_{B R y}^{M O}-r_{A / B y}^{M O} F_{B R x}^{M O}
\end{array}\right] \\
=\left[I_{B_{C G}}\right]\{\alpha\}_{A}^{M O}+\{\omega\}_{A}^{M O} \times\left[I_{B_{C G}}\right]\{\omega\}_{A}^{M O}
\end{array}
$$

Remembering that $F_{B R x}^{M O}$ and $M_{T R z}^{T O}$ are equal to zero, and writing Equation 43 with expanded vectors produces

$$
\begin{gathered}
{\left[\begin{array}{c}
M_{B x}^{M O} \\
M_{B y}^{M O} \\
M_{B z}^{M O}
\end{array}\right]-\left[T_{T O M O}\right]\left[\begin{array}{c}
M_{T x}^{T O} \\
M_{T y}^{T O} \\
0
\end{array}\right]+\left[\begin{array}{c}
r_{R / B y}^{M O} F_{T R z}^{M O}-r_{R / B z}^{M O} F_{T R y}^{M O} \\
r_{R / B z}^{M O} F_{T R x}^{M O}-r_{R / B x}^{M O} F_{T R z}^{M O} \\
r_{R / B x}^{M O} F_{T R y}^{M O}-r_{R / B y}^{M O} F_{T R x}^{M O}
\end{array}\right]+\left[\begin{array}{c}
r_{A / B y}^{M O} F_{B R z}^{M O}-r_{A / B z}^{M O} F_{B R y}^{M O} \\
-r_{A / B x}^{M O} F_{B R z}^{M O} \\
r_{A / B x}^{M O} F_{B R y}^{M O}
\end{array}\right]} \\
=\left[I_{B C G}\right]\{\alpha\}_{A}^{M O}+\{\omega\}_{A}^{M O} \times\left[I_{B_{C G}}\right]\{\omega\}_{A}^{M O}
\end{gathered}
$$


Writing this in terms of the state variables produces

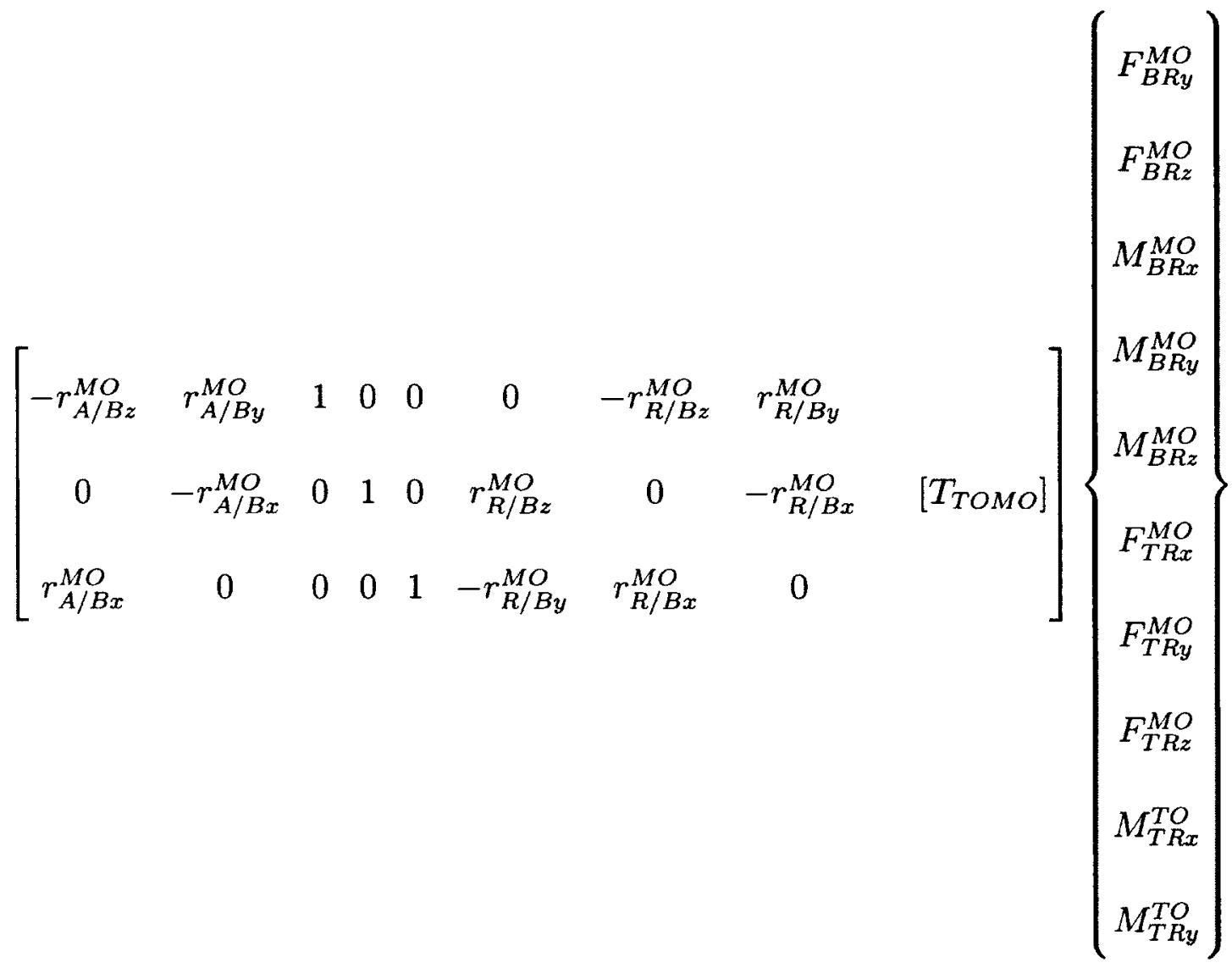

$$
\begin{aligned}
& =\left[I_{B_{C G}}\right]\{\alpha\}_{A}^{M O}+\{\omega\}_{A}^{M O} \times\left[I_{B_{C G}}\right]\{\omega\}_{A}^{M O}
\end{aligned}
$$

\section{Translational Dynamics of the Rotating Mass}

Starting from Newton's equation applied to $m_{2}$, the sum of the forces acting on $m_{2}$ in the inertial frame are

$$
\sum\{F\}^{I N}=m_{2}\{a\}_{T}^{I N}
$$

From the free body diagram (Figure 15) it is seen that the only external forces 
are the reaction forces at point $R$ and the applied external force $\{F\}_{A}$ so that

$$
\sum\{F\}^{I N}=\{F\}_{T R}^{I N}+\{F\}_{A}^{I N}+m_{2}\{g\}^{I N}
$$

Substituting Equation 47 into Equation 46 results in

$$
m_{2}\{a\}_{T}^{I N}=\{F\}_{T R}^{I N}+\{F\}_{A}^{I N}+m_{2}\{g\}^{I N}
$$

From the kinematic solution, the acceleration of point $T$ was solved in the inertial frame. Substituting the kinematic solution results in

$$
\begin{array}{r}
m_{2}\left[\{a\}_{R}^{I N}+\left[\ddot{T}_{\text {SHIN }}\right]\left[T_{M O S H}\right]\left[T_{T O M O}\right]\{r\}_{T / R}^{T O}+\right. \\
\left.2\left[\dot{T}_{S H I N}\right]\left[T_{M O S H}\right]\left[\dot{T}_{T O M O}\right]\{r\}_{T / R}^{T O}+\left[T_{S H I N}\right]\left[T_{M O S H}\right]\left[\ddot{T}_{T O M O}\right]\{r\}_{T / R}^{T O}\right]= \\
\{F\}_{T R}^{I N}+\{F\}_{A}^{I N}+m_{2}\{g\}^{I N}
\end{array}
$$

In Equation 49, the second derivative of the rotation matrix from the top frame to the model frame, $\left[\ddot{T}_{T O M O}\right]$, is not known. Substituting the expression for $\left[\ddot{T}_{\text {TOMO}}\right]$ results in

$$
\begin{gathered}
m_{2}\left[\{a\}_{R}^{I N}+\left[\ddot{T}_{S H I N}\right]\left[T_{M O S H}\right]\left[T_{T O M O}\right]\{r\}_{T / R}^{T O}+2\left[\dot{T}_{S H I N}\right]\left[T_{M O S H}\right]\left[\dot{T}_{T O M O}\right]\{r\}_{T / R}^{T O}\right. \\
+\left[T_{S H I N}\right]\left[T_{M O S H}\right]\left(\ddot{\theta}\left[\begin{array}{ccc}
\cos \theta & -\sin \theta & 0 \\
-\sin \theta & -\cos \theta & 0 \\
0 & 0 & 0
\end{array}\right]+\left[\begin{array}{ccc}
-\dot{\theta}^{2} \sin \theta & -\dot{\theta}^{2} \cos \theta & 0 \\
-\dot{\theta}^{2} \cos \theta & -\dot{\theta}^{2} \sin \theta & 0 \\
0 & 0 & 0
\end{array}\right]\right)\{r\}_{T / R}^{T O} \\
0
\end{gathered}
$$


Expanding the equation produces

$$
\begin{gathered}
m_{2}\left[\{a\}_{R}^{I N}+\left[\ddot{T}_{S H I N}\right]\left[T_{M O S H}\right]\left[T_{T O M O}\right]\{r\}_{T / R}^{T O}+2\left[\dot{T}_{S H I N}\right]\left[T_{M O S H}\right]\left[\dot{T}_{T O M O}\right]\{r\}_{T / R}^{T O}\right. \\
+\left[T_{S H I N}\right]\left[T_{M O S H}\right]\left(\ddot{\theta}\left[\begin{array}{c}
r_{T / R x}^{T O} \cos \theta-r_{T / R y}^{T O} \sin \theta \\
-r_{T / R x}^{T O} \sin \theta-r_{T / R y}^{T O} \cos \theta
\end{array}\right]\right. \\
0 \\
\left.+\left[\begin{array}{c}
-r_{T / R x}^{T O} \dot{\theta}^{2} \sin \theta-r_{T / R y}^{T O} \dot{\theta}^{2} \cos \theta \\
-r_{T / R x}^{T O} \dot{\theta}^{2} \cos \theta-r_{T / R y}^{T O} \dot{\theta}^{2} \sin \theta \\
0
\end{array}\right]\right)=\{F\}_{T R}^{I N}+\{F\}_{A}^{I N}+m_{2}\{g\}^{I N}
\end{gathered}
$$

Defining

$$
\{A\}=\left[\begin{array}{c}
r_{T / R x}^{T O} \cos \theta-r_{T / R y}^{T O} \sin \theta \\
-r_{T / R x}^{T O} \sin \theta-r_{T / R y}^{T O} \cos \theta \\
0
\end{array}\right] \text { and }\{\mathrm{B}\}=\left[\begin{array}{c}
-r_{T / R x}^{T O} \dot{\theta}^{2} \sin \theta-r_{T / R y}^{T O} \dot{\theta}^{2} \cos \theta \\
-r_{T / R x}^{T O} \dot{\theta}^{2} \cos \theta-r_{T / R y}^{T O} \dot{\theta}^{2} \sin \theta \\
0
\end{array}\right]
$$

and rewriting Equation 51 leads to

$$
\begin{aligned}
m_{2}\left[\{a\}_{R}^{I N}+\left[\ddot{T}_{S H I N}\right]\left[T_{M O S H}\right]\left[T_{T O M O}\right]\{p\}_{T / R}^{T O}+\right. \\
2\left[\dot{T}_{S H I N}\right]\left[T_{M O S H}\right]\left[\dot{T}_{T O M O}\right]\{r\}_{T / R}^{T O}+\left[T_{S H I N}\right]\left[T_{M O S H}\right]\{B\}+ \\
{\left.\left[T_{S H I N}\right]\left[T_{M O S H}\right]\{A\} \ddot{\theta}\right]=\{F\}_{T R}^{I N}+\{F\}_{A}^{I N}-m_{2}\{g\}^{I N} }
\end{aligned}
$$


Substituting the expression for $\{a\}_{R}^{I N}$ from Equation 23 results in

$$
\begin{gathered}
m_{2}\left[\{a\}_{A}^{I N}+\left[\ddot{T}_{S H I N}\right]\left[T_{M O S H}\right]\{r\}_{B / A}^{M O}+2\left[\dot{T}_{S H I N}\right]\left[T_{M O S H}\right]\{v\}_{B / A}^{M O}+\right. \\
{\left[T_{S H I N}\right]\left[T_{M O S H}\right]\{a\}_{B / A}^{M O}+\left[\ddot{T}_{S H I N}\right]\left[T_{M O S H}\right]\{r\}_{R / B}^{M O}+} \\
{\left[\ddot{T}_{S H I N}\right]\left[T_{M O S H}\right]\left[T_{T O M O}\right]\{r\}_{T / R}^{T O}+2\left[\dot{T}_{S H I N}\right]\left[T_{M O S H}\right]\left[\dot{T}_{T O M O}\right]\{p\}_{T / R}^{T O}+} \\
\left.\left[T_{S H I N}\right]\left[T_{M O S H}\right]\{B\}+\left[T_{S H I N}\right]\left[T_{M O S H}\right]\{A\} \ddot{\theta}\right] \\
=\{F\}_{T R}^{I N}+\{F\}_{A}^{I N}-m_{2}\{g\}^{I N}
\end{gathered}
$$

Rearranging so the unknowns are on the left hand side yields

$$
\begin{array}{r}
{\left[T_{S H I N}\right]\left[T_{M O S H}\right]\{F\}_{T R}^{M O}-m_{2}\left[T_{S H I N}\right]\left[T_{M O S H}\right]\{a\}_{B / A}^{M O}-} \\
m_{2}\left[T_{S H I N}\right]\left[T_{M O S H}\right]\{A\} \ddot{\theta}=m_{2}\left[\{a\}_{A}^{I N}+\left[\ddot{T}_{S H I N}\right]\left[T_{M O S H}\right]\{r\}_{B / A}^{M O}+\right. \\
2\left[\dot{T}_{S H I N}\right]\left[T_{M O S H}\right]\{v\}_{B / A}^{M O}+\left[\ddot{T}_{S H I N}\right]\left[T_{M O S H}\right]\{r\}_{R / B}^{M O} \\
+\left[\ddot{T}_{S H I N}\right]\left[T_{M O S H}\right]\left[T_{T O M O}\right]\{r\}_{T / R}^{T O}+2\left[\dot{T}_{S H I N}\right]\left[T_{M O S H}\right]\left[\dot{T}_{T O M O}\right]\{r\}_{T / R}^{T O} \\
\left.+\left[T_{S H I N}\right]\left[T_{M O S H}\right]\{B\}\right]-\{F\}_{A}^{I N}-m_{2}\{g\}^{I N}
\end{array}
$$


Writing Equation 55 in terms of the state variables results in

$$
\begin{aligned}
& {\left[\begin{array}{lll}
-m_{2}\left[T_{S H I N}\right]\left[T_{M O S H}\right]\{A\} & {\left[T_{S H I N}\right]\left[T_{M O S H}\right]} & -m_{2}\left[T_{S H I N}\right]\left[T_{M O S H}\right]
\end{array}\right]\left\{\begin{array}{c}
\ddot{\theta} \\
F_{T R x}^{M O} \\
F_{T R y}^{M O} \\
F_{T R z}^{M O} \\
a_{B / A}^{M O}
\end{array}\right\}} \\
& =m_{2}\left[\{a\}_{A}^{I N}+\left[\ddot{T}_{S H I N}\right]\left[T_{M O S H}\right]\{r\}_{B / A}^{M O}+2\left[\dot{T}_{S H I N}\right]\left[T_{M O S H}\right]\{v\}_{B / A}^{M O}+\right. \\
& {\left[\ddot{T}_{S H I N}\right]\left[T_{M O S H}\right]\{r\}_{R / B}^{M O}+\left[\ddot{T}_{S H I N}\right]\left[T_{M O S H}\right]\left[T_{T O M O}\right]\{r\}_{T / R}^{T O}+} \\
& 2\left[\dot{T}_{S H I N}\right]\left[T_{M O S H}\right]\left[\dot{T}_{T O M O}\right]\{r\}_{T / R}^{T O} \\
& \left.+\left[T_{S H I N}\right]\left[T_{M O S H}\right]\{B\}\right]-\{F\}_{A}^{I N}-m_{2}\{g\}^{I N}
\end{aligned}
$$

\section{Rotational Dynamics of the Rotating Mass}

This portion of the derivation begins with Euler's moment equation in the top coordinate frame with the moments taken about the centre of mass of $m_{2}$ (Point T)

$$
\sum\{M\}_{T}^{T O}=\left[I_{T_{C G}}\right]\{\alpha\}_{T}^{T O}+\{\omega\}_{T}^{T O} \times\left[I_{T_{C G}}\right]\{\omega\}_{T}^{T O}
$$

From the free body diagram (Figure 15) the only external moments are the reaction moments, the moments caused by the reaction forces at point $R$, and the moment caused by the applied external force $\{F\}_{A}$ such that

$$
\sum\{M\}_{T}^{T O}=\{r\}_{F_{A}}^{T O} \times\{F\}_{A}^{T O}+\{M\}_{T R}^{T O}+\{r\}_{T R}^{T O} \times\{F\}_{T R}^{T O}
$$


The applied moment is known in the inertial frame, and the solution for the reaction forces is in the model frame. Transforming the forces into the top coordinate frame allows the expression to be written as

$$
\begin{array}{r}
\sum\{M\}_{T}^{T O}=\{r\}_{F_{A}}^{T O} \times\left[T_{M O T O}\right]\left[T_{S H M O}\right]\left[T_{I N S H}\right]\{F\}_{A}^{I N}+\left[T_{M O T O}\right]\{M\}_{T R}^{M O}+ \\
\{r\}_{T R}^{T O} \times\left[T_{M O T O}\right]\{F\}_{T R}^{M O}
\end{array}
$$

Substituting Equation 59 into Equation 57 results in

$$
\begin{aligned}
\{r\}_{F_{A}}^{T O} \times\left[T_{M O T O}\right]\left[T_{S H M O}\right]\left[T_{I N S H}\right]\{F\}_{A}^{I N}+\left[T_{T O M O}\right]\{M\}_{T R}^{M O}+ \\
\{r\}_{T R}^{T O} \times\left[T_{M O T O}\right]\{F\}_{T R}^{M O}=\left[I_{T_{C G}}\right]\{\alpha\}_{T}^{T O}+\{\omega\}_{T}^{T O} \times\left[I_{T_{C G}}\right]\{\omega\}_{T}^{T O}
\end{aligned}
$$

Since rotation of the top coordinate frame is known with respect to the model axes, the total rotation of the top mass and the derivatives can be written as

$$
\begin{gathered}
\{\theta\}_{T}^{T O}=\{\theta\}_{B}^{T O}+\{\theta\}_{T / B}^{T O} \\
\{\omega\}_{T}^{T O}=\{\omega\}_{B}^{T O}+\{\omega\}_{T / B}^{T O} \\
\{\alpha\}_{T}^{T O}=\{\alpha\}_{B}^{T O}+\{\alpha\}_{T / B}^{T O}
\end{gathered}
$$

where,

$$
\{\omega\}_{T / B}^{T O}=\left[\begin{array}{c}
0 \\
0 \\
\dot{\theta}
\end{array}\right] \text { and }\{\alpha\}_{\mathrm{T} / \mathrm{B}}^{\mathrm{TO}}=\left[\begin{array}{c}
0 \\
0 \\
\ddot{\theta}
\end{array}\right]
$$


Substituting these expressions into Equation 60 results in

$$
\begin{aligned}
\{r\}_{F_{A}}^{T O} \times\left[T_{M O T O}\right]\left[T_{S H M O}\right]\left[T_{I N S H}\right]\{F\}_{A}^{I N}+\left[T_{T O M O}\right]\{M\}_{T R}^{M O}+ \\
\{r\}_{T R}^{T O} \times\left[T_{T O M O}\right]\{F\}_{T R}^{M O}=\left[I_{T_{C G}}\right]\left(\{\alpha\}_{B}^{T O}+\{\alpha\}_{T / B}^{T O}\right)+ \\
\left(\{\omega\}_{B}^{T O}+\{\omega\}_{T / B}^{T O}\right) \times\left[I_{T_{C G}}\right]\left(\{\omega\}_{B}^{T O}+\{\omega\}_{T / B}^{T O}\right)
\end{aligned}
$$

Rearranging Equation 65 such that the unknowns are on the left hand side yields

$$
\begin{gathered}
{\left[\begin{array}{c}
M_{T R x}^{T O} \\
M_{T R y}^{T O} \\
0
\end{array}\right]+\{r\}_{T R}^{T O} \times\left[T_{M O T O}\right]\{F\}_{T R}^{M O}-\left[I_{T_{C G}}\right]\left[\begin{array}{c}
0 \\
0 \\
\ddot{\theta}
\end{array}\right]} \\
=\left(\{\omega\}_{B}^{T O}+\{\omega\}_{T / B}^{T O}\right) \times\left[I_{T_{C G}}\right]\left(\{\omega\}_{B}^{T O}+\{\omega\}_{T / B}^{T O}\right)+\left[I_{T_{C G}}\right]\{\alpha\}_{B}^{T O}+ \\
\{r\}_{F_{A}}^{T O} \times\left[T_{M O T O}\right]\left[T_{S H M O}\right]\left[T_{I N S H}\right]\{F\}_{A}^{I N}
\end{gathered}
$$

The cart dynamic solution is obtained by solving Equations 39, 45, 56, and 66 simultaneously. Combined, twelve equations are available to solve for the twelve unknown state variables.

\subsubsection{Interface Force}

Figure 16 shows schematic representations of a postural model (the expanded Graham model or the inverted pendulum model) interacting with a typical unsteady shipboard load (either a pendulating load or a co-planar cart load). In both cases, the postural model is attached to the unsteady load by the directed element shown schematically in Figure 17.

The directed element consists of a tension/compression force-producing member 

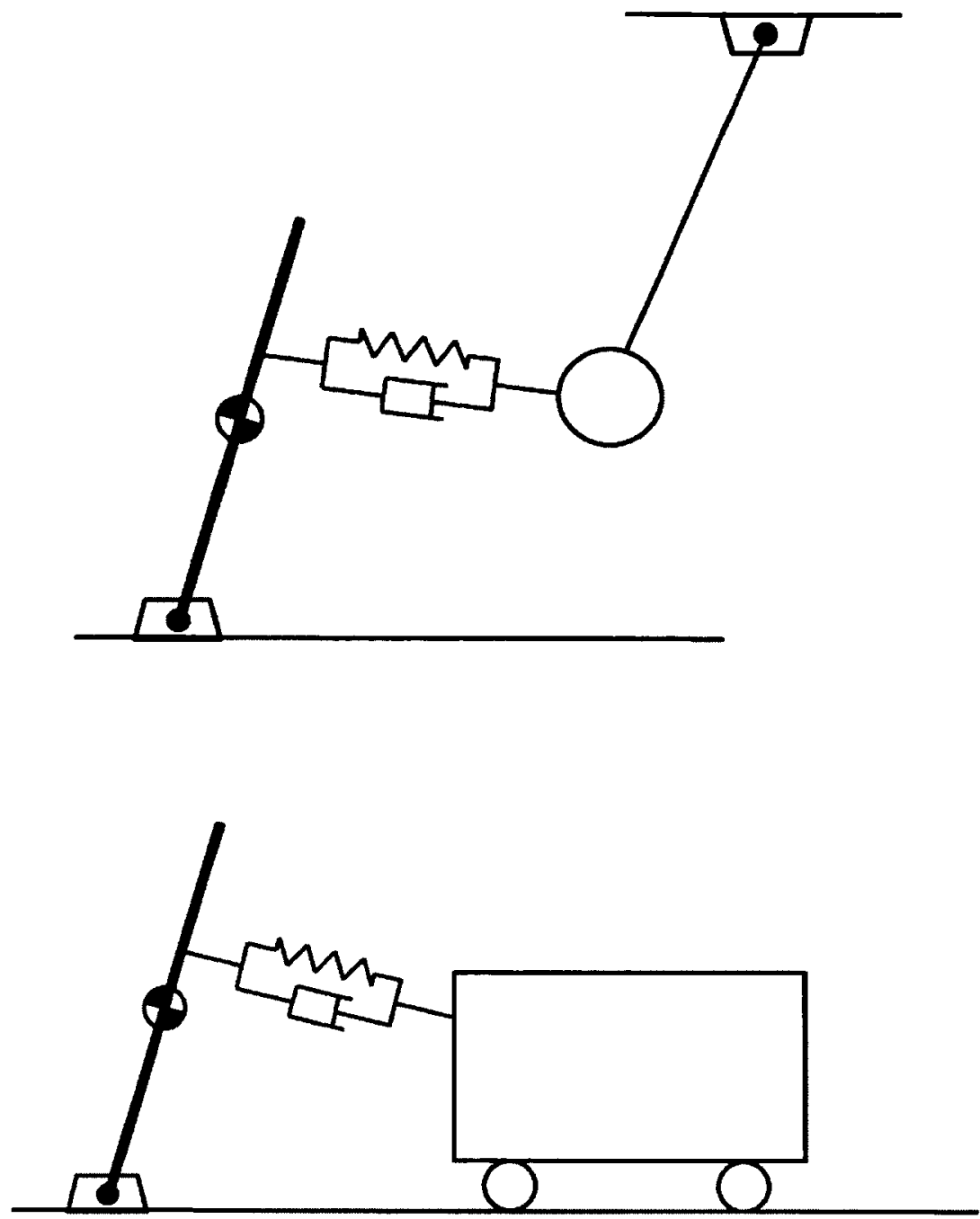

Figure 16: Schematic representation of postural stability models interacting with the pendulating load (top) and cart load (bottom).

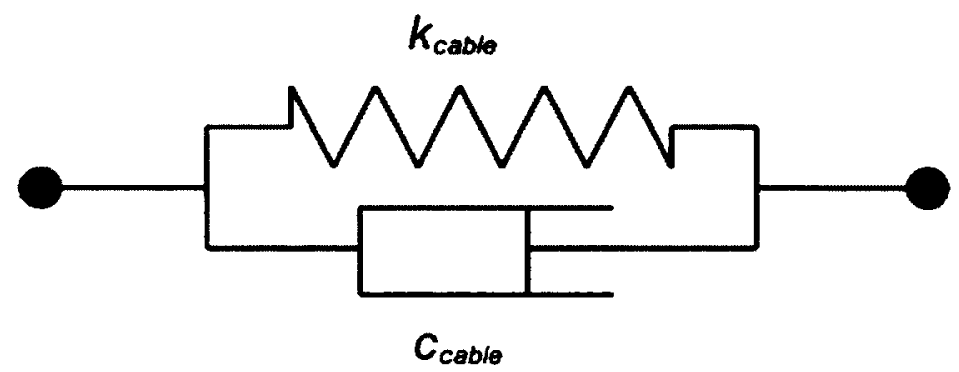

Figure 17: Directed element model. 
that can generate forces that depend upon the relative position and relative velocity between its two attachment points as well as some nonlinear behaviours. As implemented, the directed element has distinct linear stiffness properties in the tension and compression directions; distinct viscous damping properties in the tension and compression directions; as well as options for tension-only, compression-only, and no transmitted force behaviours to represent tension lines/cables, unattached push poles, and loss of contact situations, respectively.

\subsection{Stand-alone Computer Implementation}

The mathematical models are implemented in two simulation environments. The first environment is a Fortran monolithic simulation. The second is a distributed simulation environment running separate executables for each of the different models. This section will explain the Fortran simulation environment.

The solutions for the dynamics of the stability and load models as well as the coupling force have been implemented in separate Fortran subroutines. Each subroutine solves the system of equations for the unknowns. The main simulation executable, named DEPSM, controls the inputs, such as the perscribed ship motion, and then calls the required subroutines. Each subroutine has a required input file that will be discussed. The inverted pendulum model presented by Langlois [44] is implemented in this simulation environment and can be used with the models described in the previous sections such that the inverted pendulum stability model can be used with the cart load model.

There are required inputs for the main executable DEPSM that are provided via an ASCII input file. The input file provides data on the length of the simulation, the incremental time step, type of motion selected, as well as selection of which stability and load models will be used in the simulation. The length of the simulation 
Table 2: NATO Sea State Numerical Table for the Open Ocean North Atlantic

\begin{tabular}{|c|c|c|c|}
\hline \multirow[b]{2}{*}{ Sea State Number } & \multicolumn{2}{|c|}{ Significant Wave Height (m) } & \multirow{2}{*}{$\begin{array}{c}\text { Modal Wave Period (sec) } \\
\text { Most Probable }\end{array}$} \\
\hline & Range & Mean & \\
\hline $0-1$ & $0-0.1$ & 0.05 & - \\
\hline 2 & $0.1-0.5$ & 0.3 & 7.5 \\
\hline 3 & $0.5-1.25$ & 0.88 & 7.5 \\
\hline 4 & $1.25-2.5$ & 1.88 & 8.8 \\
\hline 5 & $2.5-4$ & 3.25 & 9.7 \\
\hline 6 & $4-6$ & 5 & 12.4 \\
\hline 7 & $6-9$ & 7.5 & 15.0 \\
\hline
\end{tabular}

and the time step are defined for the DEPSM function and passed to the stability and load model functions. This environment allows for several different excitation motions. There is an option for representative ship motion based on the sea state, ship speed, and ship heading; prescribed motions from an input file; as well as no motion at all. Sea state is a standardization developed by NATO in 1983 [49]. The sea conditions represented by each sea state can be seen in Table 2 . These motions are provided to the stability and load functions and are relative to the centre of mass of the ship. These motions are converted in the stability or load model functions to be defined at the attachment point of the model on the deck. There is also the ability to select the required stability and load models for simulation. This allows for the selection between the models presented here and the inverted pendulum stability model presented by Langlois [44] or a pendulating load model developed as part of the broader PSM project.

The input files for the stability and load models require information on their geometric and inertial properties. There are certain length vectors that need to be known. For example, the position of the centre of mass relative to the attachment 
point $A$ is needed for the Graham model. For the cart model there are additional lengths needed, such as the distance from the centre of mass $m_{1}$ to the articulation point of mass $m_{2}$, as well as the vector from the centre of mass $m_{2}$ to the location of the applied external force. One of the important input parameters for all the models is the placement on the ship deck. This could be defined to be anywhere relative to the centre of mass location.

The interface model has also been implemented in a Fortran function. This model is capable of limiting the magnitude of the interface force. As well, there is an option available to not even include the interface force at all. A visualization of the flow of the DEPSM simulation is provided in Figure 18. The simulation starts by calculating the ship motion at the position on the deck where the stability model is placed. Input data on the desired ship motion is provided from DEPSM.inp file. The executable then selects the subroutine for the stability model that is desired. It then calculates the desired load model. The stability and load models have input files that provide the physical parameters for the model. Finally the interface force model is calculated and the tension is then used for the stability and load models.

The DEPSM simulation runs very quickly such that a 5000 step simulation takes approximately 5 seconds to complete.

\section{$2.4 \quad$ VFD-RT Simulation Implementation}

The Virtual Flight Deck - Real Time (VFD-RT) is a simulation environment developed by Kin Wing Tsui in the Carleton University Applied Dynamics Laboratory several years ago. The motivation for the development of this simulation environment was to make programming of a distributed simulation easy for new users specializing in marine simulations. The full development of the software can be found in Reference [1]. This section will provide a brief overview of the software followed by the 


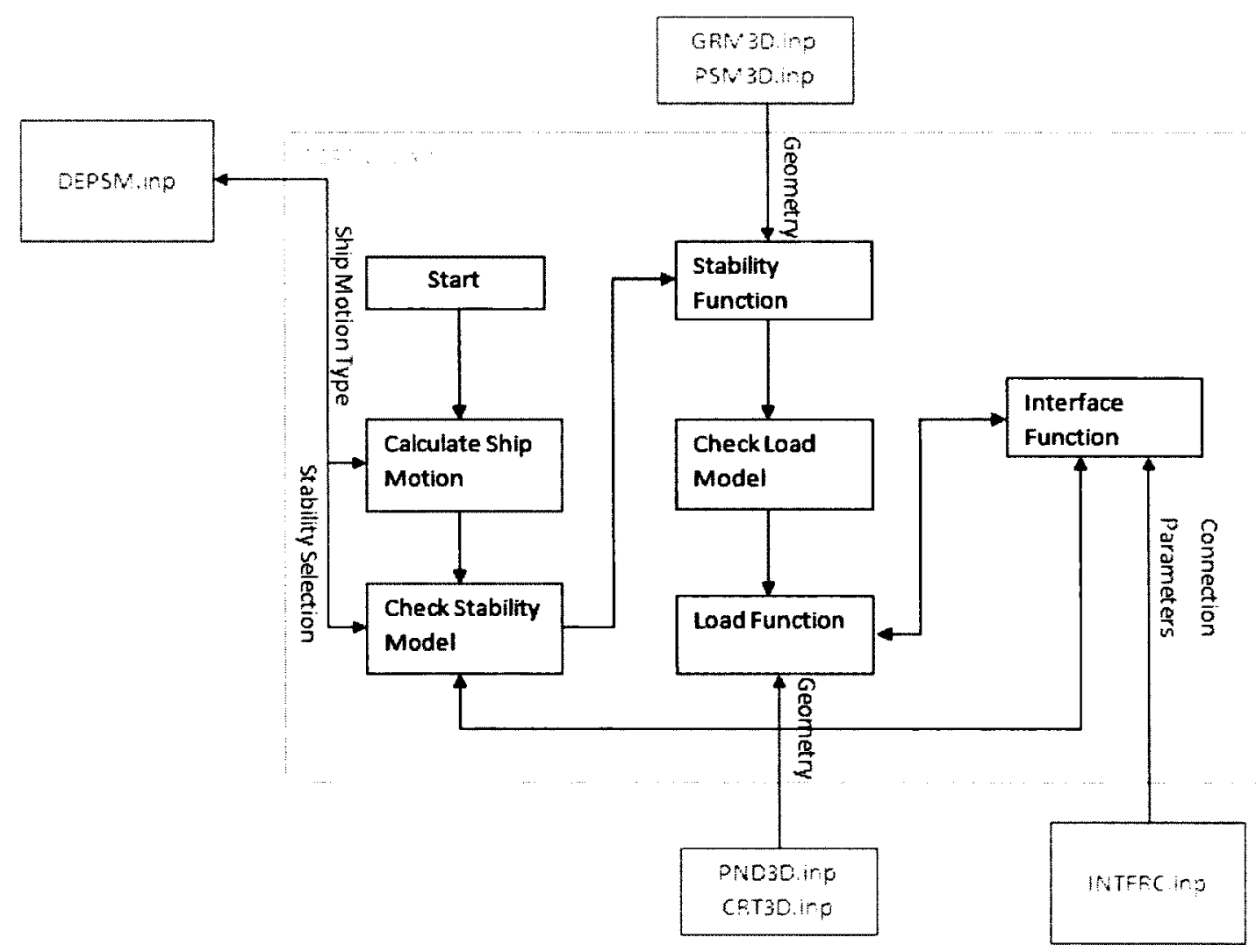

Figure 18: Visualization of DEPSM simulation environment and solution flow. 
implementation of the stability and load models in this simulation infrastructure.

The VFD-RT is a distributed simulation environment for simulation of maritime systems. This framework is similar to the common and very in-depth HLA simulation framework, STD 1516.2-2010. The use of the HLA architecture was a requirement asked by DRDC for the PSM project. Since the VFD-RT is a similar framework it was selected. The VFD-RT uses a provider and client method of determining communications. A provider is said to supply data for the other executables attached to the VFD-RT, where a client is said to take certain required data from the VFD-RT. In order to achieve the communications between executables, they all must be connected to the VFD-RT. All connections and communication within the VFD-RT are made using the Windows communication protocols. For example, in order to connect to the main VFD-RT module, it searches for all windows running that have the VFD-RT ID attached to them. Unlike the HLA, the executables attached to the VFD-RT do not need to be part of a federation, as well, the data sent between providers and clients does need to be contained within an eighteen-cell array and cannot be sent as a custom predefined class. A comparision between the VFD-RT and HLA can be seen in Figure 19. The data is transferred by copying the data to the windows clipboard, which is then picked up by the main VFD-RT program. The decision to define the data transfer size was made by the developer because he had assumed that the largest required data package that would need to be passed in the simulation would be the ship motions for displacement, velocity, and acceleration. This was chosen in order to maintain the simplicity for the user in creating new models. The challenge of knowing which data is coming from which executable connected to the VFD-RT is solved by assigning an ID number to any provider executable as it is connected to the VFD-RT. This way it can be known which executable the data is coming from, and therefore the data that the executable is providing. This also means that there is a requirement to know which executables will be needed for the simulation, and the 
order in which they will be connected to the VFD-RT.

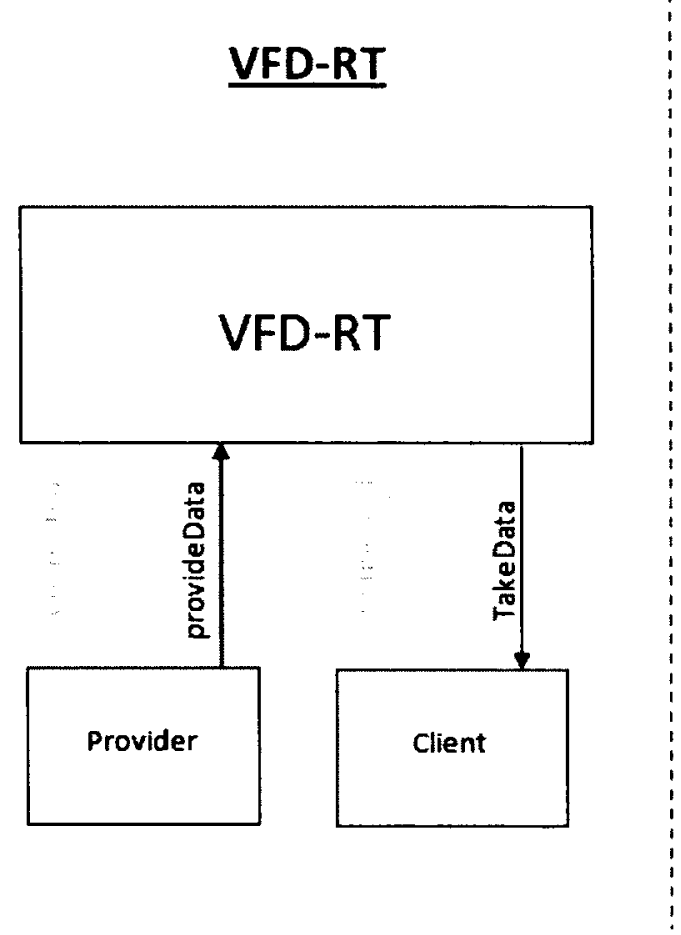

\section{HLA}

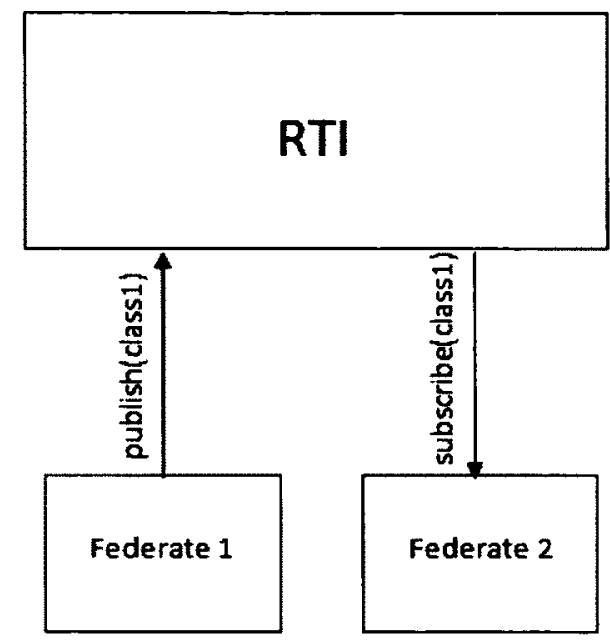

Figure 19: Connection and data transfer comparison between the VFD-RT and HLA architectures.

Since the VFD-RT is a similar distributed simulation environment to the HLA architecture, the postural stability and load models are implemented in the VFD-RT framework. It has also been proved that a VFD-RT provider or client can quickly be converted into an HLA federate if that is desired. Each of the stability models have been included as a VFD-RT provider/client. A provider/client is both a provider and client at the same time. It is able to connect to the VFD-RT to provide data as well as take the required data. A client is able to take data from two different provider sources. The simulation diagram is provided in Figure 20. The stability models require the ship motion input, which is provided from a ship motion provider, as well as the tension vector of the interface link. These models provide the position and velocity of the attachment point of the link to calculate the interface force. The 
load models also take the ship motion data and the tension vector on the link. They provide the location and velocity of the attachment point to calculate the tension vector. The interface force requires the data on the attachment points of each of the models, and provides the tension vector.

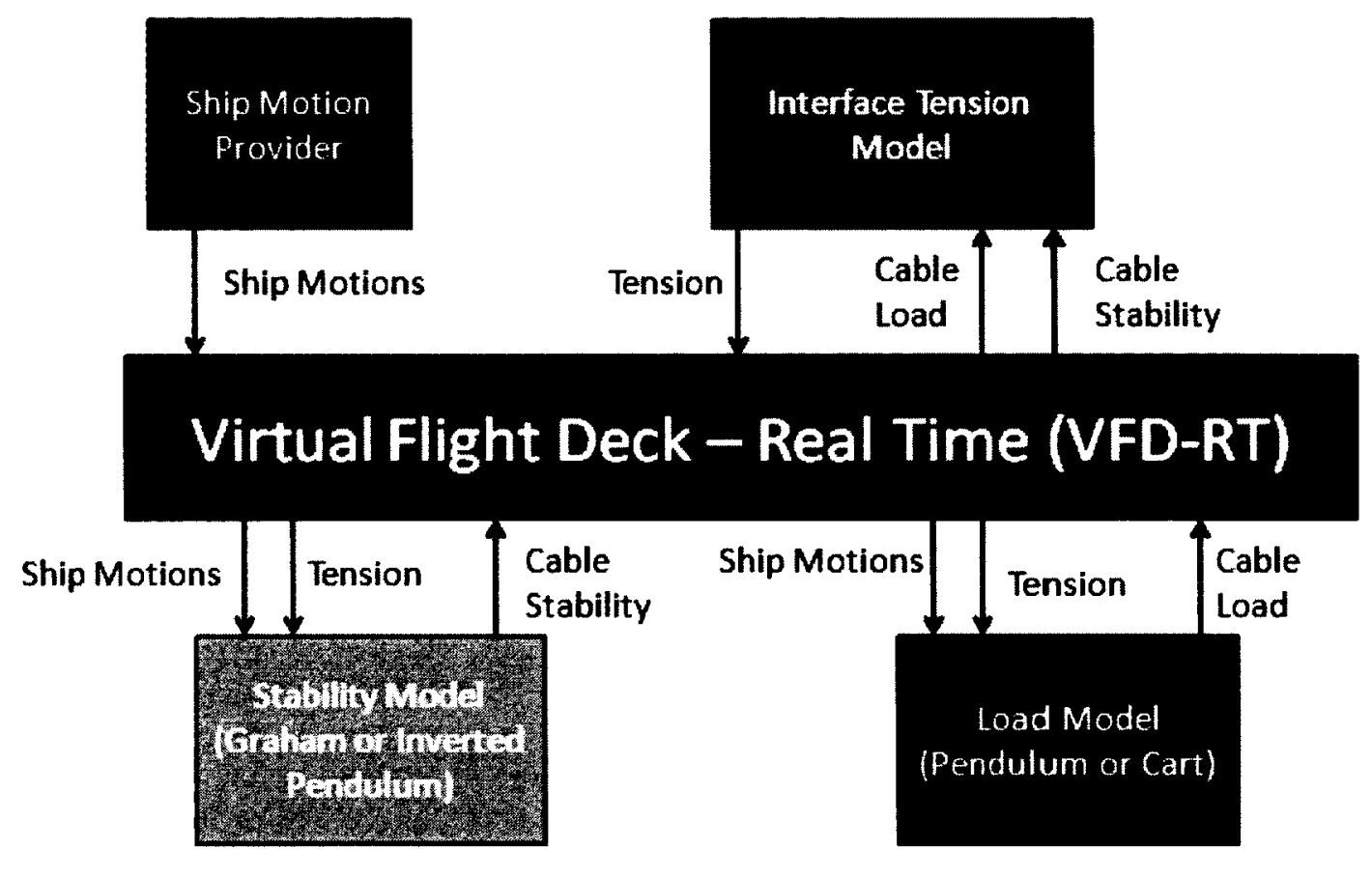

Figure 20: Connection and data transfer between VFD-RT executables.

Each of the VFD-RT federates has a Fortran subroutine linked to the executable. This is accomplished using Microsoft Visual Studio 2003 which applies a dynamic link to the object file. This ensures that the model being used is exactly consistent between the DEPSM and VFD-RT simulations. They are both using the same subroutines to calculate the motions and forces. This also provides a method of verifying the VFD-RT simulation with the DEPSM simulation. 


\subsection{Computer Simulation Verification}

Using the two development environments, DEPSM and VFD-RT, allows verification of the VFD-RT simulation using the DEPSM simulation. The verification was performed using general ship motion representative of a frigate operating in an elevated sea state.

In order for the ship motion to be provided to the VFD-RT, a general motion provider must be used. This is one of many basic programs for performing general tasks such as sending general ship motion from a text file, as well as viewing the data in real time. These programs have all been previously validated and are known to function correctly.

There were several cases tested in order to verify the VFD-RT simulation environment. To satisfy the verification, it was required that all results be exactly the same. This is because, as mentioned previously, the VFD-RT programs are using the same subroutines as the DEPSM simulation to calculate the model dynamics. This verifcation was to determine if the communication between the executables was working correctly to and from the VFD-RT and that the subroutines were being executed in the correct order. The results from testing the Graham model using the DEPSM environment and the VFD-RT environment can be seen in Figures 21 through 26. It can be seen that the results from the simulations run using the DEPSM environment and the VFD-RT environment are overlapping and therefore the same. Similar results were obtained for other permutations of the available models. Therefore, the simulations have been verified against each other. 


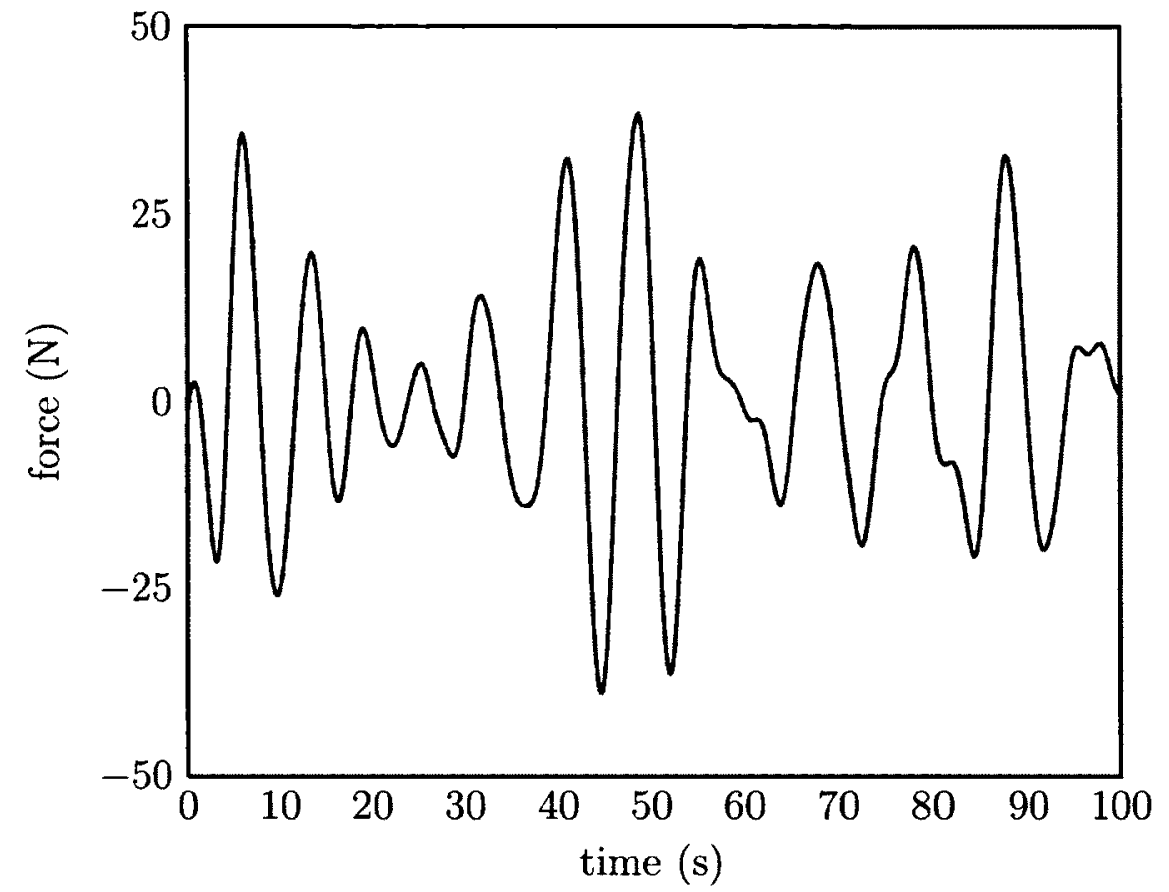

Figure 21: Longitudinal force $\left(F_{x}\right)$ computed using GRM3D implemented in the VFD-RT (solid) and the DEPSM simulation environments (dashed).

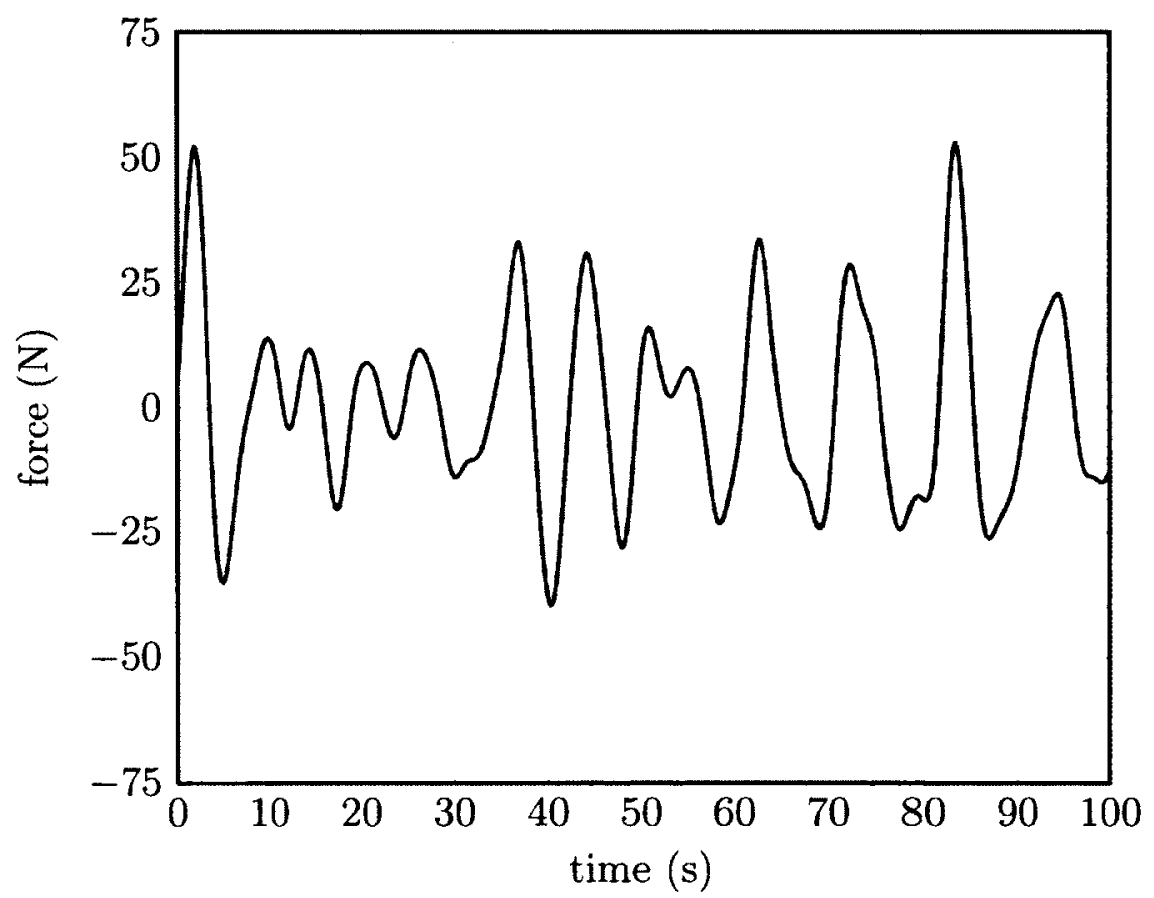

Figure 22: Lateral force $\left(F_{y}\right)$ computed using GRM3D implemented in the VFD-RT (solid) and the DEPSM simulation environments (dashed). 


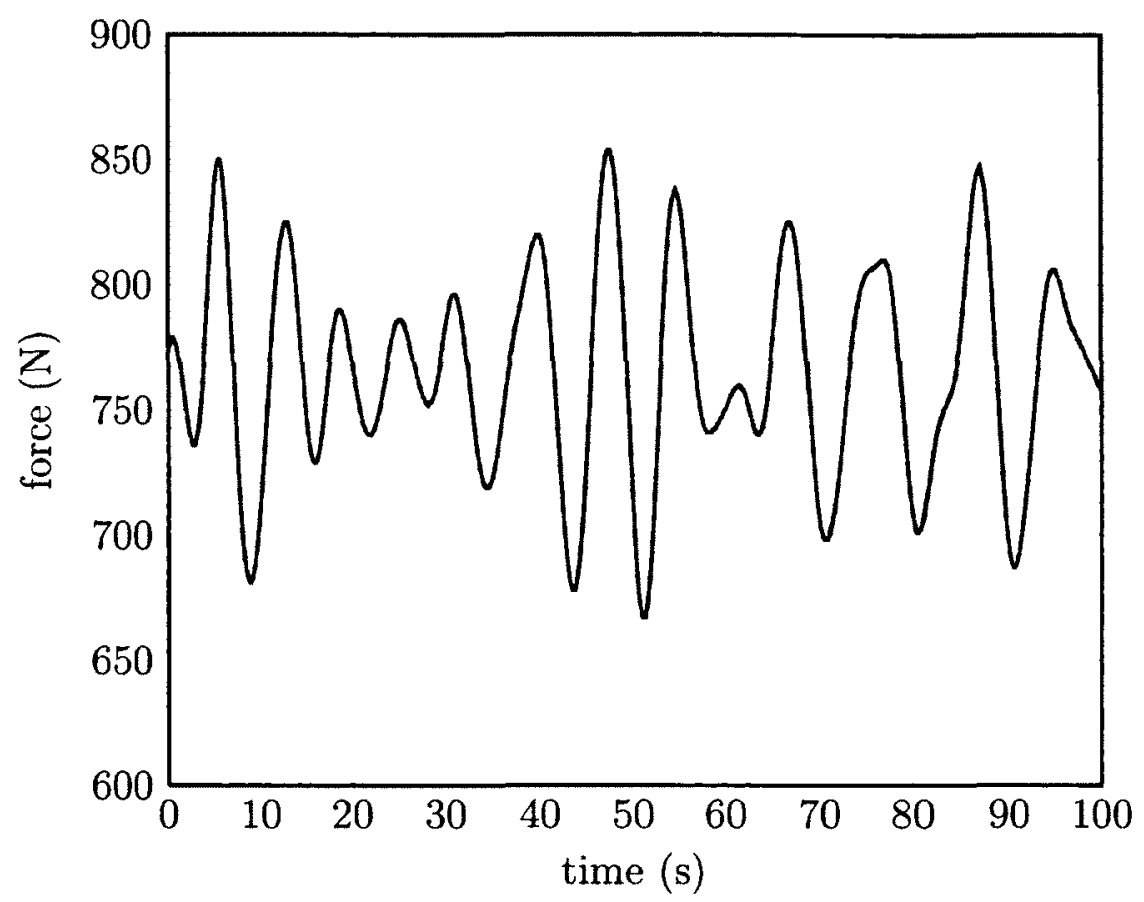

Figure 23: Vertical force $\left(F_{z}\right)$ computed using GRM3D implemented in the VFD-RT (solid) and the DEPSM simulation environments (dashed).

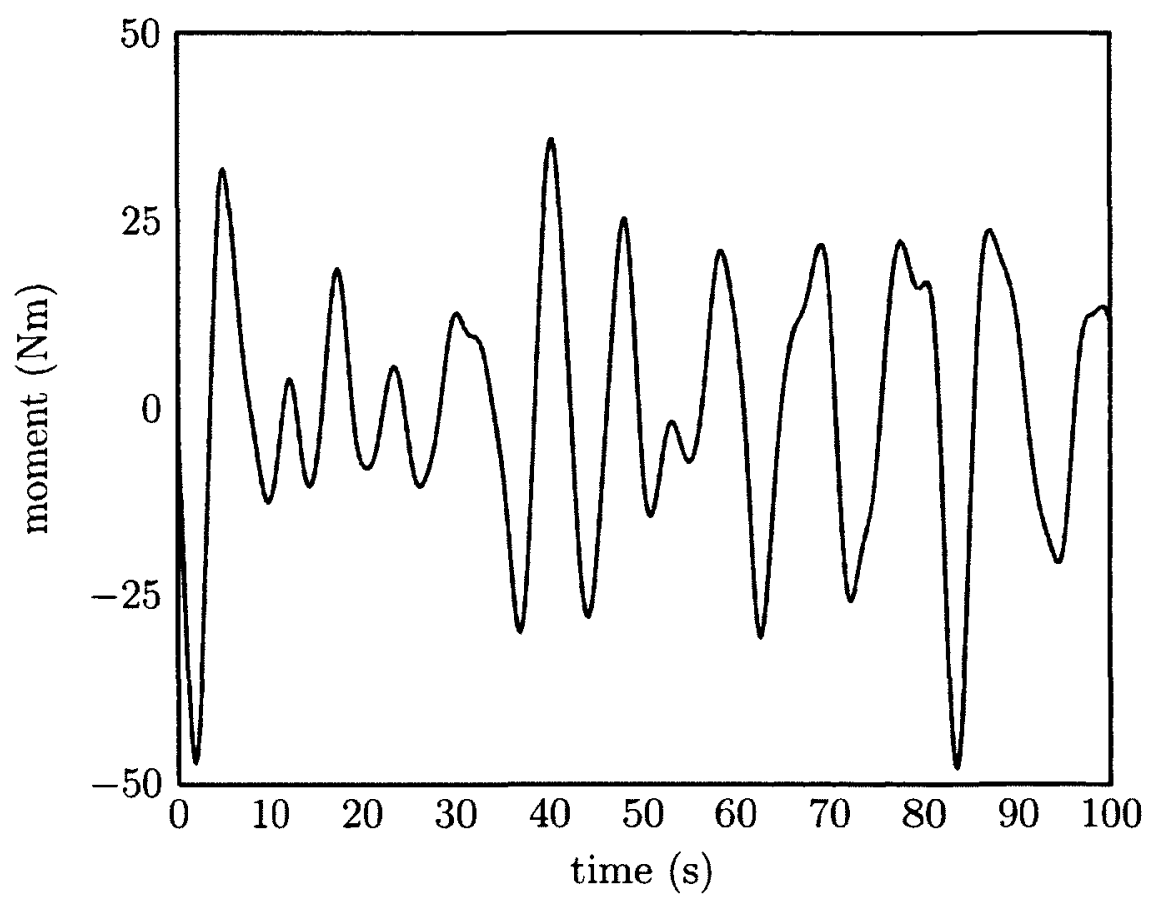

Figure 24: Longitudinal moment $\left(M_{x}\right)$ computed using GRM3D implemented in the VFD-RT (solid) and the DEPSM simulation environments (dashed). 


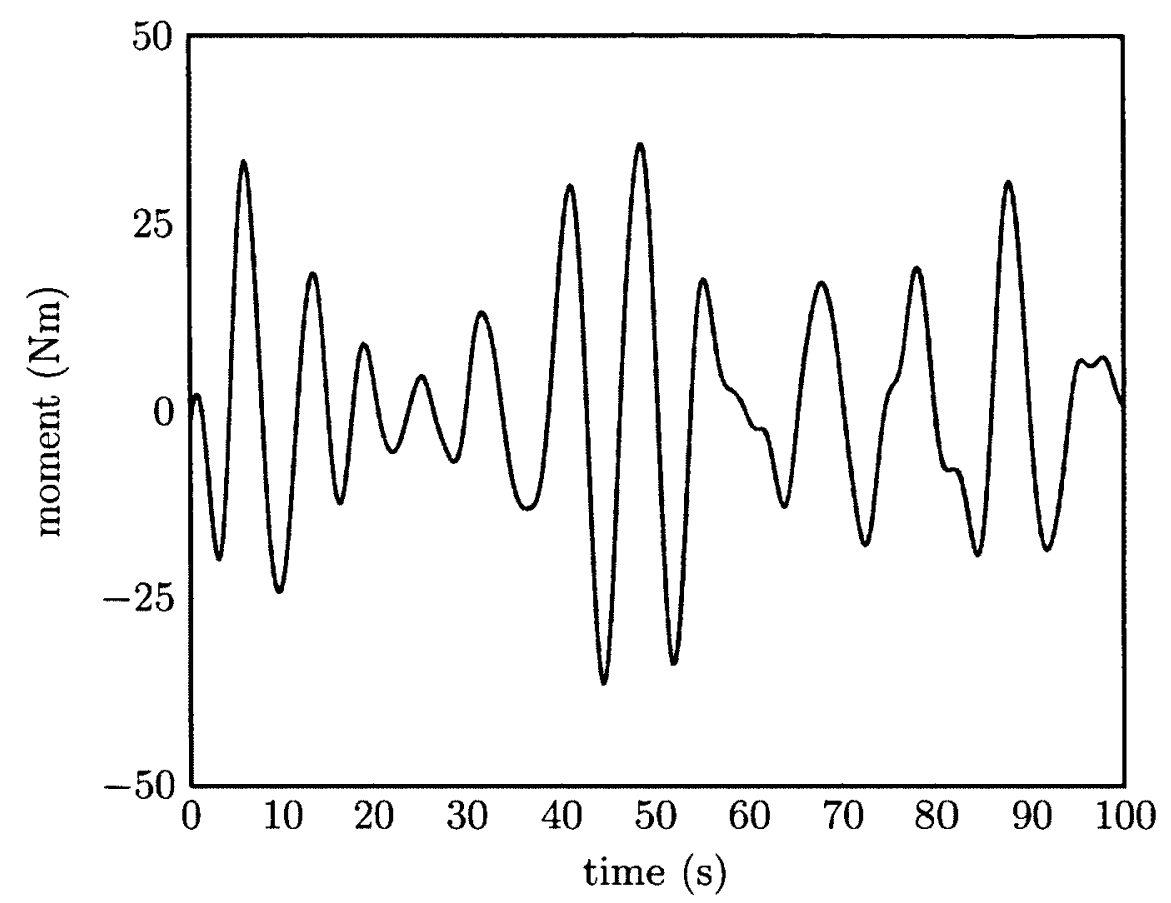

Figure 25: Lateral moment $\left(M_{y}\right)$ computed using GRM3D implemented in the VFD-RT (solid) and the DEPSM simulation environments (dashed).

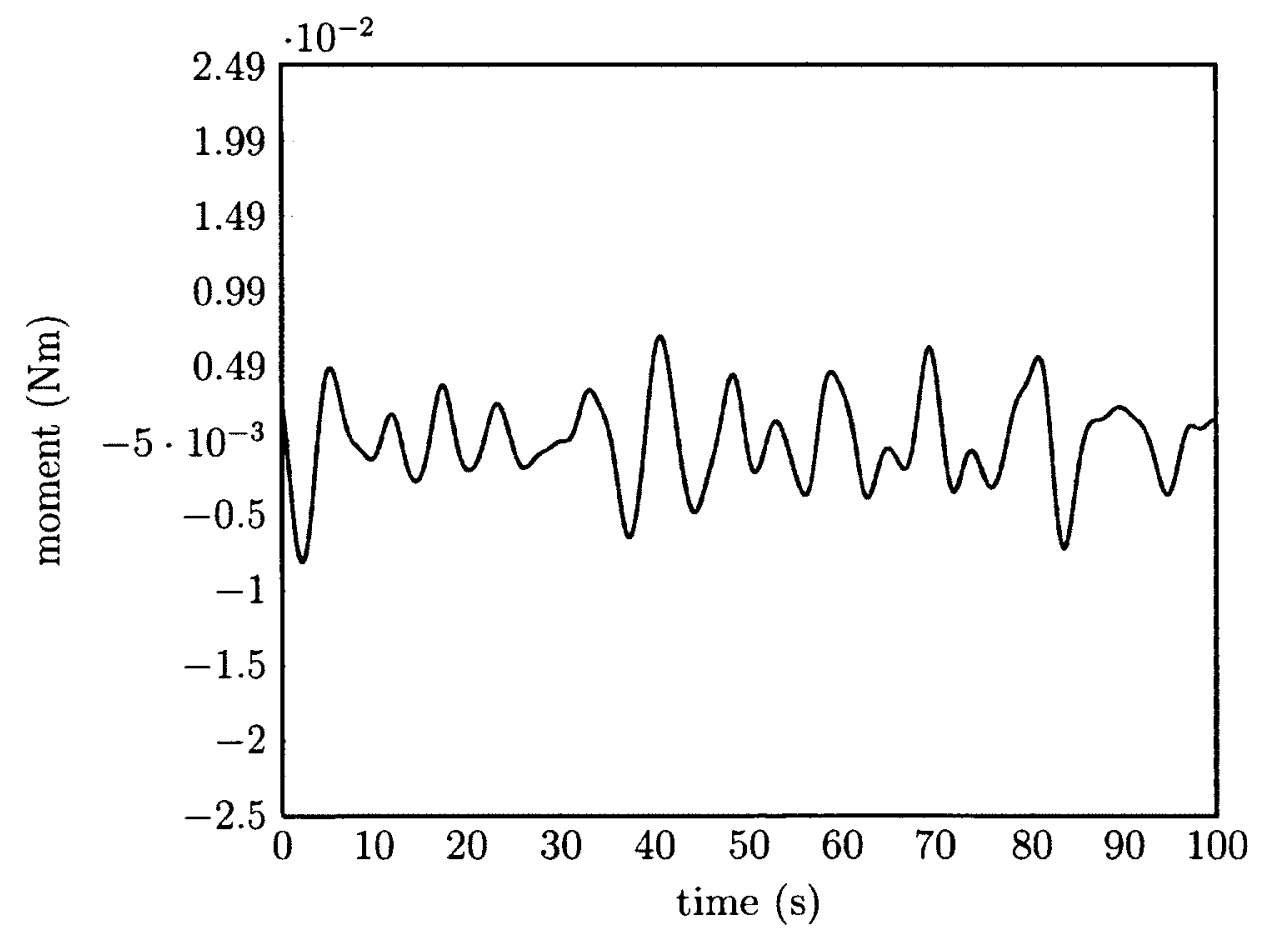

Figure 26: Vertical moment $\left(M_{z}\right)$ computed using GRM3D implemented in the VFD-RT (solid) and the DEPSM simulation environments (dashed). 


\section{Chapter 3}

\section{Model Validation}

\subsection{Overview}

This chapter discusses the validation experiments performed to validate the theoretical models. There are two methods that were used to validate the Graham stability models. The first method involved comparing the results from the Graham model to that of the locked inverted pendulum model developed by Langlois [44]. It was assumed that by locking the articulation at the pendulating joint of the inverted pendulum model, it would collapse into the Graham model, and therefore produce similar results. The second method of validation was through experimentation using full-scale physical hardware designed and built for the purpose of validating these models. The physical models were constructed in order to be attached to a six-degreeof-freedom motion platform. The force and moment data at the base of the model are collected in the experiments and can be compared to the results from the simulation for validation. Since the cart model is not stable without any control, the model was stabilized using a linear spring and damper in the translational direction, and a rotational spring and damper about the rotation axis. The validation is obtained by exciting the model with a sinusoidal linear or angular displacement at several 
frequencies and comparing the results to known frequency response curves. Validation of the coupled models is obtained by comparing the results from simulation and the measured experimental results. The next section provides the validation of the Graham stability model compared to the inverted pendulum model, followed by the vibration analysis for the cart validation, and finally the validation of the Graham model through experimentation.

\subsection{Validation of the Graham Stability Model with the Locked Inverted Pendulum}

The formulation of the GRM3D postural stability model can be thought of as a special case of a more general articulated inverted pendulum postural stability model with all articulation degrees of freedom constrained. Therefore, comparing GRM3D with a model such as PSM3D with its two articulation joints locked provides a computational means of partially validating GRM3D. Details of the PSM3D inverted pendulum model as well as its complete derivation are documented in Reference [44]. Briefly, the spatial inverted pendulum model is a postural stability model. The model is derived in three dimensions assuming a single link inverted pendulum with the two rotation centres located at the attachment point at the base. The model is free to rotate about the two rotation axes. In order for the model to be stable, a simple active controller has been added. The model solves for the interface forces at the attachment point, the angle of the model with respect to the two rotation axes, and the interface moments. To avoid reformulating the PSM3D model, the final set of governing dynamic equations, that conventionally are solved to obtain the three com-

ponents of the interface force $\left(F_{x}, F_{y}\right.$, and $\left.F_{z}\right)$, the interface moment $\left(M_{z}\right)$, and the 
Table 3: Simulation parameters used for validating GRM3D against PSM3D

\begin{tabular}{cc}
\hline Parameter & Value \\
\hline$m$ & $78 \mathrm{~kg}$ \\
$I_{x x}$ & $47.2 \mathrm{~kg} \mathrm{~m}^{2}$ \\
$I_{y y}$ & $47.2 \mathrm{~kg} \mathrm{~m}^{2}$ \\
$I_{z z}$ & $1 \mathrm{~kg} \mathrm{~m}{ }^{2}$ \\
$\mathbf{r}_{C G / A}^{M O}$ & $\{0.00 .00 .9\}^{T} \mathrm{~m}$ \\
$\mathrm{~g}$ & $9.81 \mathrm{~m} / \mathrm{sec}^{2}$ \\
\hline
\end{tabular}

relative angular accelerations about each of the two orthogonal axes forming the articulation joint $\left(\ddot{\theta}_{x}\right.$ and $\left.\ddot{\theta}_{y}\right)$, were reformulated for the constrained case by moving the now known $\ddot{\theta}_{x}$ and $\ddot{\theta}_{y}$ to the right hand side of the equation and relocating the previously known ankle moments $\left(M_{x}\right.$ and $\left.M_{y}\right)$ to the left hand side. With the articulation locked, $\theta_{x}$ and $\theta_{y}$ as well as their time derivatives are always zero. With the reformulation of the final equations, the PSM3D model could be used to solve for the six force and moment components evaluated by the GRM3D model. Further, in making this change, the PSM3D model no longer required numerical integration but rather, in principle, became a simple function evaluation. Numerical integration of the model was retained to minimize the extent of recoding of the PSM3D model required to include the option for locking the articulation joint.

For the computational validation, simulation conditions were set up using the physical parameters listed in Table 3 corresponding to those originally proposed by Graham [35] and typical ship motion representative of a frigate operating on an oblique heading, at moderate speed, in sea state four conditions. Using the same initial conditions, both the GRM3D and PSM3D models were run for a 60 second duration.

The simulation results for each force component direction obtained from both 
models are presented in Figures 27 through 32. As it can be seen from the simulation results, relatively close agreement is found between the full dynamic solution provided by PSM3D and the quasi-static solution obtained from GRM3D. Of interest is the fact that identical results are obtained for the yaw moment $\left(M_{z}\right)$. Differences between models are currently attributed to the method used for constraining the articulation joint in the PSM3D model. This will be discussed further in Chapter 5.

\subsection{Computational Validation of the Cart Load Model}

The cart model is computationally validated by separating the translational and rotational degrees of freedom and validating them separately. The cart model is stabilized by adding a translational directed spring and damper element along the translational axis between the deck and cart; as well as a similar torsional directed element between the lower and upper cart bodies. The resulting stable model is validated by comparing the forced frequency responses of the system to the theoretical responses.

Adding the spring and damper for the translating body added external forces being applied to the body. This required the modification of Equation 32 with the addition of the spring and damper forces resulting in

$$
\sum \vec{F}_{B}^{I N}=\vec{F}_{B R}^{I N}-\vec{F}_{T R}^{I N}-m_{1} \vec{g}^{I N}+c \vec{v}_{B / A}^{M O}+k \vec{r}_{B / A}^{M O}
$$

This modification is carried through the equations presented previously in Section 2.2.3 and the resulting translational governing equation was found.

The physical parameters for validating this model are given in Table 4 . These values do not have to be compared to any physical apparatus, thus the values were 


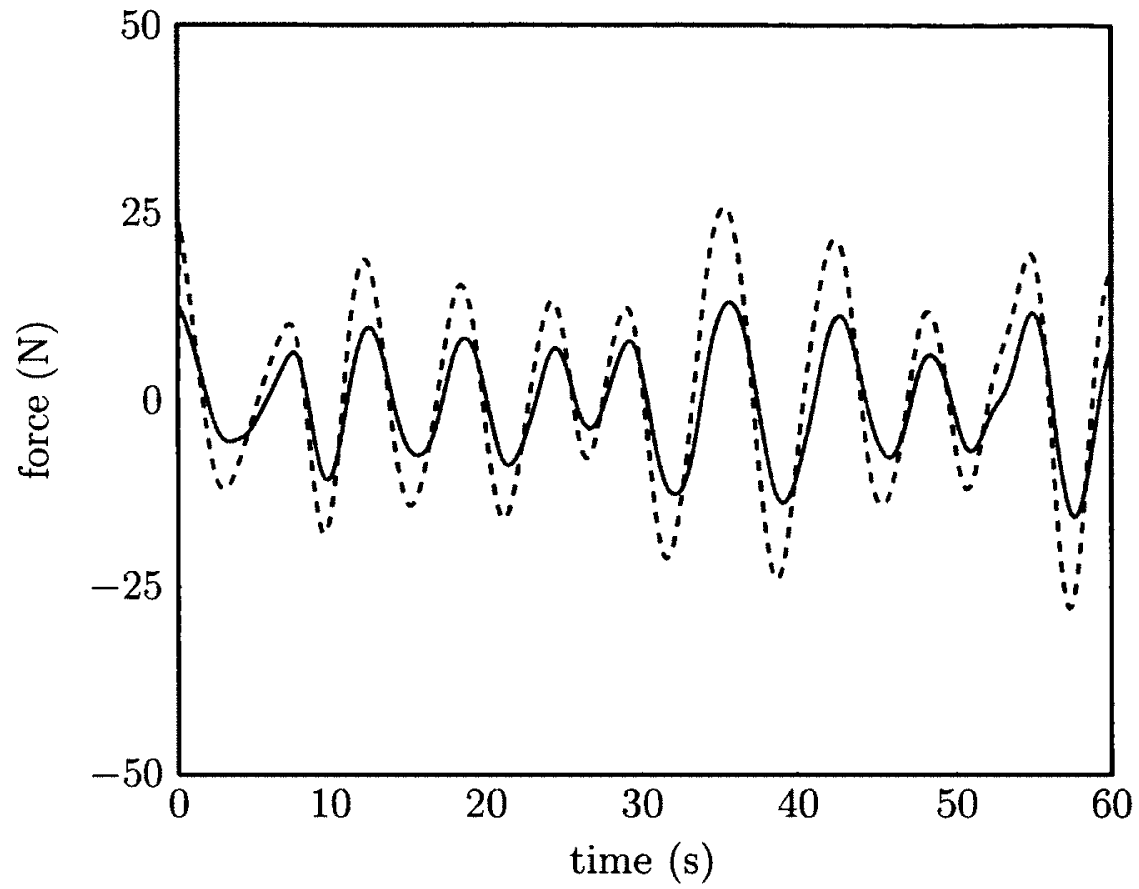

Figure 27: Longitudinal force $\left(F_{x}\right)$ for typical ship motion computed using GRM3D (dashed) and PSM3D (solid).

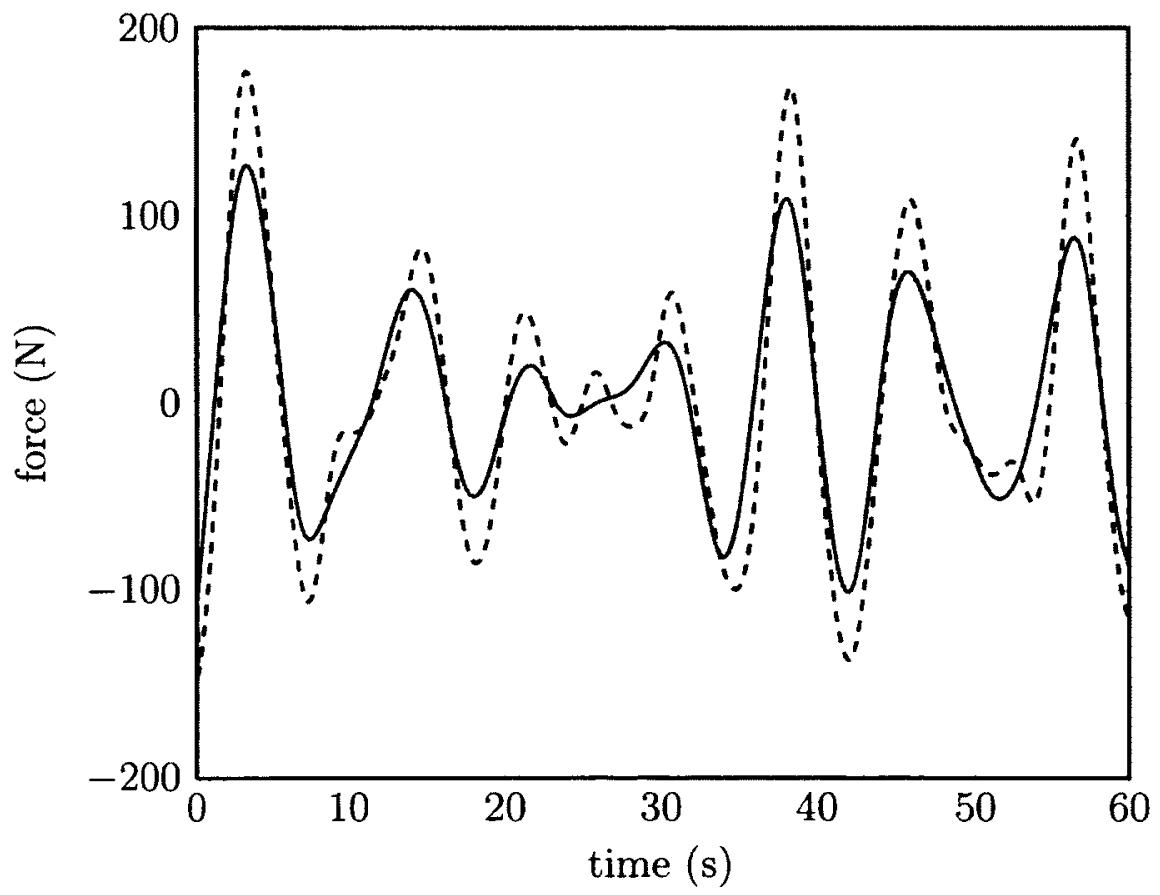

Figure 28: Lateral force $\left(F_{y}\right)$ for typical ship motion computed using GRM3D (dashed) and PSM3D (solid). 


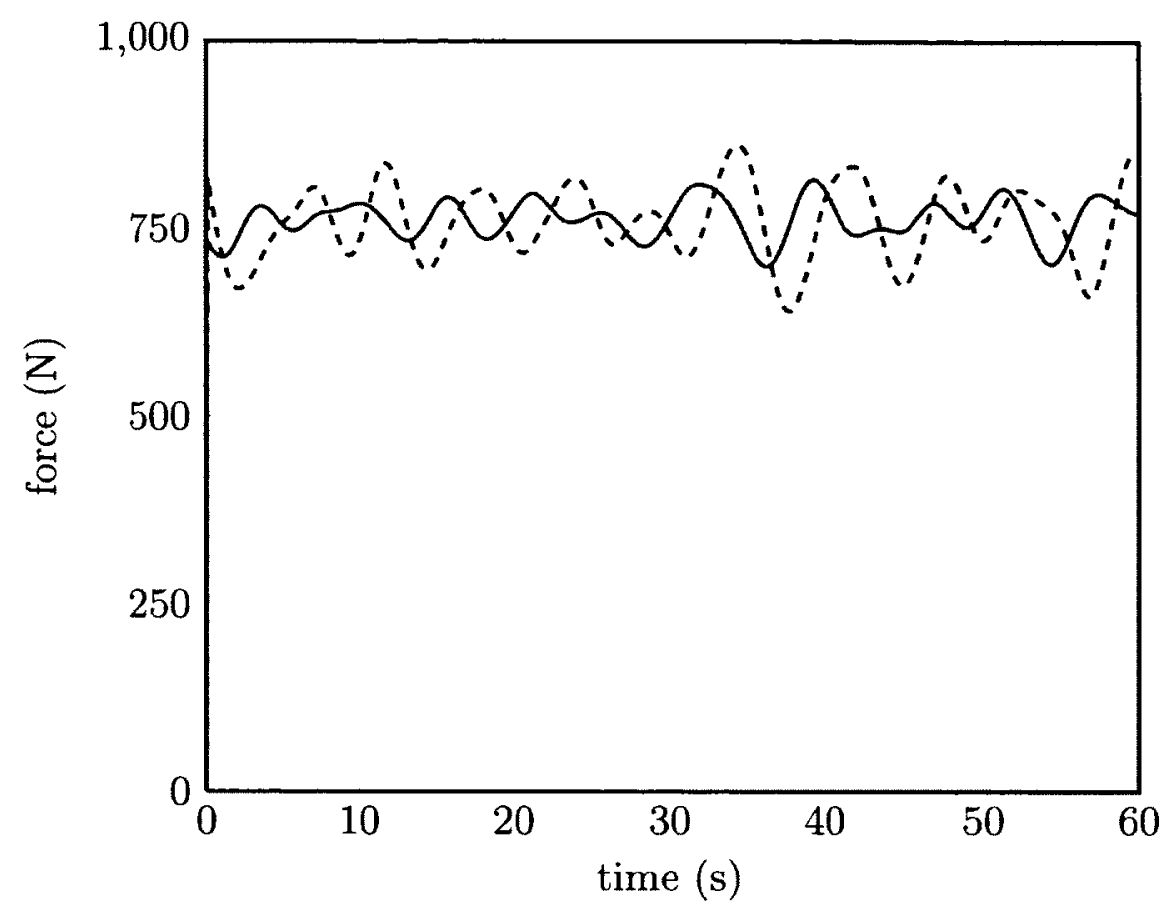

Figure 29: Vertical force $\left(F_{z}\right)$ for typical ship motion computed using GRM3D (dashed) and PSM3D (solid).

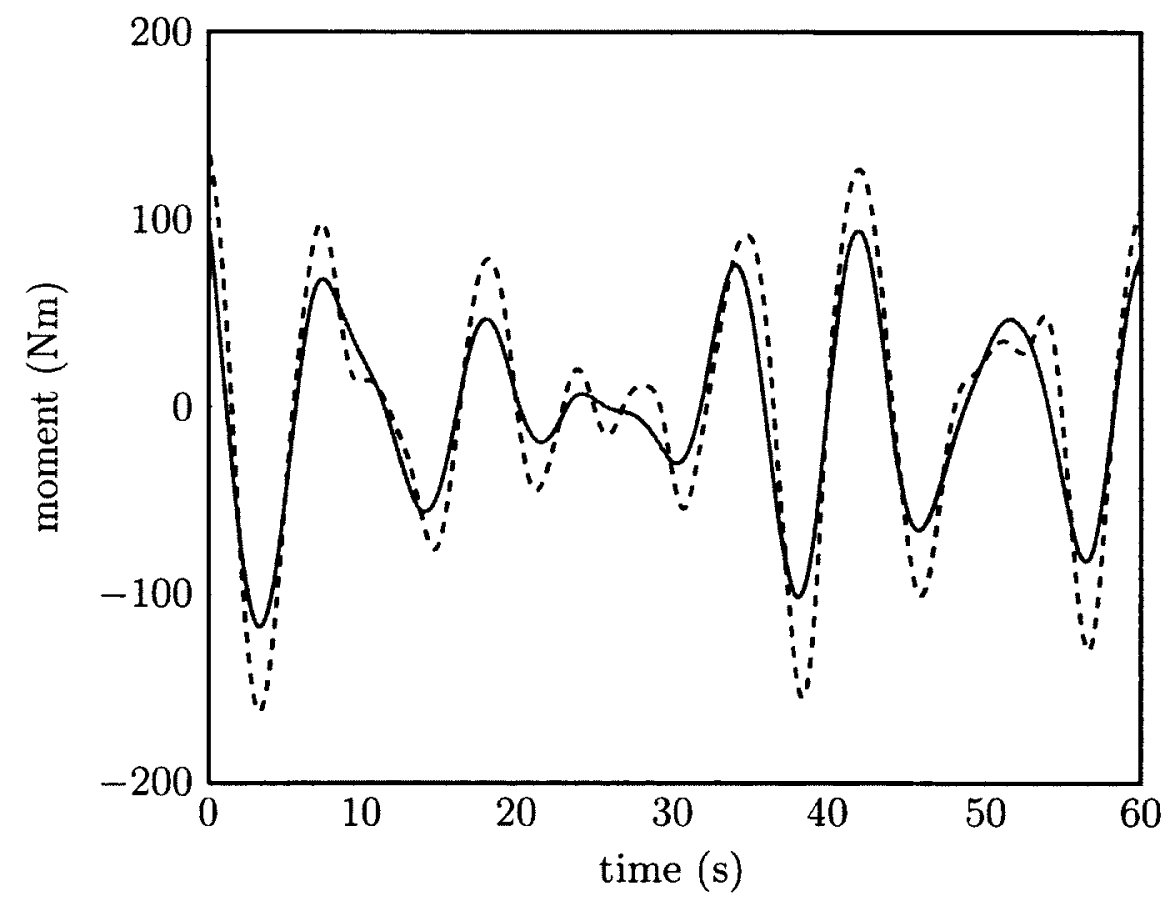

Figure 30: Longitudinal moment $\left(M_{x}\right)$ for typical ship motion computed using GRM3D (dashed) and PSM3D (solid). 


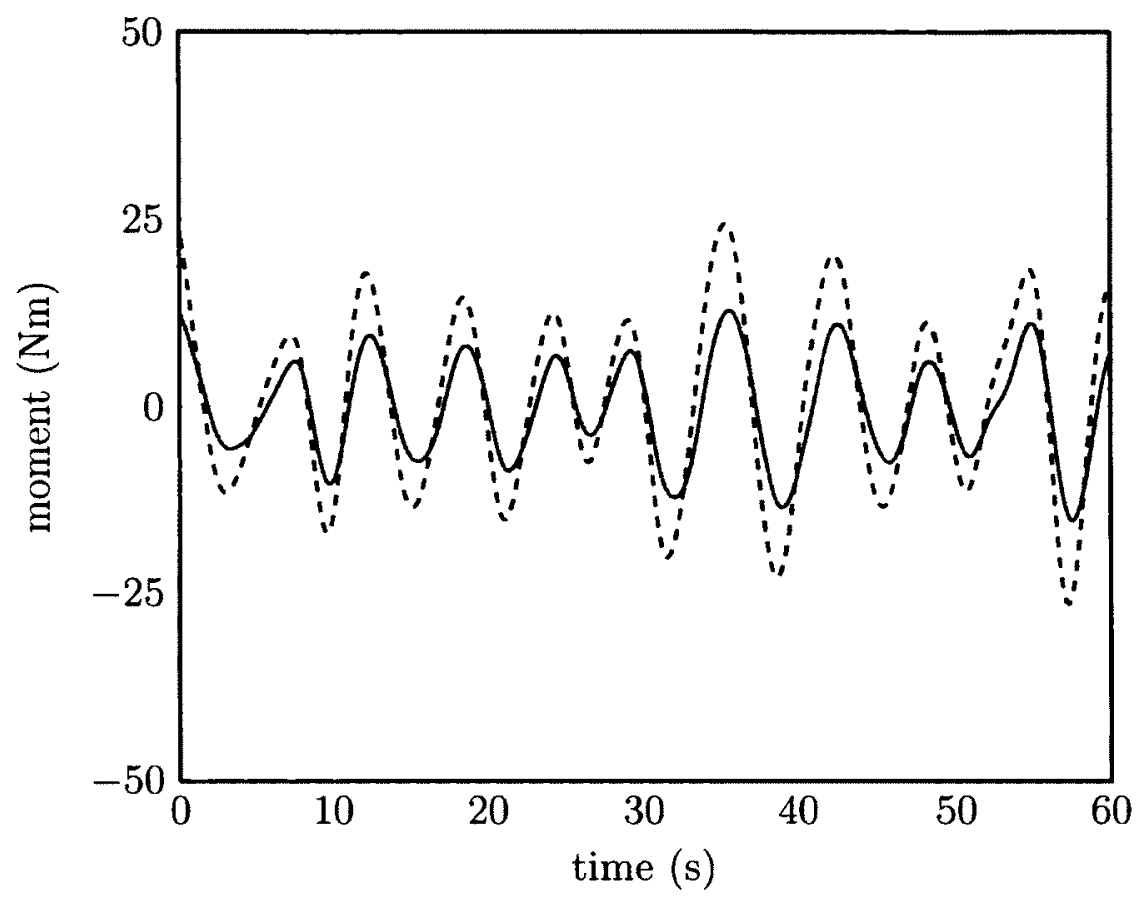

Figure 31: Lateral moment $\left(M_{y}\right)$ for typical ship motion computed using GRM3D (dashed) and PSM3D (solid).

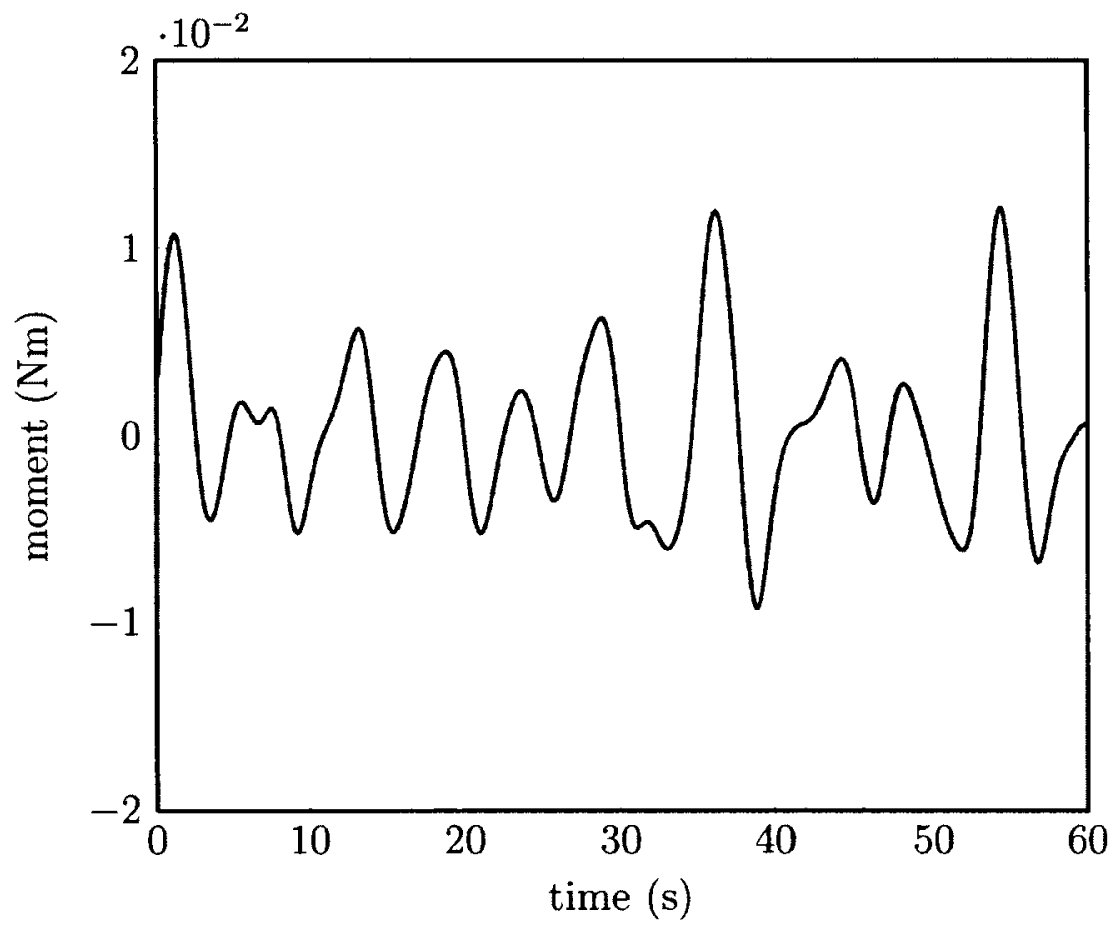

Figure 32: Vertical moment $\left(M_{z}\right)$ for typical ship motion computed using GRM3D (dashed) and PSM3D (solid). 
Table 4: Simulation parameters used for CRT3D validation

\begin{tabular}{cc}
\hline Parameter & Value \\
\hline$m_{1}$ & $78 \mathrm{~kg}$ \\
$m_{2}$ & $22 \mathrm{~kg}$ \\
$I_{x x 1}$ & $10 \mathrm{~kg} \mathrm{~m}^{2}$ \\
$I_{y y 1}$ & $10 \mathrm{~kg} \mathrm{~m}^{2}$ \\
$I_{z z 1}$ & $1 \mathrm{~kg} \mathrm{~m}^{2}$ \\
$I_{x x 2}$ & $10 \mathrm{~kg} \mathrm{~m}^{2}$ \\
$I_{y y 2}$ & $10 \mathrm{~kg} \mathrm{~m}^{2}$ \\
$I_{z z 2}$ & $1 \mathrm{~kg} \mathrm{~m}^{2}$ \\
$\mathrm{r}_{C G / A}^{M O}$ & $\{0.00 .00 .9\}^{T} \mathrm{~m}$ \\
$\mathrm{~g}$ & $9.81 \mathrm{~m} / \mathrm{sec}^{2}$ \\
\hline
\end{tabular}

chosen for ease of use. The simulation was run for three different damping ratios. For each damping ratio the simulation was run for five frequencies. The frequencies were selected to be close to the natural frequency in order to see the increase in amplitude as well as the expected change of phase above the system natural frequency.

A similar method was used in order to validate the rotational degree of freedom of the model. This method stabilized the rotational degree of freedom by adding a torsional spring and damper. This resulted in an added external moment to Equation 57. The simulation was run for three different damping ratios. For each damping ratio the simulation was run for five frequencies. The frequencies were selected to be close to the natural frequency in order to see the increase in amplitude as well as the expected change of phase above the system natural frequency.

The calculated frequencies and amplitudes were compared to the expected results from the solution of an analogous single degree-of-freedom spring damper system. The translational results have been tabulated in Tables 5 through 7 . The rotational 
Table 5: Results from translational forced frequency excitation on the cart for the case where $\zeta=0.35$

\begin{tabular}{ccccc}
\hline$m=20 \mathrm{~kg}$ & $k=1000 \mathrm{~N} / \mathrm{m}$ & $c=100 \mathrm{Ns} / \mathrm{m}$ & $f_{n}=1.1 \mathrm{rad} / \mathrm{s}$ & $\zeta=0.35$ \\
\hline $\begin{array}{c}\text { Forced Freq. } \\
\mathrm{rad} / \mathrm{s}\end{array}$ & Freq. Ratio & Freq. Ratio & Amp. Ratio & Amp. Ratio \\
\hline 0.8 & 0.77 & 0.73 & 1.27 & 1.3 \\
1.0 & 0.86 & 0.91 & 1.22 & 1.2 \\
1.4 & 1.24 & 1.27 & 0.95 & 1.0 \\
1.8 & 1.58 & 1.64 & 0.55 & 0.60 \\
2.2 & 1.97 & 2.00 & 0.34 & 0.35 \\
\hline
\end{tabular}

results have been tabulated in Tables 8 through 10. Comparing the simulation results to the expected theoretical values in the tables, the results are found to be similar and thus have validated the cart model.

As well as the constant frequency tests, a frequency sweep was performed to see the frequency response of the system over frequencies ranging from $0.1 \mathrm{rad} / \mathrm{s}$ to $3.0 \mathrm{rad} / \mathrm{s}$. The frequency sweep was performed with a spring constant of $1000 \mathrm{~N} / \mathrm{m}$, a damping coefficient of $50 \mathrm{Ns} / \mathrm{m}$, and a total cart mass of $20 \mathrm{~kg}$ in the translational direction. The resulting frequency response can be seen in Figure 33. It should be noted that at the natural frequency the amplitude multiplication factor should be approximately 2.8 , which can be seen at the peak of the graph. A similar frequency sweep was performed for the rotational degree of freedom. For this test the forcing frequencies ranged from $0.1 \mathrm{rad} / \mathrm{s}$ to $3.0 \mathrm{rad} / \mathrm{s}$ with a spring constant of $1000 \mathrm{Nm} / \mathrm{rad}$, a damping coefficient of $25 \mathrm{Nsm} / \mathrm{rad}$, and a total cart moment of inertia of $20 \mathrm{kgm}^{2}$ in the rotational direction. The rotational frequency response can be seen in Figure 34 . Again, the results are consistent with those expected. 


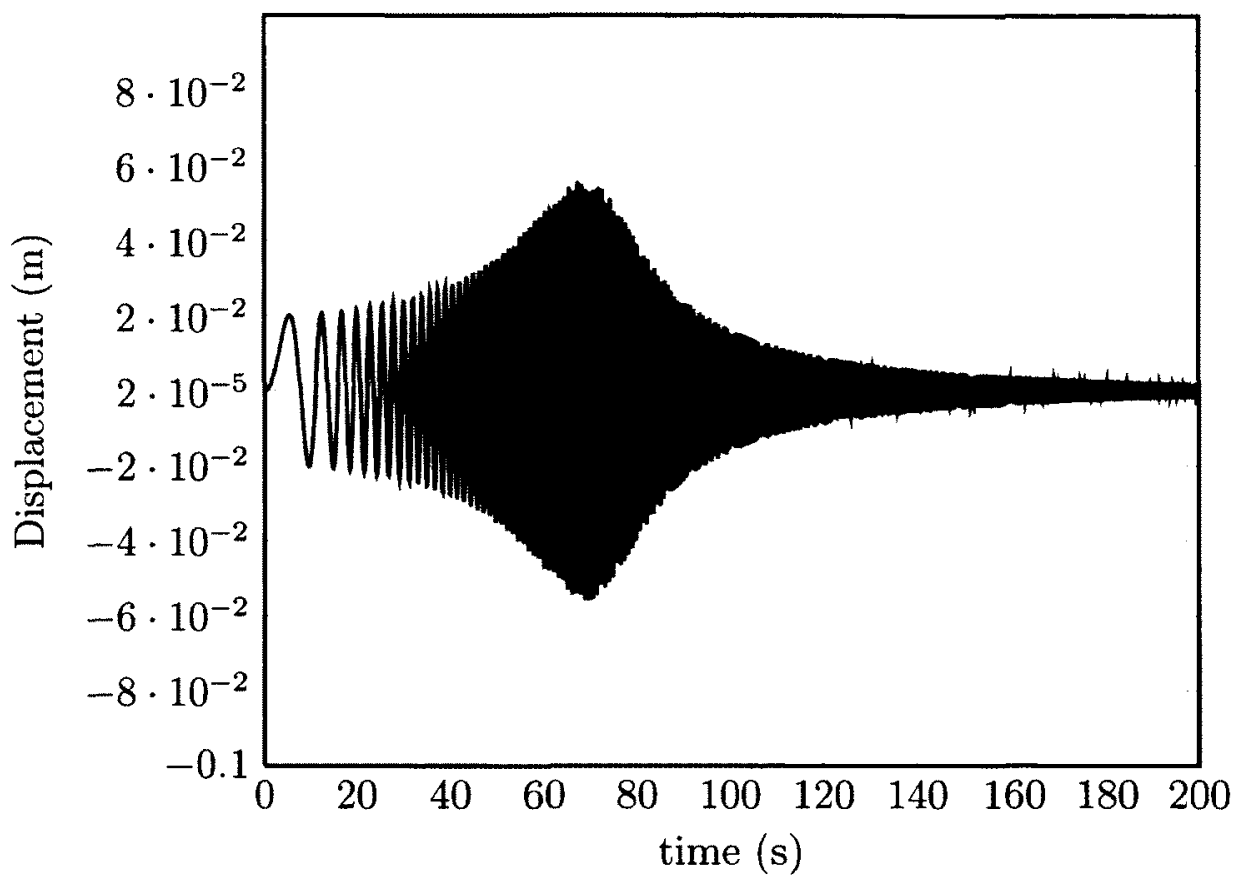

Figure 33: Frequency response for the cart model stabilized with a spring and damper in translation for the case where $\zeta=0.17$.

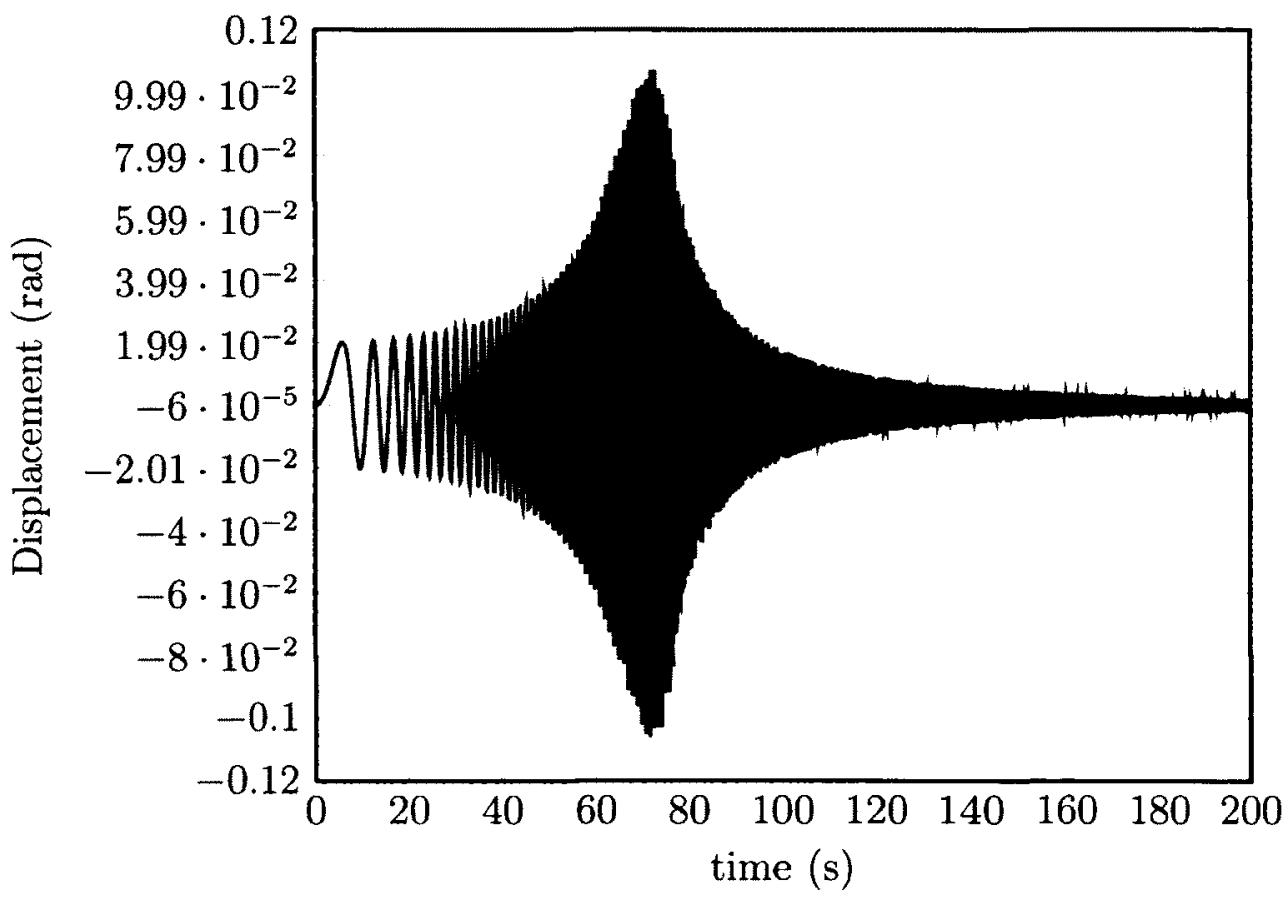

Figure 34: Frequency response for the cart model stabilized with a spring and damper in rotation for the case where $\zeta=0.085$. 
Table 6: Results from translational forced frequency excitation on the cart for the case where $\zeta=0.5$

\begin{tabular}{ccccc}
\hline$m=20 \mathrm{~kg}$ & $k=2000 \mathrm{~N} / \mathrm{m}$ & $c=200 \mathrm{Ns} / \mathrm{m}$ & $f_{n}=1.6 \mathrm{rad} / \mathrm{s}$ & $\zeta=0.5$ \\
\hline $\begin{array}{c}\text { Forced Freq. } \\
\mathrm{rad} / \mathrm{s}\end{array}$ & $\begin{array}{c}\text { Freq. Ratio } \\
\text { Simulated }\end{array}$ & $\begin{array}{c}\text { Freq. Ratio } \\
\text { Calculated }\end{array}$ & $\begin{array}{c}\text { Amp. Ratio } \\
\text { Simulated }\end{array}$ & $\begin{array}{c}\text { Amp. Ratio } \\
\text { Calculated }\end{array}$ \\
\hline 0.8 & 0.47 & 0.50 & 1.14 & 1.1 \\
1.0 & 0.65 & 0.63 & 1.24 & 1.2 \\
1.4 & 0.90 & 0.88 & 1.22 & 1.25 \\
1.8 & 1.05 & 1.13 & 0.92 & 0.90 \\
2.2 & 1.29 & 1.38 & 0.61 & 0.65 \\
\hline
\end{tabular}

Table 7: Results from translational forced frequency excitation on the cart for the case where $\zeta=0.25$

\begin{tabular}{ccccc}
\hline$m=20 \mathrm{~kg}$ & $k=2000 \mathrm{~N} / \mathrm{m}$ & $c=100 \mathrm{Ns} / \mathrm{m}$ & $f_{n}=1.6 \mathrm{rad} / \mathrm{s}$ & $\zeta=0.25$ \\
\hline $\begin{array}{c}\text { Forced Freq. } \\
\mathrm{rad} / \mathrm{s}\end{array}$ & $\begin{array}{c}\text { Freq. Ratio } \\
\text { Simulated }\end{array}$ & $\begin{array}{c}\text { Freq. Ratio } \\
\text { Calculated }\end{array}$ & $\begin{array}{c}\text { Amp. Ratio } \\
\text { Simulated }\end{array}$ & $\begin{array}{c}\text { Amp. Ratio } \\
\text { Calculated }\end{array}$ \\
\hline 0.8 & 0.51 & 0.50 & 1.26 & 1.25 \\
1.0 & 0.62 & 0.63 & 1.62 & 1.55 \\
1.4 & 0.85 & 0.88 & 1.97 & 2.00 \\
1.8 & 1.14 & 1.13 & 1.87 & 1.90 \\
2.2 & 1.32 & 1.38 & 1.22 & 1.25 \\
\hline
\end{tabular}


Table 8: Results from rotational forced frequency excitation on the cart for the case where $\zeta=0.35$

\begin{tabular}{ccccc}
\hline$I=20 \mathrm{kgm}^{2}$ & $k=1000 \mathrm{Nm} / \mathrm{rad}$ & $c=100 \mathrm{Nsm} / \mathrm{rad}$ & $f_{n}=1.1 \mathrm{rad} / \mathrm{s}$ & $\zeta=0.35$ \\
\hline $\begin{array}{c}\text { Forced Freq. } \\
\mathrm{rad} / \mathrm{s}\end{array}$ & $\begin{array}{c}\text { Freq. Ratio } \\
\text { Simulated }\end{array}$ & $\begin{array}{c}\text { Freq. Ratio } \\
\text { Calculated }\end{array}$ & $\begin{array}{c}\text { Amp. Ratio } \\
\text { Simulated }\end{array}$ & $\begin{array}{c}\text { Amp. Ratio } \\
\text { Calculated }\end{array}$ \\
\hline 0.8 & 0.69 & 0.73 & 1.24 & 1.3 \\
1.0 & 0.88 & 0.92 & 1.18 & 1.2 \\
1.4 & 1.25 & 1.27 & 0.98 & 1.0 \\
1.8 & 1.60 & 1.64 & 0.53 & 0.60 \\
2.2 & 1.96 & 2.00 & 0.31 & 0.35 \\
\hline
\end{tabular}

Table 9: Results from rotational forced frequency excitation on the cart for the case where $\zeta=0.5$

\begin{tabular}{ccccc}
\hline$I=20 \mathrm{kgm}^{2}$ & $k=2000 \mathrm{Nm} / \mathrm{rad}$ & $c=200 \mathrm{Nsm} / \mathrm{rad}$ & $f_{n}=1.6 \mathrm{rad} / \mathrm{s}$ & $\zeta=0.5$ \\
\hline $\begin{array}{c}\text { Forced Freq. } \\
\mathrm{rad} / \mathrm{s}\end{array}$ & $\begin{array}{c}\text { Freq. Ratio } \\
\text { Simulated }\end{array}$ & $\begin{array}{c}\text { Freq. Ratio } \\
\text { Calculated }\end{array}$ & $\begin{array}{c}\text { Amp. Ratio } \\
\text { Simulated }\end{array}$ & $\begin{array}{c}\text { Amp. Ratio } \\
\text { Calculated }\end{array}$ \\
\hline 0.8 & 0.53 & 0.50 & 1.15 & 1.1 \\
1.0 & 0.64 & 0.63 & 1.22 & 1.2 \\
1.4 & 0.92 & 0.88 & 1.27 & 1.25 \\
1.8 & 1.14 & 1.13 & 0.88 & 0.90 \\
2.2 & 1.39 & 1.38 & 0.60 & 0.65 \\
\hline
\end{tabular}


Table 10: Results from rotational forced frequency excitation on the cart for the case where $\zeta=0.25$

\begin{tabular}{ccccc}
\hline$I=20 \mathrm{kgm}^{2}$ & $k=2000 \mathrm{Nm} / \mathrm{rad}$ & $c=100 \mathrm{Nsm} / \mathrm{rad}$ & $f_{n}=1.6 \mathrm{rad} / \mathrm{s}$ & $\zeta=0.25$ \\
\hline $\begin{array}{c}\text { Forced Freq. } \\
\mathrm{rad} / \mathrm{s}\end{array}$ & $\begin{array}{c}\text { Freq. Ratio } \\
\text { Simulated }\end{array}$ & $\begin{array}{c}\text { Freq. Ratio } \\
\text { Calculated }\end{array}$ & $\begin{array}{c}\text { Amp. Ratio } \\
\text { Simulated }\end{array}$ & $\begin{array}{c}\text { Amp. Ratio } \\
\text { Calculated }\end{array}$ \\
\hline 0.8 & 0.47 & 0.50 & 1.22 & 1.25 \\
1.0 & 0.64 & 0.63 & 1.57 & 1.55 \\
1.4 & 0.89 & 0.88 & 2.07 & 2.00 \\
1.8 & 1.10 & 1.13 & 1.88 & 1.90 \\
2.2 & 1.34 & 1.38 & 1.26 & 1.25 \\
\hline
\end{tabular}




\subsection{Validation of the Graham Model Through Ex- perimentation}

The Graham model was computationally validated with the inverted pendulum model, but it was also validated using physical apparatus specifically designed to represent the theoretical models. The experimental apparatus included a physical model of the inverted pendulum, the pendulating load model, the cart load model, and a rigid interface connector. The physical models were designed in order to be coupled or uncoupled using the interface connector. The experiments were performed on a sixdegree-of-freedom motion platform. The following section provides a brief overview of the design of the apparatus, the validation experiments to validate the Graham model, followed by the validation of the Graham model coupled to the pendulating and cart loads.

\subsubsection{Experimental Apparatus}

The mechanical hardware used for the validation experiments was developed for use on the PSM project contracted by DRDC Atlantic. The apparatus was designed and built as part of the broader PSM project. Also, as part of the broader PSM project validation of the inverted pendulum and pendulating load models developed by Langlois was also performed using the same apparatus. This section will outline the design of the apparatus pertinent to the models included in the report; the Graham stability and cart load models.

The scale of the apparatus was desired to be 1:1. This would allow the potential for future experimentation using human subjects for determining the effects of unsteady loads on human postural stability and for tuning the developed models. It was first required to determine the feasibility of this scaling. It was determined that at a 1:1 
scale the maximum mass that would be required on the stability models would be $200 \mathrm{lb}(90.7 \mathrm{~kg})$ and for the load models it would be $400 \mathrm{lb}(181.4 \mathrm{~kg})$. This weight requirement is not excessive relative to the motion platform maximum load and it was recognized that thirty-five pound weightlifting plates could be used at an acceptable cost. Since this apparatus was also needed to control the inverted pendulum model it was critical that the control motors be able to provide the required torques for stability and representative actuation. It was found that the maximum torque required would be $200 \mathrm{ft}-\mathrm{lb}(271.2 \mathrm{Nm})$ at the inverted pendulum rotation centre. This value was found through simulation of the inverted pendulum model for a severe ship motion case while coupled to a large pendulating load. The torques required in order to maintain the apparatus rigid for the representative Graham model would not be in excess of the torques required by the inverted pendulum in the test case. It was found that this torque was feasible with a geared servo-motor system. Based on these considerations and the available hardware budget it was decided to proceed with design of the system using the 1:1 scale.

\section{Graham Model Apparatus Design}

The design of the Graham model apparatus was coupled to the design of the inverted pendulum apparatus. Since the design was coupled, many of the more stringent design requirements for this apparatus came from the requirements of the inverted pendulum model. This section outlines the design of the apparatus that is used as the physical apparatus for validating both the inverted pendulum and Graham stability models.

To achieve the control torque of up to $200 \mathrm{ft}-\mathrm{lb}(271.2 \mathrm{Nm})$ required for the inverted pendulum model, Kollmorgen AKM-42E electric servo motors were paired with Apex Dynamics AE-120-100 and AER-120-100 gear sets as can be seen in Figure 35. 
These gear sets provide a large reduction ratio of 100:1 so that the rated motor output torque of $2.3 \mathrm{ft}-\mathrm{lb}(3.1 \mathrm{Nm})$ is sufficient. For the maximum anticipated pendulum rotational speed of 30 degrees per second, approximately 500 RPM motor speed is expected. This operating state is within the AKM-42E motor capability for continuous operation. The AER-120-100 gearbox, which includes a 90 degree elbow between gear stages, is required so that the drive system attached to the moving portion of the inverted pendulum universal joint does not strike the ground during motion.

The spatial inverted pendulum uses a unique form of control input. Unlike classical examples of inverted pendulums, control torque is given to a single rotation centre comprised of two intersecting, perpendicular axes of rotation. This feature is a requirement to accurately re-create the dynamic model of the spatial inverted pendulum with two degrees of rotational freedom. Such torque input is enabled using a custom-designed universal joint, shown in Figure 36. Two perpendicular rotation pins are machined to interface with the keyed output shafts of the AE-120 and AER120 gear sets. Torque from the gear sets is then applied to the rotation centre of the inverted pendulum through the two intersecting perpendicular axes of these rotation pins. A custom $\mathrm{U}$-joint was required for this application to accommodate the large diameters of the AE-120 and AER-120 gear set output shafts. The U-joint component used four off-the-shelf ball bearings and laser-cut mild steel structures. Components were joined using MIG welding. The U-joint was installed on two laser-cut steel discs with hole patterns that allow the inverted pendulum base to be rotated in 15 degree increments relative to the base structure on which it is mounted.

The spatial inverted pendulum, shown in Figure 35, was completed using 1.5" $(3.81 \mathrm{~cm})$ schedule 40 steel pipe affixed atop the U-joint to rigidly hold the $35 \mathrm{lb}$ $(15.9 \mathrm{~kg})$ mass plates at a desirable distance from the rotation centre. Up to five mass plates can be accommodated at any time.

For use of the apparatus for the Graham stability model, there is an angled locking 


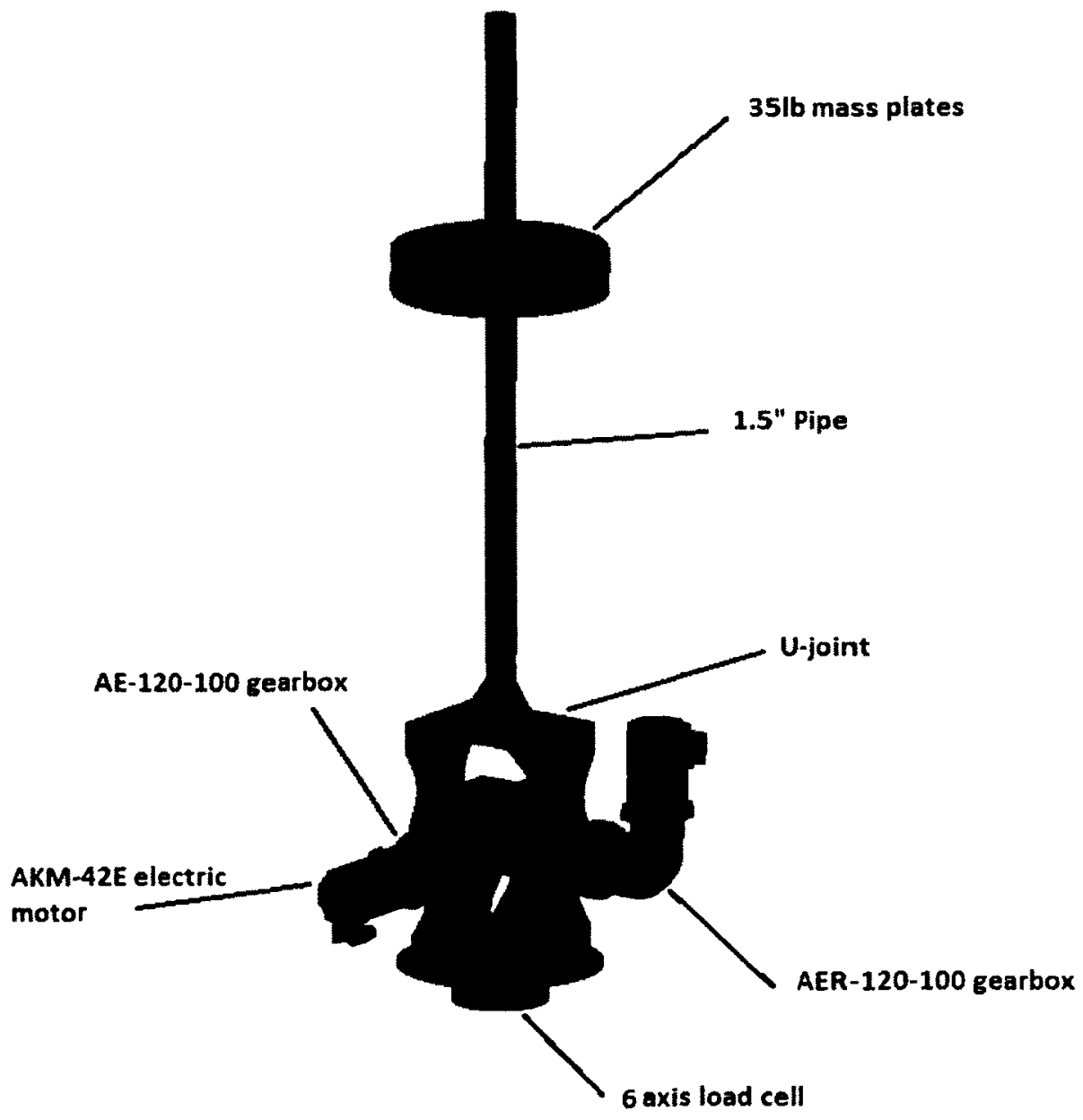

Figure 35: Spatial inverted pendulum physical model. 


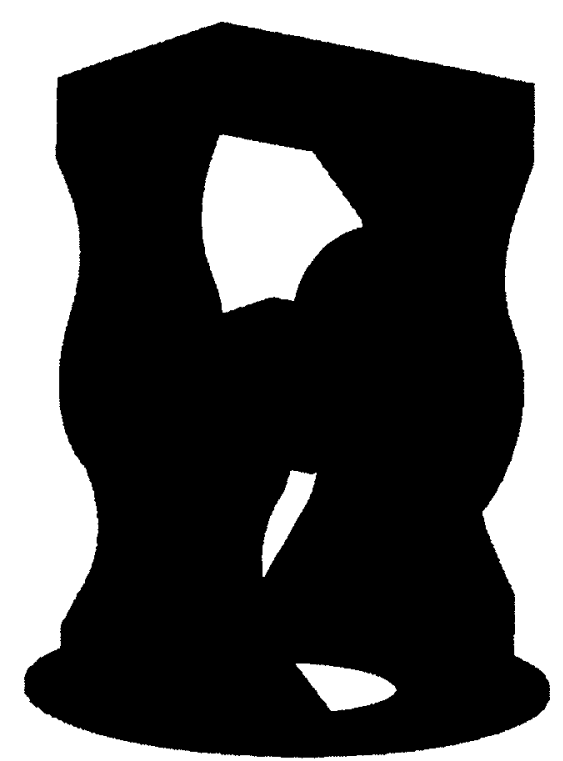

Figure 36: Inverted pendulum custom-designed universal joint.

plate made out of mild steel. The steel plate is attached to the two faces of the U-joint opposite the gear boxes and motors. The locking plate once attached stops rotation through the $U$-joint causing the inverted pendulum to act in the same way as the rigid body Graham model.

\section{Cart Load Apparatus Design}

The cart load hardware consists of two moving components: a translating base and a rotational structure mounted atop the translating base. The intent of this design is to facilitate reconfiguration of the hardware to accommodate various motion states including single degree of freedom translation or combined translation and rotation. The translating base, shown in Figure 37, travels within two guide rails comprised of structural steel C-channel and off-the-shelf roller guides. The C-channel is sized to provide adequate stiffness of the support structure. 


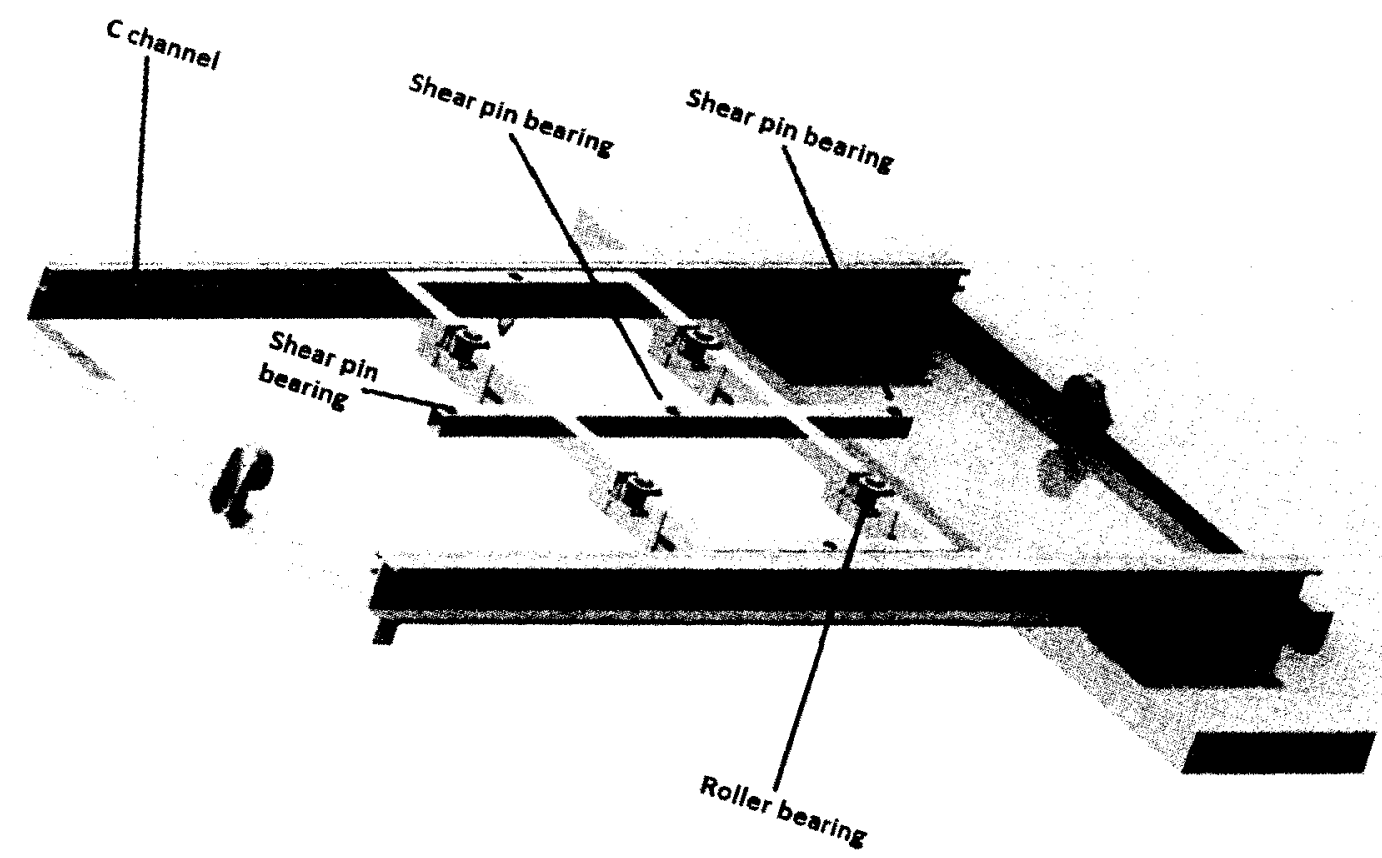

$$
\text { Figure 37: Cart load translating } b_{\text {ase }} \text { design. }_{\text {. }}
$$




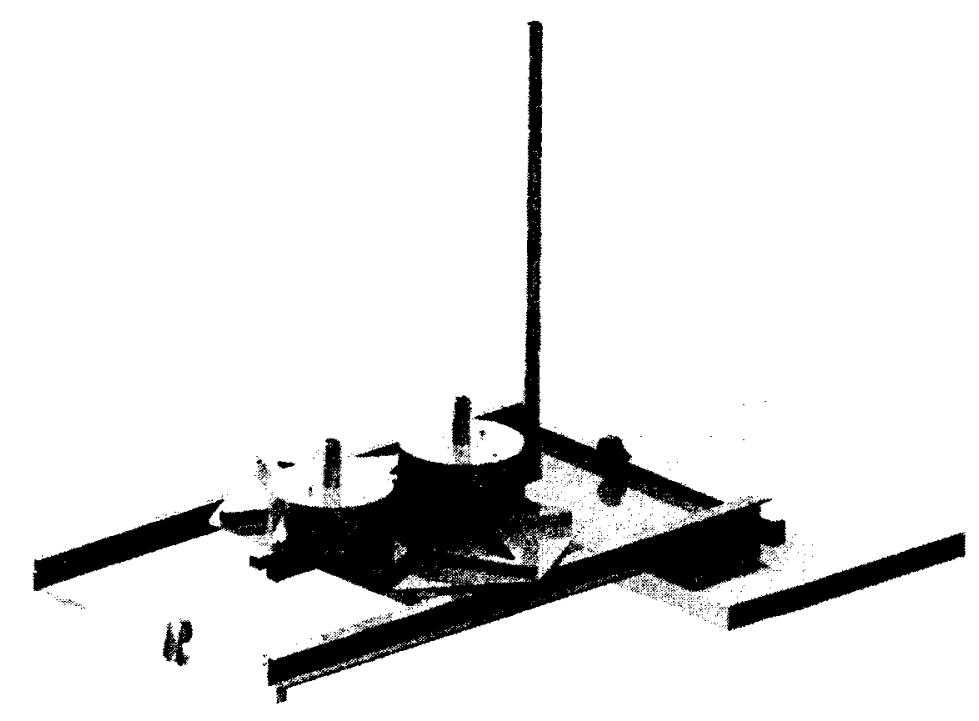

Figure 38: Solid model of the assembled cart load arrangement.

Translation of the rotational structure relative to the translating base is constrained by ball bearings and shear pins, also seen in Figure 37. By installing one shear pin, rotation is allowed about its axis. The shear pin can be installed in any one of three bearing locations to allow reconfigurable rotation centres for the cart load. Rotation is prevented when shear pins are installed in any two of the bearing locations. The rotational structure is supported by four ball transfers that act on a large laser cut steel sheet. This sheet is backed by a steel frame structure designed to cover the expected path of the ball transfers to the greatest extent possible.

A section of $1.5 "(3.81 \mathrm{~cm})$ schedule 40 steel pipe is mounted at one end of the rotational structure, serving as a location where interface forces can be applied. The rotational structure is also the mounting location for up to eleven $35 \mathrm{lb}(15.9 \mathrm{~kg})$ mass plates. Figure 38 shows the assembled cart load hardware. 


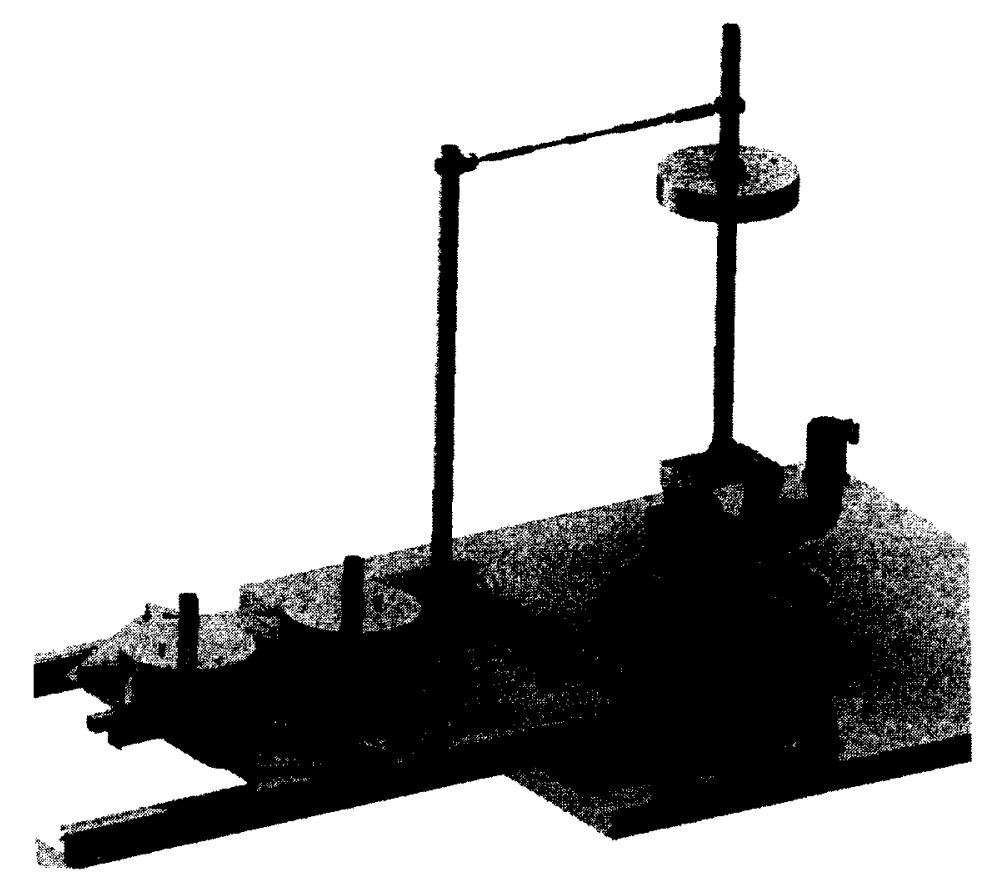

Figure 39: Implementation of the tension/compression link with the cart load model.

\section{Interface Connection Apparatus Design}

Interface forces between the actively-controlled spatial inverted pendulum and the dynamic load hardware are transferred via a rigid tension/compression link. The link is made of steel pipe segments and a combination of right and left-handed threaded rods for simple length adjustment. The link connects to the inverted pendulum and dynamic load hardware using steel set screw collars which fasten to the $1.5 "(3.81 \mathrm{~cm})$ steel pipe used in all three primary hardware systems. To allow the necessary degrees of freedom between the inverted pendulum and dynamic load systems, a universal joint connects the steel pipe to one of the set screw collars while a spherical bearing rod-end connects the other. These connections allow sufficient degrees of freedom to obtain a link which is subjected only to tensile or compressive forces. Figure 39 shows how the tension/compression link is implemented to connect the spatial inverted pendulum to the cart load. 
Table 11: Simulation parameters used with GRM3D and PSM3D

\begin{tabular}{cc}
\hline Parameter & Value \\
\hline$m$ & $63.7 \mathrm{~kg}$ \\
$I_{x x}$ & $78555 \mathrm{~kg} \mathrm{~m}^{2}$ \\
$I_{y y}$ & $777746 \mathrm{~kg} \mathrm{~m}^{2}$ \\
$I_{z z}$ & $6060 \mathrm{~kg} \mathrm{~m}^{2}$ \\
$\mathbf{r}_{C G / A}^{M O}$ & $\{-0.60 .340 .75\}^{T} \mathrm{~m}$ \\
$\mathrm{~g}$ & $9.81 \mathrm{~m} / \mathrm{sec}^{2}$ \\
\hline
\end{tabular}

\subsubsection{Experimental Validation of the Graham Stability Model}

The main results from the Graham stability model were the reaction forces and moments acting at the base of the model. The test cases are designed to isolate these variables and incrementally increase the degrees of freedom being validated. For the experimentation, the weight added to the apparatus was $70 \mathrm{lbf}(31.75 \mathrm{~kg})$. The parameters of the physical model were calculated from the Pro Engineer CAD model used to design the hardware. The physical parameters were calculated within the program and are provided in Table 11

To isolate a translational acceleration, a constant frequency sinusoidal sway motion was used. This motion profile allowed for isolation of a translational degree of freedom, as well as providing a smooth acceleration profile to the model. In order to remain within the motion platform performance limitations, the amplitude of the motion was selected to be $0.1 \mathrm{~m}$ with a frequency of $0.5 \mathrm{rad} / \mathrm{sec}$. A second motion profile isolated an angular degree of freedom by using a constant frequency sinusoidal roll motion. The roll motion had an amplitude of $0.1 \mathrm{rad}$ and a frequency of $0.5 \mathrm{rad} / \mathrm{sec}$ 
in order to remain within the platform motion limits. Following the constant frequency sinusoidal motion, a frequency sweep sinusoidal motion was performed. This motion had the advantage of determining the frequency response of the system. The frequency sweep was performed for both a single degree of freedom translational and angular motion in the sway and roll directions respectively. The frequency swept from $0.1 \mathrm{rad} / \mathrm{sec}$ through $0.5 \mathrm{rad} / \mathrm{sec}$. The final validation motion profile for the stability models was a six degree of freedom representative ship motion. This general motion case was used to validate the model for all six degrees of freedom to ensure the results were acceptable. The ship motion was generated based on representative frigate and sea state data.

The results from the physical apparatus were the forces and moments at the base measured using the load cell. The load cell was sampling results at $100 \mathrm{~Hz}$ and had high frequency noise. The noise was filtered out using a fourth-order two-pass Butterworth filter. Additional post processing of the data was needed in order to line up the experimental coordinate frames with the simulation coordinate frames. The experimental coordinate frames can be seen in Figure 40. In order to compare the two sets of results the experimental results were rotated to the dynamic model frame. First to note is that the motion platform does not have the same coordinate system as the dynamic model. This means that the input motions needed to be rotated to the dynamic coordinate system. That required a simple transformation matrix

$$
\left[T_{\text {DynamicModel }}^{\text {Platform }}\right]=\left[\begin{array}{ccc}
1 & 0 & 0 \\
0 & -1 & 0 \\
0 & 0 & 1
\end{array}\right]
$$

A similar transformation matrix can be used to rotate the forces from the load cell to the dynamic model coordinate frame. The angle between the dynamic model 

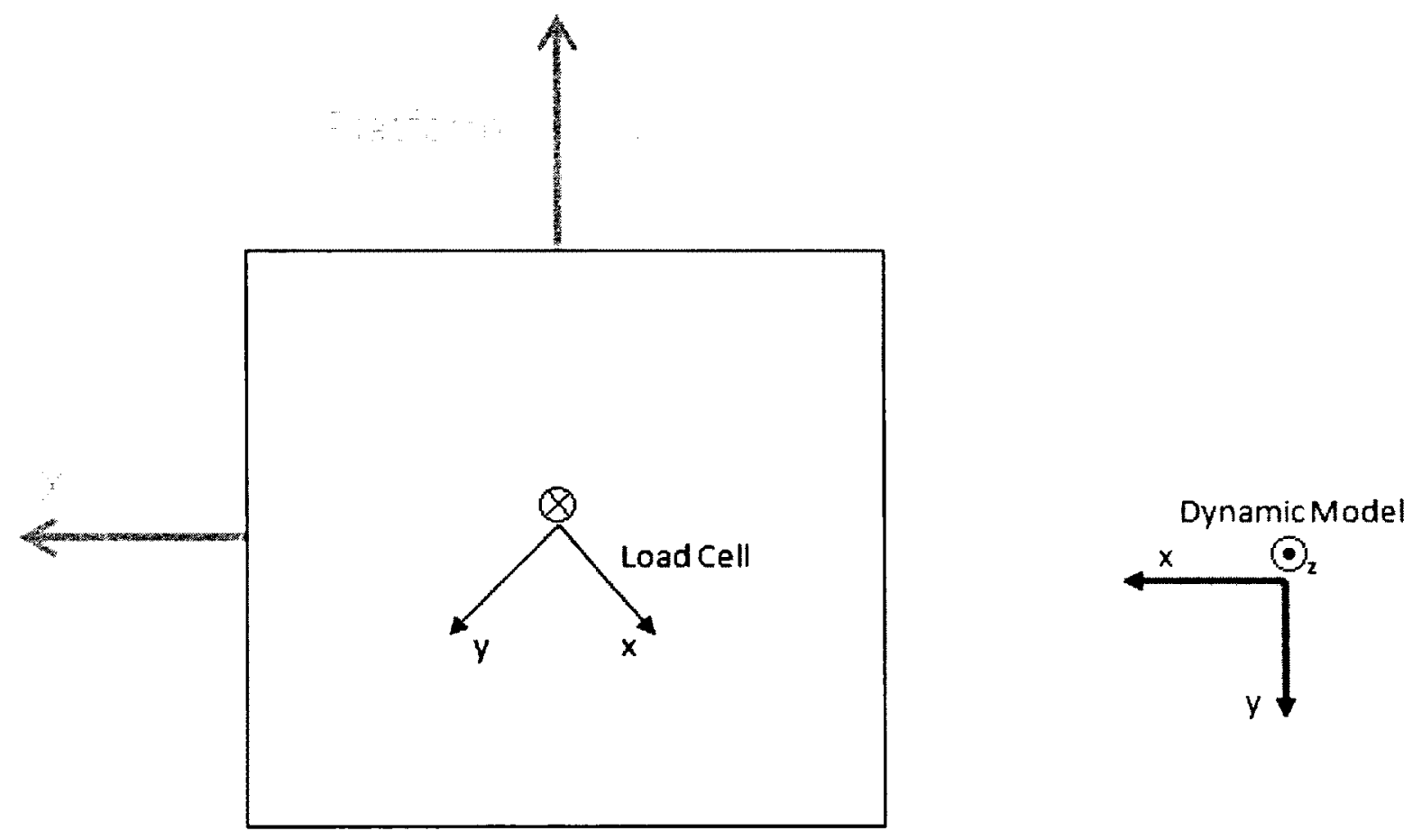

Figure 40: Coordinate systems for the experimental apparatus compared to the dynamic model.

and the load cell frames was approximately measured as 45 degrees

$$
\left[T_{\text {DynamicModel }}^{\text {LoadCell }}\right]=\left[\begin{array}{ccc}
\sin 45^{\circ} & \cos 45^{\circ} & 0 \\
\cos 45^{\circ} & -\sin 45^{\circ} & 0 \\
0 & 0 & -1
\end{array}\right]
$$

Finally, the moments measured from the load cell had to be rotated to the dynamic model frame. Since the moment results from a cross product, the vector cannot be simply rotated from one coordinate frame to the other like a polar vector, so the moment was broken down into the force and position vectors and then rotated. Consider the example seen in Figure 41, it can be seen that a force and a moment 


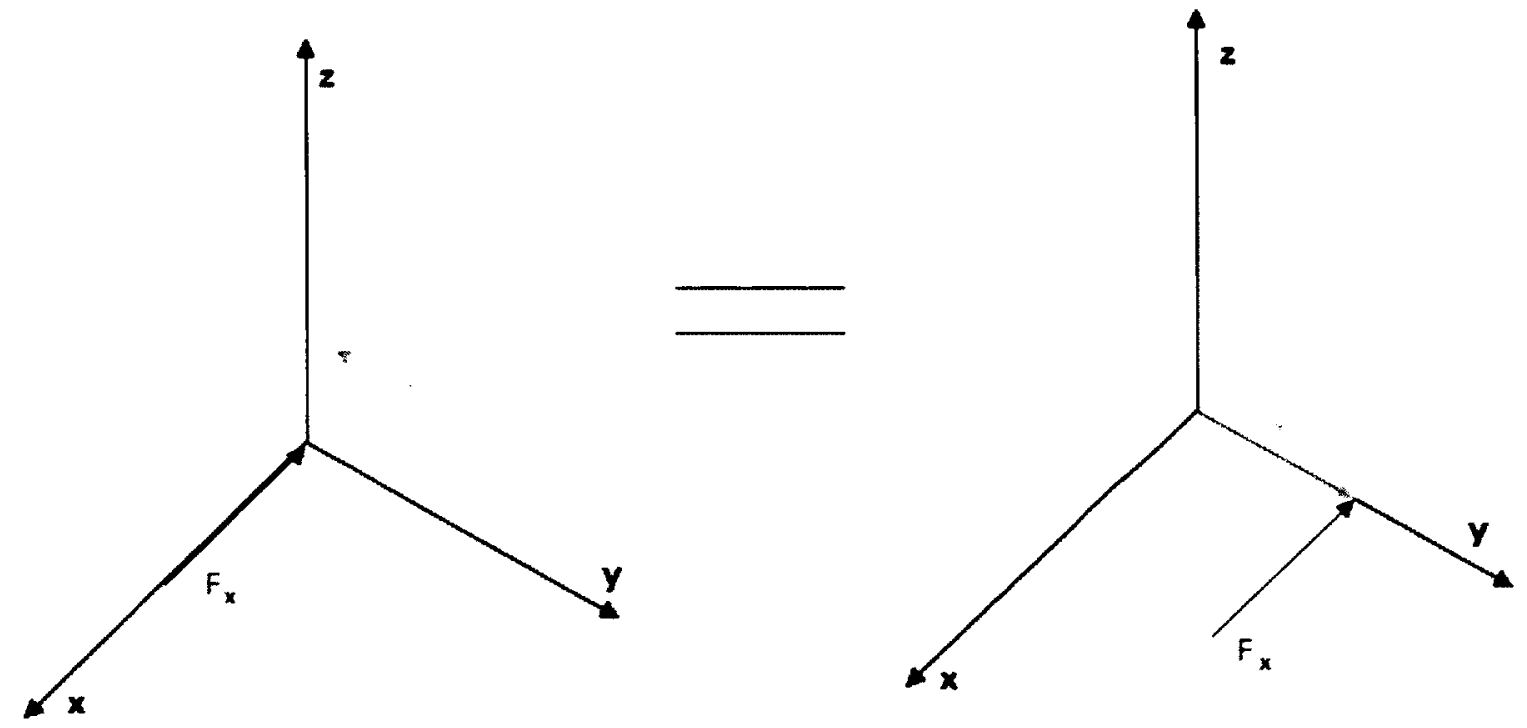

Figure 41: Example of breaking moment into force and position vector components.

experienced is equivalent if it was assumed that the force was being applied at a distance along the axis. From the measurements, the force magnitude was known, and therefore the distance along the axis was calculated.

$$
\left|r_{y}\right|=\frac{\left|M_{z}\right|}{\left|F_{x}\right|}
$$

The direction of the force vector is known to be along the $x$ axis of the load cell coordinate frame. The definition of a moment is the cross product of the position vector with the force vector. Assuming that the load cell coordinate frame is $A$ and the dynamic model coordinate frame is $B$, the moment about the $z$ axis is

$$
\vec{M}_{z}^{A}=\vec{r}_{y}^{A} \times \vec{F}_{x}^{A}
$$

For the moment to be expressed in coordinate system $B$, the position and force vectors must be rotated 


$$
\vec{M}_{z}^{B}=R_{B}^{A} \vec{r}_{y}^{A} \times R_{B}^{A} \vec{F}_{x}^{A}
$$

Using the expression in Equation 72 and the rotation matrix defined in Equation 69 the moments measured from the load cell were rotated in order to be compared to the result from the simulation.

The results for the Graham model simulation and experimental results for the general ship motion case can be seen in Figures 42 through 47 . It can be seen from the graphs that the forces and moments calculated from the simulation are similar to those collected from the experimentation.

\subsubsection{Validation of the Coupled Graham Model with the Pendulum and Cart Loads through Experimentation}

Once the validation of the individual models was completed, the models were coupled using the tension and compression link and therefore needed to be validated again in this coupled operating mode with the simulation results. The simulation model allowed for the coupling of the Graham model with either the pendulum or cart load models, resulting in two different configurations that needed to be validated. Both of the configurations were validated with the same input motions.

The parameters used for validating the coupled models were similar to those used to validate the models separately. The motion inputs used to validate the coupled models were linear sinusoidal sway motion with an amplitude $0.1 \mathrm{~m}$ and a frequency of $0.5 \mathrm{rad} / \mathrm{sec}$. A second motion profile was used to isolate an angular degree of freedom and was a constant frequency sinusoidal roll with an amplitude of 0.1 rad and a frequency of $0.5 \mathrm{rad} / \mathrm{sec}$. To validate the model for all six degrees of freedom, ship motion was generated based on typical frigate and sea state data.

These coupled models were run for two different weights: $0 \mathrm{lbs}(0 \mathrm{~kg})$ and $70 \mathrm{lbs}$ 


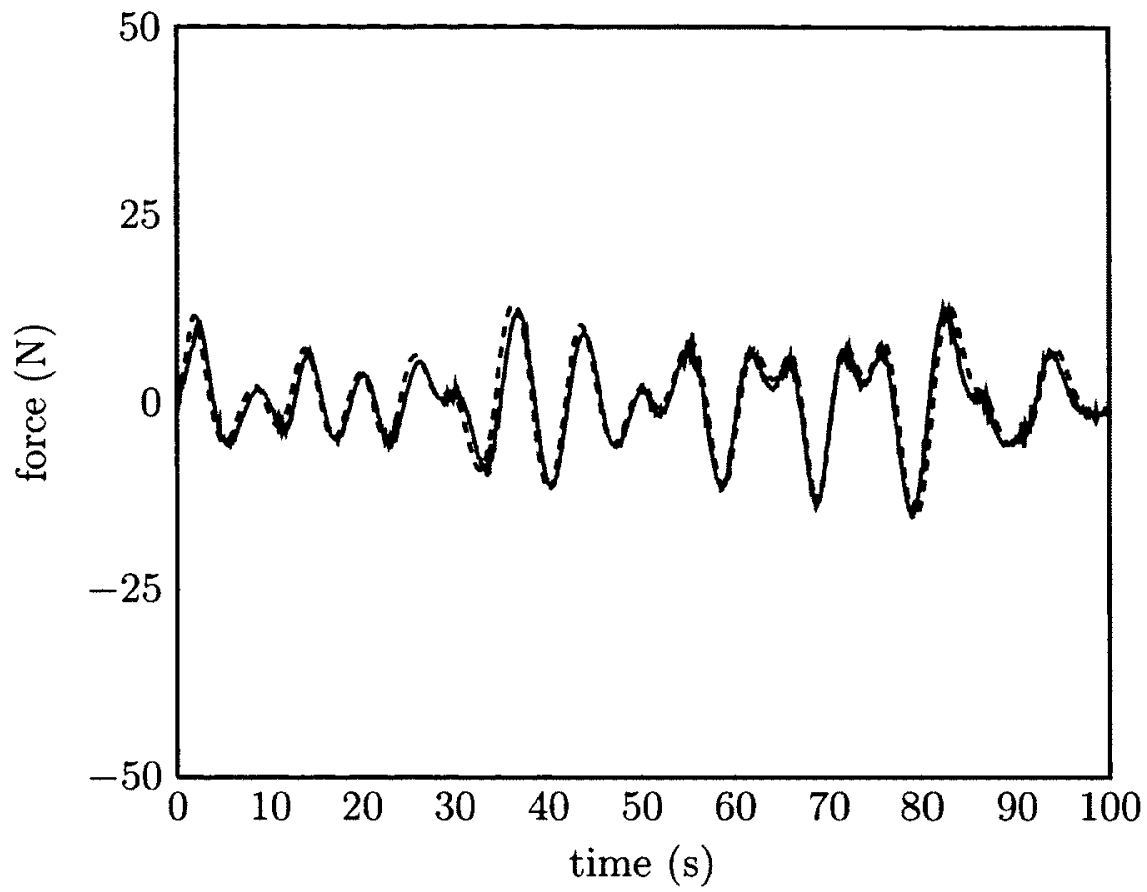

Figure 42: Longitudinal force $\left(F_{x}\right)$ computed using GRM3D (solid) and the load cell results from the experimentation (dashed).

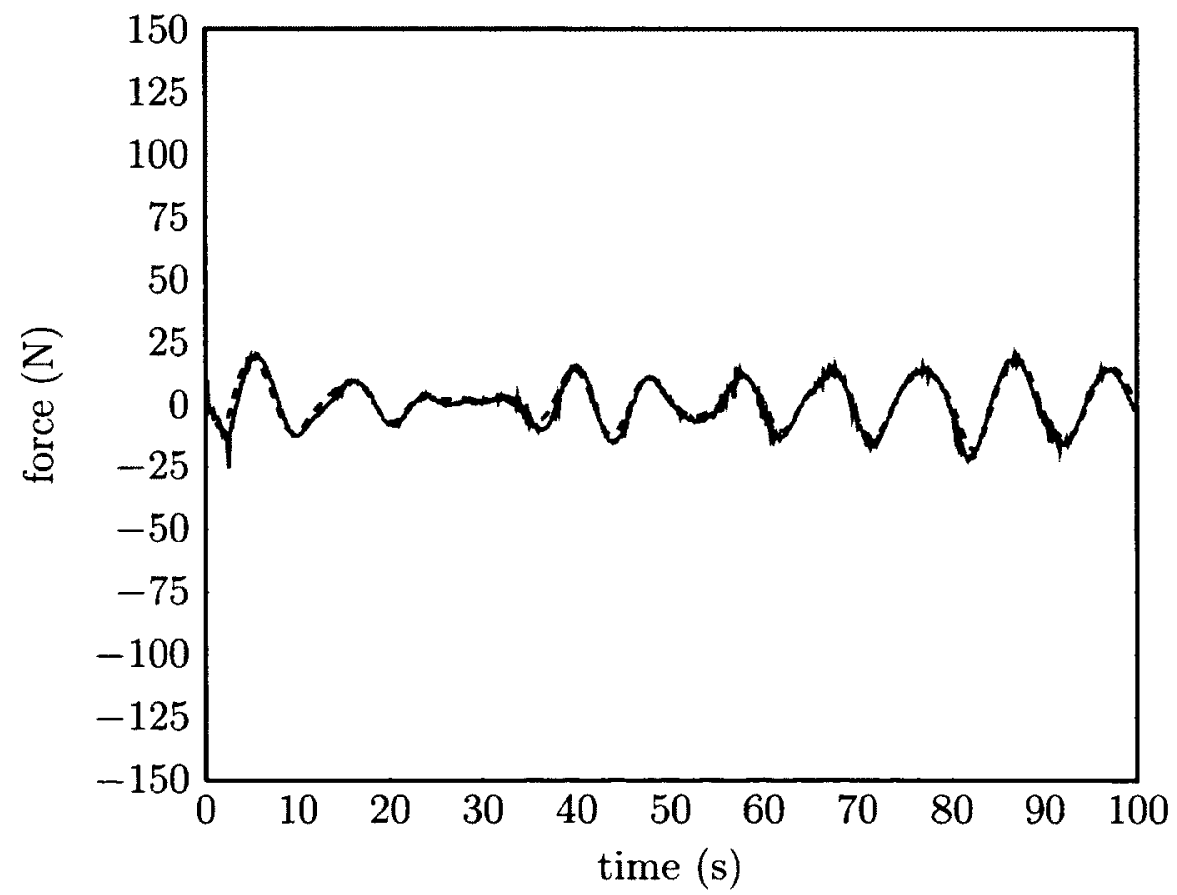

Figure 43: Lateral force $\left(F_{y}\right)$ computed using GRM3D (solid) and the load cell results from the experimentation (dashed). 


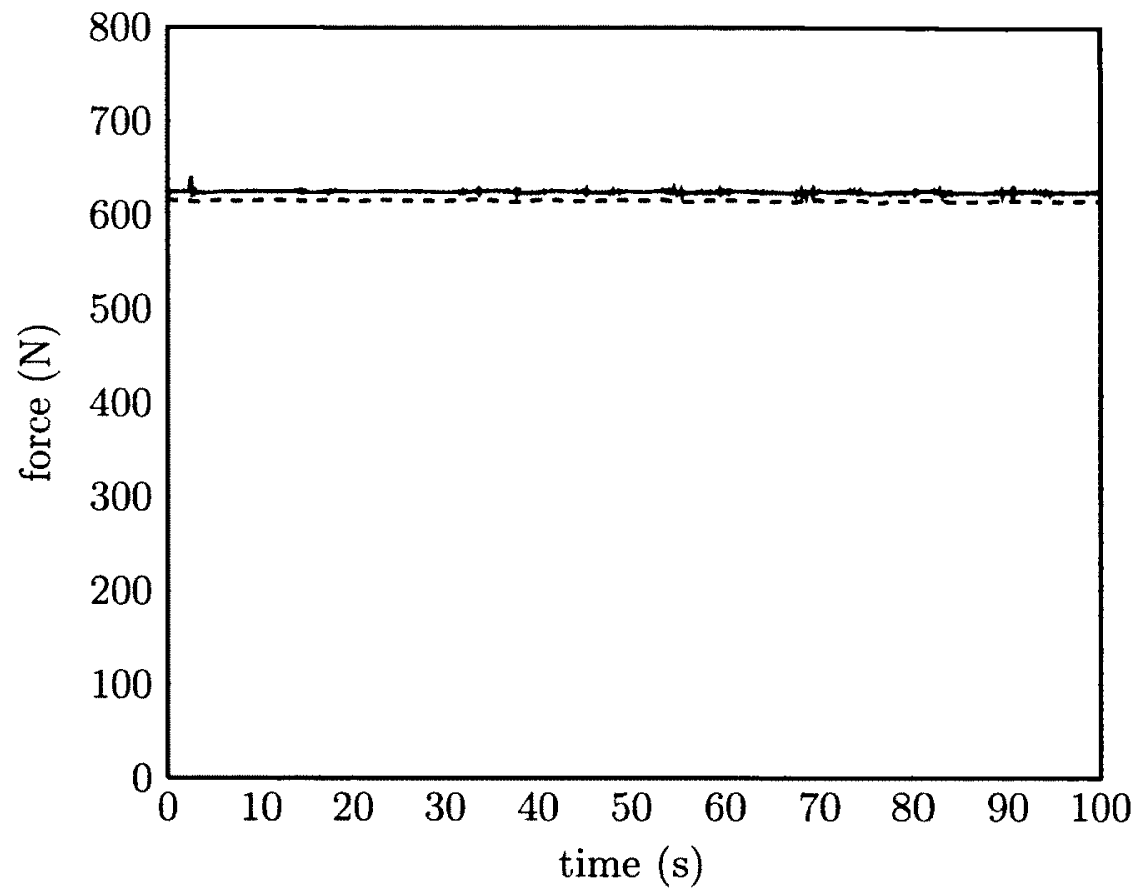

Figure 44: Vertical force $\left(F_{z}\right)$ computed using GRM3D (solid) and the load cell results from the experimentation (dashed).

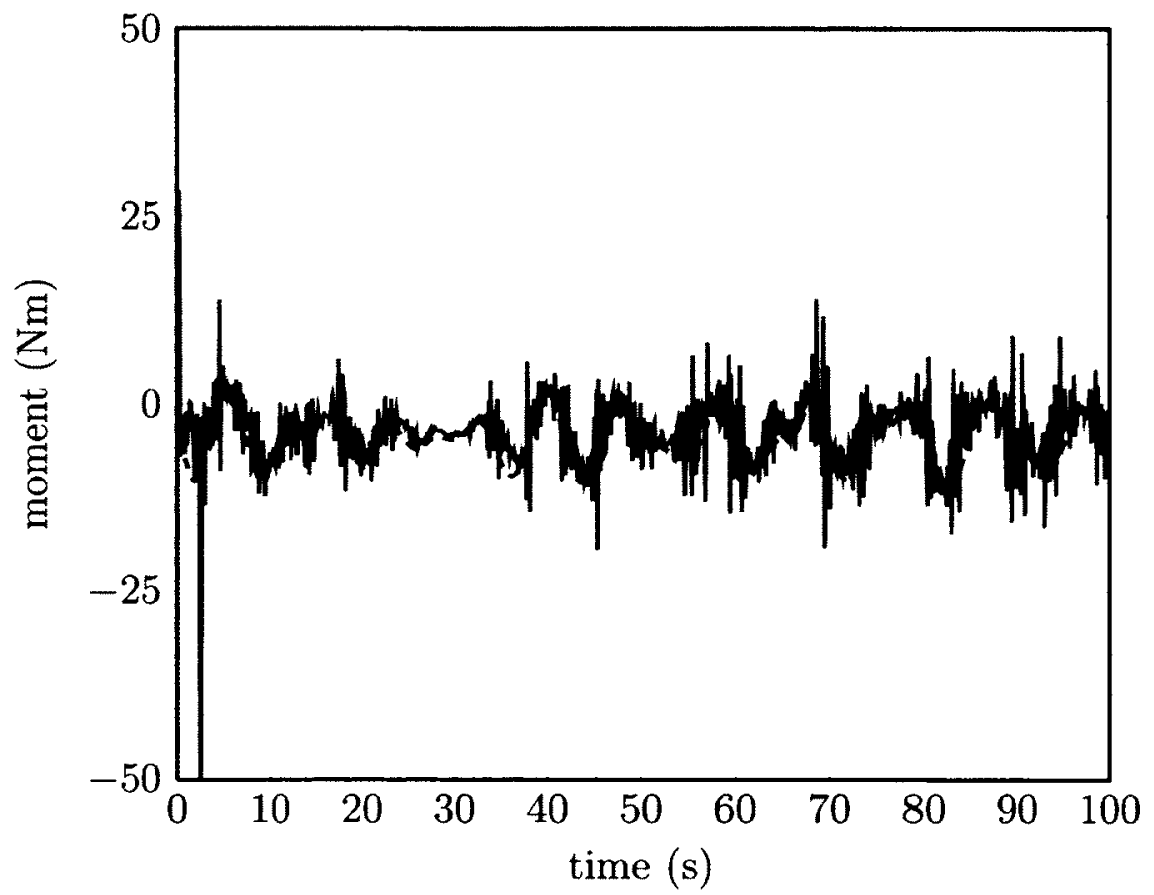

Figure 45: Longitudinal moment $\left(M_{x}\right)$ computed using GRM3D (solid) and the load cell results from the experimentation (dashed). 


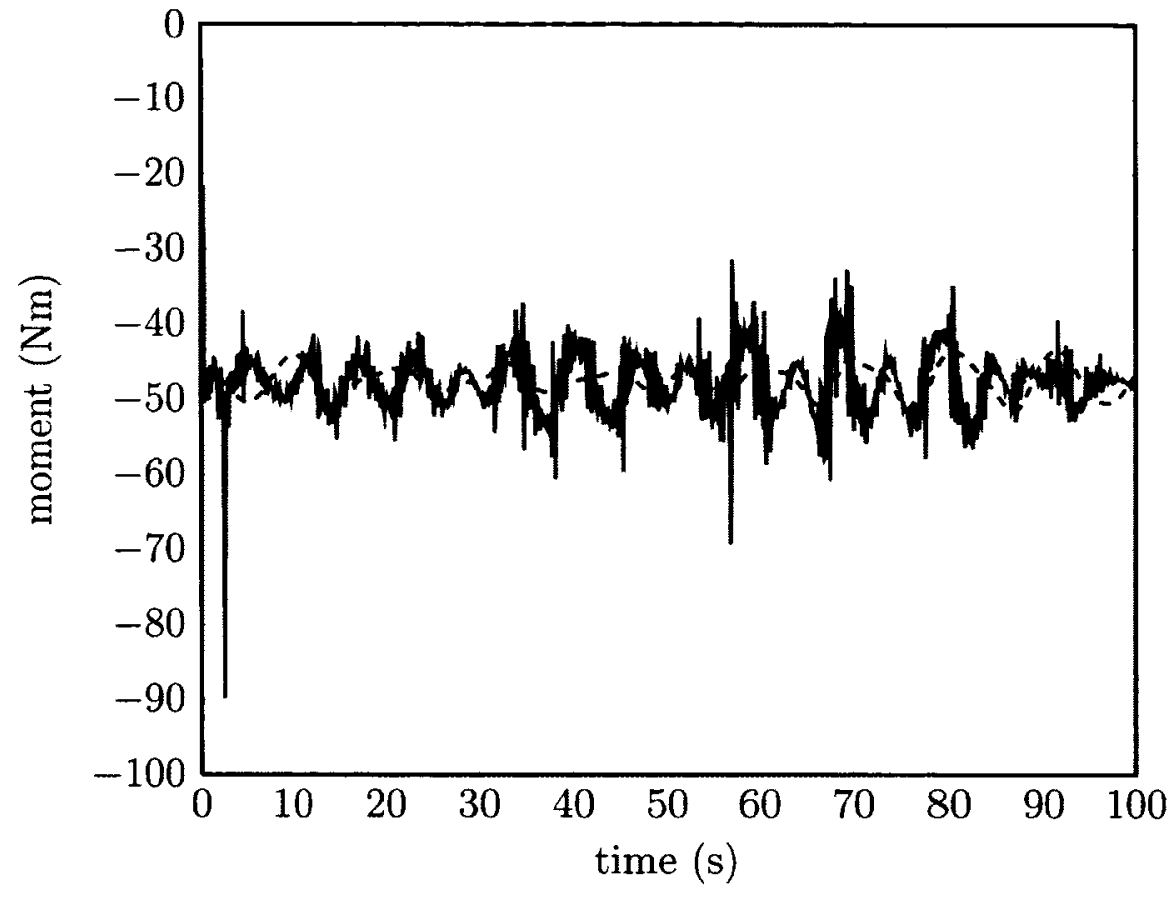

Figure 46: Lateral moment $\left(M_{y}\right)$ computed using GRM3D (solid) and the load cell results from the experimentation (dashed).

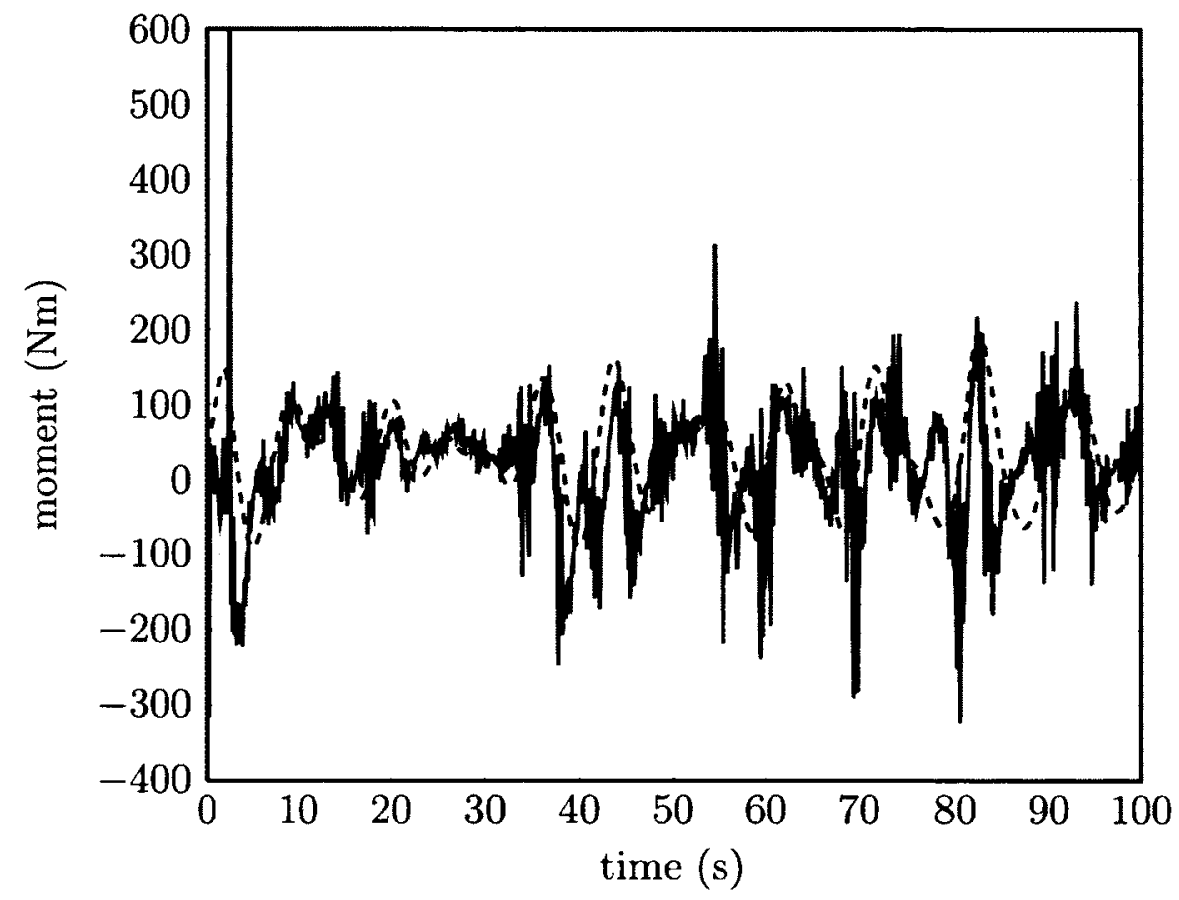

Figure 47: Vertical moment $\left(M_{z}\right)$ computed using GRM3D (solid) and the load cell results from the experimentation (dashed). 
$(31.75 \mathrm{~kg})$, so that there were $1: 1,1: 2$, and $2: 1$ stability model to load model ratios. This allowed for more comprehensive validation using the different weights, which might have different effects on the physical models. Also, for the cart model, the point of rotation of the top mass could be altered, and two rotation configurations were investigated. The physical parameters used for the Graham model are the same as previously stated and provided in Table 11.

The results from the experimentation have been compared to the simulations. Sample results can be seen in Figures 48 through 53 that correspond to the general ship motion case with no additional mass plates on the stability model and two mass plates on the pendulum load model. Figures 54 through 59 correspond to the general motion case with no additional mass plates on the stability model and no mass plates on the cart load model. The experimental data have been filtered using a fourthorder two-pass Butterworth filter to remove high frequency noise. The simulation data has been transformed from the experimental to the simulation coordinate frame. The results from both the simulation and experimentation are similar. The results show that the coupled models have been validated for the general ship motion case. The results for the longitudinal and lateral forces with the pendulum load attached showed variations from the experimental trends. This may be due to an error caused by the rotation from the experimental to simulation frames. Also, the case with the cart load attached the results from the simulation for the lateral moment showed variations from the experimental trends. 


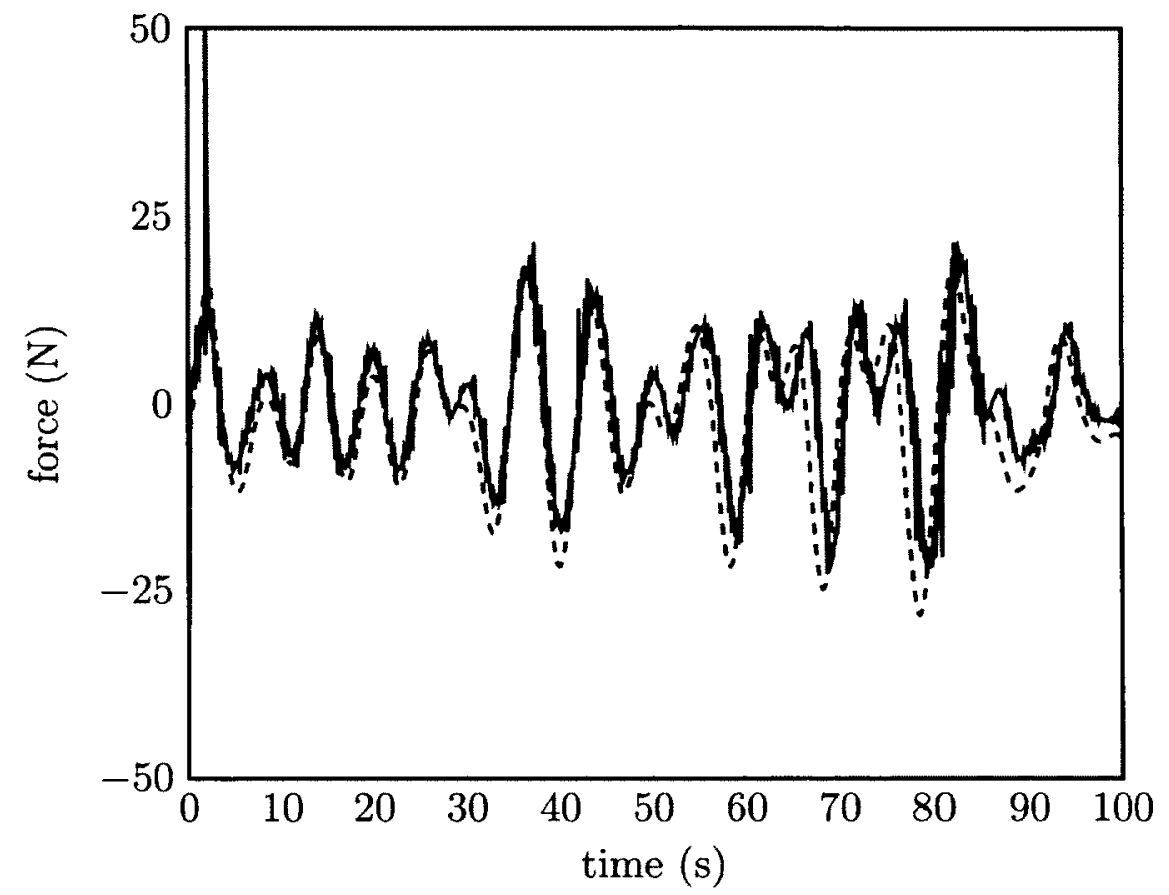

Figure 48: Longitudinal force $\left(F_{x}\right)$ computed using GRM3D (solid) coupled to pendulum load and the load cell results from the experimentation (dashed).

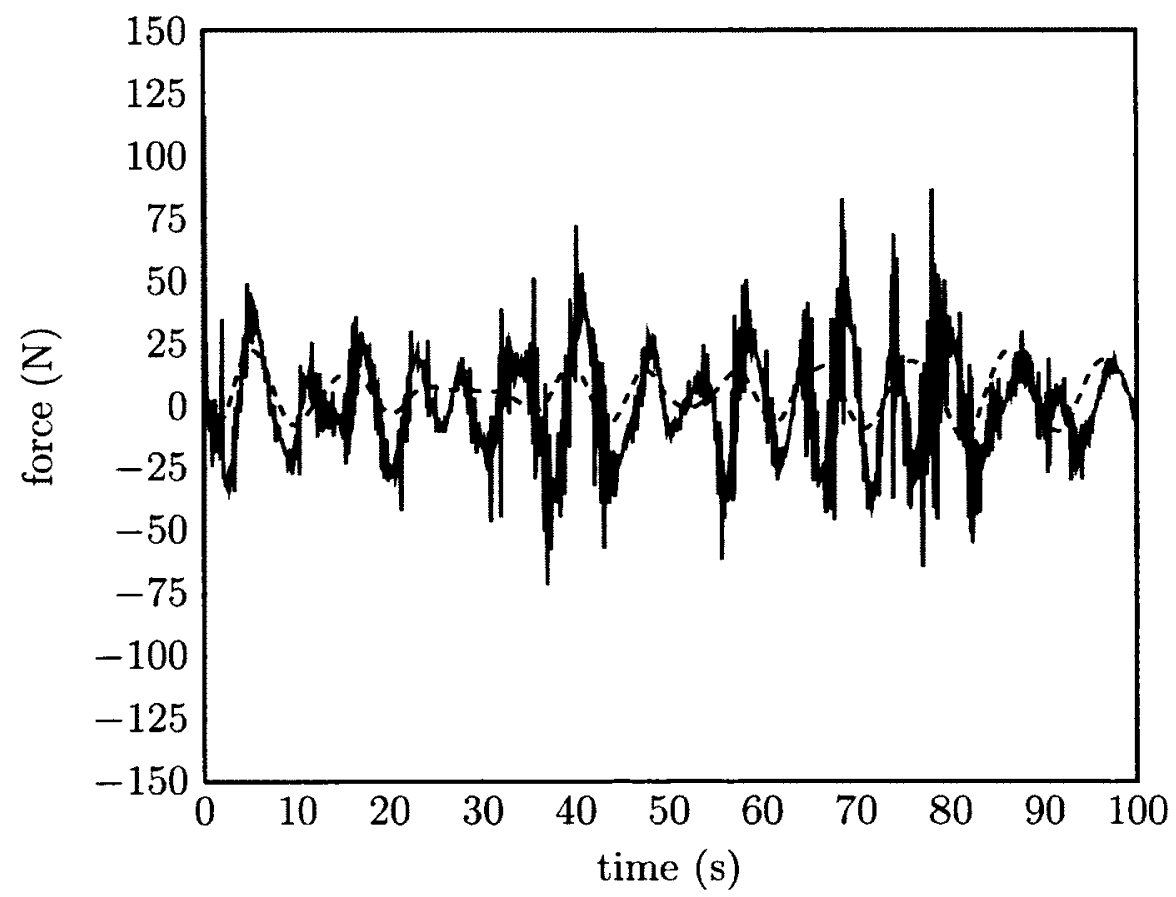

Figure 49: Lateral force $\left(F_{y}\right)$ computed using GRM3D (solid) coupled to the pendulum load and the load cell results from the experimentation (dashed). 


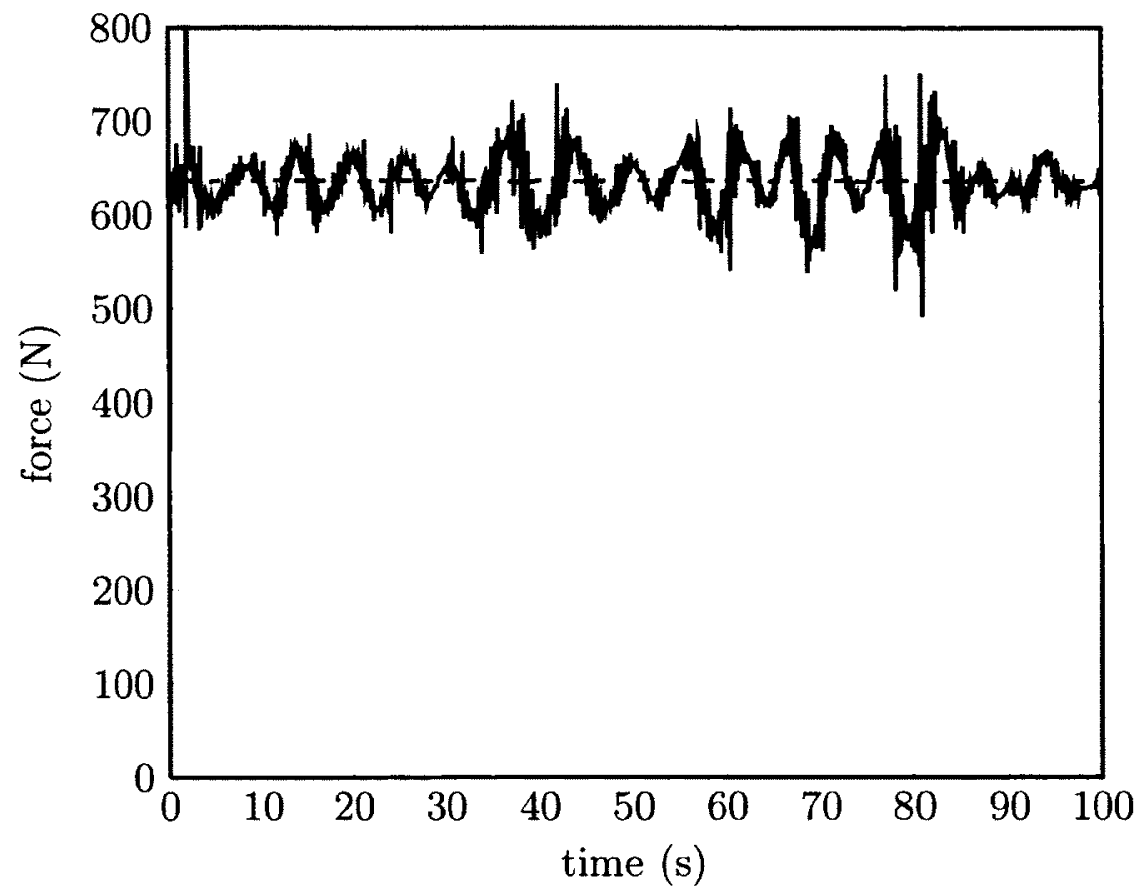

Figure 50: Vertical force $\left(F_{z}\right)$ computed using GRM3D (solid) coupled to pendulum load and the load cell results from the experimentation (dashed).

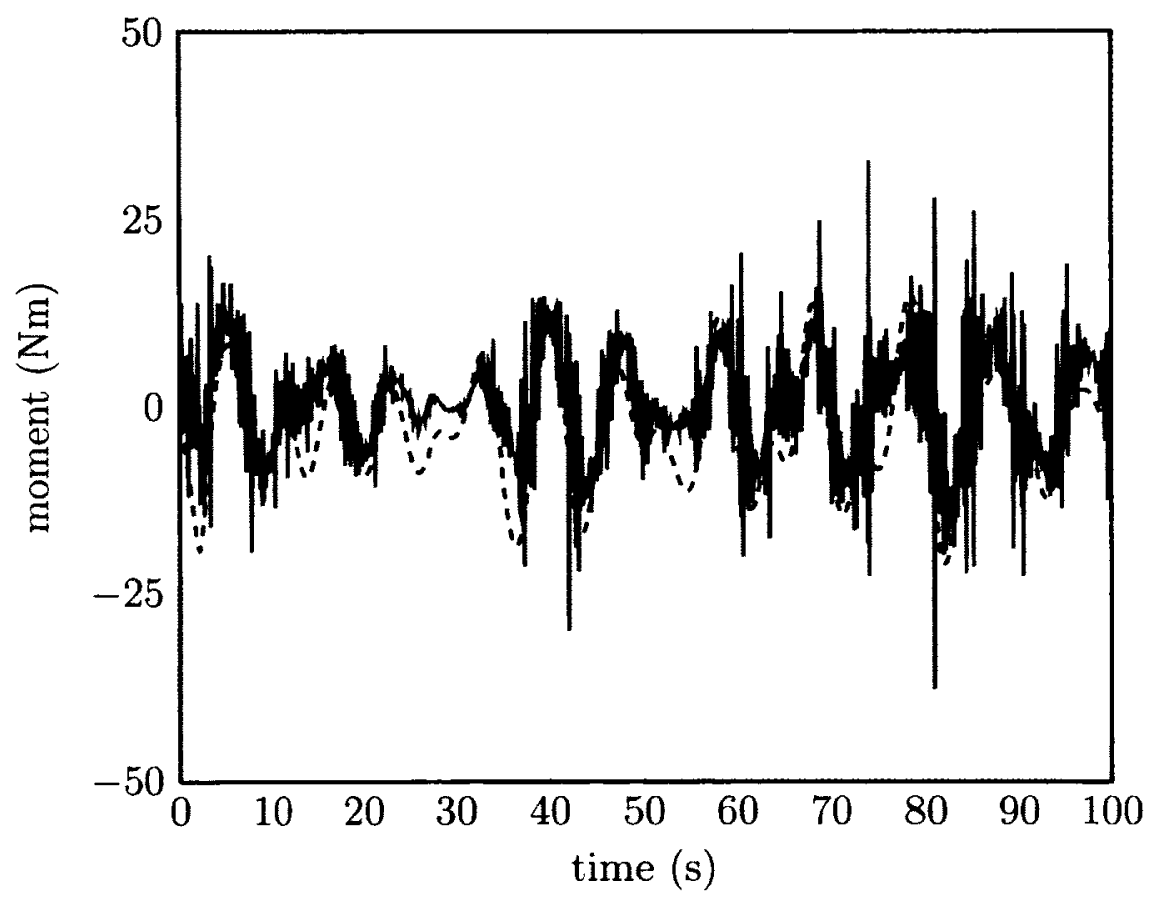

Figure 51: Longitudinal moment $\left(M_{x}\right)$ computed using GRM3D (solid) coupled to pendulum load and the load cell results from the experimentation (dashed). 


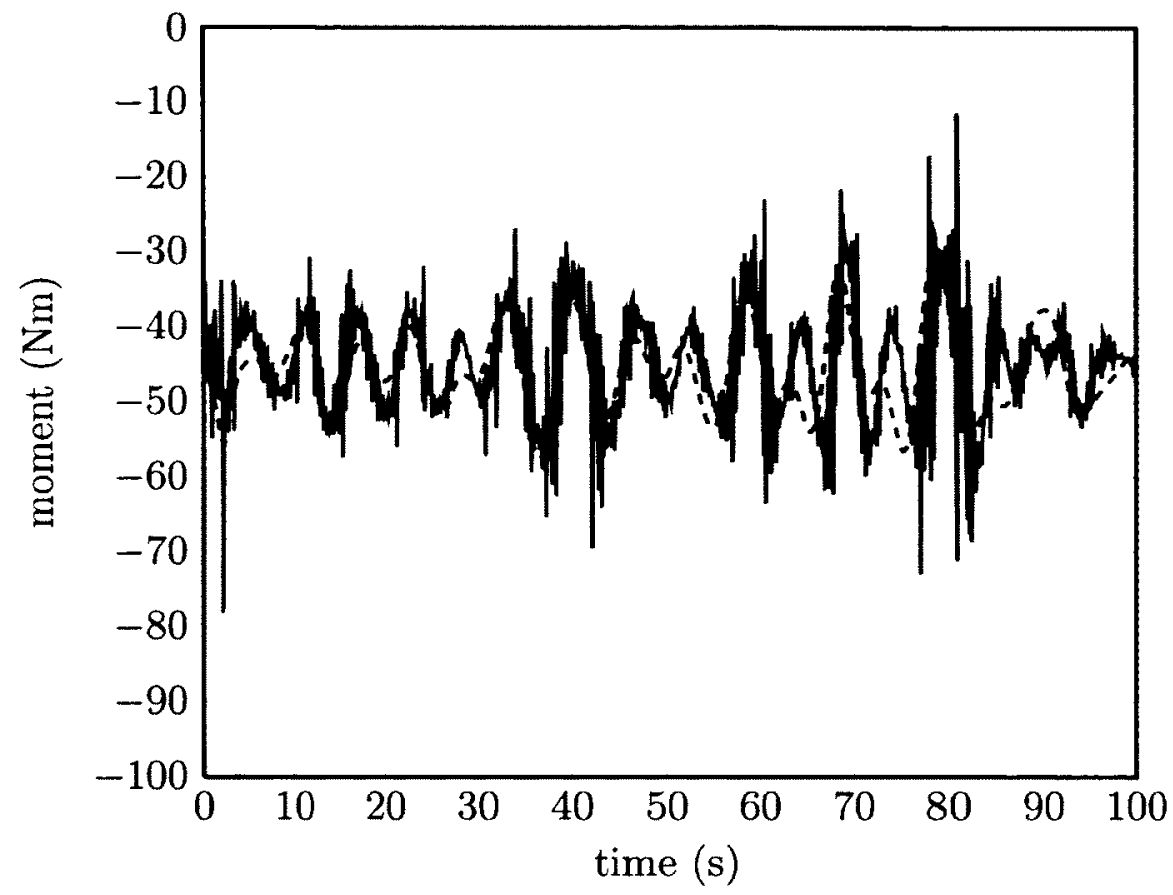

Figure 52: Lateral moment $\left(M_{y}\right)$ computed using GRM3D (solid) coupled to pendulum load and the load cell results from the experimentation (dashed).

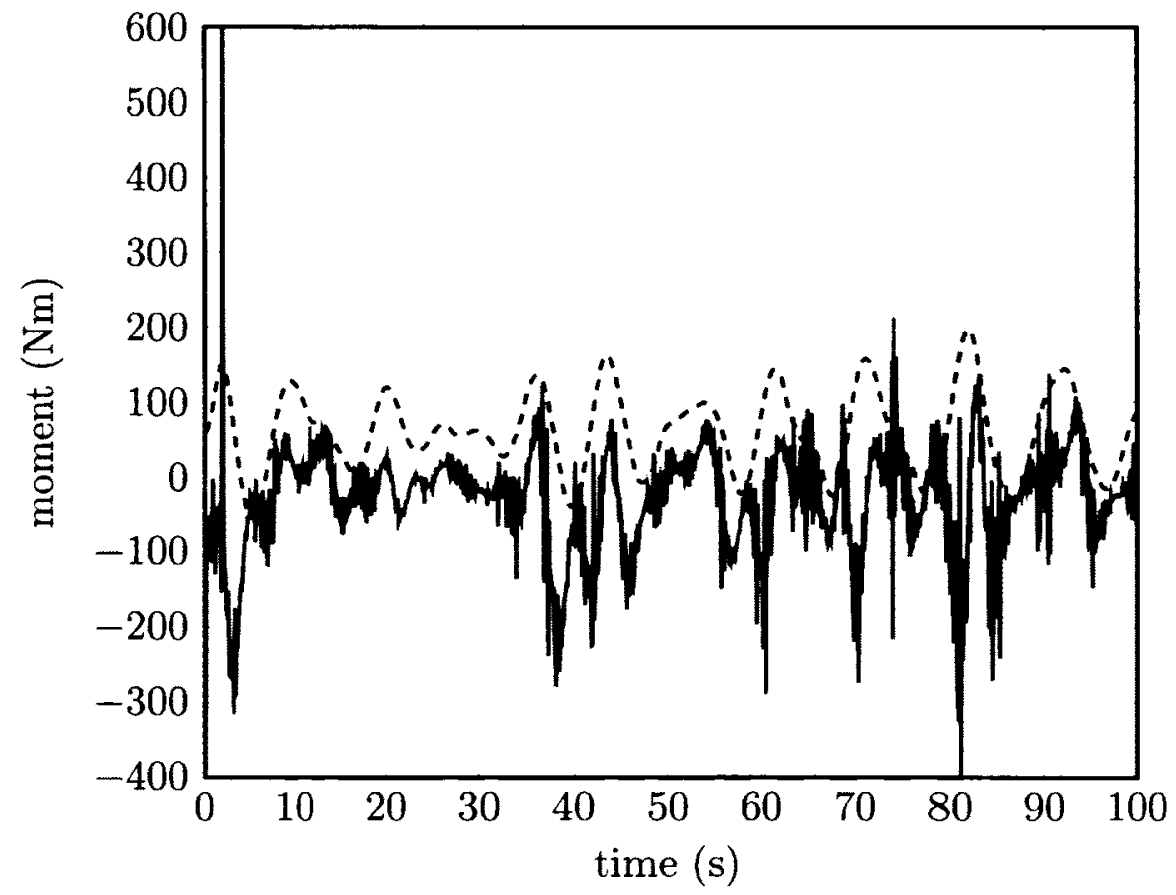

Figure 53: Vertical moment $\left(M_{z}\right)$ computed using GRM3D (solid) couple to pendulum load and the load cell results from the experimentation (dashed). 


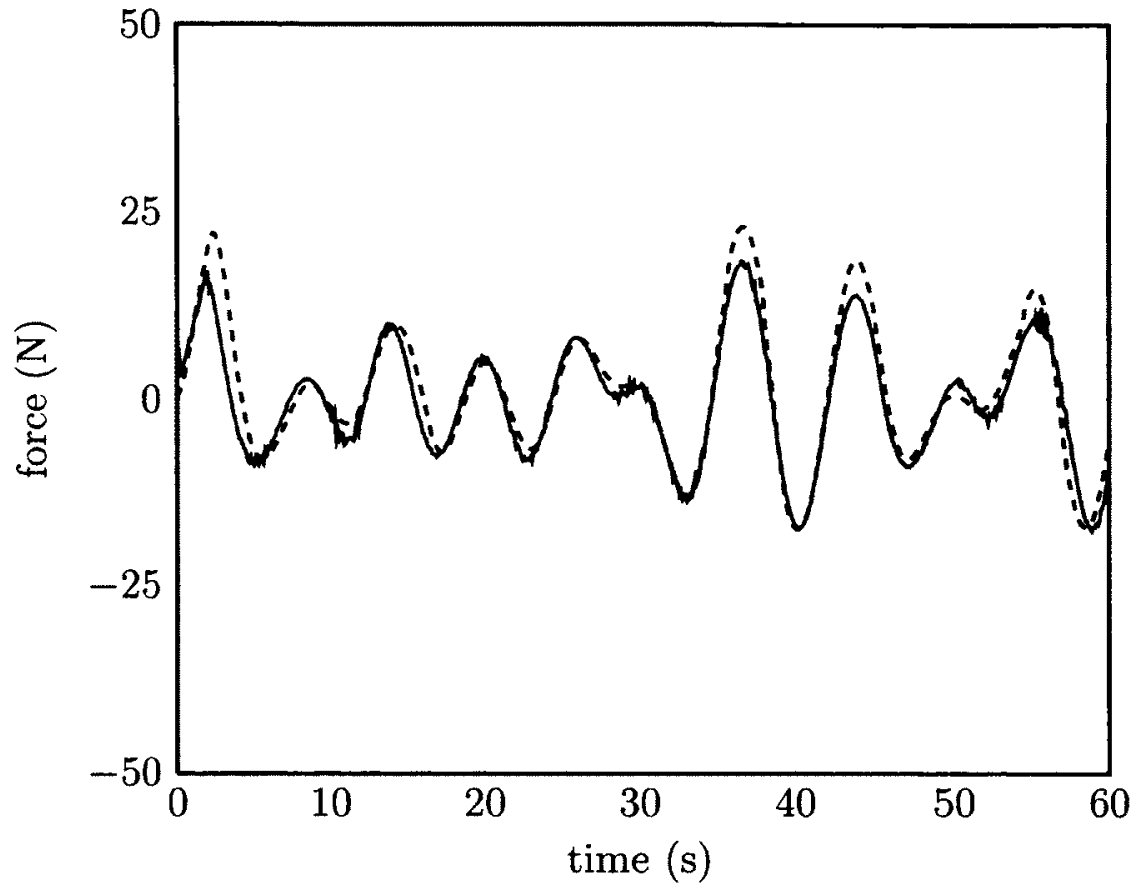

Figure 54: Longitudinal force $\left(F_{x}\right)$ computed using coupled GRM3D (solid) coupled to cart load and the load cell results from the experimentation (dashed).

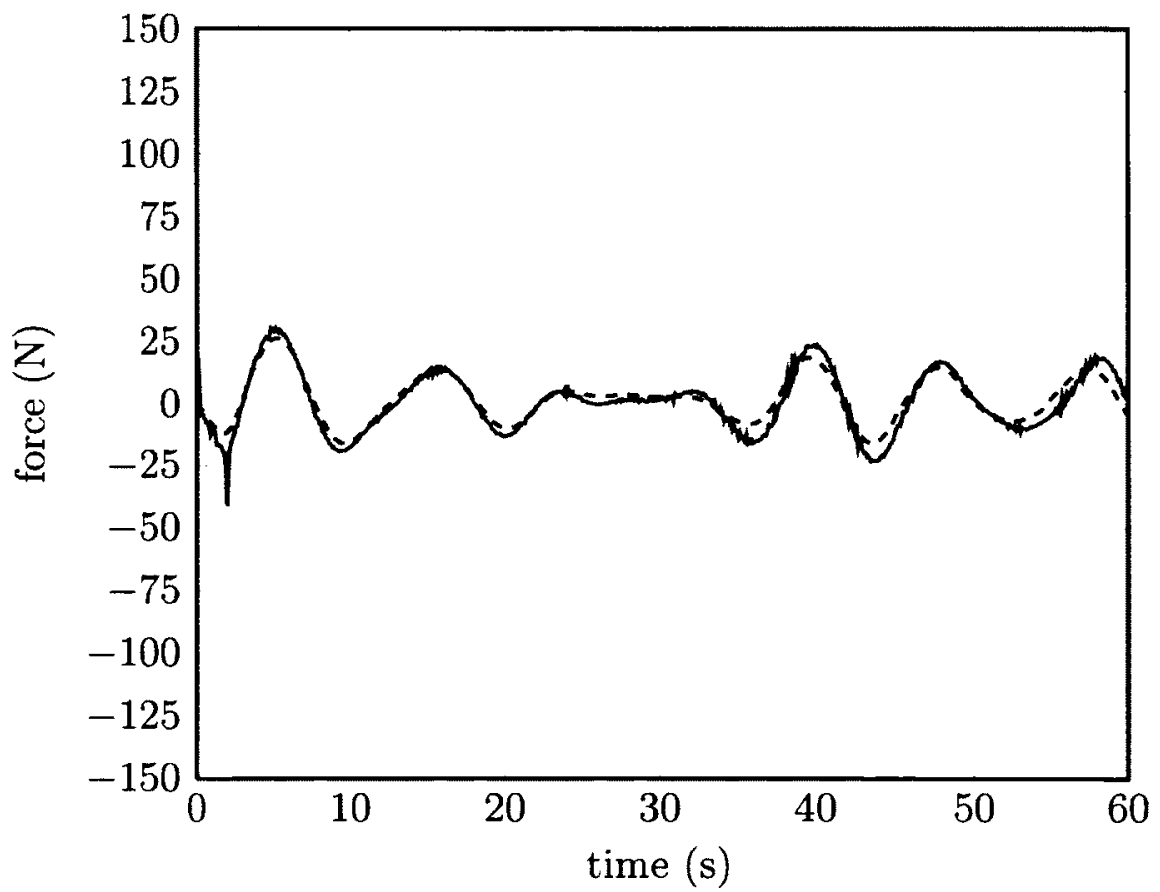

Figure 55: Lateral force $\left(F_{y}\right)$ computed using coupled GRM3D (solid) coupled to cart load and the load cell results from the experimentation (dashed). 


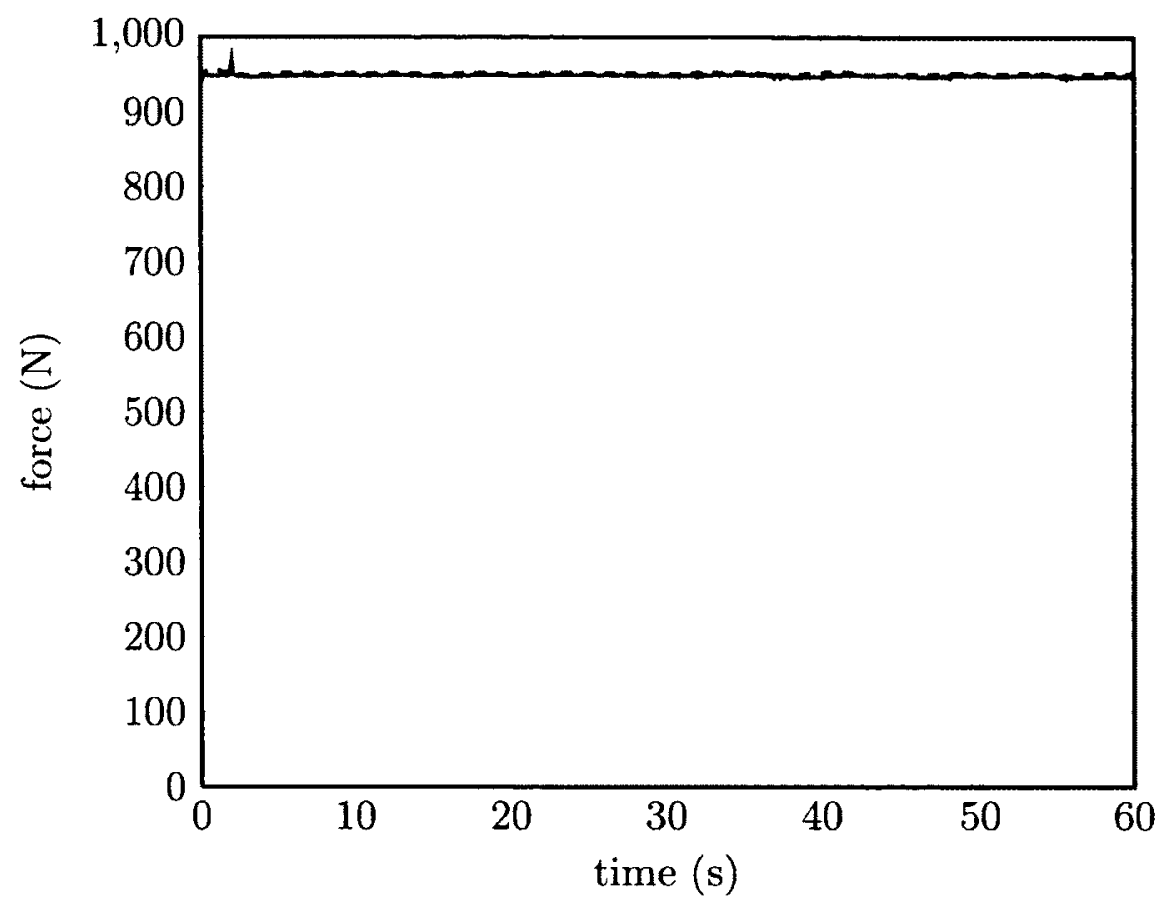

Figure 56: Vertical force $\left(F_{z}\right)$ computed using coupled GRM3D (solid) coupled to cart load and the load cell results from the experimentation (dashed).

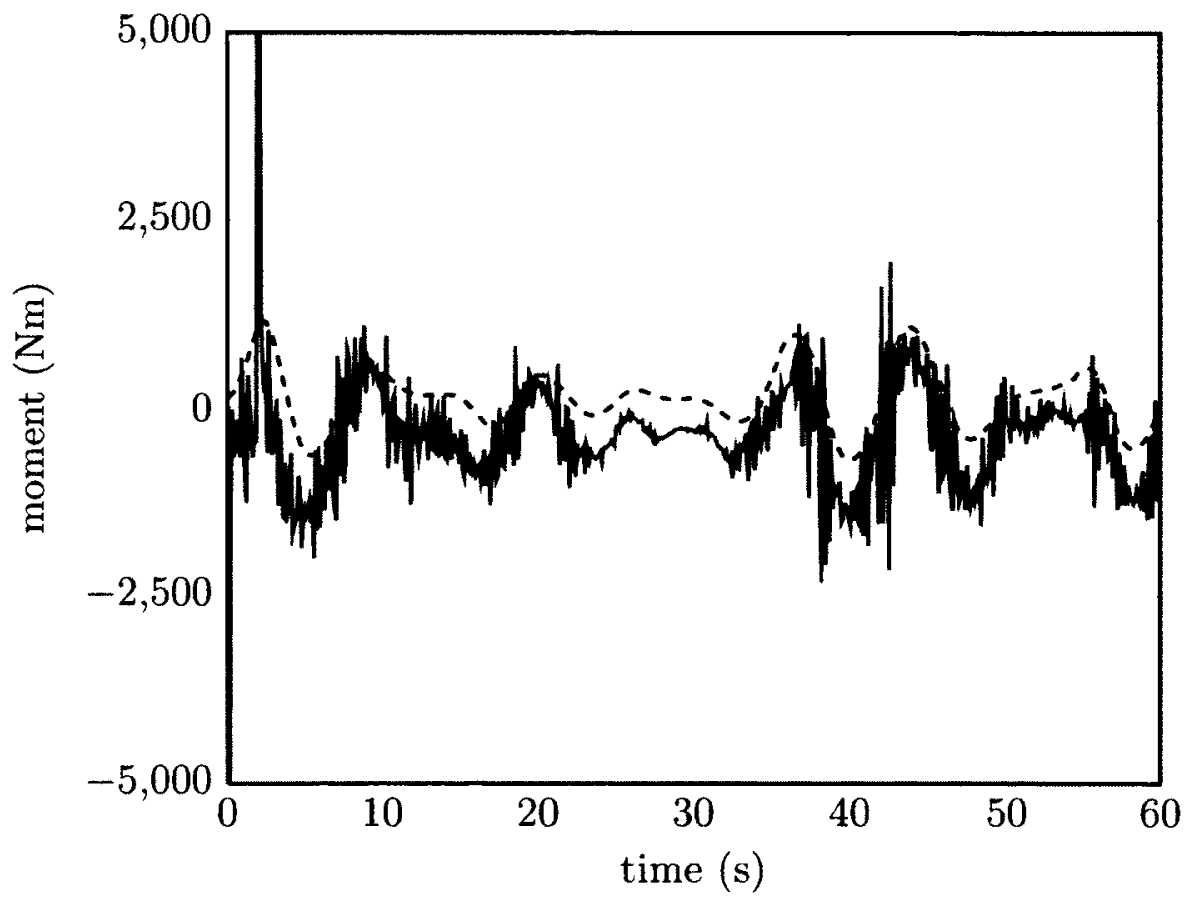

Figure 57: Longitudinal moment $\left(M_{x}\right)$ computed using coupled GRM3D (solid) coupled to cart load and the load cell results from the experimentation (dashed). 


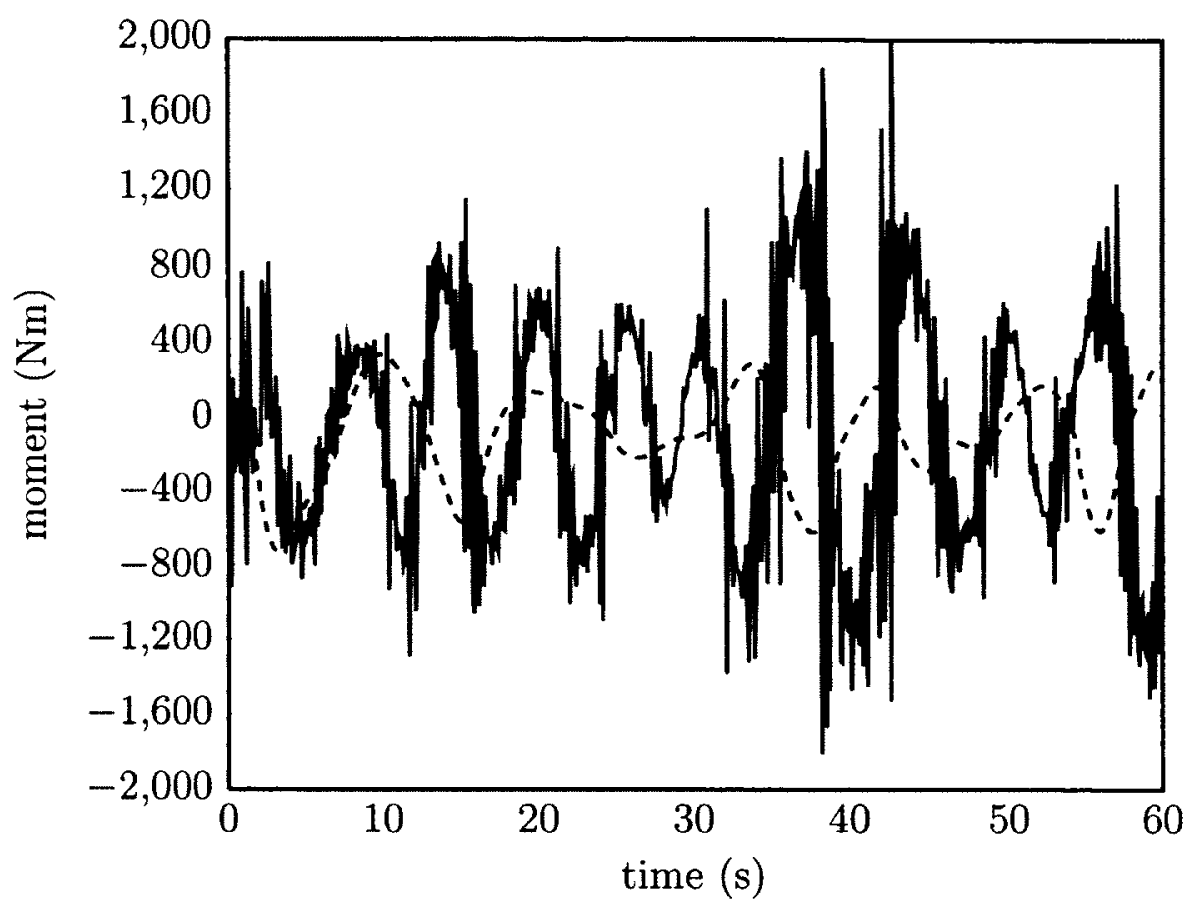

Figure 58: Lateral moment $\left(M_{y}\right)$ computed using coupled GRM3D (solid) coupled to cart load and the load cell results from the experimentation (dashed).

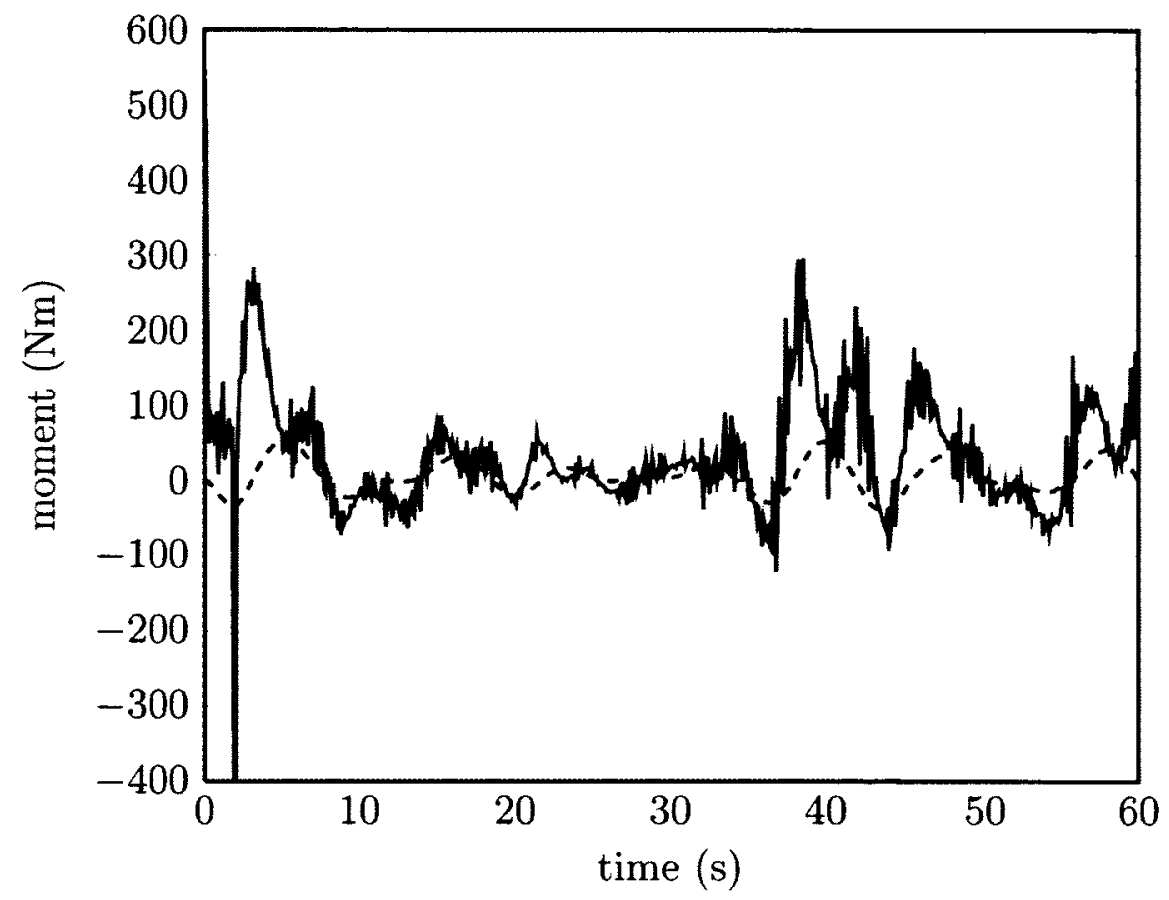

Figure 59: Vertical moment $\left(M_{z}\right)$ computed using coupled GRM3D (solid) coupled to cart load and the load cell results from the experimentation (dashed). 


\section{Chapter 4}

\section{Analysis}

\subsection{Definition and Concept}

There are four theoretically-possible modes of MII onset associated with the Graham stability model: sliding, tipping in the frontal plane, tipping in the sagittal plane, and yawing. Four parameters are used to identify these MII events. These parameters are defined in a general form such that they quantify the severity of the respective sea conditions without presupposing particular threshold values. The concept of an MII was first presented by Baitis and Applebee [33], and then subsequently generalized by Langlois [44] to the format used in this section.

In the case of sliding, the parameter called the sliding index $P_{\text {slide }}$ is given by

$$
P_{\text {slide }}=\frac{\sqrt{F_{x}^{2}+F_{y}^{2}}}{\left|F_{z}\right|}
$$

Sliding is expected when the value of $P_{\text {slide }}$ exceeds the prevailing coefficient of friction $\mu$ at the interface between the dynamic stability model and the deck.

In the case of tipping in the frontal plane, the relevant parameter called the frontal 
plane tipping index $P_{\text {frontal tip }}$ is given by

$$
P_{\text {frontal tip }}=\frac{\left|M_{x}\right|}{\left|F_{z}\right|}
$$

Tipping is expected when the value of $P_{\text {frontal tip }}$ exceeds a threshold corresponding to the effective stance width.

In the case of tipping in the sagittal plane, the relevant parameter called the sagittal plane tipping index $P_{\text {sagittal tip }}$ is given by

$$
P_{\text {sagittal tip }}=\frac{\left|M_{y}\right|}{\left|F_{z}\right|}
$$

Tipping is expected when the value of $P_{\text {sagittal tip }}$ exceeds a threshold corresponding the effective stance length.

In the case of yawing, the relevant parameter, called the yawing index $P_{\text {yaw }}$ is given by

$$
P_{y a w}=\frac{\left|M_{z}\right|}{\left|F_{z}\right|}
$$

Yawing is expected when the value of $P_{y a w}$ exceeds a threshold corresponding to the effective stance diameter. In summary, the reaction forces and moments determined from the stability models, are used to calculate these MII indices. They provide the means for estimating likely onsets of MII events for rectangular footprints.

\section{Modified Indices for a Generic Footprint}

The Graham block model's only means of resisting any applied moment about the $x$ and $y$ directions is through the location of the normal force. The same is true of the inverted pendulum as the current controller does not exploit inertial control strategies. If the normal force needs to be applied outside the bounds of the footprint, the model is said to tip over. The stance footprint available to generate a 
sufficient restoring moment was defined arbitrarily in order to generalize the tipping index. The required coordinates of the normal force is calculated based on this generalized footprint.

Assuming the postural model is no longer attached to the deck at the single point below the centre of mass, the normal force intersects the deck at a location, defined by $x$ and $y$ coordinates, appropriate for countering the tipping moments about the $x$ and $y$ directions. The corresponding distances required to counter the $x$ and $y$ moments are

$$
\begin{aligned}
& r_{y}=\frac{M_{x}}{F_{z}} \\
& r_{x}=\frac{M_{y}}{F_{z}}
\end{aligned}
$$

where $r_{x}$ is the distance in the $x$ direction of the normal force $F_{z}$ from the centre of mass and $r_{y}$ is the distance in the $y$ direction of the normal force from the centre of mass; all expressed in the model coordinate frame. The geometry is illustrated schematically in Figure 60. The calculated distances $r_{x}$ and $r_{y}$ define the location of the normal force on the ship deck in the model frame relative to the centre of mass. In order to determine a tipping incident, the next requirement is to determine whether the $x$ and $y$ location of the normal force is contained within the footprint.

It is assumed that the footprint of the Graham model is defined by $n$ points that, when connected in sequence, define a closed polygon. Each vertex of the polygon is required to be defined using Cartesian coordinates relative to the centre of mass. The location of the normal force would be either inside or outside the footprint polygon. An algorithm to find whether the normal force is within the polygon requires the angle between the point $\left(r_{x}, r_{y}\right)$ of the normal force and the $n$th and $n+1$ th vertex points, shown in Figure 61. Summing all the angles calculated 


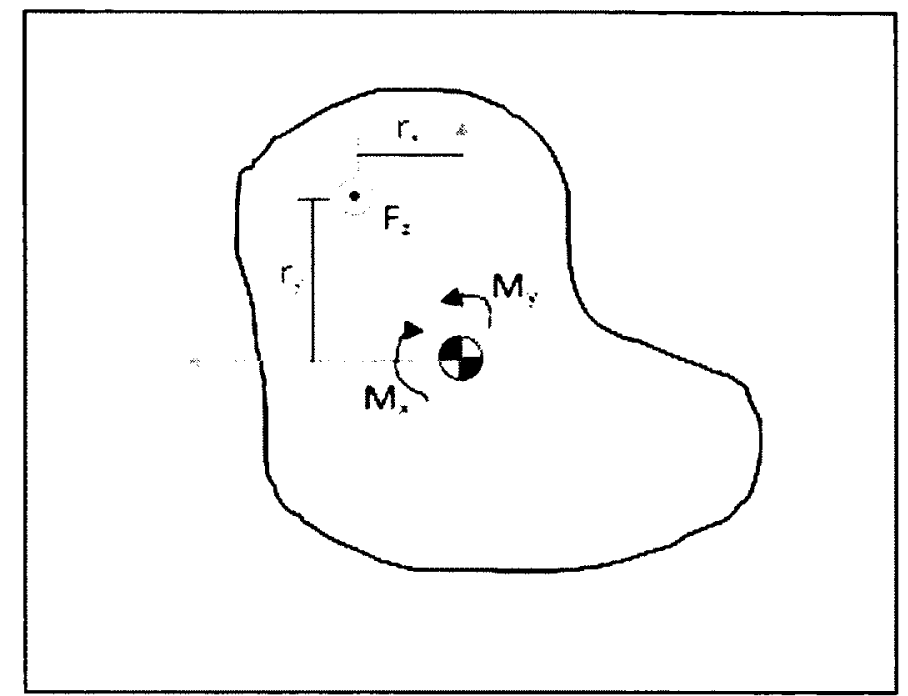

Figure 60: Normal force location to counteract the tipping moments on the block model with a generic footprint

between the vertices results in $360^{\circ}$ if the normal force is within the footprint, and $0^{\circ}$ if the normal force is outside. These values can be used as indicators for determining tipping events.

\subsection{Validation Experiments}

The footprint MII determination model presented is validated using a physical experiment. This section presents the design of the physical experimental apparatus for the MII experiments as well as the validation testing. The results from the experiments are summarized and are validated with data obtained on CFAV Quest.

\subsubsection{Physical Apparatus Design}

The experimental apparatus is based on the Graham model of a block with humanoid mass and inertial properties that can be seen in Figure 62. The apparatus is cut out of three-quarter inch SPF plywood in the shape of a stylized humanoid 


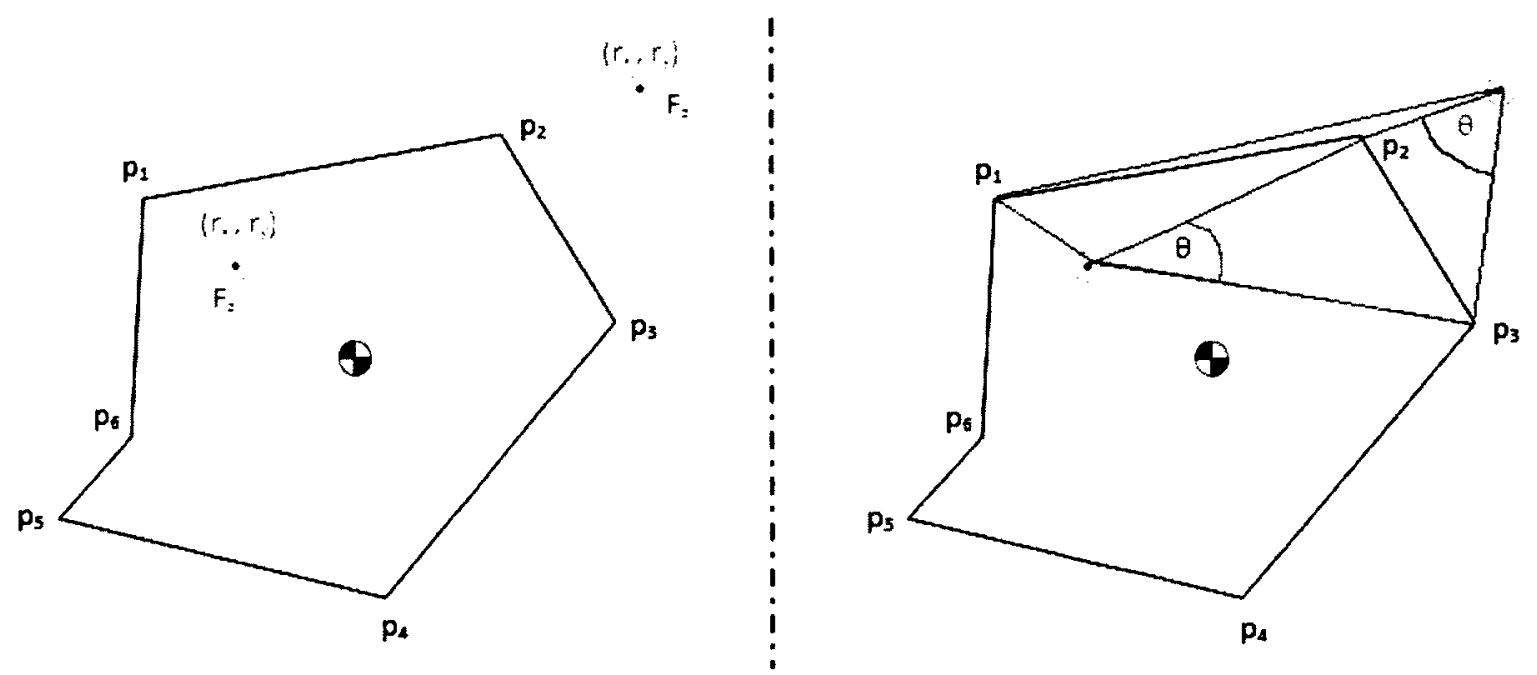

Figure 61: Determining the location of the normal force by the angle between the vertex points on the polygon footprint.

similar to that originally illustrated by Graham [34]. Three layers of plywood were laminated in order to achieve the desired thickness of the humanoid section. The scaled drawing of the assembled apparatus is provided in Figure 63. The contact surface of the model is attachable to the humanoid section using wood screws. Two separate contact footprints were constructed for the stance of the model, one with the feet side by side and one with the feet staggered with one foot ahead of the other. A scaled drawing has been provided in Figure 64. The side by side stance should correspond to the results from the Graham model as the footprint has rectangular edges. The staggered footprint was chosen because it is a common alternative stance to the side by side stance. A photo of the physical apparatus is provided in Figure 65.

To measure the time and direction of the MII occurrences for the physical apparatus, on/off contact switches are attached along the outside perimeter of the footprint. Each of the switches is connected to a microcontroller manufactured by Arduino. This microcontroller is programmed to identify which one of the switches are depressed at each time interval. The switches have been attached to the apparatus such that there is a known angle from the centre of mass to the individual switch locations. From the 


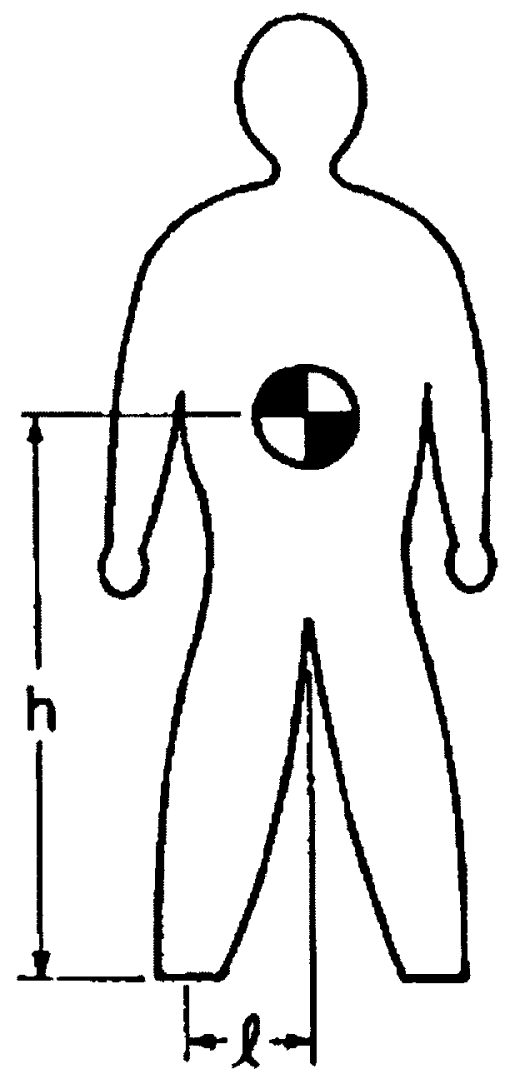

Figure 62: Stability model presented by Graham for MII detection [35].

switch locations the direction of tipping is known. It must be noted that this model is instrumented to only detect tipping MIIs of the stability model. Any sliding or yawing MII events must be observed manually. This is not particularly limiting as tipping MII events usually occur first with realistic interface parameter values.

\subsubsection{Detection with Stance Geometry Validation Ex- periments}

The goal of these experiments was to validate that the MII events predicted by the stance model agreed in number and occurrence time with those measured during 

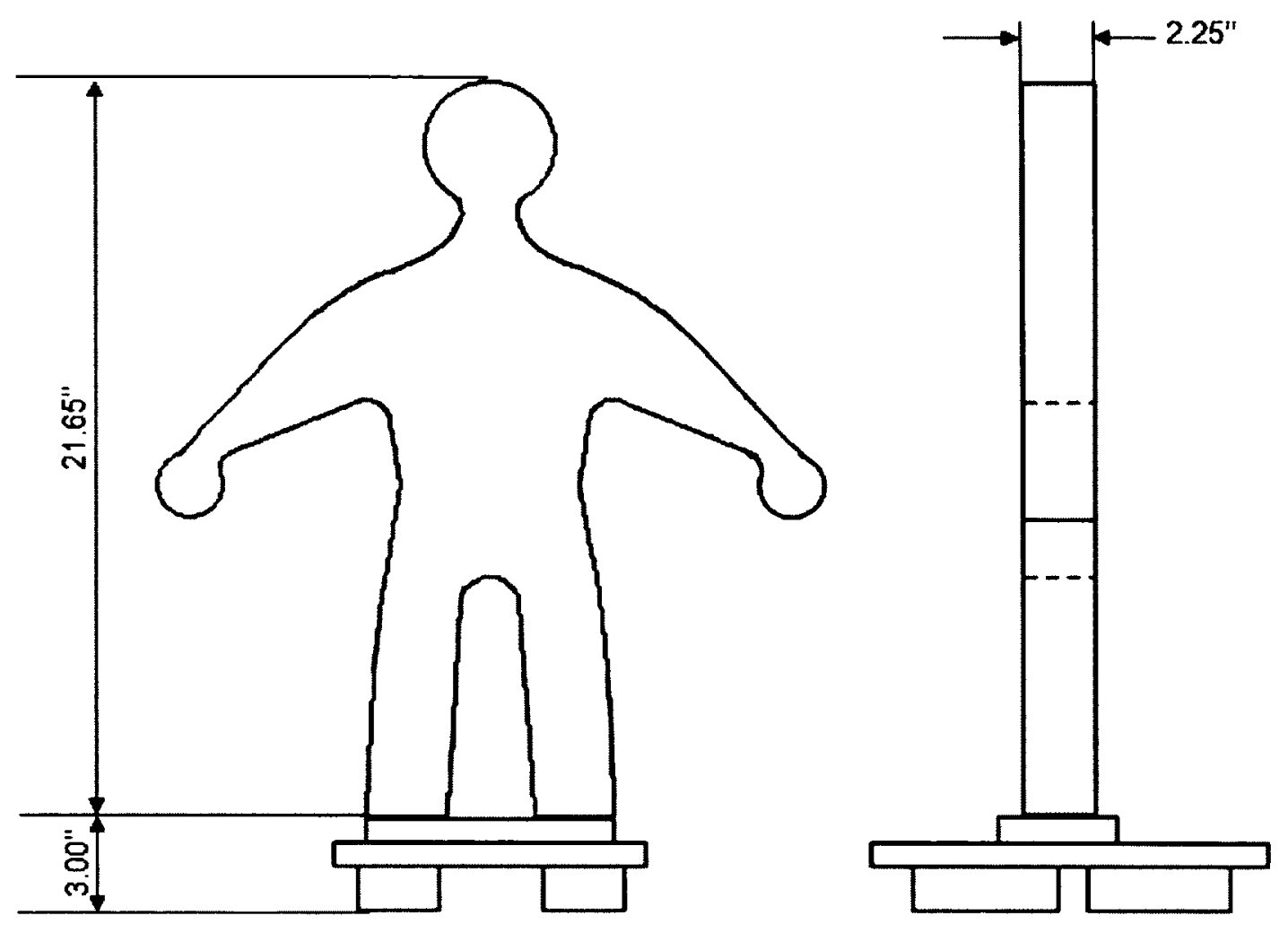

Figure 63: Scaled drawing of the MII man assembled apparatus with staggered footprint attached.

the experiment. The validation experiments are designed in order to fulfill this requirement. The experiments consisted of placing the experimental apparatus on the six-degree-of-freedom motion platform and then to subject the apparatus to simulated ship motions. The ship motions used for the validation experiments corresponded to seas characterized by sea state five with long-crested waves of 10 metre significant wave height. The simulated ship is travelling at a heading of forty-five degrees relative to the wave direction. The resulting ship motions were predominantly in the roll direction. To keep the motions within the platform limits, translational motions were excluded. Each of the two stance geometries were run with the apparatus facing at 0 , 45 , and 90 degrees relative to the simulated ship's longitudinal axis. This experiment 
can only validate the MII stance model for the Graham model. It is not possible to attach the MII experimental apparatus with the postural stability load apparatus due to differences in their scales.

The data collected from the Arduino microcontroller was written to an output file where a depressed switch was indicated by a 1 versus a 0 if not depressed. The file also contains which of the switches were being depressed at a given time. The switches were labeled on the physical model to ensure the position of each switch was known. This file was interpreted manually in order to count the MIIs and the time when each MII occurred. An MII was said to occur if the depression of the switches changed. It was also necessary to investigate the rate of change of the switches because the model would occasionally sway back and forth prior to stabilizing. Based on the definition of an MII, each swaying motion in such a case should not be considered as a distinct MII because they are all considered a part of regaining balance following the initial MII. The MII event is used to determine the onset of imbalance, and the count is not allowed to proceed until balance is restored. In reality, this means that if a person has lost balance and remains unbalanced for a long time it only counts as one MII event until full balance is restored. This is interpreted in the data by only investigating the time at which the switch depression is changed and held in this state for over two seconds. This was assumed sufficient time to say that full balance was restored.

The simulation was run using the same ship motions as in the physical experiments. The physical parameters of the MII experimental apparatus are also known and used for the simulation. The results from the simulation are provided in a similar format to the results from the switches on the physical apparatus. The $x$ and $y$ coordinates of the normal force are used to determine if the force is being applied within the footprint. For each time step the point is checked with the footprint dimensions and is said to be within the footprint with a value of 1 and outside of the footprint with a value of 0 . In order to determine the occurrence of an MII event this data 
was visually checked. An MII was said to occur if there was a change in the normal force position from inside the footprint to outside the footprint. Times also occurred in the collected data at which point the normal force would change from being inside to being outside the footprint rapidly on the edge of the stance. This was counted as one MII event because the balance was not truly restored. Balance was said to be restored if there was no change in stance value for 2 seconds.

\subsubsection{Comparison of Results from the MII Stance Experi- ments}

This section presents the comparison of the results from the physical experiments with these from the simulation. Results will be presented for both of the stances considered: the side by side (also named straight), and the staggered footprints. A complete set of straight results will be presented first.

The motion was run for 100 seconds to collect the data. The ship motions were used to run the simulation and the number of MIIs were counted from the output based on whether the normal force was within the stance geometry or not. Visualization of the movement of the normal force within the footprint can be seen in Figure 66 with the cross points being within the footprint and circle points being outside. The MIIs from the physical experiment and the simulation where the model was at zero degrees were counted and the times of occurrence of the MIIs can be seen in Table 12 .

The same procedure was performed for the staggered footprint. The results from the experimental apparatus and the simulation for the apparatus oriented at zero degrees can be seen in Table 13. As well, a graph for visualization of the $x$ and $y$ positions in terms of the footprint coordinates is provided in Figure 67. The graph of the normal force coordinates shows the location of the normal force required to maintain stability on the deck $x$ and $y$ plane. The solid line represents the perimeter 
Table 12: MII occurrence times for the straight stance at 0 degrees offset obtained from the experimental apparatus and simulation

\begin{tabular}{ccc}
\hline MII Number & Occurrence Time - Experimental & Occurrence Time - Simulation \\
\hline 1 & $3.1 \mathrm{sec}$ & $2.62 \mathrm{sec}$ \\
2 & $5.96 \mathrm{sec}$ & - \\
3 & $72.65 \mathrm{sec}$ & $70.31 \mathrm{sec}$ \\
4 & $82.45 \mathrm{sec}$ & $79.75 \mathrm{sec}$ \\
\hline
\end{tabular}

of the footprint. Since the definition of an MII is defined when the normal force crosses to outside the footprint, this can be seen in the graph by when the points change from within to outside the footprint perimeter drawn in the graph.

\subsubsection{Validation Against Footprint Model Experimentation on CFAV Quest}

CFAV Quest is a research vessel operated by DRDC Atlantic for sea trial experiments. From November 20th through 28th Quest departed from Halifax, Nova Scotia, for a heavy weather sea trial in the North Atlantic Ocean. A postural stability experiment from Carleton University's Applied Dynamics Lab was one of two experiments conducted through the trial. Among other related experiments comprising the Carleton experiments, the MII man experimental apparatus was brought on board in order to collect data for the footprint model. It was set up on the deck in the lab space provided on the ship. The data collection was similar to the data collected in the Applied Dynamics Lab at Carleton. The ship motion was measured with a six-degree-of-freedom inertial sensor that recorded accelerations, angular rates, and angles of the deck. The day of the data collection, the waves were measured at an approximate height of $2.5 \mathrm{~m}$ (low sea state 5 ) which provided MII events but allowed 
Table 13: MII occurrence times for the staggered stance at 0 degrees offset obtained from the experimental apparatus and simulation

\begin{tabular}{ccc}
\hline MII Number & Occurrence Time - Experimental & Occurrence Time - Simulation \\
\hline 1 & $2.87 \mathrm{sec}$ & $1.64 \mathrm{sec}$ \\
2 & $4.76 \mathrm{sec}$ & $6.26 \mathrm{sec}$ \\
3 & $9.66 \mathrm{sec}$ & $12.23 \mathrm{sec}$ \\
4 & $15.23 \mathrm{sec}$ & $17.13 \mathrm{sec}$ \\
5 & $20.80 \mathrm{sec}$ & - \\
6 & - & $30.12 \mathrm{sec}$ \\
7 & - & $36.20 \mathrm{sec}$ \\
8 & $39.49 \mathrm{sec}$ & $40.56 \mathrm{sec}$ \\
9 & $43.87 \mathrm{sec}$ & $44.87 \mathrm{sec}$ \\
10 & $48.64 \mathrm{sec}$ & $54.51 \mathrm{sec}$ \\
11 & $61.69 \mathrm{sec}$ & $58.53 \mathrm{sec}$ \\
12 & $66.36 \mathrm{sec}$ & $63.36 \mathrm{sec}$ \\
13 & $70.76 \mathrm{sec}$ & $68.21 \mathrm{sec}$ \\
14 & $76.01 \mathrm{sec}$ & $73.17 \mathrm{sec}$ \\
15 & $79.19 \mathrm{sec}$ & $78.45 \mathrm{sec}$ \\
16 & $80.95 \mathrm{sec}$ & - \\
\hline
\end{tabular}

the apparatus to maintain balance for most of the experimental time. The data was collected in 2 minute sets where the apparatus was oriented at 0,45 , and 90 degrees relative to the longitudinal axis of the ship. Both the straight and staggered footprints were used.

The number of MIIs were counted from the data obtained using the switches and were compared to the results from the simulation. The simulation was run using the ship motion collected from the inertial sensor and the physical parameters tabulated in the previous section. The occurrence of MIIs for the straight stance at 90 degrees 
Table 14: MII occurrence times for the straight stance at 90 degrees in beam seas obtained from the experimental apparatus on Quest experiments and simulation

\begin{tabular}{ccc}
\hline MII Number & Occurrence Time - Experimental & Occurrence Time - Simulation \\
\hline 1 & $4.41 \mathrm{sec}$ & $5.8 \mathrm{sec}$ \\
2 & $8.08 \mathrm{sec}$ & - \\
3 & $26.4 \mathrm{sec}$ & $28.12 \mathrm{sec}$ \\
4 & $31.9 \mathrm{sec}$ & $30.55 \mathrm{sec}$ \\
5 & $42.2 \mathrm{sec}$ & $40.44 \mathrm{sec}$ \\
6 & $55.9 \mathrm{sec}$ & $47.27 \mathrm{sec}$ \\
7 & $65.3 \mathrm{sec}$ & $61.03 \mathrm{sec}$ \\
8 & $79.9 \mathrm{sec}$ & $66.25 \mathrm{sec}$ \\
9 & $90.1 \mathrm{sec}$ & $85.83 \mathrm{sec}$ \\
\hline
\end{tabular}

relative to the longitudinal axis of the ship with beam seas can be seen in Table 14 . From this data, the 2 second delay can be seen from the simulation data as well. Table 15 provides the MII occurrences for the staggered stance at 0 degrees relative to the longitudinal axis of the ship with beam seas. The data from other model orientations and ship heading also produced similar results. These results show that the footprint model has been validated through experimentation on a ship in real world conditions at sea. 
Table 15: MII occurrence times for the staggered stance at 0 degrees in beam seas obtained from the experimental apparatus on Quest experiments and simulation

\begin{tabular}{ccc}
\hline MII Number & Occurrence Time - Experimental & Occurrence Time - Simulation \\
\hline 1 & $32.7 \mathrm{sec}$ & $31.41 \mathrm{sec}$ \\
2 & $42.76 \mathrm{sec}$ & $42.3 \mathrm{sec}$ \\
3 & - & $52.75 \mathrm{sec}$ \\
4 & $68.6 \mathrm{sec}$ & $64.72 \mathrm{sec}$ \\
5 & $78.1 \mathrm{sec}$ & $72.68 \mathrm{sec}$ \\
6 & $87.7 \mathrm{sec}$ & $82.04 \mathrm{sec}$ \\
\hline
\end{tabular}




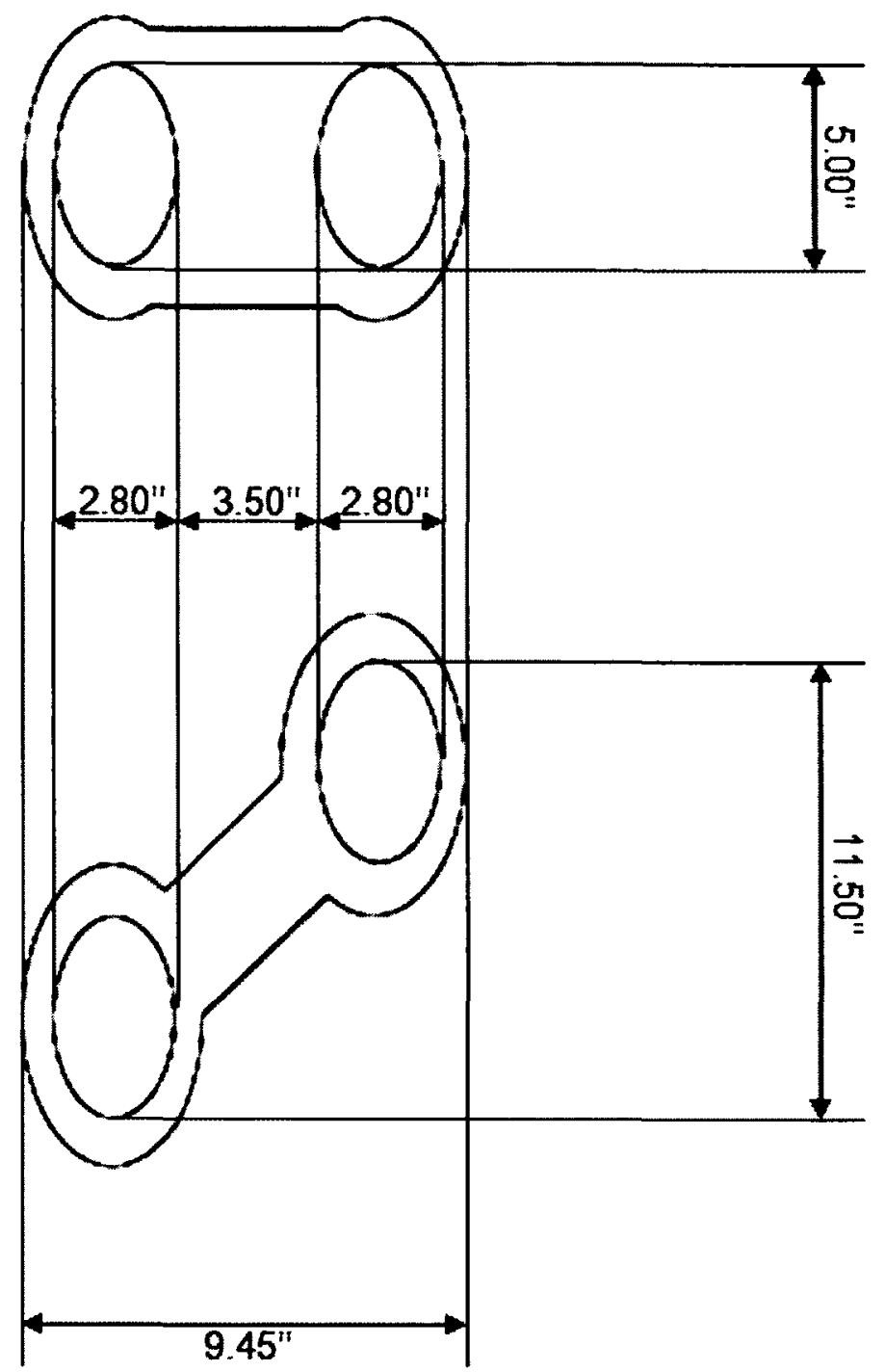

Figure 64: Scaled drawing of the straight and staggered footprint configurations. 


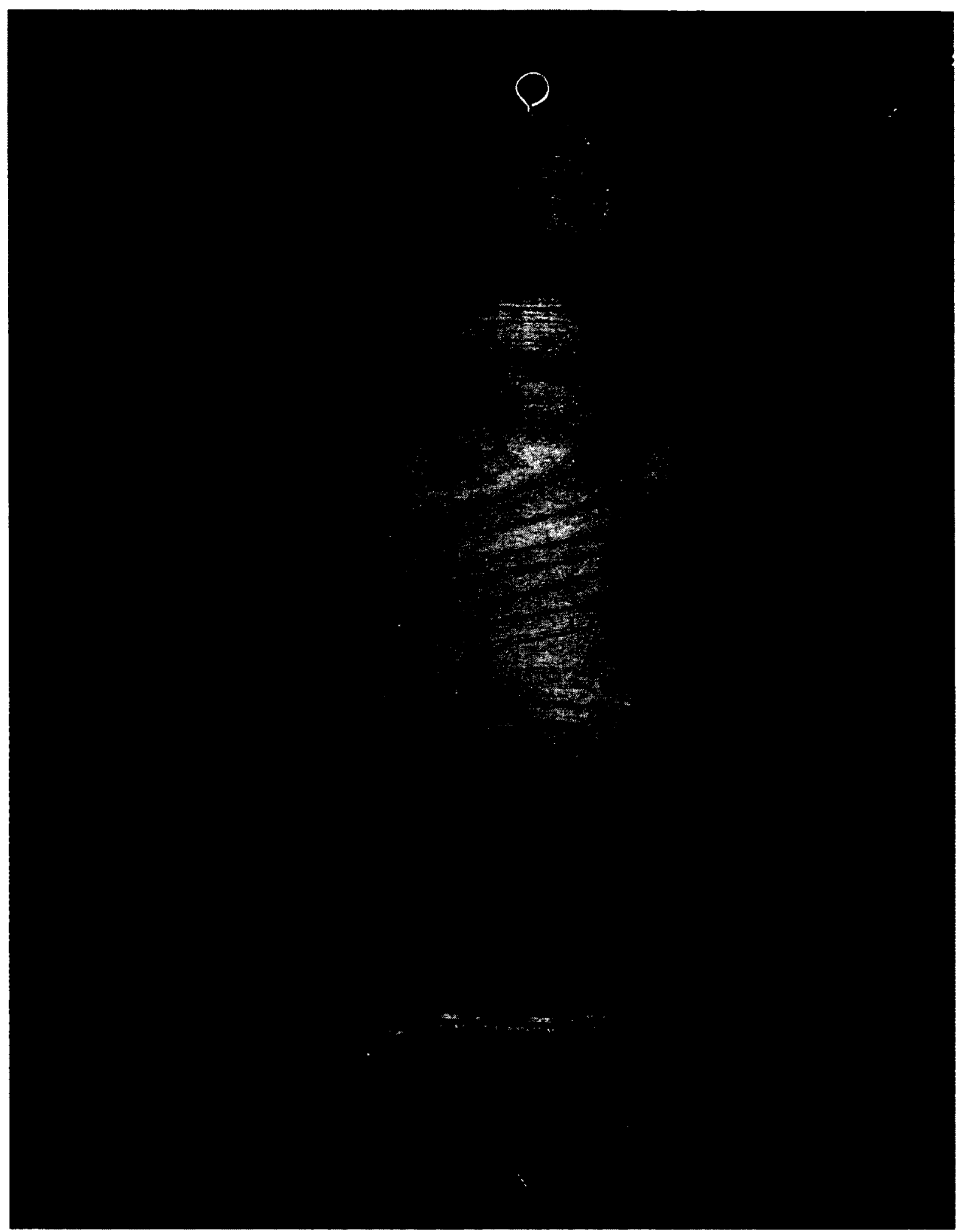

Figure 65: Physical apparatus with staggered footprint attached for the MII stance model validation. 


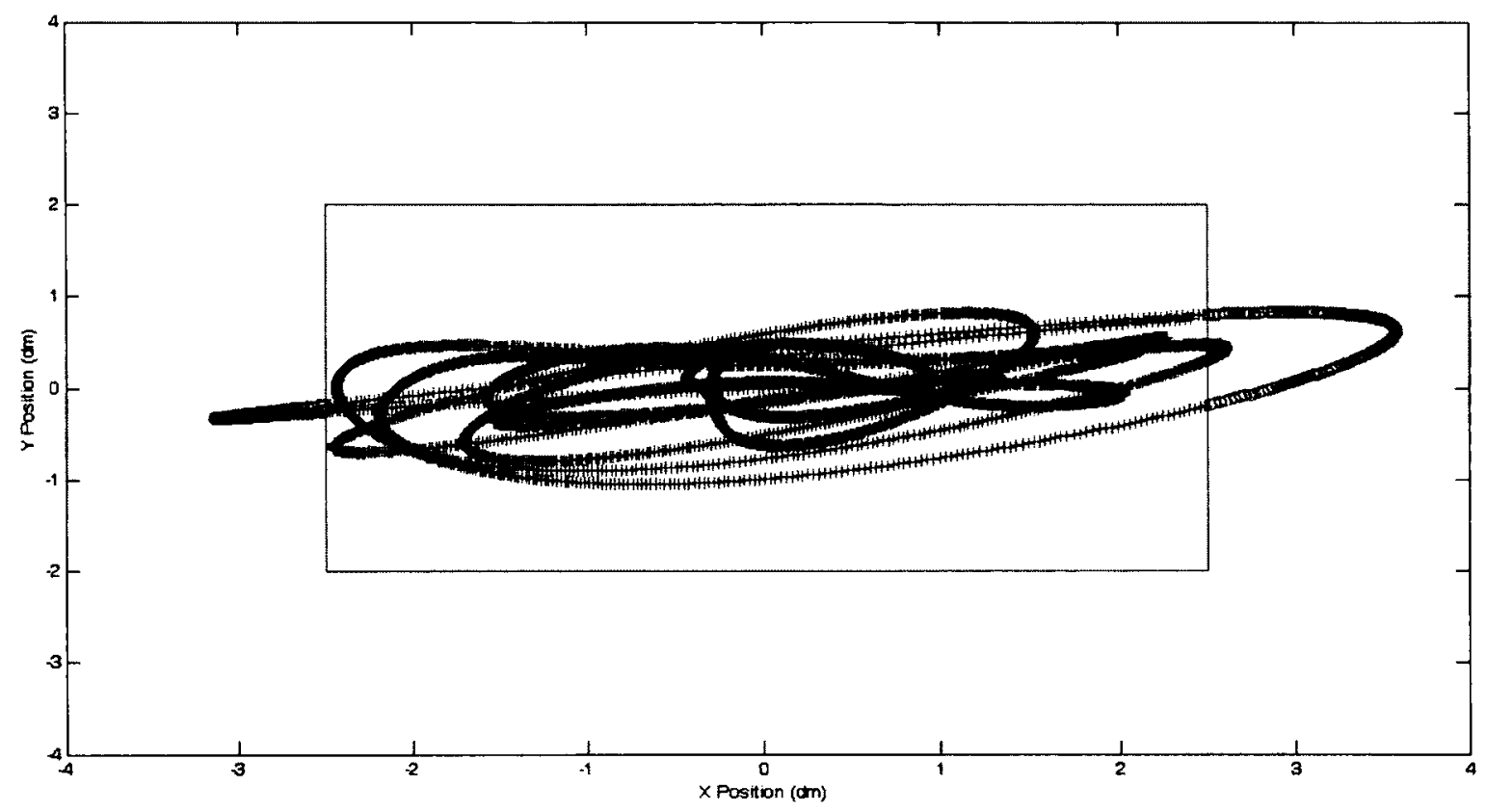

Figure 66: Coordinates of the normal force plotted about the straight footprint from simulation.

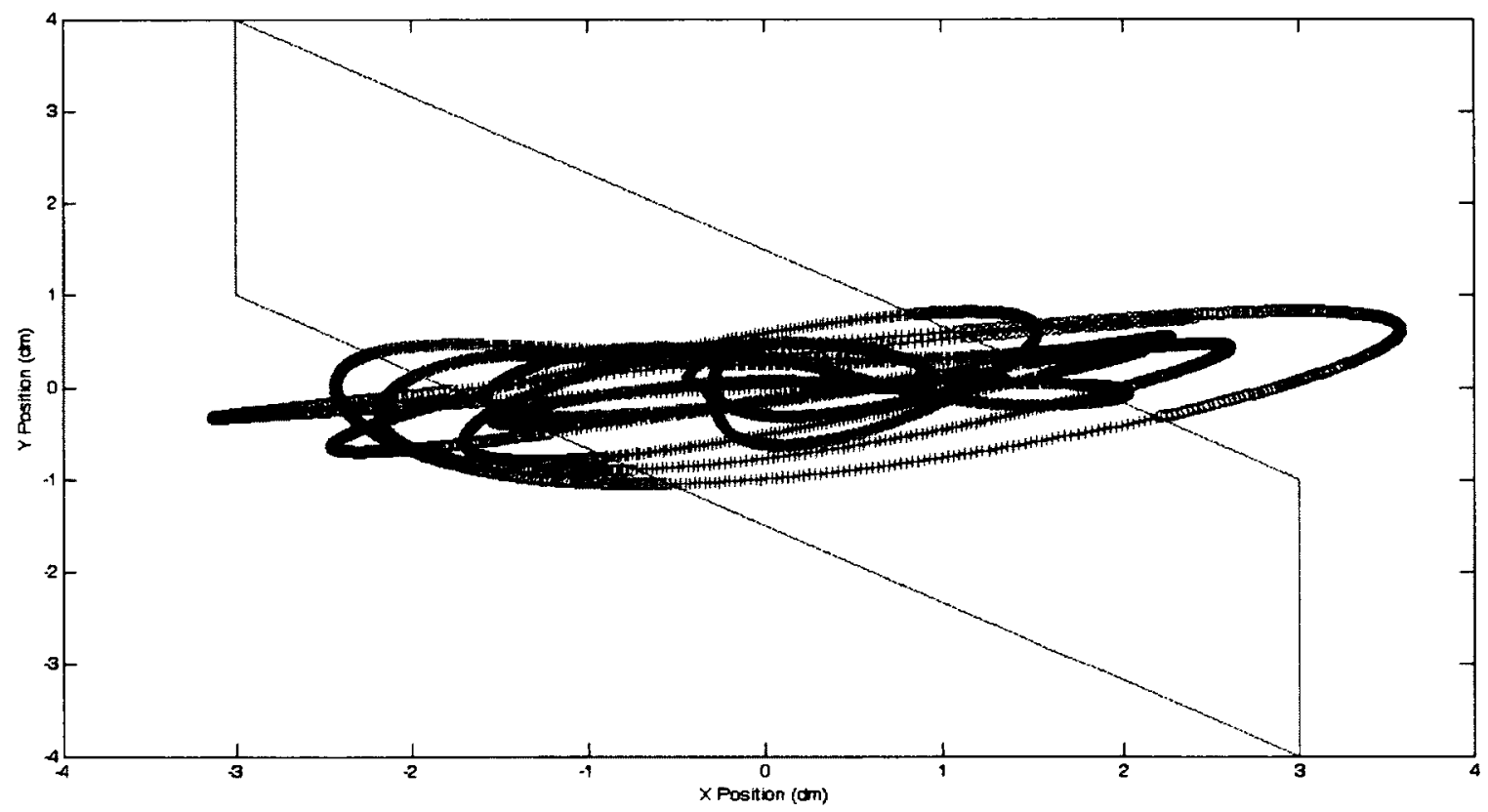

Figure 67: Coordinates of the normal force plotted about the staggered footprint from simulation. 


\subsection{Effect of Stance Geometry}

The previous section outlined the experimentation used to validate the stance model for the occurrences of MIIs. This section provides an investigation of the effect of stance geometry for a human-sized model. Three different sea states were investigated, as well as staggered and straight stance geometries.

Parameters that change the stance geometry for a person are the size of their feet and the placement and orientation of their feet relative to each other. For this investigation it was assumed that two people of the same mass and physical characteristics were standing with a straight foot stance. One of the people was perscribed to have a larger foot length than the other person. The effect of foot size was investigated by changing the length of the rectangular footprint. A 100 second simulation was run corresponding to sea state 6 conditions with a heading of 90 degrees relative to the principal wave direction. This simulation case was chosen because a higher rate of MIIs occur at this sea state. Two different stances were used for this investigation. The two stances had the feet parallel to each other $50 \mathrm{~cm}$ apart. One of the stances was assumed to be a smaller foot with a length of 6 US which is about $25 \mathrm{~cm}$. The other stance was assumed to be a larger foot with a shoe length of 10 US which is about $30 \mathrm{~cm}$. For the first test, the two models are set to be facing in the $x$ direction. The results from the test for the smaller footprint can be seen in Figure 68 and the larger footprint in Figure 69. In those two simulations the number of MIIs did not change between the two foot sizes. It can however be seen that there is a limit where the bigger foot size allows for a longer time being steady, because the normal force is within the footprint for longer. Another test was performed where the two models are perscribed to be facing the $y$ direction. The results for the smaller footprint can be seen in Figure 70 and for the larger footprint in Figure 71. Comparing these two cases identifies that one more MII is predicted for the smaller foot than the larger 


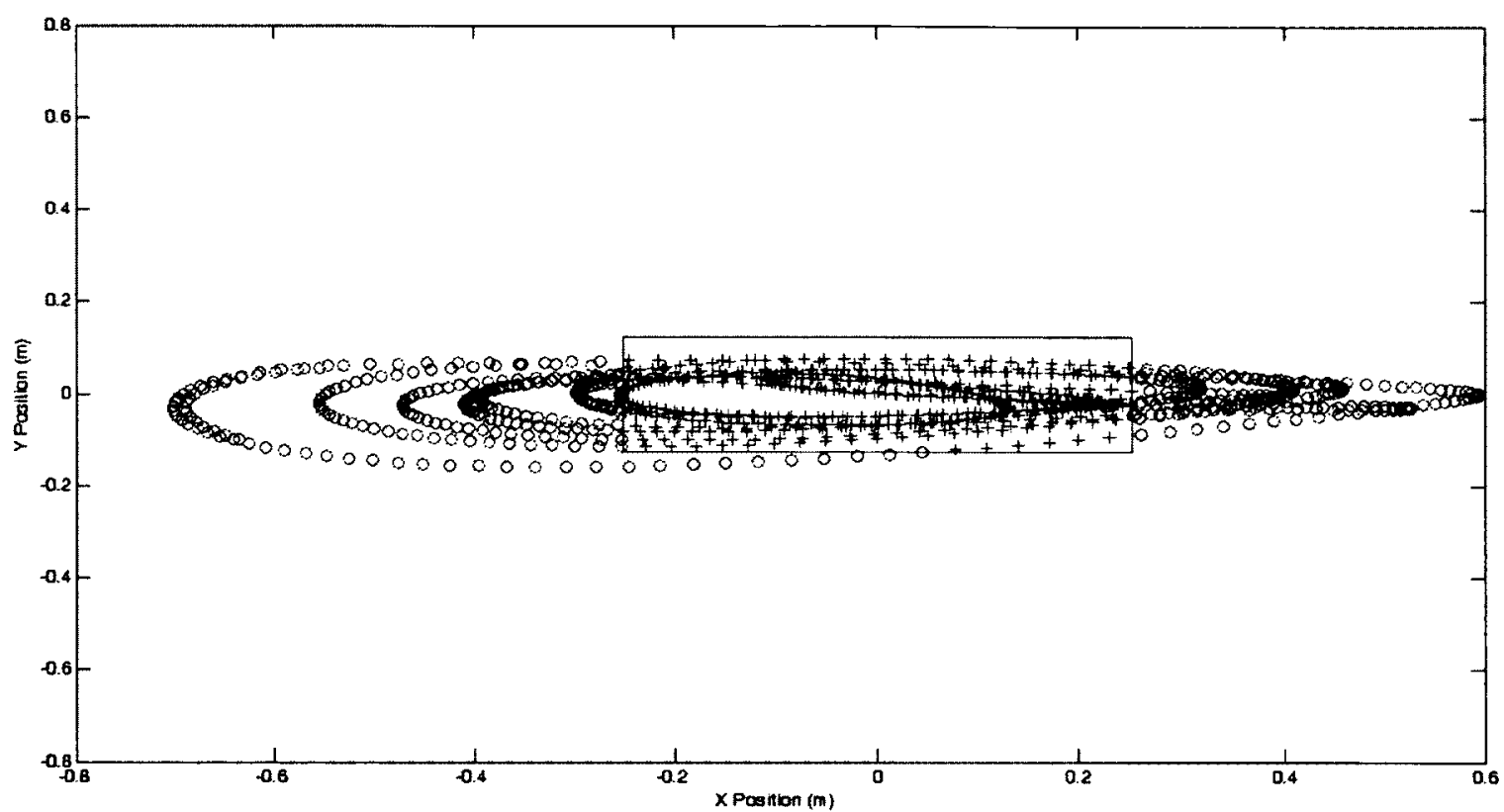

Figure 68: Coordinates of the normal force for $25 \mathrm{~cm}$ foot length footprint facing the $x$ direction.

foot. The increase in the number of MIIs is not large but it amounts to 36 more MIIs for the smaller foot every hour versus the larger foot. It should be noted that the assumption of the two people in these cases having the same physical parameters is not probable. These cases were designed in order to see the effects of each parameter individually in order to determine relationships from each parameter on the MII rate.

Another parameter that can be modified by someone on a ship is the width of their stance. In the previous test it was assumed that the person had their feet 50 $\mathrm{cm}$ apart from one outer edge to the other. The next investigation is to see how the number of MIIs change if they were standing with feet $75 \mathrm{~cm}$ apart and $100 \mathrm{~cm}$ apart. These cases use the same ship motion as the previous numerical experiment. The length of the foot is assumed to be $30 \mathrm{~cm}$ for both of these cases. It can be seen from Figures 72 and 73 that an increase in the stance width from $75 \mathrm{~cm}$ to $100 \mathrm{~cm}$ will decrease the number of MIIs by 5 every 100 seconds. 


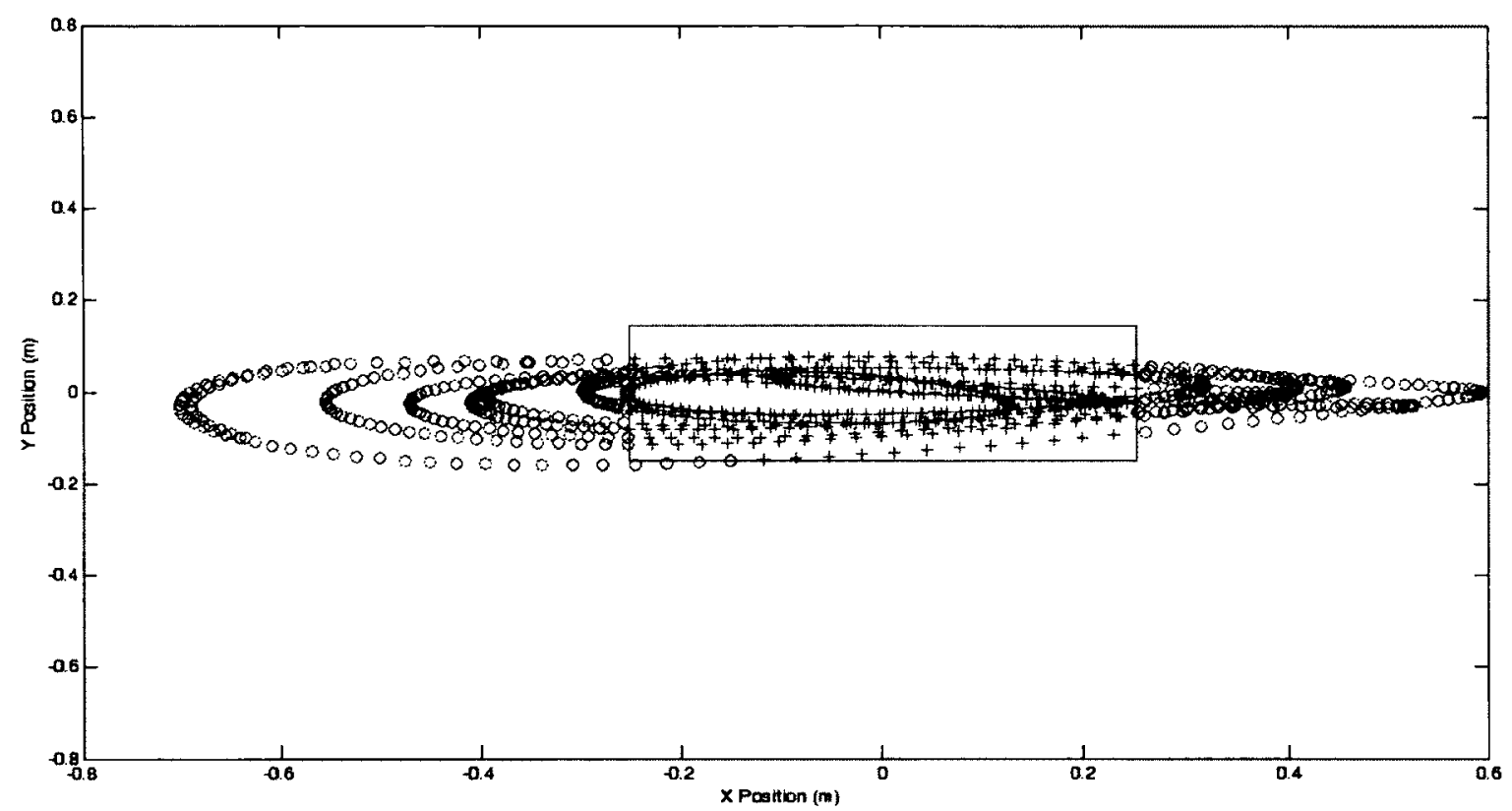

Figure 69: Coordinates of the normal force for $30 \mathrm{~cm}$ foot length footprint facing the $x$ direction.

Ships can travel at any heading relative to the principal wave direction subject to operational constraints. Therefore, the effect of the ship heading on the optimum stance angle to reduce the number of MIIs was also investigated. The angle of the ship heading is defined as the angle between the $x$ direction of the ship, along the longitudinal axis of the ship directed from stern to bow, and the direction of propagation of the ocean wave front. The angle of the person standing aboard the ship is defined as the counterclockwise rotation from the initial standing position where the person is facing the $x$ direction. These two angle conventions can be seen in Figure 74. The ship motions used corresponded to sea state 6 conditions with ship headings ranging from 0 degrees through 90 degrees in 15 degree increments. It was then assumed that a person with a foot length of $30 \mathrm{~cm}$ and standing with their feet $50 \mathrm{~cm}$ apart was on the deck. The simulation was run to find the $x$ and $y$ coordinates of the normal force. The location of the normal force was then plotted and can be seen in Figure 75 for a 


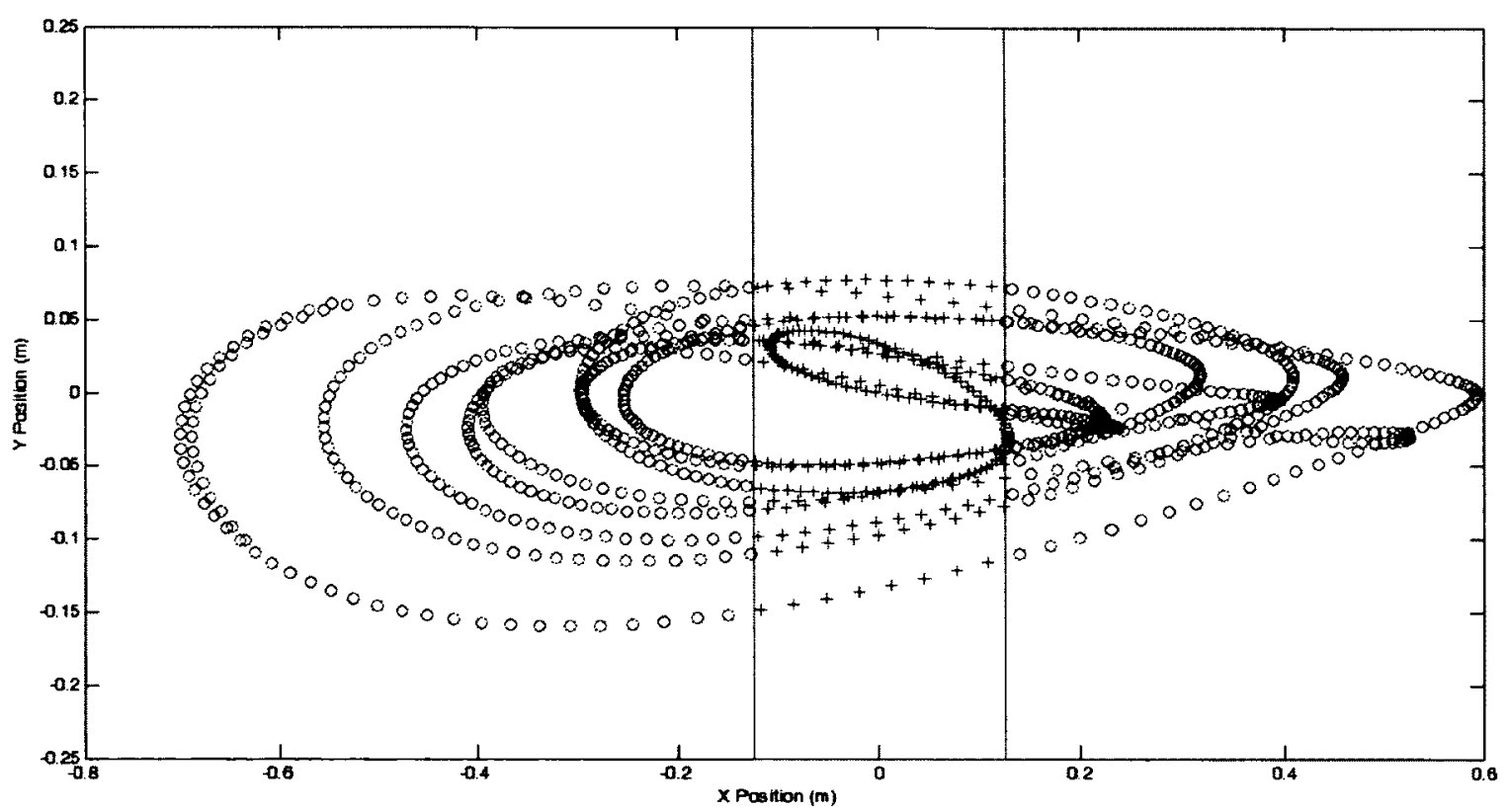

Figure 70: Coordinates of the normal force for $25 \mathrm{~cm}$ foot length footprint facing the $y$ direction.

heading of 30 degrees. The optimum angle was then found by changing the angle between the feet by advancing one foot in front of the other until the desired angle was obtained. The optimum footprint can also be seen in Figure 75 as the parallelogram outline. The simulation was run for all the cases and the optimum stance angle was found. The plot of the optimum stance angle versus the heading of the ship is shown in Figure 76. This plot shows that the optimum stance angle has a parabolic relation to the angle of the ship heading.

The size of the overall footprint can change the occurrence of MIIs for shipboard applications. Changing the length of the foot from a larger to a smaller size can increase the number of MIIs by a small number every 100 seconds. The width of the stance can significantly reduce the number of MIIs. This reduction in MII events all depends on the heading of the ship in relation to the principal wave direction. The increase in stance width would decrease the number of MIIs in the optimum stance 


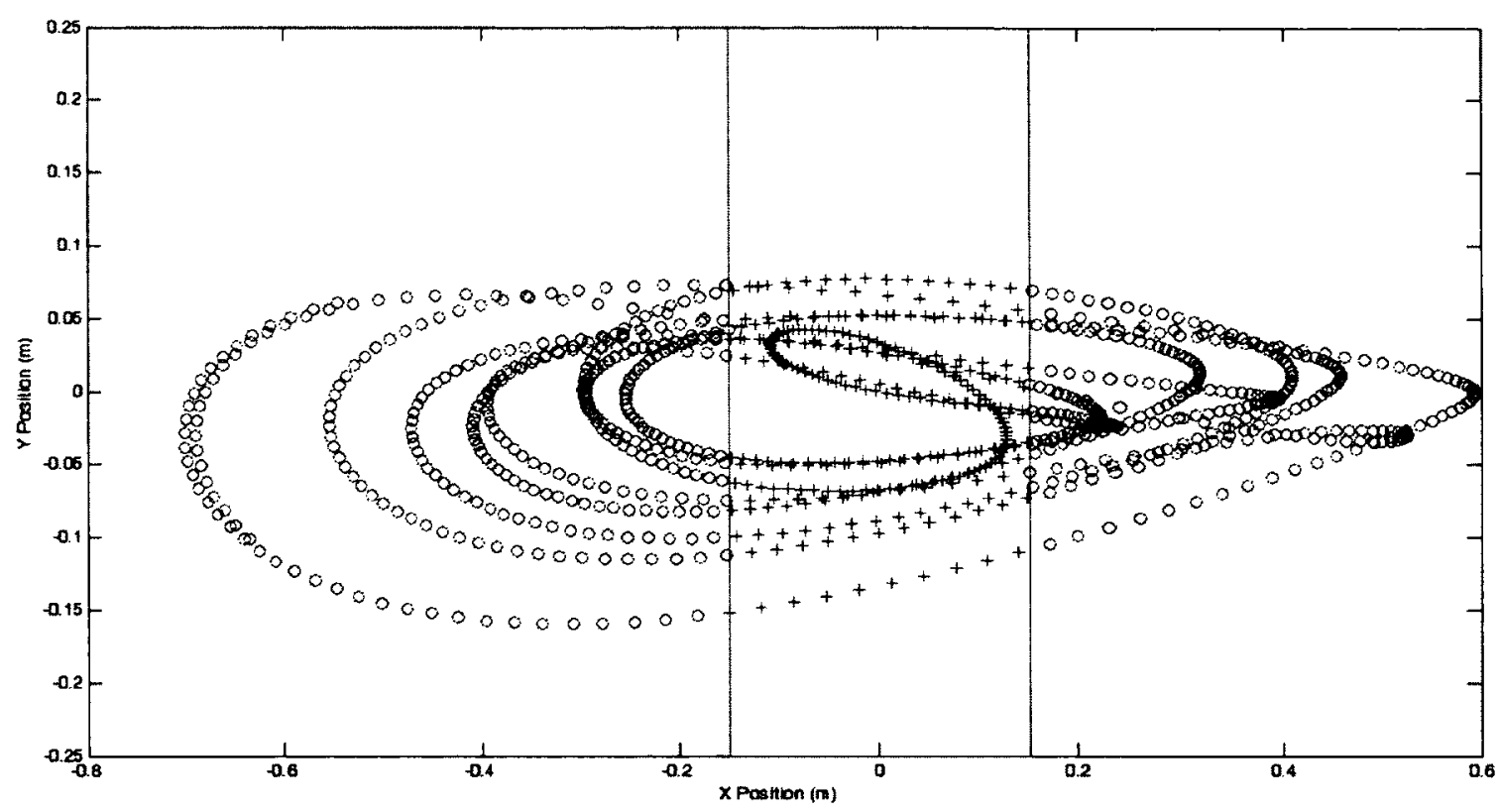

Figure 71: Coordinates of the normal force for $30 \mathrm{~cm}$ foot length footprint facing the $y$ direction.

direction. The optimum stance has been found to be related to the ship heading by a parabolic relation. The next section will investigate the effect of an unsteady load on the number of MII events. 


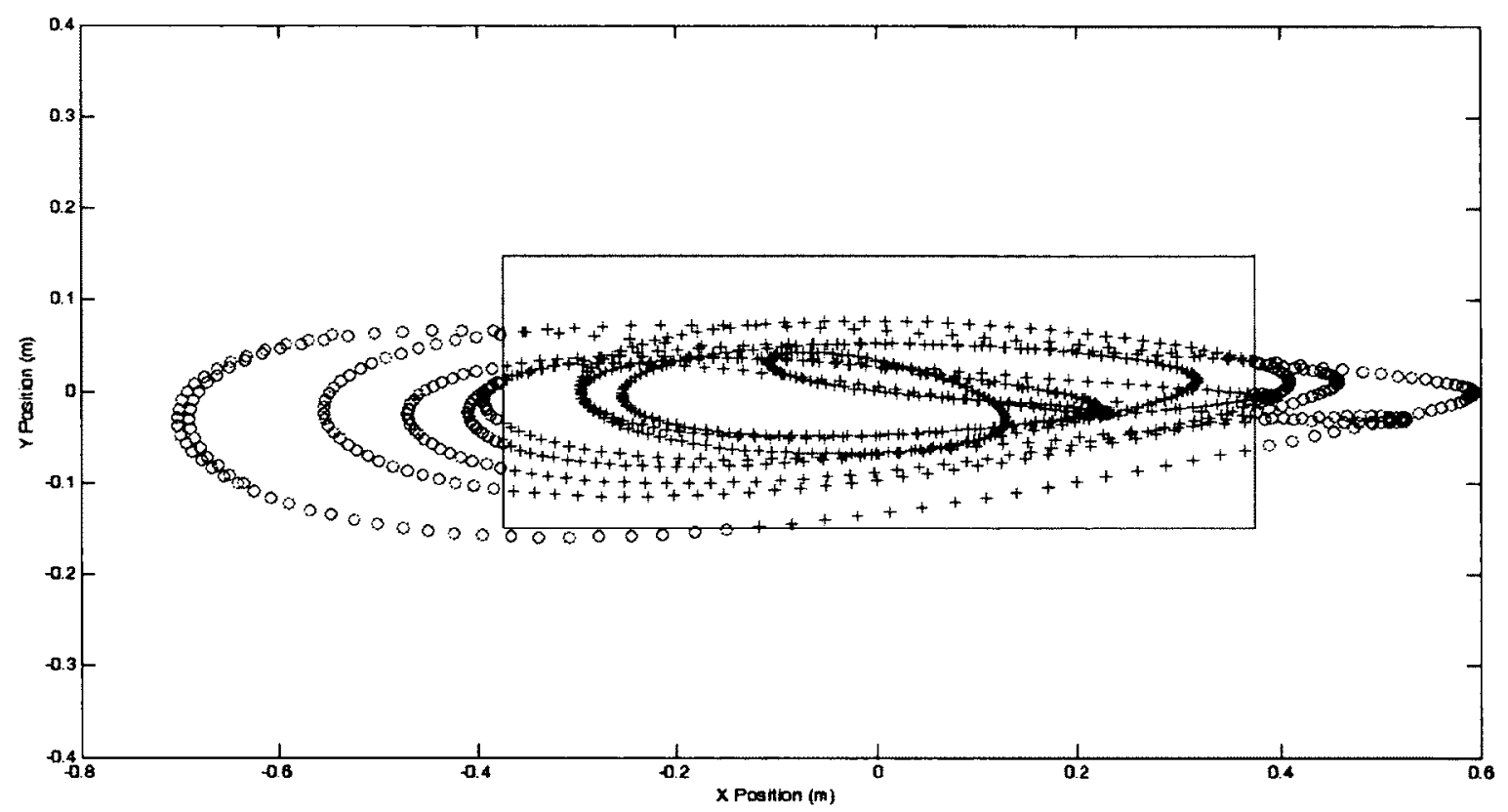

Figure 72: Coordinates of the normal force with a $75 \mathrm{~cm}$ wide straight stance.

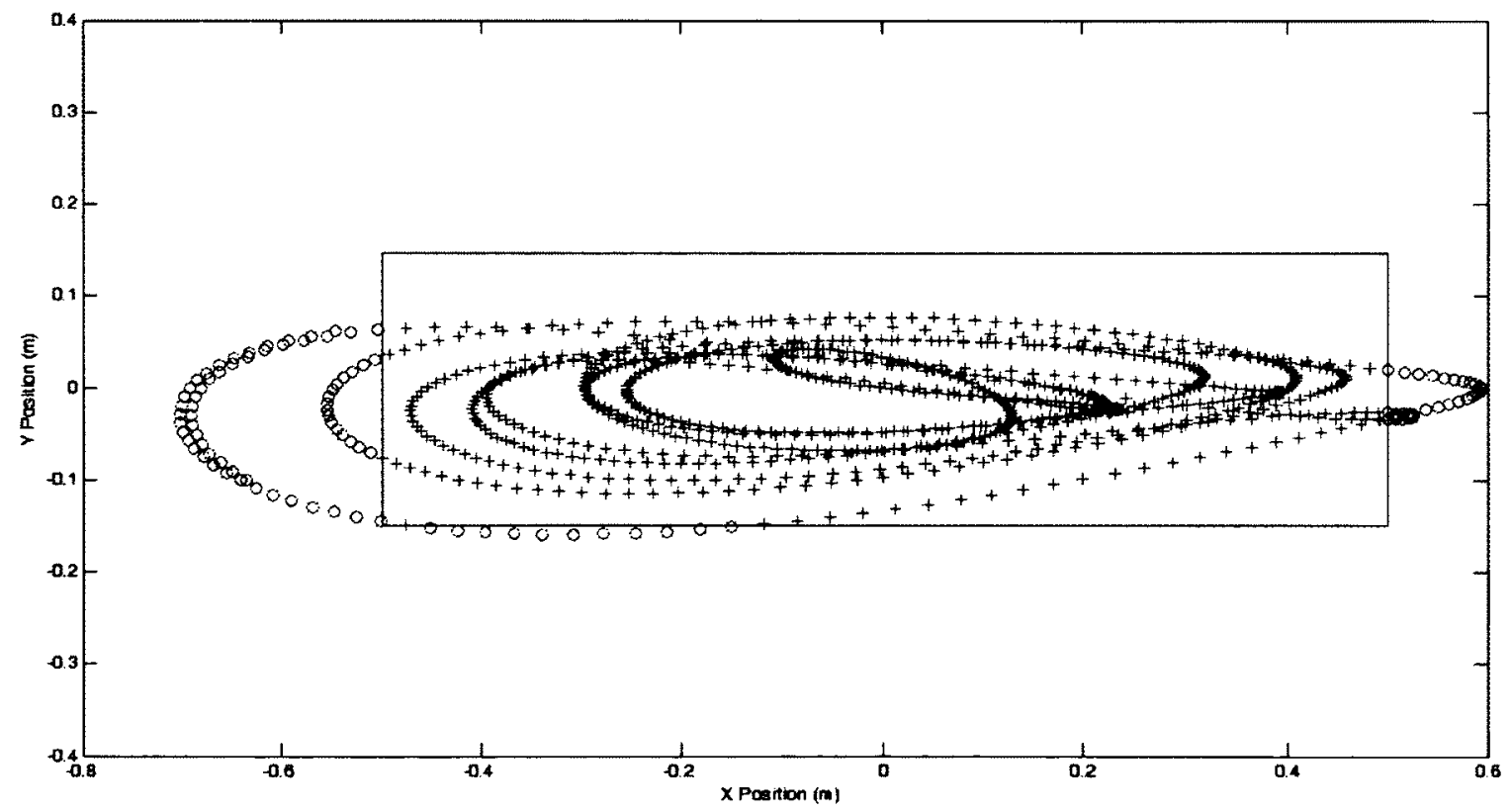

Figure 73: Coordinates of the normal force with a $100 \mathrm{~cm}$ wide straight stance. 


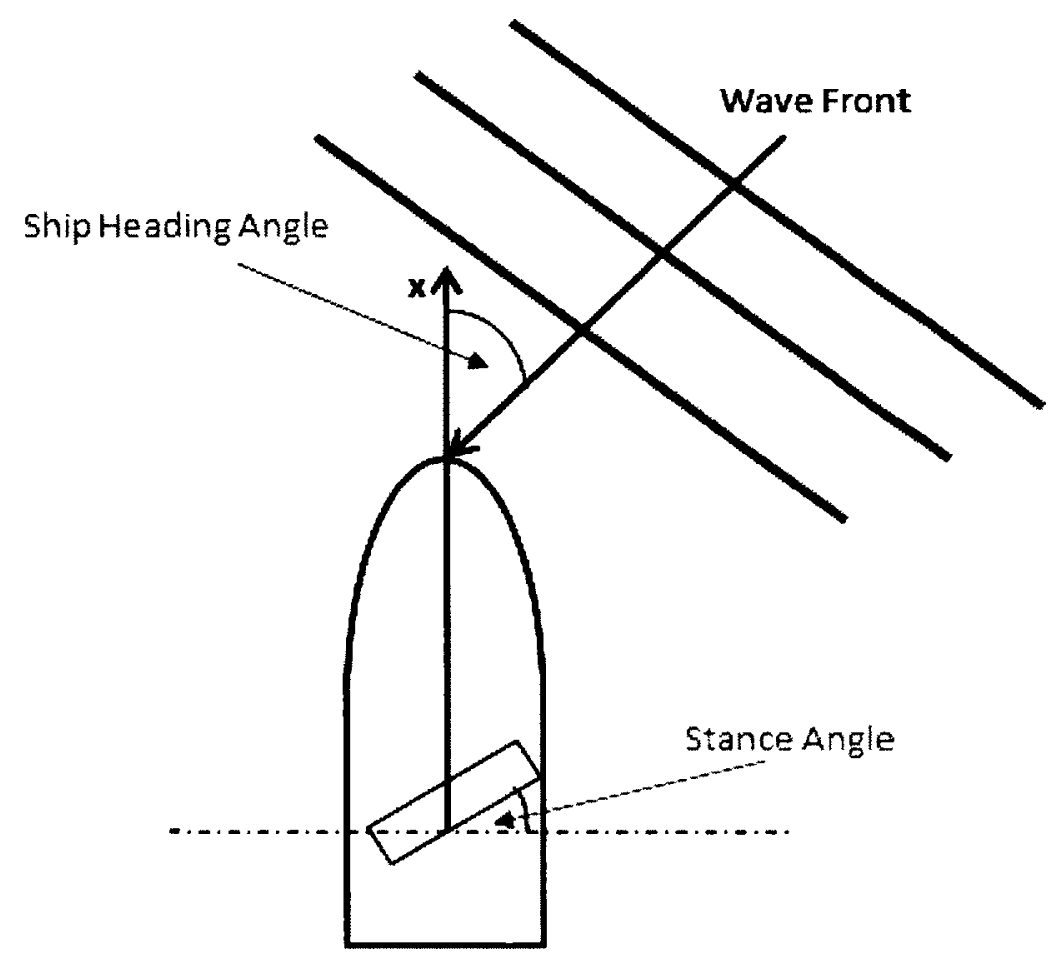

Figure 74: Diagram of angle conventions for the stance angle and ship heading angle. 


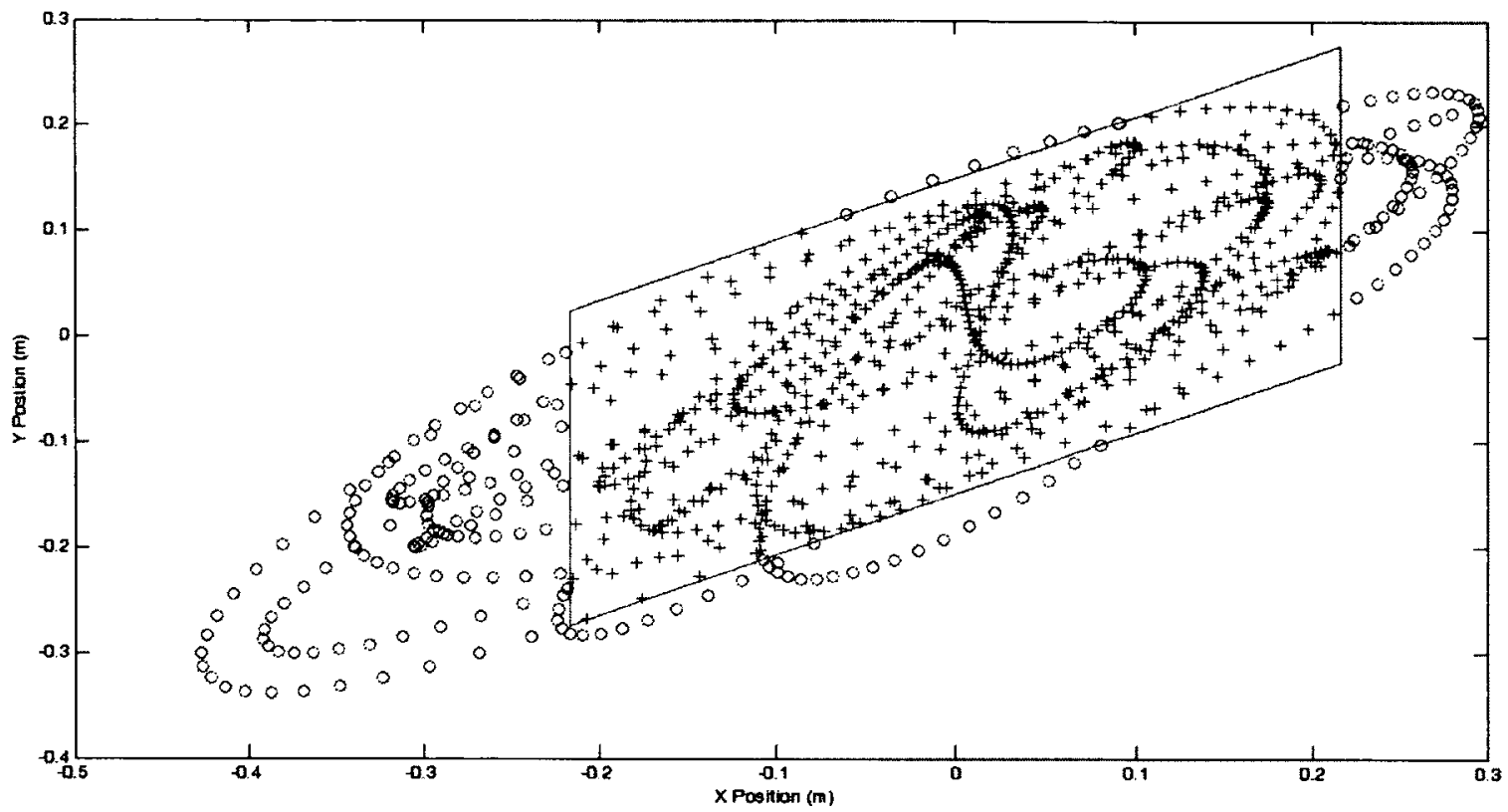

Figure 75: Optimum stance with a ship heading of 30 degrees.

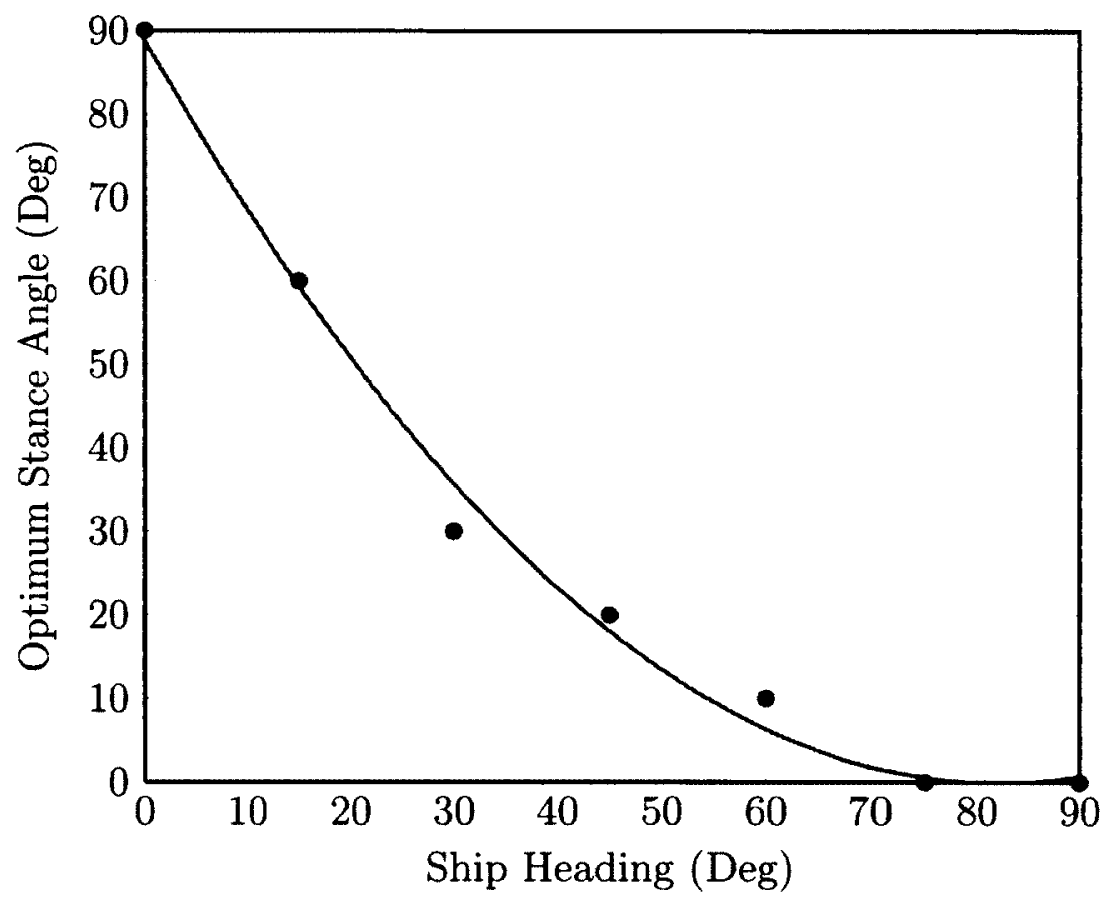

Figure 76: Trend of the optimum stance angle against ship heading relative to the wave direction. 


\subsection{Effect of Unsteady Dynamic Loads}

This section provides an investigation into the effect of unsteady loads on postural stability. The two load models used in this study are the pendulating load and the cart load models derived in Chapter 2. This investigation was focused at determining the extent of the effect that an external load on a person has on their overall postural stability, and which of the load types, if any, cause more MIIs over the other.

The main parameters that could affect postural stability are the mass of the load, the sea conditions, and the type of load, pendulating and cart in this case. Simulations were run varying these parameters in order to see the effect on the number of MIIs counted between each test. The parameters used for the simulated person were those for the physical Graham model presented in Chapter 3, and can be found in Table 11. The load models used in this investigation are the pendulum and cart load models as presented earlier. Typical cart masses and a pendulum of similar properties to those as the human model can be seen in Tables 16 and Table 17. Three masses were used for each of the load models. The simulations were run for sea states 4,5 , and 6 , with ship headings of 0,45 , and 90 degrees relative to the wave directions. Also, the interface force was limited to $500 \mathrm{~N}$ for this study.

A footprint of $50 \mathrm{~cm}$ wide by $25 \mathrm{~cm}$ long was chosen for this study. This represents a person with a median foot length with their feet held shoulder width apart. The cart was oriented such that the translation of the bottom mass was aligned with the $x$ direction of the ship. The results from the number of MIIs counted for each sea state can be seen in Tables 18 through 20 . The tables include the results for each mass with the specified heading.

The results show that the pendulating load causes an increased number of MII events over the case with no load attached for sea states 5 and 6 . The cart load results in the same number of MII events for the 0 and 45 degree headings for each mass and 
Table 16: Simulation parameters used for CRT3D load investigation

\begin{tabular}{cc}
\hline Parameter & Value \\
\hline$m_{1}$ & $50,70,90 \mathrm{~kg}$ \\
$m_{2}$ & $10,10,10 \mathrm{~kg}$ \\
$I_{x x 1}$ & $10000 \mathrm{~kg} \mathrm{~m}^{2}$ \\
$I_{y y 1}$ & $1000 \mathrm{~kg} \mathrm{~m}^{2}$ \\
$I_{z z 1}$ & $1000 \mathrm{~kg} \mathrm{~m}^{2}$ \\
$I_{x x 2}$ & $10000 \mathrm{~kg} \mathrm{~m}^{2}$ \\
$I_{y y 2}$ & $1000 \mathrm{~kg} \mathrm{~m}^{2}$ \\
$I_{z z 2}$ & $1000 \mathrm{~kg} \mathrm{~m}$ \\
$\mathbf{r}_{C G / A}^{M O}$ & $\{0.00 .00 .9\}^{T} \mathrm{~m}$ \\
$\mathrm{~g}$ & $9.81 \mathrm{~m} / \mathrm{sec}^{2}$ \\
\hline
\end{tabular}

across sea states. There are a reduced number of MII events for the 90 degree heading. This indicates that the cart causes fewer instabilities than the hanging pendulum. For visualization purposes the results from the sea state 6 case, at 45 degrees heading, with the $70 \mathrm{~kg}$ load have been graphed in order to see the movement of the normal force about the footprint geometry. The graphs can be seen in Figures 77 through 79 . It can be seen from Figure 78 that the normal force crosses the footprint boundary more often than the no load case. 
Table 17: Simulation parameters used for PND3D load investigation

\begin{tabular}{cc}
\hline Parameter & Value \\
\hline$m$ & $50,70,90 \mathrm{~kg}$ \\
$I_{x x}$ & $78555 \mathrm{~kg} \mathrm{~m}^{2}$ \\
$I_{y y}$ & $777746 \mathrm{~kg} \mathrm{~m}^{2}$ \\
$I_{z z}$ & $6060 \mathrm{~kg} \mathrm{~m}{ }^{2}$ \\
$\mathbf{r}_{C G / A}^{M O}$ & $\{-0.60 .340 .75\}^{T} \mathrm{~m}$ \\
$\mathrm{~g}$ & $9.81 \mathrm{~m} / \mathrm{sec}^{2}$ \\
\hline
\end{tabular}

Table 18: Simulation results for number of MIIs occurring at sea state 4

\begin{tabular}{clcc}
\hline & & Pendulum & Cart \\
\hline No Load & $@$ 0 degrees heading & 0 & 0 \\
& $@ 45$ degrees heading & 0 & 0 \\
& $@ 90$ degrees heading & 0 & 0 \\
$50 \mathrm{~kg}$ mass & $@ 0$ degrees heading & 0 & 0 \\
& $@$ @ 45 degrees heading & 0 & 0 \\
& $@ 90$ degrees heading & 0 & 0 \\
$70 \mathrm{~kg}$ mass & $@ 0$ degrees heading & 0 & 0 \\
& $@$ @ 45 degrees heading & 0 & 0 \\
& $@ 90$ degrees heading & 0 & 0 \\
$90 \mathrm{~kg}$ mass & $@ 0$ degrees heading & 0 & 0 \\
& $@ 45$ degrees heading & 0 & 0 \\
& $@ 90$ degrees heading & 0 & 0 \\
\hline
\end{tabular}


Table 19: Simulation results for number of MIIs occurring at sea state 5

\begin{tabular}{clcc}
\hline & & Pendulum & Cart \\
\hline \multirow{5}{*}{ No Load } & @ 0 degrees heading & 4 & 4 \\
& @ 45 degrees heading & 0 & 0 \\
& @ 90 degrees heading & 3 & 3 \\
$50 \mathrm{~kg}$ mass & @ 0 degrees heading & 13 & 4 \\
& @ 45 degrees heading & 5 & 0 \\
& @ 90 degrees heading & 5 & 2 \\
$70 \mathrm{~kg}$ mass & @ 0 degrees heading & 13 & 4 \\
& @ 45 degrees heading & 5 & 0 \\
& @ 90 degrees heading & 5 & 2 \\
& @ 0 degrees heading & 13 & 4 \\
& @ 45 degrees heading & 5 & 0 \\
& @ 90 degrees heading & 5 & 2 \\
\hline
\end{tabular}

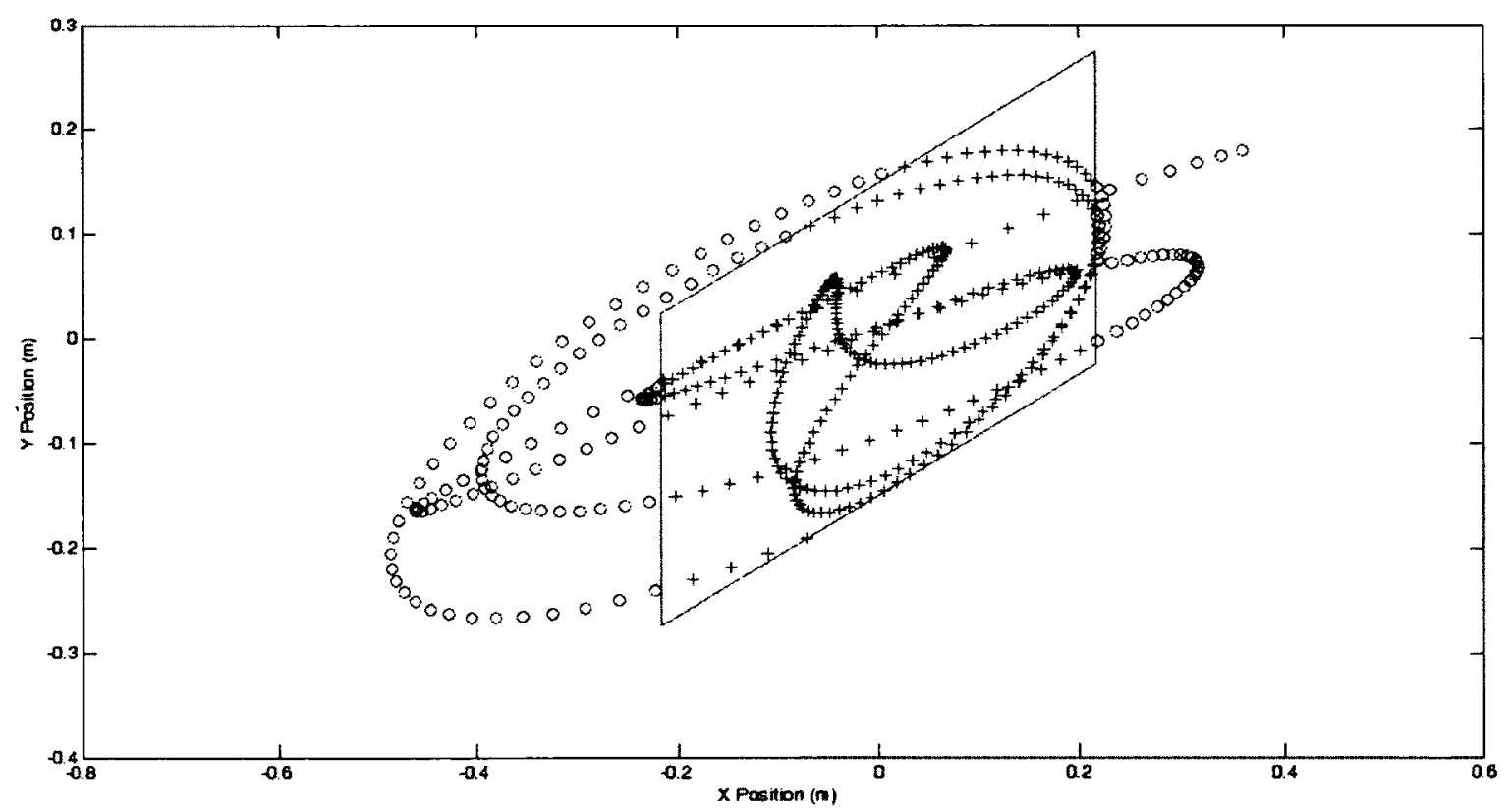

Figure 77: Coordinates of the normal force with no load attached. 
Table 20: Simulation results for number of MIIs occurring at sea state 6

\begin{tabular}{|c|c|c|c|}
\hline & & Pendulum & Cart \\
\hline \multirow[t]{3}{*}{ No Load } & $@ 0$ degrees heading & 24 & 24 \\
\hline & @ 45 degrees heading & 20 & 20 \\
\hline & @ 90 degrees heading & 12 & 12 \\
\hline \multirow[t]{3}{*}{$50 \mathrm{~kg}$ mass } & @ 0 degrees heading & 25 & 24 \\
\hline & @ 45 degrees heading & 22 & 21 \\
\hline & @ 90 degrees heading & 14 & 10 \\
\hline \multirow[t]{3}{*}{$70 \mathrm{~kg}$ mass } & @ 0 degrees heading & 25 & 24 \\
\hline & @ 45 degrees heading & 21 & 21 \\
\hline & @ 90 degrees heading & 14 & 10 \\
\hline \multirow[t]{3}{*}{$90 \mathrm{~kg}$ mass } & @ 0 degrees heading & 25 & 24 \\
\hline & @ 45 degrees heading & 22 & 21 \\
\hline & @ 90 degrees heading & 14 & 10 \\
\hline
\end{tabular}

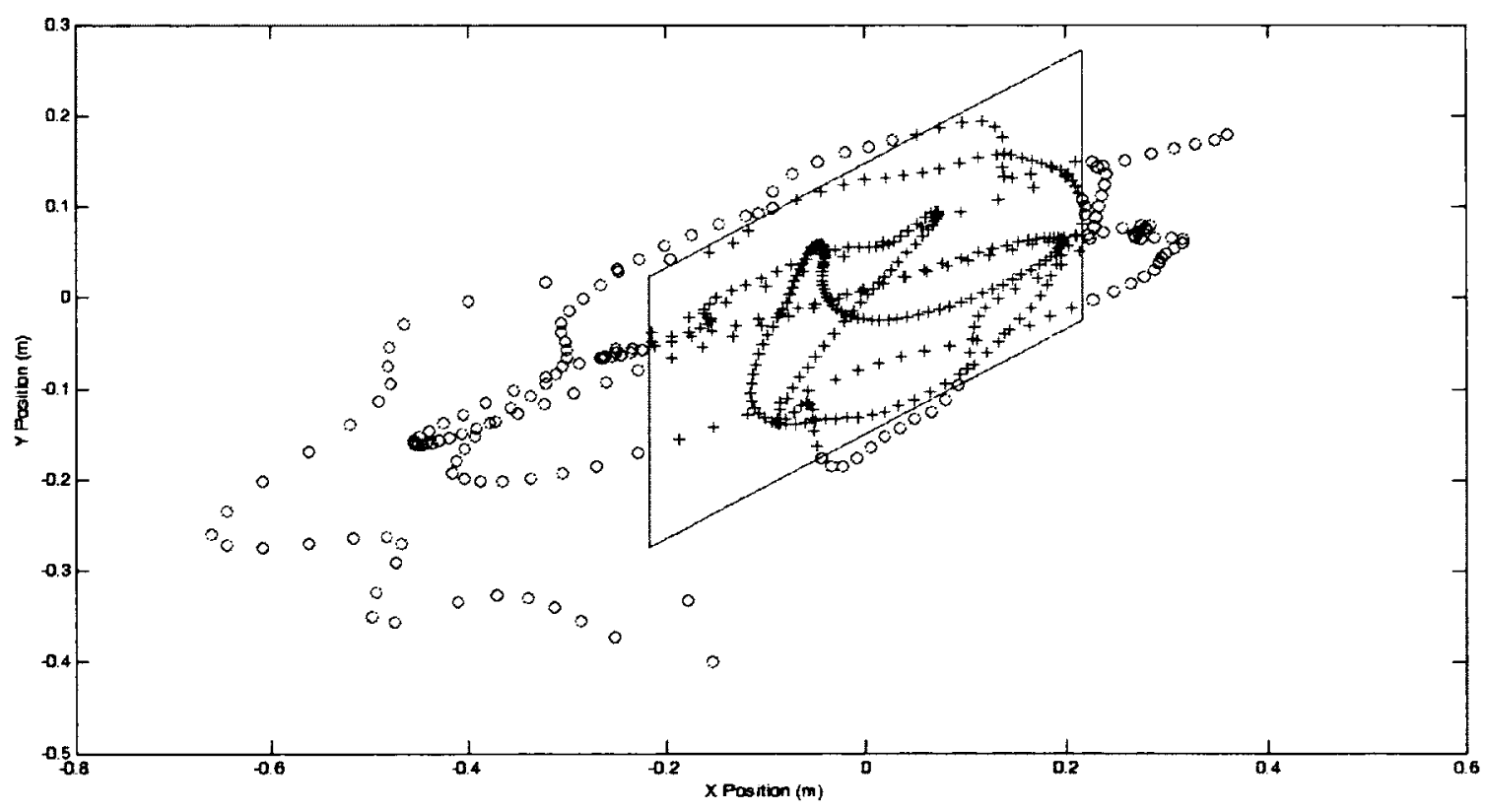

Figure 78: Coordinates of the normal force with the pendulum load attached. 


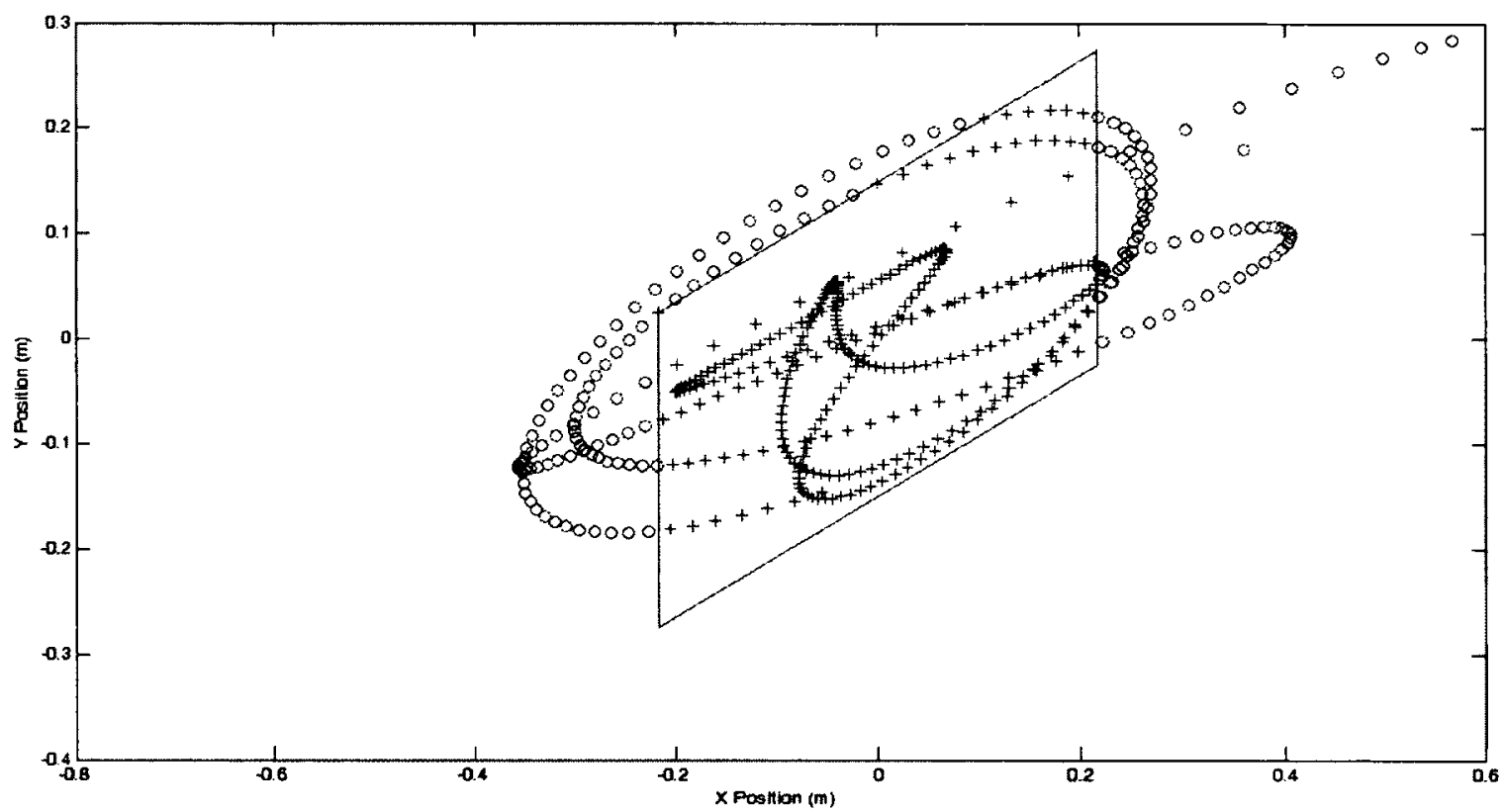

Figure 79: Coordinates of the normal force with the cart load attached. 


\section{Chapter 5}

\section{Conclusion}

\subsection{Discussion of Results}

This section provides a discussion of the results obtained from the validation experimentation and the simulation studies on the effects of stance geometry and external load. First the validation with the inverted pendulum will be discussed followed by the results from the harmonic simulations of the cart model, and then the results from the physical experimentation. Finally, the implications of the results from the simulation study on the stance model will be discussed.

The generalized Graham model was validated using two separate methods. The first was the comparison with the inverted pendulum model presented in Section 3.2 and the second was with results from physical experiments presented in Section 3.4. First the validation with the inverted pendulum will be discussed.

The result obtained from the Graham model and the Inverted pendulum follow the same trend and are of approximately the same magnitude. At the higher displacements of the ship motion the Graham model is showing higher forces and moments at the peaks. This can be explained by the difference in the calculations of the forces and moments between the two models. When the inverted pendulum is locked, for the calculations it is assumed to start at a zero degree angle relative to the deck. The 
forces and moments were then calculated using the same state equations as if the pendulum were not locked. Since the equations of motion are solving for the angular acceleration, it means for each time step a small angle will be calculated. After the forces and moments were calculated the pendulum was then simulated to be placed back at zero degree angles. This made the results appear as if the pendulum was not moving, where in each time step it was moving by a small angle. Since the pendulum was moving at a small angle, the forces and moments calculated from that model are expected to be calculated smaller than if the model was not moving. As well, for higher ship motion the angle is expected to be larger causing the calculated forces to be smaller, so the error between the Graham calculations and the inverted pendulum calculations is expected to get larger at the peaks of ship motion. The results from this study show that the Graham model is validated with the inverted pendulum model, and that the errors in the data are as expected.

The results from the validation of the Graham model through experimentation also validated the model. The results, as seen in Figures 42 through 47, show that the magnitude and trends of the data collected are similar. The simulation data does not appear to be as smooth as the collected data from the apparatus. The load cell results were filtered using a Butterworth filter that eliminated the high frequency noise similar to that seen in the simulation results. Another point is that the results from the moments calculated in the $y$ direction are not as similar as the results from the other directions. This error could have come from the calculations to rotate the experimentally-collected data to the model coordinate frame, but this error would not be isolated to one of the directions. It would be thought that it would cause an error in both the $x$ and $y$ directions. However, the error in the results is relatively small and thus the model is considered to be adequately validated through the experiments.

Validation of the cart was difficult considering that the cart by itself is not stable. It would have been dangerous to mount the cart to the platform and perform physical 
experiments by itself so another method had to be devised. Stabilizing the model with a spring and damper caused the model to be stable and therefore not a danger. The apparatus, however, was not fitted with the hardware to be able to attach a spring and damper system to the rails. There was not the option to attach them to the cart either. This limitation of the apparatus meant that the cart when operating uncoupled from the stability model had to be validated through theoretical frequency responses. This method validated that the model reacted as expected for a sinusoidal input. This method of validation is acceptable because if the dynamic model of the cart was not working correctly this would also be seen in the frequency analysis. In order for the frequency analysis to work, the velocity and acceleration of the cart had to be calculated correctly because it is directly related to the spring force and the damper resistance. If there was an error in calculating those forces then the error would have been seen in the frequency response plots.

The MII model with a generic footprint was also introduced in this thesis. The main idea behind the model came from the definition of an MII used by Graham [35]. The model was validated using the MII man physical apparatus to measure the number and time of the MII occurrences. The results from the validation experiments show that the simulation is able to determine the time and number of MII occurrences. There are a few potential sources of error in both the experimental and simulation MII counts. One of the major potential sources of error with the experimentation is that it was not safe to rebalance the apparatus in the lab when the platform was running. Some of the additional MIIs from the experiments could be caused by the inability to upright the apparatus while a run was being conducted. On Quest, the apparatus was balanced upright after each MII and the number and times of the MIIs are more consistent with the simulated data. Another point of interest with the results is that there is a delay in the time of the MII events counted from the experiment. This error is assumed to result from how the time is recorded with the 
Arduino. The timing of the results from the Arduino was based on counting milliseconds since the last communication. This function is also not connected to the computer clock and thus the timing might be different on board the Arduino. The delay could also be the result of the physical model having to accelerate over time before it could move enough to trigger the switch. The effect of the acceleration from the simulation would be instantaneous and would not have the delay. The validation of the generic stance model was completed by performing the experiments on a ship at sea. The data recorded from the experiments and the results from the simulation were similar.

Since the stance geometry model is validated, it can be used to determine some relationships regarding the effect of stance geometry and the effect of unsteady loads. First, the effect of stance geometry experiments developed a relationship between the relative heading of the ship and the optimum stance angle to minimize the number of MII events. This relationship was found to be parabolic. When a wave is coming to hit the ship at an oblique angle, the ship will pitch and roll. The combination of the pitch and roll will cause the deck to be at a combined angle between the roll and pitch. Combining the two angles will cause a parabolic relationship between the heading angle and the optimum orientation to stand for that given deck angle.

The effect of an unsteady load on postural stability was also investigated. The simulation cases were designed in order to find the relationship between the effects of the load in certain sea state, heading angles, and load weight. From the results it can be seen that the pendulating load causes an increased number of MIIs over not having any load. The cart however does not have an effect on the number of MII occurrences over no load. In some cases the cart load reduces the number of MII events. The change of the mass of the load used in these experiments did not show any relationship to the number of MII occurrences. This may be due to the possibility that the mass was not large enough to increase the number of MII events that were 
being cause with the smaller mass. The type of results that can be generated with the models, however, is key to knowing safe procedures for on deck operation.

\subsection{Conclusion}

The main purpose of this project was to develop a postural stability model the can be effected by an external unsteady load and investigate the effect of the unsteady load on the occurrence of MIIs. A three-dimensional Graham-based model was developed as the postural stability model and was validated through experiments with a physical apparatus. The load models used were a pendulating load developed by Langlois and a cart load model. The cart load model was validated using the frequency response because the model was unstable and could not be validated physically. The coupled models were validated using the physical apparatus.

The MII model developed for a generic stance footprint was validated using a physical model constructed with microswitches at the base to count the MII occurrences. The occurrences count from the experimentation matched that of the simulation with a minor delay caused by the response time of the apparatus. The MII experimental apparatus was also taken on board CFAV Quest for a heavy weather sea trial. The collected trial data also validated the stance model. The optimum angle to stand on deck in relation to the principal wave heading was found to be a parabolic relationship. It was also found that the pendulating load effects postural stability more than the cart. The model attached to the pendulating load had more MII events that the model attached to the cart. 


\subsection{Future Work}

One of the ways in which this project can be extended is with experimentation with humans interacting with the pendulum load model apparatus. The apparatus was designed to be a 1:1 scale with humans so that the postural stability model could be replaced by a human. There would have to be safety measures in place to ensure that the subject does not get injured during the process. As well, an ethics application would have to be submitted and approved prior to performing any experiments with human subjects. From these experiments the postural stability MII models could be tuned for different experience levels. It has been observed that a person that has been on board many ships will not have as many MII events as someone who has never been on a ship before. The data from experiments from human subjects could tune the simulations to be more like human behaviour as opposed to rigid structure behaviour.

As well, human testing can be performed in order to tune the stance geometry footprint MII model. From human experiments the subject would just have to perform quiet standing with predetermined stances. This would be a minimal risk for someone who is on board a ship regularly, as that is what they are doing all the time while on board. The model could be tuned in order to better predict human behaviour and stances that are used.

From a simulation perspective, the models could be enhanced in order to have a footprint that changes over time. In order for this to be accomplished the determination of whether the normal force is within the footprint needs to be embedded into the computational model. Currently that is done through post processing of the data. If the motion over time of the footprint is known, then at each time step it can be determined if the normal force is within the footprint or not, and thus know whether an MII has occurred. This could be used for models that would count the 
MII occurrences of a person walking on deck. All that would need to be known is how the footprint changes over time and the position on deck over time. From those the number of MIIs could be counted. This means that the simulated model would not have to remain fixed to the deck and can be moving and changing over time. 


\section{List of References}

[1] K. W. Tsui. Development of the Virtual Flight Deck - Real Time Simulation Environment. PhD thesis, Carleton University, Carleton University, 2009.

[2] H.P.M. Peeters, H.B. Caberg, and J.M.F. Mol. Evaluation of biomechanical models in posturography, medical and biological engineering and computing. Medical and Biological Engineering and Computing, 23(5):469-473, 1985. exp model ip.

[3] F. Benvenuti. Physiology of human balance. Advances in Neurology, 87:41-51, 2001.

[4] L.M. Nashner. Adaptation of human movement to altered environments. Trends in Neurosciences, 5:358-361, 1982.

[5] D.A. Winter, A.E. Patla, P. Francois, M. Ishac, and K. Gielo-Perczak. Stiffness control of balance in quiet standing. Journal of Neurophysiology, 5:1211-1221, 1998.

[6] K. Bothner and J. Jensen. How do non-muscular torques contribute to the kinetics of postural recovery following a support surface translation. Journal of Biomechanics, 34(2):245-250, 2001.

[7] F. Gubina, H. Hemami, and R. B. McGhee. On the dynamic stability of bipe locomotion. IEEE Transactions on Biomedical Engineering, 21(2):102-108, 1974.

[8] C. K. Chow and D. H. Jacobson. Further studies of human locomotion postural stability and control. Mathematical Biosciences, 15:93-108, 1972.

[9] M.P. Murray, A.A. Seireg, and S.B. Sepic. Normal postural stability and steadiness: Quantitative assessment. Journal of Bone and Joint Surgery, 57:510-516, 1975. 
[10] H. Hemami and C.L.J. Golliday. Postural stability of the two-degree-of-freedom biped by general linear feedback. IEEE Transations on Automatic Control, AC21:74-79, 1976.

[11] H. Hemami. The inverted pendulum and biped stability. Mathematical Biosciences, 34(2):95-110, 1977.

[12] H. Hemami and V.C. Jaswa. On a three-link model of the dynamics of standing up and sitting down. IEEE Transactions on Systems, Man and Cybernetics, SMC-8:120-135, 1978.

[13] H. Hemami, F.C. Weimer, C.S. Robinson, C.W. Stockwell, and S. Cvetkovic. Biped stability considerations with vestibular models. IEEE Trans. Automat. Contr., AC-23:1074-1079, 1978.

[14] L. M. Nashner. A model describing vestibular detectionof body sway motion. Acta Otolaryngol, 72:429-436.

[15] S.H. Koozekanani, C.W. Stockwell, R.B. McGhee, and F. Firoozmand. On the role of dynamic models in quantitative posturography. IEEE Transactions on Biomedical Engineering, BME-27(10):605-609, 1980.

[16] C.W. Stockwell, S.H. Koozekanai, and K. Barin. A physical model of human postural dynamics. Annals of the New York Academy of Sciences, 374:722-730, 1981.

[17] H. Hemami, M.J. Hines, R.E. Goddard, and B. Friedman. Biped sway in the frontal plane with locked knees. IEEE Transactions on Systems, Man and Cybernetics, SMC-12(4):577-582, July/August 1982.

[18] H. Hemami and B. Chen. Stability analysis and input design of a two-link planar biped. The International Journal of Robotics Resesarch, 3(2):93-100, 1984.

[19] K. Barin. Evaluation of a generalized model of human postural dynamics and control in the sagittal plane. Journal of Biological Cybernetics, 61:37-50, 1989.

[20] G. E. Riccio, E. J. Martin, and T. A. Stoffregen. The role of balance dynamics in the active perception of orientation. Journal of Experimental Psycology Human Perception and Performance, 18(3):624-644, 1992.

[21] G. E. Riccio and T. A. Stoffregen. Gravitoinertial force versus the direction of balance in the perception and control of orientation. Psychological Review, 97(1):135-137, 1990. 
[22] G. E. Riccio. Information in movement variability about the qualitative dynamics of posture and orientation. Variability and Motor Control, pages 317-357, 1993.

[23] K. Iqbal, H. Hemami, and S. Simon. Stability and control of a frontal four-link biped system. IEEE Transations on Biomedical Engineering, 40(10):1007-1018, 1993.

[24] J.L. Patton, Y.C. Pai, and W.A. Lee. A simple model of the feasible limits to postural stability. In 19th International IEEE Conference, Chicago, IL, USA, 1997.

[25] J. L. Patton, Y. C. Pai, and W. A. Lee. Evaluation of a model that determines the stability limits of dynamic balance. Gaite and Posture, 9:38-49, 1999.

[26] S. M. Slobounov, S. A. Moss, E. S. Slobounova, and K. M. Newell. Aging and time to instability in posture. Journal of Gerontology; Biological Sciences, 53A(1):B71-B78, 1998.

[27] Q. Wu, A. B. Thornton-Trump, and N. Sepehri. Lyapunov stability control of inverted pendulums with general base point motion. International Journal of Non-Linear Mechanics, 33(5):801-818, 1998.

[28] Q. Wu and R. Swain. On lyapunov's stability control of two-link base excited inverted pendulums with applications to human locomotion. In IEEE International Conference on Systems, Man, and Cybernetics, 2000.

[29] H. Hemami, K. Barin, and Y. Pai. Quantitative analysis of the ankle strategy under translational platform disturbances. IEEE Transactions on Neural Systems and Rehabilitation Engineering, 14:4:470-480, 2006.

[30] M. Schmid and S. Conforto. Stability limits in the assessment of postural control through the time-to-boundary function. 2007.

[31] L. Humphrey, H. Hemami, K. Barin, and A. Krishnamurthy. Simulated responses to support surface disturbances in humanoid biped model with a vestibular-like apparatus. IEEE Transactions on Systems, Man and Cybernetics, 40(1):109-119, 2010.

[32] J. Matthews, S. Mackinnon, W. Albert, M. Holmes, and A. Patterson. Effects of moving environments on the physical demands of heavy materials handling operators. International Journal of Industrial Ergonomics, 37(1):43-50, 2007. 
[33] A.E. Baitis, T.R. Applebee, and T.M. McNamara. Human factors considerations applied to operations of the FFG-8 and LAMPS MK III. Naval Engineers Journal, 97(4):191-199, 1984.

[34] R. Graham, A.E. Baitis, and W.G. Meyers. On the development of seakeeping criteria. Naval Engineers Journal, 104:259-275, 1992.

[35] R. Graham. Motion induced interruptions as ship operability criteria. Naval Engineers Journal, 102:65-71, 1990.

[36] B.E. Maki, W.E. Mcllroy, and S.D. Perry. Influence of lateral destabilization on compensatory stepping responses. Journal of Biomechanics, 29(3):343-353, 1996.

[37] P. Crossland. Using the large motion simulator (LMS) at DRA bedford to investigate the effects of ship motions on motion induced interruptions. Technical Report DRA/SS/SSHE/CR96008, Defence Research Agency, February 1996.

[38] P. Crossland, K.J.N.C. Rich, and D. Granshaw. Validating a model for predicting motion induced interruptions to task performance using simulated motions from the FFG-8 and Type 23 frigate. Technical Report DERA/SS/HE/CR971017, Defence Evaluation and Research Agency, 1997.

[39] P. Crossland and K. Rich. Validating a model of the effects of ship motion on postural stability. International Conference of Environmental Ergonomics, pages 385-388, 1998.

[40] W.E. Mcllroy and B.E. Maki. Task constraints on foot movement and the incidence of compensatory stepping following pertubation of upright stance. Brain Research, 616:30-38, 1993.

[41] B.E. Maki and W.E. McIlroy. The role of limb movements in maintaining upright sance: The "change-in-support" strategy. Physical Therapy, 77(5):488-507, 1997.

[42] J. McKee. Simulating the Effects of Ship Motion on Postural Stability Using Articulated Dynamic Models. PhD thesis, Carleton University, Carleton University, 2004.

[43] A.E. Baitis, F.D. Holcomb, S.L. Conwell, P. Crossland, J.H. Patton, and R. Strong. Motion induced interruption (MII) and motion induced fatigue (MIF) experiments at the naval biodynamics laboratory. Technical Report CRDKNSWE-HD-1423-01, Carderock Division, Naval Surface Warfare Center, 1995. 
[44] R.G. Langlois. Development of a spatial inverted pendulum shipboard postural stability model. In O. Turan, J. Bos, J. Stark, and J.L. Colwell, editors, International Conference on Human Performance at Sea Proceedings, pages 137-148, Glasgow, 2010.

[45] C. A. Duncan, S. N. MacKinnon, W. J. Alberta, D. M. Antlec, and J. Matthews. Effect of simulated vessel motions on thoracolumbar and centre of pressure kinematics. Occupational Ergonomics, 7:265-274, 2007.

[46] C.A. Duncan, S.N. MacKinnon, and W.J. Albert. Changes in thoracolumbar kinematics and centre of pressure when performing stationary tasks in moving environments. Technical report, Memorial University of Newfoundland, July 2009 .

[47] C.J. Hasson, R.E.A. Van Emmerik, and G.E. Caldwell. Predicting dynamic postural instability using center of mass time-to-contact information. Journal of Biomechanics, pages 2121-2129, 2008.

[48] A.L. Hof, M.G.J. Gazendam, and W.E. Sinke. The condition for dynamic stability. Journal of Biodynamics, 38:1-8, 2005.

[49] Military Agency for Standardization, NATO. Standardized wave and wind environments and shipboard reporting of sea conditions. STANAG No. 4194, 1983. 